\title{
Roles of Akt signaling and its downstream pathways in wood frog freeze tolerance
}

\author{
Jing Zhang \\ B.Sc. Suzhou University of Science and Technology \\ (China, People's Republic of)
}

A Thesis Submitted to the Faculty of Graduate Studies and Research in partial fulfillment of the requirements of the degree of

Doctor of Philosophy
Department of Biology

Carleton University

Ottawa, Ontario, Canada 


\title{
Acceptance sheet
}

The undersigned hereby recommend to the Faculty of Graduate Studies and Research acceptance of this thesis

\section{Roles of Akt signaling and its downstream pathways in wood frog freeze tolerance}

\author{
submitted by \\ Jing Zhang, B.Sc. \\ in partial fulfillment of the requirements for the degree of Doctor of Philosophy
}

Chair, Department of Biology

Thesis Supervisor

External Examiner

Carleton University 


\section{ABSTRACT}

Wood frogs, Rana sylvatica, are one of only a few vertebrate species that survive prolonged whole body freezing during the winter. Multiple adaptations of physiology and biochemistry that support freeze tolerance have been identified including accumulation of extreme levels of glucose as a cryoprotectant and entry into a hypometabolic state that reduces the energy needs of the animal while frozen. To date, the stress responsive signal transduction networks that trigger and regulate these adaptations have received little attention. The current thesis addressed this subject by exploring responses and regulation of a major intracellular signaling pathway (the Akt pathway) that is centrally involved in mediating cellular growth and proliferation responses, typically responding to extracellular insulin signals. Analysis of four organs (liver, kidney, heart and skeletal muscle) showed activation of the Akt pathway in liver but signs of inhibition occurred in other tissues in response to freezing. Activation of Akt-dependent anti-apoptosis mechanisms in liver was also indicated to support cell survival in the frozen, anoxic state. However, analysis of multiple protein components of the cell cycle and TORC1dependent protein synthesis showed strong suppression of these in all tissues, although with lesser inhibition in liver. This demonstrates the importance of suppressing energyexpensive cell processes under stress conditions. The data show that, during whole body freezing of wood frogs, (1) ATP expensive cellular events such as the cell cycle and protein synthesis were suppressed; (2) liver remains more metabolically active than other tissues tested; and (3) freeze responsive Akt activation in liver does not universally activate all of its downstream pathways but rather selectively triggers specific targets, particularly those important to glucose production as a cryoprotectant and to cell 
preservation. The thesis also investigated freeze-responsive antioxidant defenses in wood frog liver and muscle with a focus on mechanisms regulated by the Nrf2 transcription factor and showed that Nrf2 is glucose-responsive. Furthermore, glucose appears capable of differentially affecting gene expression and posttranslational modifications of proteins in liver. Overall, this thesis showed the central importance of the Akt pathway in freeze tolerance and demonstrated tissue- and environment-specific responses by the pathway and its downstream processes. 


\section{ACKNOWLEDGEMENTS}

First and foremost, I would like to thank my thesis supervisor Dr. Kenneth Storey. Dr. Storey introduced me to this amazing world of scientific research five years ago and continuously inspired me with his vision and philosophy throughout my doctoral study. My sincerest gratitude also goes to Jan Storey, for not only her excellent editing of my manuscripts, but also the invaluable advice and discussions of research life and beyond.

Special thanks to the members of my thesis committee, Dr. Shelley Hepworth and Dr. Steve Brooks, for their support and helpful advices.

I am forever grateful to the members of the Storey lab, past and present, who made the best five years of my life. My mentors: Oscar Aguilar, Craig Brooks, Dr. Ben Lant and Dr. Anastasia Krivoruchko; my colleagues: Shannon Tessier, Mike (Chen-Wei) Wu, Kyle Biggar, Neal Dawson, Ryan Bell, Katrina Sullivan, Alyx Holden, Yulia Maistrovski, Bryan Luu, Drew Rouble, Johny Abboud and Dr. Amal Malik; the promising new members: Sanoji Wijenayake, Christie Childers, Kama Szer and Dr. Barb Katzenback; and the latest generation whom I just had the pleasure of sharing the joy of scientific search with: Sam Logan, Saumya Bansal, Hanane Hadj-Moussa and Victoria Gerber.

Most importantly, I would like to thank my father Jian-Zhong Zhang, for his guidance in my younger years and many years to come, and also my mother Rong-Fen Zhou, who showed nothing but love and support.

Last but certainly not least, I would like to thank the animal model of this thesis and most of my doctoral research projects, the wood frog (Rana sylvatica). My research would not be possible without them and their exciting freezing tolerant strategies. 


\section{TABLE OF CONTENTS}

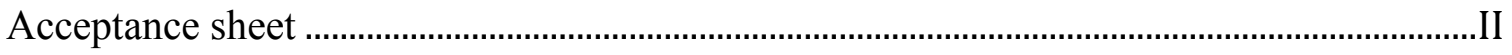

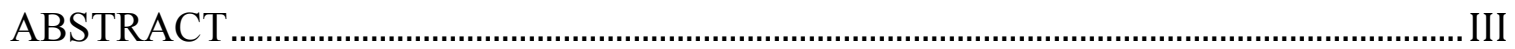

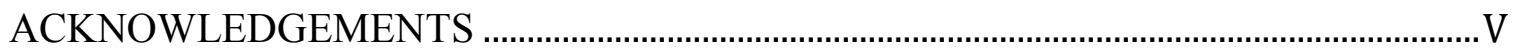

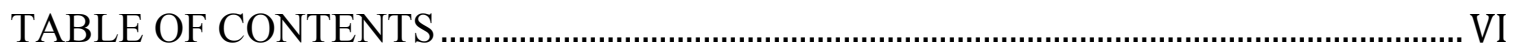

List of Abbreviations ...........................................................................................................

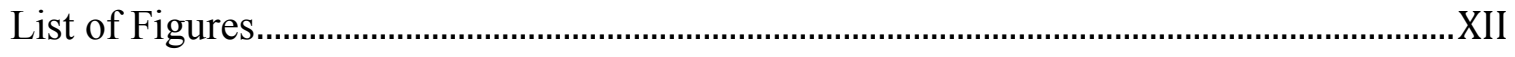

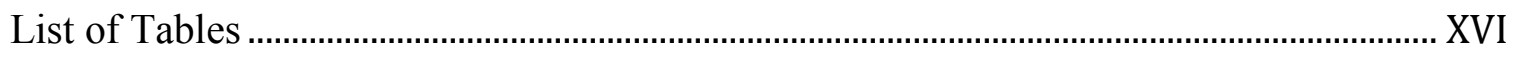

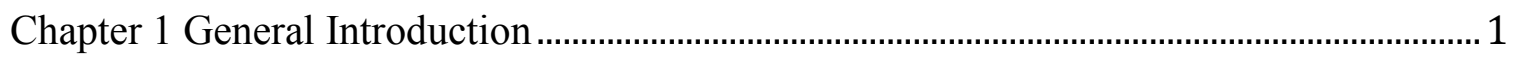

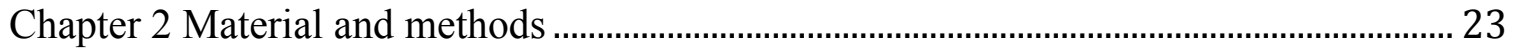

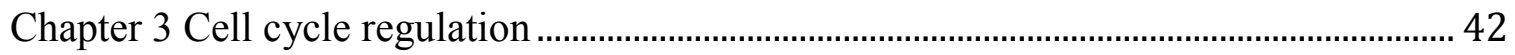

Chapter 4 Regulation and response of Akt signaling ............................................................. 77

Chapter 5 Regulation and response of the TORC1 pathway ................................................109

Chapter 6 Nrf2-dependent antioxidant defense ....................................................................144

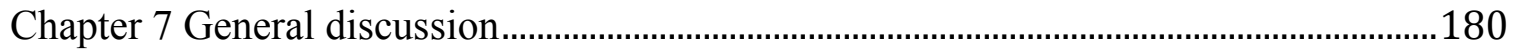

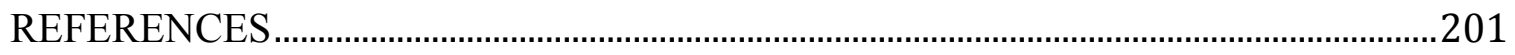

Appendix A: Explaination of immublotting ……...........................................................218

Appendix B: Communications at scientific meetings ..........................................................219 


\section{List of Abbreviations}

4EBP

AMPK

APS

ARE

ASK1

ATF

ATP

cAMP

Cdc

Cdk

CdkI

Chk

ChRE

Cip/Kip

CO-IP

$\mathrm{Cu} / \mathrm{Zn} \mathrm{SOD}$

DEPC

dNTP

DTT

E2F

EDTA

ELISA
eIF4E binding protein

AMP-activated protein kinase

Ammonium persulphate

Antioxidant responsive element

Apoptosis-signal-regulating kinase 1

Activating transcription factor

Adenosine triphosphate

cyclic AMP, or cyclic Adenosine monophosphate

cell division control protein

Cyclin-dependent kinase

Cdk inhibitor

Checkpoint kinase

Carbohydrate response element

CDK interacting protein/Kinase inhibitory protein

Co-Immunoprecipitation

Copper/zinc dependent superoxide dismutase

Diethylpyrocarbonate

Deoxynucleotide triphosphate

Dithiothreitol

E2 promoter binding factor

Ethylenediaminetetraacetic acid

Enzyme-linked immunosorbent assay 


\begin{tabular}{ll} 
EMSA & Electrophoretic Mobility Shift Assay \\
ENCODE & Encyclopedia Of DNA Elements \\
eIF & Eukaryotic initiation factor \\
cGMP & cyclic GMP, or cyclic Guanosine monophosphate \\
CK2 & Casein kinase \\
FOXO1 & Forkhead box protein O1 \\
G6P & Glucose-6-phosphate \\
G6PC & G6PC glucose-6-phosphatase, catalytic subunit \\
G6PDH & Glucose-6-phosphate dehydrogenase \\
GßL & G protein $\beta$-subunit-like protein \\
GAP & GTPase-activating protein \\
GDP & Guanosine diphosphate \\
GLUT & Glucose transporter \\
GP & Glycogen phosphorylase \\
GR & Glutathione reductase \\
GRE & Glucose response element \\
GRP & Glucose-regulated protein \\
GS & Glycogen synthase \\
GSH & Glutathione \\
GSK3 & Glycogen synthase kinase 3 \\
GST & Glutathione S-transferase \\
GTP & Guanosine triphosphate \\
GTP-RHEB & GTP bound Ras homologue enriched in brain \\
HDAC & Histone deacetylase \\
\hline
\end{tabular}


HEPES 4-(2-hydroxyethyl)-1-piperazineethanesulfonic acid

HRP Horseradish peroxidase

HSP Heat shock protein

IgG Immunoglobulin $\mathrm{G}$

INA Ice nucleating agents

INK4 Inhibitor of cyclin-dependent kinase 4

Keap1 Kelch-like ECH-associated protein 1

LC-MS Liquid chromatography - mass spectrometry

MAPK Mitogen-activated protein kinases

MEF2 Myocyte enhancer factor-2

miRISC miRNA-induced silencing complex

miRNA MicroRNA

mLST8 mammalian lethal with SEC13 protein 8

MnSOD Manganese dependent superoxide dismutase

MPF M-phase Promoting Factor

mRNA Messenger RNA

MRD Metabolic rate depression

NADPH Reduced form of $\mathrm{NADP}^{+}$, or nicotinamide adenine dinucleotide

phosphate

NP-40 Nonyl phenoxypolyethoxylethanol

Nrf2 Nuclear factor (erythroid-derived 2)-like 2

PAGE Polyacrylamide gel electrophoresis

PBS Phosphate-buffered saline

PCNA proliferating cell 1 nuclear antigen 


\begin{tabular}{|c|c|}
\hline PDGF & platelet-derived growth factor \\
\hline PDK-1 & phosphoinositide-dependent kinase 1 \\
\hline $\mathrm{PH}$ & Pleckstrin homology domain \\
\hline $\mathrm{PI}(3,4) \mathrm{P}_{2}$ & phosphatidylinositol-3, 4-bisphosphate \\
\hline PIP3 & phosphatidylinositol-3,4,5-trisphosphate \\
\hline PI3K & Phosphatidylinositide 3-kinase \\
\hline PI3KR2 & Phosphoinositide-3-kinase, regulatory subunit $2 \beta$ \\
\hline PKA & Protein kinase A \\
\hline PKB & Protein kinaes $\mathrm{B}$, or Akt \\
\hline $\mathrm{PKC}$ & Protein kinase $\mathrm{C}$ \\
\hline PKG & Protein kinase $\mathrm{G}$ \\
\hline PMSF & phenylmethylsulfonyl fluoride \\
\hline PP1, PP5 & Protein phosphatase 1,5 \\
\hline PRAS40 & Proline-rich Akt substrate of $40 \mathrm{kDa}$ \\
\hline PRX2 & Peroxiredoxin 2 \\
\hline PTEN & Phosphatase and tensin homologue \\
\hline PVDF & Polyvinylidene fluoride \\
\hline Raptor & regulatory associated protein of TOR \\
\hline $\mathrm{Rb}$ & Reinoblastoma protein \\
\hline $\mathrm{RC}$ & Reverse complementary \\
\hline ROS & Reactive oxygen species \\
\hline RT-PCR & Reverse transcriptase polymerase chain reaction \\
\hline S6K & 40S Ribosomal protein S6 kinase \\
\hline SDS & Sodium dodecyl sulfate \\
\hline
\end{tabular}




\begin{tabular}{|c|c|}
\hline SMAD & $\begin{array}{l}\text { homologs of both the Drosophila protein, mothers against } \\
\text { decapentaplegic (MAD) and the Caenorhabditis elegans protein SMA } \\
\text { (from gene sma for small body size). }\end{array}$ \\
\hline SOD & Superoxide dismutase \\
\hline TAE & Tris base, acetic acid and EDTA \\
\hline TBE & Tris base, boric acid and EDTA \\
\hline TBST & Tris-buffered saline with tween 20 \\
\hline TEMED & N,N,N',N'-Tetramethylethane-1,2-diamine \\
\hline TGF- $\beta$ & Transforming growth factor $\beta$ \\
\hline $\mathrm{TMB}$ & Tetramethylbenzidine \\
\hline TOR & Target of rapamycin \\
\hline TORC1, 2 & TOR complex 1,2 \\
\hline Tris & Tris (hydroxymethyl) aminomethane \\
\hline TRX1 & Thioredoxin 1 \\
\hline $\mathrm{TSC}$ & tuberous sclerosis \\
\hline rRNA & Ribosomal RNA \\
\hline UTR & Untranslated region \\
\hline UV & Ultraviolet \\
\hline VEGF & Vascular endothelial growth factor \\
\hline
\end{tabular}




\section{List of Figures}

Figure Freezing damages and protective effects of a cryoprotectant

$1.1 \quad$ (glucose)

Figure Glucose assay kit standard curve monitored at $500 \mathrm{~nm}$.

2.1

Figure Regulation of cell cycle progression

3.1

Figure Effects of $24 \mathrm{~h}$ freezing and $8 \mathrm{~h}$ thawing on Cdk protein levels as

3.2 well as total phosphorylation of Cdks on Thr14/Tyr15 (p-Cdk $\mathrm{T} 14 / \mathrm{Y} 15)$ in wood frog liver.

Figure Effects of $24 \mathrm{~h}$ anoxia exposure and $4 \mathrm{~h}$ of aerobic recovery on Cdk 66 3.3 protein levels as well as total phosphorylated Cdks Thr14/Tyr15 (pCdk T14/Y15) in wood frog liver.

Figure Effects of $40 \%$ dehydration and rehydration on protein levels of $\quad 67$

3.4 Cdks as well as total phosphorylated Cdks Thr14/Tyr15 (p-Cdk $\mathrm{T} 14 / \mathrm{Y} 15)$ in wood frog liver.

Figure Effects of $24 \mathrm{~h}$ freezing and $8 \mathrm{~h}$ thawing on protein levels of cyclins 68 3.5 in wood frog liver.

Figure Effects of $24 \mathrm{~h}$ anoxia exposure and $4 \mathrm{~h}$ aerobic recovery on protein 69 3.6 levels of cyclins in wood frog liver.

Figure Effects of $40 \%$ dehydration and rehydration on protein levels of $\quad 70$ 3.7 cyclins in wood frog liver.

Figure Effect of freezing, anoxia and dehydration stresses on the relative 71 3.8 phosphorylation state of Cdc25a phosphorylated on Ser76 (pCdc25a S76) and Cdc25c phosphorylated on Ser216 (p-Cdc25c S216) in liver of wood frogs. 
Figure Effect of freezing, anoxia and dehydration stresses on the relative

3.9 phosphorylation state of p21 phosphorylated on Thr145 (p-p21

T145) and p27 phosphorylated on Thr 187 (p-p27 T187) in wood

frog liver.

Figure $\quad$ Activation of Akt 96

4.1

Figure Effects of $24 \mathrm{~h}$ freezing and $8 \mathrm{~h}$ thawing on protein levels of Akt as $\quad 97$

4.2 well as relative phosphorylation of Akt at Ser-473 and Thr-308 in wood frog liver, skeletal muscle, kidney and heart.

Figure Effects of $24 \mathrm{~h}$ anoxia and $40 \%$ dehydration on protein levels of

4.3 Akt and relative contents of phospho-Akt Ser-473 and Thr-308 in wood frog liver.

Figure Effects of $24 \mathrm{~h}$ freezing and $8 \mathrm{~h}$ thawing on the nuclear content of

4.4 Akt protein and the relative levels of phospho-Akt Ser-473 and Thr308 in wood frog liver.

Figure Effects of $24 \mathrm{~h}$ freezing and $8 \mathrm{~h}$ thawing on protein levels of PDK-1 103

4.5 and relative phosphorylation of PDK-1 at Ser-241 in wood frog liver.

Figure $\quad$ Effects of $24 \mathrm{~h}$ freezing and $8 \mathrm{~h}$ thawing on PTEN in wood frog 104 4.6A liver.

Figure Homology tree for wood frog PTEN partial amino acid sequence 105 4.6B compared with multiple species.

Figure Partial amino acid sequence (141 residues) of wood frog PTEN

4.6C aligned with partial PTEN (or PTEN-like) sequences of multiple species.

Figure Effects of $24 \mathrm{~h}$ freezing and $8 \mathrm{~h}$ thawing on transcript levels of

4.7 miR-26a, miR-126 and miR-217 in wood frog liver.

Figure $\quad$ Effects of $24 \mathrm{~h}$ freezing and $8 \mathrm{~h}$ thawing on total FOXO1 protein 108 
4.8 and phospho-FOXO1 Ser-256 levels in wood frog liver.

Figure TORC1-dependent mRNA translation initiation. 130

5.1

Figure Effects of $24 \mathrm{~h}$ freezing and $8 \mathrm{~h}$ thawing on the total TOR protein 132

5.2 and phosphorylation state of TOR at Ser-2448 level in liver, muscle, heart and kidney of wood frogs.

Figure Effects of 24h freezing and 8h thawing on the total Raptor protein 134

5.3 levels and phosphorylation state (Ser-863) of Raptor in liver, muscle, heart and kidney of wood frogs.

Figure Effects of $24 \mathrm{~h}$ freezing and $8 \mathrm{~h}$ thawing on the protein levels of G $\beta \mathrm{L} \quad 136$

5.4 in liver, muscle, heart and kidney of wood frogs.

Figure Effects of 24h freezing and 8h thawing on total 4EBP protein 137

5.5 levels, and relative phosphorylation (Thr-45) of 4EBP in liver, muscle, heart and kidney of wood frogs.

Figure $\quad$ Effects of $24 \mathrm{~h}$ freezing and $8 \mathrm{~h}$ thawing on total S6K protein levels

5.6 and relative phosphorylation (Thr-389) of S6K in liver, muscle, heart and kidney of wood frogs.

Figure Effects of $24 \mathrm{~h}$ freezing and $8 \mathrm{~h}$ thawing on the relative

5.7 phosphorylation levels of TSC2 (Ser-939) and PRAS40 (Thr-246)

in liver, muscle, heart and kidney of wood frogs.

Figure Effects of $24 \mathrm{~h}$ freezing and $8 \mathrm{~h}$ thawing on P-TOR (Ser2448):

5.8 Raptor interactions liver, muscle, heart and kidney as assessed by co-immunoprecipitation (CO-IP).

Figure Effects of freeze-thaw and glucose injection on total protein levels

6.1 of catalase, PRX2, SOD1, SOD2 and TRX1 in wood frog liver.

Figure Effects of freeze-thaw and glucose injection on relative total protein 168

6.2 levels of catalase, PRX2, SOD1, SOD2 and TRX1 in wood frog skeletal muscle. 
Figure Effects of glucose injection on antioxidant capacity in liver and

6.3 muscle of wood frogs.

Figure Effects of freeze-thaw (A) and glucose injection (B) on relative

6.4 total protein levels of Nrf2 in wood frog liver and skeletal muscle.

Figure Effects of freeze-thaw and glucose injection on total protein levels

6.5 of GSK3 $\beta$ in wood frog liver and muscle.

Figure Effects of freeze-thaw and glucose injection on the relative amount 174

6.6 of phospho-GSK3 3 (Ser-9) detected in wood frog liver and muscle.

Figure EMSA and ELISA-type transcription factor-DNA binding assays. 176

6.7

Figure Effects of freeze-thaw and glucose injection on transcript level of 178 6.8 gstal in wood frog liver and muscle. 


\section{List of Tables}

Table 2.1 Antibody list $\quad 39$

Table 2.2 Primer list 40

Table 6.1A Glucose assay kit interference test 166

Table 6.1B Plasma glucose levels (mM) after saline or glucose injections. 166 
Chapter 1 General Introduction 


\section{Freezing stress and freeze tolerance}

Seasonal cold has imposed the challenge of freezing stress on many organisms. Those without proper freezing survival adaptations (i.e. freeze intolerant species) usually face multiple forms of lethal damage from freezing exposure. When experiencing freezing stress, several types of pressure and/or damage can occur: (a) intracellular ice formation can destroy subcellular compartmentation and structure; (b) formation of the extracellular ice can cause physical damage to organs and rupture blood vessels; (c) accumulation of ice crystals in the extracellular environment takes pure water out of cells, creating an osmotic stress; (d) solidification of the blood plasma halts blood circulation, triggering ischemia stress; (e) vital events involving muscle movement including heart beat and breathing as well as those related to nerve activity are halted (Storey, 1990; Rubinsky et al., 1987). Generally, damage cause by freezing can be categorized into two major groups: physical injuries to cell/tissues caused by ice formation, and interruption of normal cellular processes due to changes in energy metabolism caused by the consequences of freezing (e.g. anoxia, ischemia, cellular dehydration) (Fig. 1.1) (Storey and Storey. 2013).

In order to survive exposure to subzero temperatures over the winter months, organisms have developed a variety of adaptations over the course of evolution. Some can escape to thermally buffered environments that will not freeze by migration or hibernation under water or underground below the frost line (Storey, 2004a). Others have developed physiological and biochemical adaptations that provide cold hardiness so that they can endure subzero temperatures. Many organisms use freeze avoidance strategies that allow them to supercool to low subzero temperatures by using various antifreeze 
strategies to prevent their body fluids from freezing. Oppositely, a wide variety of invertebrates and vertebrates including many insect species and several amphibian and reptile species have developed freeze tolerance (Storey and Storey, 1988; Sinclair et al., 2003). Freeze tolerant organisms endure the freezing of body fluids in extracellular spaces which can cause a conversion of as much as $65-70 \%$ of total body water into ice (Costanzo et al., 1993; Layne, 1995a). While formation of ice is allowed in freeze tolerant organisms, strategies for limiting the size and location of the ice are necessary, for unrestricted ice growth will cause lethal damage to the system. Freeze tolerant organisms usually utilize extracellular ice nucleating agents (INA) to manage ice growth in the extracellular spaces (Storey and Storey, 2004a). These INAs trigger ice growth at high subzero temperatures allowing rates of ice growth to be minimized and leaving enough time to activate cell preservation adaptations; without this, organisms can face severe damage from instant ice accumulation if freezing begins from a deeply supercooled state (Claussen et al., 1990; Storey and Storey, 2004a). INAs can also direct ice formation in large extra-organ spaces such as bladder or the abdominal cavity as opposed to more restricted spaces such as within capillaries where damage due to ice expansion can be very serious (Storey and Storey, 2004a).

Freezing stress is typically accompanied by cellular dehydration and oxygen deprivation (Storey and Storey, 2004a). Ice formation in extracellular spaces creates high osmolality of extracellular fluids, which consequently places hyperosmotic stress on cells that draws water out, while also increasing in solute and ion concentrations within cells. While having positive effects such as reducing the probability of intracellular ice formation, dehydration leads to abnormal elevation of the ionic strength in body fluids, 
which may interrupt normal cellular and tissue functions. Freezing-induced dehydration also causes physical shrinkage of cell volume that may damage membrane bilayer structure. To help resist osmotic injury, freeze tolerant organisms use small molecules called cryoprotectants that are accumulated in high concentrations. Cryoprotectants have colligative effects that help to limit the reduction of cell volume due to the loss of water. Some cryoprotectants are small proteins with high solubility in concentrated solutions that can also move across the plasma membrane. Most cryoprotectants are small carbohydrates with high solubility. Glucose is the cryprotectant used by most freeze tolerant frogs including the wood frog (Rana sylvatica), the spring peeper (Pseudacris crucifer) and the chorus frog (Pseudacris triseriata) (Storey and Storey, 1984, 1986a). Glycerol is used as a cryoprotectant by gray tree frogs (Hyla versicolor, H. chrysoscelis) and the Siberian salamander (Salamandrella keyserlingii) (Berman et al, 1984; Storey and Storey, 1986a; Layne, 1999) as well as by huge numbers of freeze tolerant and freeze avoiding insects and other arthropods (Storey and Storey, 1988, 2012; Joanisse and Storey, 1994b,c).

Solidification of blood plasma triggers an ischemic state for cells where oxygen and fuel transportation are disrupted. As a result, cells are usually exposed to anoxia stress during whole body freezing. Therefore, without oxygen, ATP (Adenosine triphosphate) production must rely on anaerobic respiration pathways and must depend on the internal carbohydrate fuel reserves of cells. Both of these factors restrict the energy availability, and thus a new equilibrium needs to be established between energy production and consumption. In order to survive, energy expenditure needs to be reprioritized to support both basal metabolism as well as the necessary cell preservation 
pathways (Storey and Storey, 2004a; Zhang et al., 2011). As a result, this energy rearrangement and long term viability in the frozen state ultimately requires a net suppression of metabolic rate and entry into a hypometabolic state. Furthermore, a freezethaw cycle essentially creates an ischemia-reperfusion situation, which has been recognized as a major source of oxidative stress in mammalian systems (Campos et al., 1993; Downey, 1990; Fuller et al., 1988; Ruuge et al., 1991; Traystman et al., 1991). Therefore, freeze tolerant organisms usually experience rapid reactive oxygen species (ROS) production upon freeze-thaw conditions. Thus, antioxidant strategies must be included in their stress adaptations.

\section{General stress responsive cascades}

The main focus of adaptation for freeze tolerant animals is to slow or shut down various 'high energy cost' biological events during the energy-restricted frozen state while still enhancing those that are required for freezing survival. Biological adjustments that happen as freeze tolerant organisms transition into the frozen state include metabolic rate depression (MRD), cryoprotectant synthesis, freeze-responsive signal transduction and gene expression, and differential regulation of many functions including growth and proliferation (cell quiescence, senescence, cell cycle arrest, etc.), and cell survival (apoptosis and autophagy) (Storey, 1987a; Cowan and Storey, 2003; Storey 2004b). Under the control of signal transduction pathways, cells respond to environmental change to adjust themselves to new circumstances. Stress signals can trigger changes to the existing protein/enzyme machinery in cells (e.g. via posttranslational modifications such as phosphorylation), as well as changes in gene expression to alter the type and/or 
amount of cellular proteins/enzymes. Thus, when a stress occurs, it is converted into various biological signals, following cell signaling pathways and eventually reaches the transcriptional and translational levels to trigger regulatory effects on gene and protein expression, which subsequently alter cell proliferation, growth, survival and metabolism, etc. That means the key events lie at two distinct but related levels of cellular organization - cell signaling pathways versus transcriptional and/or translational regulation.

\section{The freeze-tolerant wood frog}

The wood frog (R. sylvatica) exhibits well-developed natural freeze tolerance that includes both physiological and biochemical adaptations that enable survival of seasonal subzero temperatures. Early studies showed that wood frogs are able to survive several weeks of freezing exposure (studies explored under various parameters such as duration of freezing exposure, temperature and frog developmental stages) (Storey and Storey, 1984, 1986a, 2004a). Due to its good freeze tolerance capability, the wood frog has been used as the primary animal model for studies of vertebrate freeze tolerance. The complexity of freezing stress (with its multiple accompanying stresses such as dehydration and anoxia) determines that freeze tolerance by wood frogs must include adaptations for surviving oxygen depletion, cellular water loss as well as oxidative stress, among others. Freeze tolerance adaptations by wood frogs are usually achieved by a set of well-tuned regulatory controls at the molecular level, starting from cell signal transduction to gene expression, which are ultimately reflected by responses of cellular events under stress conditions. Therefore, choosing wood frogs as the model of my thesis 
enabled the possibility of depicting a comprehensive view of an environmental stress responsive network. Also, in wood frogs, whole body freezing progresses at a relatively slow rate as compared with small freeze tolerant species such as the insects. This provides a good opportunity for monitoring the regulation of gene expression and other adaptive changes based on a detailed time course of freeze-thaw exposure.

\section{Overview of freeze tolerance by wood frogs}

Wood frogs have perfected a set of physiological adaptations dealing with freezing stress. As a freeze tolerant organism, formation of ice crystals on an acceptable level is allowed in wood frogs when experiencing freezing exposure. It has been reported that they can still survive when $\sim 65-70 \%$ of total body water is converted into extracellular ice (Costanzo et al, 1993). Initiation of extracellular ice formation is the first important step in freeze tolerance. The goal of this event is to commence ice nucleation, regulate the sites of ice formation, and minimize the speed of ice accumulation while maximizing the time to proliferate various adaptation steps. Wood frogs initiate ice nucleation just below the freezing point $\left(\mathrm{FP}\right.$; about $\left.-0.5^{\circ} \mathrm{C}\right)$ of their body fluids using (a) environmental ice to seed nucleation across the skin, and/or (b) ice nucleating bacteria on the skin or in the gut, and/or (c) INAs in plasma (Layne et al, 1990; Lee et al, 1995; Storey, 1990).

Wood frogs are capable of enduring ice formation in extracellular and extra-organ fluid. This leads to partial dehydration of cells and organs, but reduces the potential for damage to cells by preventing the formation of intracellular ice. In order to limit the amount of cellular dehydration that occurs and the amount of ice that forms, wood frogs 
synthesize, accumulate and distribute low molecular weight cryoprotectants (mainly glucose) to increase the osmolality of body fluids (Storey and Storey, 1984). This occurs rapidly during the first hours of freezing so that cryoprotectant can be distributed before freezing halts blood circulation.

Ischemia stress induced by the solidification of blood plasma during freezing cuts off the interorgan transport of oxygen, fuels, metabolic wastes, etc. However, wood frogs can endure such anoxic conditions (Holden and Storey, 1997). One aspect of survival is metabolic rate depression that reduces cellular energy demands to a level that can be fueled by the ATP generated from glycolysis alone (Cowan and Storey, 2001). When frogs thaw, however, they are exposed to oxidative stress as well, which is caused by a rapid rise in ROS when oxygen supply to tissues rises rapidly when breathing and blood flow are reinstated. To deal with this, wood frogs keep high activities of antioxidant enzymes in their tissues and/or change these activities in response to freeze/thaw (discussed in detail later) (Joanisse and Storey, 1996).

\section{Cryoprotection in wood frogs}

Wood frogs accumulate glucose as their cryoprotectant. As mentioned above, a main function of cryprotectant is to lower the difference of solute concentrations between intra- and extracellular environments during freezing exposure, thereby preventing cells from being overly dehydrated. Typical characteristics of cryoprotectants include (a) a low molecular weight, (b) high solubility at high concentrations, and (c) being easy to synthesize. In addition, they also (d) don't interfere with protein/enzyme functions (ie. are compatible solutes), and (e) can be readily transported across cell membranes (Storey 
and Storey, 2004a). During freezing exposure, wood frogs accumulate up to $300 \mathrm{mM}$ glucose cryoprotectant in plasma and tissues (compared with $\sim 5 \mathrm{mM}$ in control frogs) (Storey and Storey, 2004a). Glucose production is stimulated within 2-5 min when frogs start to freeze, rises rapidly over the initial hours, stays elevated during freezing and then is clearly slowly over several days after thawing (Storey and Storey, 1985, 1986a, b; Storey, 1987a). Glucose is produced from large reserves of glycogen in liver and is reconverted to glycogen again after thawing (Storey, 1987a). Notably, frogs endure hyperglycermia that is many-fold higher than can be survived by human diabetics.

Wood frogs build up high levels glycogen in liver during late summer and early fall feeding (Storey and Storey, 1984). This glycogen is the primary (perhaps sole) source for cryoprotectant synthesis as there is little indication of glycogen depletion in other organs in response to freezing. Glucose synthesis in wood frogs is triggered by extracellular ß-adrenergic signals that activate intracellular protein kinase A (PKA)dependent signal transduction to stimulate glycogenolysis and the short biosynthesis pathway (glucose-1-phosphate to glucose-6-phosphate (G6P) to glucose) (Storey, 1987a; Holden and Storey, 1996). The pathway is subject to regulation from multiple enzymes and other pathways. For example, since the last intermediate G6P can also serve as a substrate for other pathways, it is under tight control in wood frog liver to ensure high glucose production. One example is that the activity of G6PDH (glucose-6-phosphate dehydrogenase) that directs G6P into the pentose phosphate pathway is inhibited in the liver of frozen wood frogs (Cowan and Storey, 2001). The key enzymes involved in glycogen breakdown are glycogen phosphorylase (GP), phosphoglucomutase and glucose-6-phosphatase. GP cleaves glucose-1-phosphate units off the glycogen polymer 
and it highly activated within minutes when freezing begins, leading to a rapid elevation of liver and plasma glucose level (Storey and Storey, 1985). GP is activated by protein phosphorylation (PKA phosphorylates phosphorylase kinase which in turn phosphorylates GP) to convert GP from its inactive " $b$ " form to the active "a" form. GPa levels rise quickly as freezing exposure extends (Storey, 1987b; Storey and Storey, 1989).

After production in liver, glucose distribution is the next critical step for cryoprotectant accumulation in wood frog. Glucose transport in vertebrates is carriermediated by a family of transmembrane glucose transporters (GLUTs). In most frog tissues, glucose transport is unidirectional from plasma into cells and is conducted by GLUT1, GLUT3 or the insulin-sensitive GLUT4. However, liver has the bidirectional transporter GLUT2 that allows glucose to be imported or exported as needed (Pessin and Bell, 1992) and during freezing mediates the rapid export of glucose to other tissues. Studies showed that wood frog liver possesses higher amount of all GLUTs (especially GLUT2) than freeze intolerant leopard frogs (Rana pipiens) (King et al., 1993). It also appears that the glucose transportation capacity as well as GLUT2 protein levels in wood frog liver varies according to season with both increased significantly in autumn frogs as compared with spring animals (King et al., 1995).

Freezing stress responsive signal transduction in wood frog

Various signal transduction pathways are involved in achieving the freezing responsive adaptations in wood frog. With the help of contemporary genomic and proteomic technologies, studies of the molecular mechanisms underlying freezing 
survival can be conducted at a high throughput level. Previous studies of gene expression patterns in wood frogs during freezing using cDNA library or DNA microarray screening revealed up-regulated expression levels of several genes involved in cell signal transduction. Examples included PI3K (phosphoinositide 3-kinase), CK2 (casein kinase 2), PP5 (protein phosphatase 5) (Storey, 2004b). In addition, a recent proteomic study using LC-MS (liquid chromatography - mass spectrometry) discovered elevated expression levels of 14 proteins in winter frogs. Not surprisingly, those also included cell signaling proteins such as PKC (protein kinase C) and PP1 (protein phosphatase 1) (Kiss et al., 2011). These studies suggest that stress responsive signal transduction is a crucial part of freezing survival in wood frogs.

As briefly mentioned above, glucose production by wood frog liver is mediated by PKA signaling. Previous studies reported that $\beta$-adrenergic receptors were activated in frozen frogs, which subsequently triggered cyclic AMP (cAMP) production, leading to PKA activation. PKA then activates phosphorylase kinase that in turn activates GP (converting it to active GPa) to trigger the breakdown of glycogen in liver (Holden and Storey, 1996; 2000) and increased glucose production within minutes (Storey and Storey, 1986b). At the same time, catabolic pathways such as glycolysis are slowed down or shut off (Cowan and Storey, 2001; Storey, 1987a). In addition, to maintain high levels of glucose for cryoprotection, another crucial need is to override the normal homeostatic controls on glucose levels that in control frogs keep plasma glucose levels at $\sim 5 \mathrm{mM}$. Although the molecular mechanism behind this has yet to be fully elucidated, regulation of PP1 appears to be involved. The phosphatase removes phosphate groups from GP to reconvert it to $\mathrm{GPb}$, the inactive form. Previous studies proposed that during 
freezing exposure PP1 was localized away from glycogen particles, leading to a reduced ability to dephosphorylate GP (MacDonald and Storey, 1999).

Other signaling pathways are also involved in wood frog freezing survival, including MAPKs (mitogen-activated protein kinases), cGMP-dependent PKG (protein kinase $\mathrm{G}$ ), as well as $\mathrm{Ca}^{2+}$ and inositol trisphosphate dependent PKC (protein kinase C) (Cowan and Storey, 2003; Holden and Storey, 1996). The involvement of stress responsive MAPK signaling cascades in freeze tolerance suggests that cellular processes that are common targets of MAPK action, such as the cycle cell and apoptosis, could also be subject to freeze responsive regulation. In addition, up-regulation of PI3K (Phosphatidylinositide 3-kinase) expression levels in liver of frozen frogs, as determined by DNA array screening, suggested a potential connection with Akt (or PKB, Protein kinase B) signaling (Storey, 2004b). This suggests that freeze responsive signal transduction may also serve as a switch for regulating various adaptations of cellular events, such as energy metabolism and antioxidant defense.

Other established freeze-thaw responsive molecular adaptations in wood frogs

Years of research on freeze tolerance in wood frogs have identified a wide range of genes that are subject to freeze-thaw regulation. Protein chaperones are one example. They are a set of proteins that interact with the target proteins to protect them against aggregation caused by unfolding, and to correct protein misfolding (Gething and Sambrrok, 1992; Feder and Hofmann, 1999). HSPs (heat shock proteins) and GRPs (glucose-regulated proteins) are two major families of protein chaperones. The former are well known for their stress responsive nature. A variety of stress conditions are able to 
induce chaperones, such as heat, oxygen deprivation, UV radiation, etc. It has also been proposed in freeze intolerant animal models that HSPs are responsive to cold stress (Yiangou et al., 1997; Kimura et al., 1998; Nielsen et al., 2005; Qin et al., 2005). Furthermore, a proteomics study has shown that the HSP70 level was higher in the winter wood frogs than summer animals (Kiss et al., 2011). Emerging evidence has been reveal that HSPs proteins are deeply involved in freezing survival by a range of cold tolerant species (Storey and Storey, 2011, 2012).

As mentioned above, oxidative stress is one major pressure that freeze tolerant organisms need to deal with, due to the nature of freeze-thaw induced ischemiareperfusion, as well as high glucose contents. Previous studies revealed that freeze-thaw exposure induced increases in the activities of several antioxidant enzymes in wood frog tissues (Joanisse and Storey, 1996a). Also, wood frog liver showed a considerably higher antioxidant defense capacity than that of freeze intolerant leopard frogs $(R$. pipiens), suggesting that antioxidant defense is an integral part of the freezing survival strategy (Joanisse and Storey, 1996a).

Reversible protein phosphorylation and microRNA (miRNA) dependent posttranscriptional regulation

The main theme of stress survival during hypometabolism is energy preservation, which includes reprioritizing energy expenditure (overall suppression of energy consuming cellular processes but selective activation of stress survival adaptations). Utilizing strategies to enhance energy efficiency in regulating protein or enzyme functions is a crucial step in achieving this. With limited energy production possible in 
the frozen state, major changes in transcription and translation cannot be accomplished. Therefore, alternatives that are both energy efficient, functionally effective, and readily reversible are put to use. Posttranslational control of protein/enzyme function via reversible protein phosphorylation is one major strategy that can be used. Posttranscriptional regulation of mRNA transcripts via the actions of microRNA is another. These can be used by freeze tolerant organisms to suppress energy expensive processes such as cell proliferation (Biggar et al., 2009; Zhang $\boldsymbol{e t}$ al, 2013) and to activate cell preservation pathways (Storey and Storey, 2004a). Both of these mechanisms are able to regulate protein function without the need for extensive protein synthesis and/or degradation.

Reversible protein phosphorylation is one of the most commonly seen protein posttranslational modifications. It regulates the function of proteins by covalently adding phosphate onto serine, threonine or tyrosine residues. Phosphorylation events are catalyzed by protein kinases and reversed by protein phosphatases. Protein phosphorylation and dephosphorylation control the function of proteins (e.g. enzyme activity) largely by introducing conformational changes to them. The role of protein phosphorylation in cold hardiness is well documented in regulating kinase-based cell signaling pathways (e.g. MAPK cascades, Akt pathway, AMPK or AMP-activated protein kinase pathway, etc.) and activities of enzymes involved in energy metabolism (e.g. G6PDH) (Joanisse and Storey, 1994b, 1994c; Russell and Storey, 1995; Muise and Storey, 1997; Storey and Storey, 2004b; Pfister and Storey, 2006; Rider et al., 2011; Holden and Storey, 2011; Dieni et al., 2012). As mentioned previously, phosphorylation control of enzymes is particularly important to the production of glucose 
as the cryoprotectant wood frogs. Not only GP and G6PDH (mentioned above) but other glucose-related enzymes are subject to reversible protein phosphorylation control during freezing including GSK3 (glycogen synthase 3). This kinase, although first identified in association with glycogen synthase, is now known to have wide ranging control over many cell functions including antioxidant defense. Freeze responsive regulation of protein/enzyme function by reversible phosphorylation status is one of the core topics in the various studies presented in this thesis.

In recent years, identification and exploration of the regulatory roles of noncoding microRNAs in the post-transcriptional (or pre-translational) control of gene expression has grown explosively. In fact, recent studies proposed that miRNA is predicted to be involved in regulating over $60 \%$ of protein-coding genes (Ebert and Sharp, 2012). In animals, mature miRNAs mainly target the seed region of the 3-UTR (untranslated region) of mRNA transcripts and form a silencing complex (miRNAinduced silencing complex or miRISC), which ultimately guides mRNA to either argonaute endonuclease-dependent RNA degradation (if there is perfect binding between miRNA and mRNA) or reversible RNA granule sequestration (imperfect binding) (Bartel, 2009). Differential expression of miRNAs was recently identified as a response to anoxia stress in turtles (Zhang et al., 2013). Furthermore, a pilot study showed changes in the expression levels of two miRNAs that are involved in regulating p53 and Akt pathways in response to freezing stress in wood frogs (Biggar et al., 2009) and changes in miRNA expression also occur in freeze tolerant insects (Courteau et al., 2012; Lyons et al., 2013), strongly indicating that posttranscriptional regulation of 
mRNA via miRNA action will have a significant role to play in freezing survival by wood frogs.

\section{Objectives and hypotheses}

Understanding the molecular mechanisms is the key to fully deciphering the mystery of natural freeze tolerance and applying this knowledge to various medical areas such as improving or developing cell, tissue and organ freezing preservation mechanisms to benefit goals such as transplantation. Given that mammalian species is incapable of surviving prolonged freezing exposure, the present thesis uses the freeze tolerant wood frog as the animal model of interest. Furthermore, the accumulation of glucose as the cryoprotectant in wood frogs makes this animal very interesting as a model for diabetes research since frogs endure and regulate extremely high glucose levels without showing the damaging effects of high glucose that are common in diabetic patients.

In order to gain a comprehensive view of wood frog freeze tolerance and the complex regulatory and signalling networks that support freezing survival, the present thesis has been divided into three major areas, all of which are related closely to the consequences of freeze-thaw exposure in wood frogs:

1. Regulation of major cellular events (cell cycle and protein synthesis)

2. Roles of the related signal transduction pathway (Akt pathway)

3. Effect of glucose on gene expression and antioxidant defense 
Objective 1 - Determine how the cell cycle responds to freeze-thaw in liver, identify the intracellular regulatory mechanisms involved, and the stress stimuli that act as triggers (e.g. anoxia or dehydration). (Chapter 3)

\section{Hypothesis:}

Cell cycle progression is inhibited in liver of wood frogs during freezing and in response to one or both component stresses of freezing (anoxia, dehydration).

As described above, cell proliferation is a highly energy expensive process. I predicted that the cell cycle would be suppressed during freezing due to limited energy availability in the frozen hypometabolic state. Chapter 3 tests the hypothesis by analyzing changes in protein expression levels of key cell cycle components in response to freezing, anoxia or dehydration stresses in wood frog liver. Immunoblotting was used to investigate protein expression of Cdc 2 (cell division control protein 2 homolog), Cdks (Cyclin-dependent kinase) (2, 4 and 6) and cyclins (A, B1, D1 and E) as well as the phosphorylation states of Cdks (Thr14/Tyr15), the phosphatases Cdc25a (Ser76) and Cdc25c (Ser216), and the Cip/Kip (CDK interacting protein/Kinase inhibitory protein) Cdk inhibitors p21 (Thr145) and p27 (Thr187).

Objective 2 - Analyze the regulation and response of the Akt signaling pathway in multiple tissues (liver as a focus) in response to freeze-thaw (Chapter 4)

Hypothesis: 
Differential regulation of the Akt signalling pathway mediates freeze specific responses in wood frog liver including cryoprotectant synthesis, protein translation, and anti-apoptosis actions.

The insulin responsive Akt pathway is involved in controlling a wide variety of cellular processes including the cell cycle, cell preservation, protein synthesis and glucose metabolism. Due to the importance of glucose accumulation as a cryoprotectant in the wood frog during freezing and the normal role of Akt in mediating glucose use and glucose homeostasis in cells, I predicted that this central signalling pathway plays a critical role in wood frog freezing survival. Chapter 4 addresses the hypothesis by investigating gene and protein expression patterns and phosphorylation states of core protein components of Akt signalling in wood frog liver (with selected data on other tissues) in response to whole body freezing and thawing. The involvement of miRNA action in regulating gene expression patterns is also examined along with an analysis of FOXO1, a downstream target of Akt signaling that is important in the anti-apoptosis action of Akt. Furthermore, to examine the stress specific nature of Akt signaling, comparable responses by Akt signaling to two components of freezing (anoxia and dehydration) are evaluated.

Objective 3 - Determine the regulation and response of TORC1, a key factor in protein synthesis control, in multiple tissues in response to freeze-thaw (Chapter 5)

Hypothesis: 
TORC1 assembly and activity is strongly suppressed to minimize global protein synthesis in the frozen state.

Protein synthesis is a major ATP-consuming cell process that needs to be strongly suppressed when cellular energy is limiting and/or during excursions into hypometabolism. TORC1 (TOR complex1, or target of rapamycin complex 1)-dependent mRNA translation initiation plays a pivotal role in the regulation of protein synthesis and, by extension, in cell growth and proliferation. The TORC1 pathway is a major downstream target of Akt signalling and therefore, predictably, TORC1 responses should be integrated with those of Akt. Chapter 5 tests the hypothesis by investigating the expression of total TOR protein and its phosphorylation status as well as targets of TOR action including Ser-863 phosphorylated Raptor, Ser-939 phosphorylated TSC2 (tuberous sclerosis) and Thr-246 phosphorylated PRAS40 (proline-rich Akt substrate of $40 \mathrm{kDa}$ ) in response to whole body freezing of wood frog. In addition, cellular levels of phospho4EBP (eIF4E binding protein, or eukaryotic initiation factor 4E binding protein) and phospho-S6K (Ribosomal protein S6 kinase), proteins involved in regulating translation initiation complex assembly, were examined as downstream outcomes to TORC1dependent translational regulation. Furthermore, immunoprecipitation was used to monitor TORC1 assembly to explore the mechanism of TORC1-dependent translational control in under freezing stress.

Objective 4-Evaluate the regulatory effects of glucose on Nrf2-dependent antioxidant defense during wood frog freeze-thaw (Chapter 6) 
Hypothesis:

Antioxidant defenses, mediated by the Nrf2 transcription factor, are activated in wood frog liver and muscle in response to freeze-thaw; the response potentiated by high glucose effects on Nrf2 action.

As described above, wood frogs accumulate high levels of glucose as a cryoprotectant during freezing and glucose levels remain high for several days post-thaw. The consequences of high glucose for the resumption of normal metabolic functions and intracellular signaling have never been investigated. Of particular interest is the combination of ischemia-reperfusion (caused by freeze-thaw) and high glucose levels which can both lead to high oxidative stress in other systems. A strong response by antioxidant defense mechanisms is therefore predicted. The transcription factor Nrf2 (Nuclear factor (erythroid-derived 2)-like 2) binds to the ARE (antioxidant responsive element) in the promoter region of numerous antioxidant genes and regulates their expression. Therefore, the behaviour of Nrf2 over freeze-thaw and of antioxidant defense genes/proteins under its control was examined along with an analysis of glucose effects on the pathway. Starting from a study of the protein expression levels of key antioxidant enzymes using a multiplex assay, Chapter 6 focuses on the effects of glucose on the antioxidant response in muscle and liver of wood frogs over freeze-thaw. In addition to direct measurement of changes in relative antioxidant capacity in response to intraperitoneal injection of high glucose, the effect of glucose on antioxidant adaptations was also assessed in multiple ways, including its effect on the protein expression levels of 
antioxidant enzymes and the Nrf2 transcription factor, as well as glucose effects on the DNA binding activity of Nrf2 in vitro. 
Fig. 1.1

\section{Normal cell}
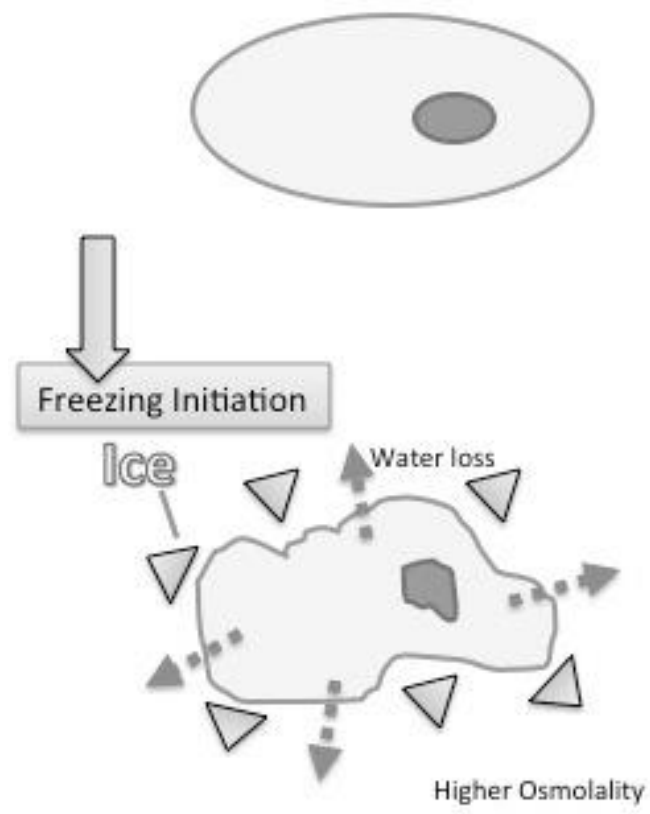

\section{No protection}
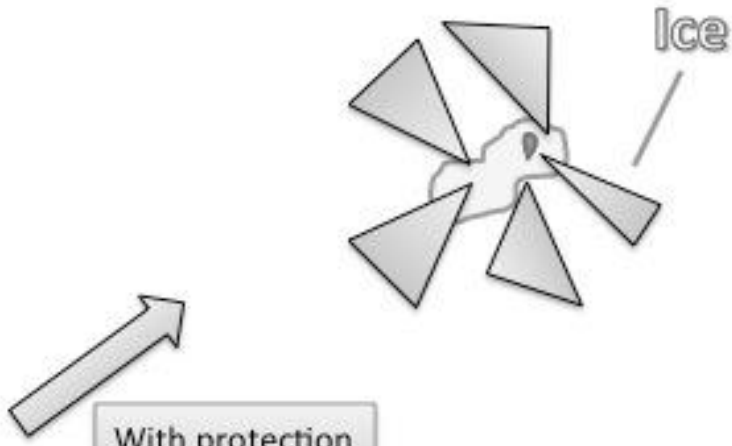

With protection

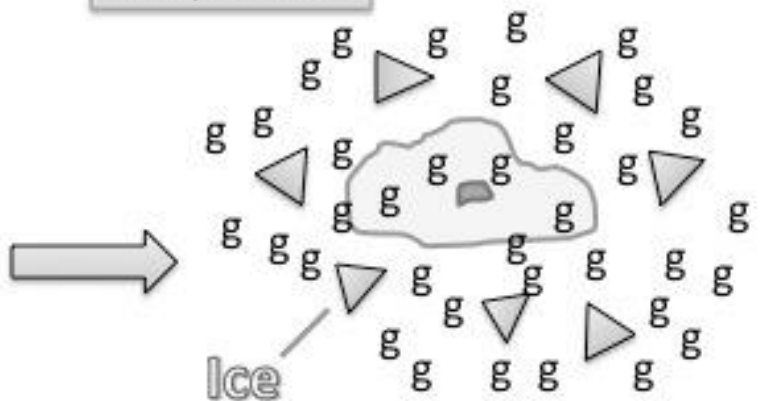

g: glucose (a colligative cryoprotectant)

Fig. 1.1 Freezing damages and protective effects of a cryoprotectant (glucose) 


\section{Chapter 2 Material and methods}


Animals (freezing-all chapters, dehydration and anoxia-Chapter $3 \& 4$, saline and glucose injection-Chapter 6)

Male wood frogs were collected from breeding ponds in the Ottawa area early in the spring. All frogs were washed in a tetracycline bath and were then placed in plastic boxes containing damp sphagnum moss; animals were held at $5^{\circ} \mathrm{C}$ for $1-2$ weeks before proceeding to experiments. Some frogs were sampled at this stage as the control animals.

For freezing exposure, frogs were placed in plastic containers with a layer of damp paper towels on the bottom and then placed at $-4.0^{\circ} \mathrm{C}$ in an incubator; a $45 \mathrm{~min}$ cooling period was allowed for frog body temperatures to chill to subzero values and nucleation to occur. Subsequently, temperature was raised to $-2.5^{\circ} \mathrm{C}$ and timing of a $24 \mathrm{~h}$ freezing exposure began. Half of the frogs were sampled after this freezing exposure and the others were returned to $5^{\circ} \mathrm{C}$ for $8 \mathrm{~h}$ thawing before sampling.

For anoxia experiments, plastic jars were prepared with a layer of damp paper towel on the bottom, which was wetted with distilled water that had previously been bubbled with $100 \%$ nitrogen gas. The lid of each jar had 2 ports; one connected to a gas line allowed flushing with nitrogen gas for $20 \mathrm{~min}$ and the second port vented the gas. Jars were held chilled on crushed ice throughout. Frogs were quickly placed in each jar, the lid was tightened, and gassing with $\mathrm{N} 2$ was continued for 30 min followed by closing the ports and sealing the jar with parafilm. Jars were placed in a $5^{\circ} \mathrm{C}$ incubator for $24 \mathrm{~h}$. After anoxia exposure, some jars were replaced on ice, the nitrogen gas line was reconnected, and then frogs were quickly sampled. Frogs in other jars were transferred to new jars with normal air and given a $4 \mathrm{~h}$ aerobic recovery period at $5^{\circ} \mathrm{C}$ before sampling. 
For dehydration experiments, frogs were weighed and transferred into large desiccator jars with a layer of silica gel desiccant on the bottom (a layer of dry sponge separated frogs from the desiccant). Jars were held at $5^{\circ} \mathrm{C}$ throughout. The weight of each frog was measured at intervals to determine the amount of body water lost. All frogs were dehydrated until $40 \%$ of total body water was lost and then half of the frogs were sampled. The others were transferred to containers with $0.5 \mathrm{~cm}$ distilled water on the bottom and sampled after full rehydration at $5^{\circ} \mathrm{C}$. The percentage of total body water loss was calculated using the following equation: (Mi-Md) / $\left(\mathrm{Mi} \times \% \mathrm{H}_{2} \mathrm{O}\right)$, where $\mathrm{Mi}$ is the initial mass of the animal, $\mathrm{Md}$ is the mass at each weighing and $\% \mathrm{H}_{2} \mathrm{O}$ is the percentage of total body mass that is water (for control wood frogs, $\% \mathrm{H}_{2} \mathrm{O}$ is $80.8 \pm 1.2 \%$ ).

A total of 37 frogs (mean body mass $=5.8 \pm 0.1 \mathrm{~g}$ ) were given intraperitoneal glucose injection to artificially elevate their cryoprotectant levels. Frogs were randomly separated into two groups for saline versus glucose injection. Frogs of the saline group were intraperitoneally injected with a volume of saline $(0.9 \% \mathrm{NaCl})$ equivalent to $8 \%$ of body mass. Frogs in the glucose group were injected with an equivalent volume ( $=8 \%$ of body mass) of $2 \mathrm{M}$ glucose (dissolved in saline). All frogs were held in a $5^{\circ} \mathrm{C}$ incubator for $2 \mathrm{~h}$ before being sampled. Besides muscle and liver, blood samples were also collected from the frogs of both groups using a disposable micropipette to remove blood from the severed aorta. Blood samples were mixed with EDTA (10 mM, dissolved in saline) immediately in order to prevent blood clotting. Blood samples were then centrifuged in an Eppendorf centrifuge at maximum speed for 5 min at $4^{\circ} \mathrm{C}$. Supernatants were collected as blood plasma. 
All tissue and plasma samples were stored at $-80^{\circ} \mathrm{C}$ for future use. All protocols regarding animal care, experimentation and euthanasia were conducted in accordance with the guidelines of the Canadian Council on Animal Care and had the prior approval of the Carleton University Animal Care Committee.

\section{Glucose assay (Chapter 6)}

A glucose assay kit (Cat\#: 1009582, Cayman Chemical, Michigan, USA) was used for measuring plasma glucose level. A standard curve was constructed using the standard glucose solutions provided with the kit (Fig. 2.1). The concentration of glucose in blood plasma samples wells ( $\mathrm{n}=6$ independent trials/experimental condition) was determined by comparison to the standard curve containing known concentrations of glucose. The assay was performed following the manufacturer's instructions. Briefly, for each reaction, $15 \mu \mathrm{l}$ of plasma sample (or standard solution) was mixed with $85 \mu 1$ of assay buffer and $100 \mu 1$ of enzyme mixture in a well of a 96-well microplate. Reactions were allowed to incubate for $10 \mathrm{~min}$ at $37^{\circ} \mathrm{C}$ and then absorbance was read on a microplate reader at $500 \mathrm{~nm}$.

Moreover, wells containing a known concentration of glucose $(0.69 \mathrm{mM})$ in addition to blood plasma samples (samples selected from the $6 \mathrm{~h}$ experimental condition) were combined into a single well and the glucose concentrations were measured. This was done in order to test for assay interference which may occur as a result of the in vivo environment of the wood frog. 


\section{Total protein isolation (all chapters)}

Samples of frozen tissues were homogenized 1:2 w:v in using a pre-chilled homogenization buffer [20 mM HEPES or 4-(2-hydroxyethyl)-1piperazineethanesulfonic acid, $\mathrm{pH} 7.4,200 \mathrm{mM} \mathrm{NaCl}, 0.1 \mathrm{mM}$ EDTA or Ethylenediaminetetraacetic acid, $10 \mathrm{mM} \mathrm{NaF}, 1 \mathrm{mM} \mathrm{Na}_{3} \mathrm{VO}_{4}, 10 \mathrm{mM} \beta-$ glycerophosphate] with a few crystals of PMSF (phenylmethylsulfonyl fluoride) and $1 \mu 1$ protease inhibitor (BioShop Canada Inc. Burlington, ON) added immediately before use. Homogenates were then centrifuged at $12,000 \mathrm{xg}$ at $4^{\circ} \mathrm{C}$ for $15 \mathrm{~min}$. Supernatants were collected as total soluble protein samples. Protein concentrations were determined in triplicate using the Bradford assay and standardized to $10 \mu \mathrm{g} / \mu \mathrm{l}$ by addition of small aliquots of homogenization buffer.

\section{Immunoblotting (all chapters)}

Aliquots of the $10 \mu \mathrm{g} / \mu \mathrm{l}$ total soluble protein samples from each experimental group were mixed with an equal volume of 2X SDS (Sodium dodecyl sulfate) buffer [100 $\mathrm{mM}$ Tris Base or tris (hydroxymethyl) aminomethane, 4\% w/v SDS, 20\% v/v glycerol, $0.2 \% \mathrm{w} / \mathrm{v}$ bromophenol blue, $10 \% \mathrm{v} / \mathrm{v} 2$-mercaptoethanol] to give a final protein concentration of $5 \mu \mathrm{g} / \mu \mathrm{l}$. Samples were then boiled for $5 \mathrm{~min}$ and stored at $-40^{\circ} \mathrm{C}$ for future use. SDS polyacrylamide gels were prepared; stacking gel composition was 5\% acrylamide $\mathrm{v} / \mathrm{v}$ in $\mathrm{pH} 6.8$ Tris buffer (1M Tris) with $0.1 \%$ SDS, $0.1 \%$ APS or ammonium persulphate, $0.1 \%$ TEMED whereas resolving gels were $10 \%$ acrylamide $\mathrm{v} / \mathrm{v}$ in $\mathrm{pH} 8.8$ Tris buffer (1.5M Tris), with $0.1 \%$ SDS, $0.1 \%$ APS, $0.1 \%$ TEMED (N,N,N',N'Tetramethylethane-1,2-diamine). Aliquots of tissue samples containing $20 \mu \mathrm{g}$ protein 
were loaded into wells and separated at $180 \mathrm{~V}$ for $45 \mathrm{~min}$ in a running buffer $(25 \mathrm{mM}$ Tris-base, $190 \mathrm{mM}$ glycine, $0.1 \% \mathrm{w} / \mathrm{v}$ SDS, $\mathrm{pH}$ 7.6). Proteins were then electroblotted onto PVDF (polyvinylidene difluoride) membrane by wet transfer in a transfer buffer ( 25 $\mathrm{mM}$ Tris $\mathrm{pH} 8.5,192 \mathrm{mM}$ glycine, $20 \% \mathrm{v} / \mathrm{v}$ methanol). Electroblotting was performed at a constant $0.16 \mathrm{~A}$ at $4{ }^{\circ} \mathrm{C}$ for $90 \mathrm{~min}$. After 20 min blocking with $5 \% \mathrm{w} / \mathrm{v}$ skim milk in TBST (Tris-buffered saline with Tween 20) (20mM Tris base, $150 \mathrm{mM} \mathrm{NaCl}, 0.05 \%$ Tween 20), membranes were washed with TBST and incubated with a primary antibody specific to the protein of interest $(1: 1000 \mathrm{v} / \mathrm{v}$ dilution, in TBST $)$ at $4^{\circ} \mathrm{C}$ overnight. Membranes were then washed with TBST and incubated with an HRP (horseradish peroxidase) -linked secondary antibody corresponding to the primary antibodies (antirabbit IgG or immunoglobulin G, Cat\#: APA002P, anti-goat IgG, Cat\#APA004P, BioShop Canada Inc., Burlington, ON) (1:8000 v/v dilution in TBST) at room temperature for $40 \mathrm{~min}$. After incubation with secondary antibody, membranes were washed again with TBST and visualized with enhanced chemiluminescence $\left(\mathrm{H}_{2} \mathrm{O}_{2}\right.$ and Luminol). All the antibodies used in the thesis are listed in Table 2.1.

Immunoblotting band pictues for each data chapter were presented as truncated images of the whole blot image. Refer to Fig. A1 of Appendix A for examples of complete blots.

\section{Co-Immunoprecipitation (Chapter 5)}

Samples of wood frog liver, muscle, heart and kidney from each experimental condition were homogenized using a medium salt lysis buffer $[10 \mathrm{mM}$ Trs $\mathrm{HCl}, 10 \mathrm{mM}$ $\mathrm{NaCl}, 1.5 \mathrm{mM} \mathrm{Na}_{3} \mathrm{VO}_{4}, 10 \mathrm{mM} \beta$-glycerophosphate, $0.5 \% \mathrm{NP}-40$ (Nonyl 
phenoxypolyethoxylethanol), $\mathrm{pH} 7.4$ ] with $0.5 \mathrm{mM}$ DTT and $10 \mu \mathrm{l} / \mathrm{ml}$ protease inhibitor (Bioshop Canada, Canada Inc. Burlington, ON) cocktail added immediately before use. Homogenates were centrifuged with at $12,000 \mathrm{xg}$ for $20 \mathrm{~min}$ at $4{ }^{\circ} \mathrm{C}$. Supernatants were collected as soluble protein lysate. Protein lysates were then pre-cleared with protein AAgarose beads (Cat\# P2545, Sigma-Aldrich) for $2 \mathrm{~h}$ at $4{ }^{\circ} \mathrm{C}$ followed by centrifuging at $500 \mathrm{xg}$ for $5 \mathrm{~min}$. Supernatant was collected as pre-cleared soluble total protein samples. The protein concentrations of the pre-cleared sample were measured by Bradford assay and then adjusted to $2 \mu \mathrm{g} / \mu 1 \mathrm{using}$ the same homogenizing buffer. Then samples were incubated with primary antibody cross-reacting with Raptor at a ratio of $250: 1$ at $4{ }^{\circ} \mathrm{C}$ with mild rotating overnight. Protein A-Agarose beads were then used to pull down the protein-antibody complexes by incubating with the samples at $4{ }^{\circ} \mathrm{C}$ with mild rotating for $5 \mathrm{~h}$. After precipitation, samples were centrifuged at $500 \mathrm{xg}$ for $5 \mathrm{~min}$, pellets were collected as precipitated products after several rounds of washing with a washing buffer (50 mM Tris $\mathrm{HCl}, 150 \mathrm{mM} \mathrm{NaCl}, 0.05 \%$ Triton X). Same volume of 2X SDS buffer mentioned above was then added to each sample to reach a final protein concentration of $1 \mu \mathrm{g} / \mu \mathrm{l}$ before boiling for $5 \mathrm{~min}$. Immunoblotting was then utilized to analyze the presence of P-mTOR Ser-2448 in the precipitated products of each experimental condition. The same anti-Raptor antibody used for pulling down mTORC1 complex was also used in immunoblotting to verify the result of immunoprecipitation. An antibody specific to SAMD3 $(53 \mathrm{kDa})$ [homologs of both the Drosophila protein, mothers against decapentaplegic (MAD) and the Caenorhabditis elegans protein SMA (from gene sma for small body size)] was used as a non-specific negative control for immunoprecipitation experiment. 
Total RNA isolation (Chapter 4\&6)

Total RNA samples were isolated from all experimental groups using Trizol RNA extraction method. All solutions and materials were treated with $0.1 \% \mathrm{v} / \mathrm{v}$ diethylpyrocarbonate (DEPC) before use. Samples of muscle and liver ( $\sim 100 \mathrm{mg})$ from each experimental group were homogenized in $1 \mathrm{ml}$ Trizol solution (BioShop Canada Inc. Burlington, ON) using a Polytron homogenizer. Homogenates were then mixed with $300 \mu \mathrm{l}$ choloroform before centrifugation at $10,000 \mathrm{xg}$ for $15 \mathrm{~min}$ at $4^{\circ} \mathrm{C}$. The upper aqueous layer of each sample was transferred into a new microcentrifuge tube. A $750 \mu 1$ aliquot of 2-propanol was added into each tube to precipitate RNA. After 10 min at room temperature, samples were then centrifuged at $10,000 \mathrm{xg}$ for $10 \mathrm{~min}$ at $4^{\circ} \mathrm{C}$. After removing the supernatant, the total RNA pellet was washed with $70 \%$ ethanol and centrifuged again at $10,000 \mathrm{xg}$ for $10 \mathrm{~min}$ at $4^{\circ} \mathrm{C}$. After removing excess ethanol, pellets were air dried and then re-suspended in $25 \mu \mathrm{l}$ DEPC treated water. RNA concentrations of the samples were determined using a GeneQuant Pro spectrophotometer (Phamacia). The 260/280 value was used to indicate the purity of the RNA samples $\left(\mathrm{A}_{260 / 280}\right.$ ratio $=$ 1.8-2). All the samples were then standardized to a final concentration of $1 \mu \mathrm{g} / \mu \mathrm{l}$ using DEPC treated water. The quality of total RNA samples was checked by $18 \mathrm{~S}$ and $28 \mathrm{~S}$ ribosomal RNA (rRNA) bands using electrophoresis on a 1\% agarose gel. 
Gene specific RT-PCR (Reverse transcriptase polymerase chain reaction) primers were designed using DNAMAN 4.0 and PrimerDesigner 3.0 programs. Primers were designed based on the consensus region for the cDNA sequence from multiple sequenced vertebrates. The housekeeping gene $\beta$-actin was used for standardization. All the primers used in the thesis are listed in Table 2.2.

Aliquots of total RNA samples containing $5 \mu$ RNA were incubated with $1 \mu 1$ of $200 \mathrm{ng} / \mu 1$ Oligo dT in a thermal cycler (Eppendorf, ON, Canada) for $5 \mathrm{~min}$ at $65^{\circ} \mathrm{C}$. After chilling on ice for $1 \mathrm{~min}$, the reactions were subject to first strand cDNA synthesis using the following reagents: $4 \mu \mathrm{l} 5 \mathrm{x}$ first strand buffer, $2 \mu 1100$ mM DTT (Dithiothreitol), $1 \mu \mathrm{l}$ $25 \mathrm{mM}$ dNTP (Deoxynucleotide triphosphate) mixture and $1 \mu \mathrm{M}$ M-MLV reverse transcriptase enzyme (all from Invitrogen). Reactions were incubated at $42^{\circ} \mathrm{C}$ for $45 \mathrm{~min}$. The resulting cDNA were then diluted and used for PCR amplification of gstal and $\beta$ actin genes. Each PCR reaction contains $10.6 \mu 1$ double distilled sterile water, $4 \mu \mathrm{l}$ diluted cDNA sample, $1 \mu \mathrm{l}$ primer mixture (final concentration of $1.5 \mu \mathrm{M}$ for both forward and reverse primers), $1.2 \mu 150 \mathrm{mM} \mathrm{MgCl}_{2}, 0.4 \mu \mathrm{l} 25 \mathrm{mM}$ dNTP mixture, $0.8 \mu \mathrm{l} \mathrm{Taq}$ polymerase and $2 \mu 1$ 10X PCR buffer (Invitrogen). Reactions were programmed as follows: $7 \mathrm{~min}$ at $94^{\circ} \mathrm{C}$ prior to 35 cycles of $1 \mathrm{~min}$ at $94^{\circ} \mathrm{C}, 1 \mathrm{~min}$ at an annealing temperature specific to individual genes (see table 2.2 for details) and finally $1 \mathrm{~min}$ at $72^{\circ} \mathrm{C}$, followed by $10 \mathrm{~min}$ at $72^{\circ} \mathrm{C}$ for a final elongation. PCR products were then separated using gel electrophoresis on a $1.5 \%$ agarose gel with 1 X TAE buffer $(40 \mathrm{mM}$ Tris base, $0.1 \%$ acetic acid and $1 \mathrm{mM}$ EDTA, $\mathrm{pH} 8.5$ ).

miRNA stemloop amplification (Chapter 4) 
For analysis of levels of selected miRNA species and 5s rRNA, a stemloop based RT-PCR was carried out (Biggar et al., 2009). Briefly, for each reaction, a $5 \mu \mathrm{L}$ sample containing $5 \mu \mathrm{g}$ of wood frog total RNA was incubated with $1 \mu \mathrm{L}$ of $250 \mathrm{mM}$ miRNAspecific stem-loop primer for 5 min at $95^{\circ} \mathrm{C}$ for RNA denaturation and followed by 5 min at $60^{\circ} \mathrm{C}$ for stem-loop annealing. The reactions were then chilled on ice for $1 \mathrm{~min}$ and then to each the following reagents were added: $4 \mu \mathrm{L} 5 \mathrm{x}$ first strand buffer, $2 \mu \mathrm{L} 100 \mathrm{mM}$ DTT, $1 \mu \mathrm{L} 25 \mathrm{mM}$ dNTP mixture and $1 \mu \mathrm{L}$ M-MLV reverse transcriptase (all from Invitrogen). Reverse transcription reactions were carried out for $30 \mathrm{~min}$ at $16^{\circ} \mathrm{C}$ followed by $40 \mathrm{~min}$ at $42^{\circ} \mathrm{C}$ and $85^{\circ} \mathrm{C}$ for $5 \mathrm{~min}$. The resulting product was diluted $\left(10^{-1}\right.$ to $\left.10^{-3}\right)$ and used for PCR. Each PCR reaction was prepared with $10.2 \mu \mathrm{L}$ double distilled sterile water, $4 \mu \mathrm{L}$ diluted RT product, $1.6 \mu \mathrm{L}$ primer mixture $(25 \mu \mathrm{M}$ for both forward and reverse), $1 \mu \mathrm{L} 50 \mathrm{mM} \mathrm{MgCl} 2,0.4 \mu \mathrm{L} 25 \mathrm{mM}$ dNTP mixture, $0.8 \mu \mathrm{L}$ Taq polymerase and $2 \mu \mathrm{L}$ 10x PCR buffer (Invitrogen). The PCR program was $10 \mathrm{sec}$ at $95^{\circ} \mathrm{C}$ prior to 35 cycles of $15 \mathrm{sec}$ at $95^{\circ} \mathrm{C}$ and $1 \mathrm{~min}$ at $60^{\circ} \mathrm{C}$; this was followed by a final hold at $4{ }^{\circ} \mathrm{C} .5 \mathrm{~s}$ RNA was used as a reference for quantification. All stemloop primers used are listed in Table 2.2.

Nuclear Extracts (Chapter 4\&6)

Samples of frozen muscle and liver were quickly homogenized 1:2 w:v using a Douce homogenizer in homogenization buffer (10 mM HEPES pH 7.9, $10 \mathrm{mM} \mathrm{KCl,} 10$ mM EDTA) with $10 \mu 1 / \mathrm{ml} 100 \mathrm{mM}$ DTT stock and $10 \mu 1 / \mathrm{ml}$ Protease Inhibitor Cocktail (BioShop Canada Inc. Burlington, ON) added immediately prior to homogenization. Homogenates were centrifuged at $10,000 \mathrm{xg}$ for $10 \mathrm{~min}$ at $4^{\circ} \mathrm{C}$ and supernatants were 
collected as cytoplasmic fractions. Pellets were re-suspended in an extraction buffer containing 20 mM HEPES pH 7.9, $400 \mathrm{mM} \mathrm{NaCl}, 1 \mathrm{mM}$ EDTA, 10\% v/v glycerol with $10 \mu \mathrm{l} / \mathrm{ml} 100 \mathrm{mM}$ DTT and 10 $\mu \mathrm{l} / \mathrm{ml}$ Protease Inhibitor Cocktail (BioShop Canada Inc. Burlington, ON). Samples were incubated for 60 min on ice on a rocking platform and then centrifuged at $10,000 \mathrm{xg}$ for $10 \mathrm{~min}$ at $4^{\circ} \mathrm{C}$. Supernatants were collected as nuclear extracts. Protein concentrations were determined using the Bradford assay and normalized to $10 \mu \mathrm{g} / \mu \mathrm{l}$. Aliquots of the samples were mixed with equal amount of $2 \mathrm{X}$ SDS buffer and subjected to SDS-PAGE (SDS- Polyacrylamide gel electrophoresis) and immunoblotting as described earlier. An antibody detecting histone $\mathrm{H} 3$ (a nuclear marker) was used to confirm the quality of the nuclear extracts. Samples were stored at $40^{\circ} \mathrm{C}$ until use.

Electrophoretic Mobility Shift Assay (EMSA) (Chapter 6)

EMSA was used to validate the oligonucleotide probe used for transcription factor analysis. Biotin-labeled oligonucleotides containing the ARE (shown in bold underline) were used to generate double stranded probes that were purchased from SIGMA,

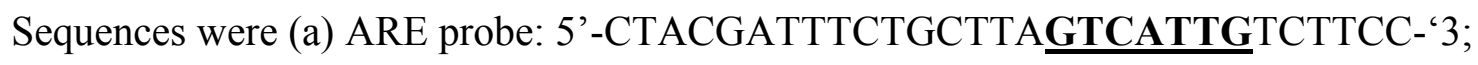
and reverse complimentary (RC): 5'-GGAAGACAATGACTAAGCAGAAATCGTAG$\left.3^{\prime}\right)$. Equal amounts of biotin-labelled and RC probes ( 250 pmole) were mixed and incubated at $94^{\circ} \mathrm{C}$ in a thermal cycler. Oligonucleotide mixtures were then held in the thermal cycler for 20 min to cool down to room temperature in order for biotin-labeled and $\mathrm{RC}$ oligonucleotides to form the double stranded probe. The resulting probe was stored at $-40^{\circ} \mathrm{C}$ for future use. 
For EMSA, aliquots of the $250 \mathrm{pmole} / \mu 1$ stock probe were diluted to $10 \mathrm{ng} / \mu 1$. Nuclear extracts containing $10 \mu \mathrm{g}$ protein were mixed with $1 \mu \mathrm{g}$ poly d(I-C), $2 \mu 1$ EMSA 5X binding buffer (50 mM Tris pH 7.8, $250 \mathrm{mM} \mathrm{NaCl}, 5 \mathrm{mM}$ EDTA, 25\% v:v glycerol, $2.5 \mathrm{mM}$ DTT), and $5 \mu 1$ nuclease-free water. All samples as well as negative controls (no probe, no protein) were allowed to incubate at room temperature for $5 \mathrm{~min}$. After incubation, $1 \mu \mathrm{l}$ probe was added into each reaction and all were incubated at $15^{\circ} \mathrm{C}$ in a thermal cycler for $30 \mathrm{~min}$. Samples (10 $\mu 1$ mixed with $1 \mu \mathrm{l}$ DNA loading dye) were then loaded into wells of a $6 \%$ non-denaturing polyacrylamide gel containing $1 \mathrm{ml}$ pre-chilled 10X TBE (0.89 M Tris base, 0.89 M boric acid, 20 mM EDTA pH 8.0), $4 \mathrm{ml} \mathrm{30 \%}$ acrylamide, $625 \mu 180 \%$ glycerol, $300 \mu 1$ 10\% APS, and $20 \mu 1$ TEMED after a pre-run step (without samples) of $10 \mathrm{~min}$ at $120 \mathrm{~V}$ at $4^{\circ} \mathrm{C}$ ). Samples were separated on the gel at $120 \mathrm{~V}$ for $45 \mathrm{~min}$ at $4^{\circ} \mathrm{C}$. The protein-probe mixtures were electroblotted onto a presoaked nylon membrane (Pall Corporation, NY, USA) using wet transfer in 0.5X TBE. After transfer, the probes were crosslinked to the membrane by placing the membrane in an oven at $80^{\circ} \mathrm{C}$. The membrane was incubated with blocking buffer (Cat\#: CS 7535; Panomics, CA, USA) for 15 min at room temperature. Blocking buffer was then removed, the membrane was washed with washing buffer (Cat\#: CS 7537; Panomics, CA, USA), and then $5 \mu 1$ of diluted streptavidin-HRP mixture (Cat\#: APA012P.1; Bioshop, ON, Canada) was added and incubated for $15 \mathrm{~min}$ at room temperature. Then the membrane was washed again with washing buffer and visualized with enhanced chemiluminescence $\left(\mathrm{H}_{2} \mathrm{O}_{2}\right.$ and Luminol).

Transcription factor binding assay (Chapter 6) 
A ELISA (Enzyme-linked immunosorbent assay) -type transcription factor binding assay was used to assess Nrf2-DNA binding activity using the $250 \mathrm{pmole} / \mu \mathrm{l}$ stock double-stranded oligonucleotide probe described above. The assay was performed on 96-well microplates pre-coated with streptavidin (R\&D Systems, ON, Canada). Aliquots of 250 pmole/ $\mu$ l stock probe were diluted in PBS (phosphate-buffered saline) buffer (1.37 M NaCl, $2.7 \mathrm{mM} \mathrm{KCl}, 10 \mathrm{mM} \mathrm{Na}_{2} \mathrm{HPO}_{4}, 2 \mathrm{mM} \mathrm{KH}_{2} \mathrm{PO}_{4}, \mathrm{pH}$ 7.4) to a final concentration of $0.8 \mathrm{pmole} / \mu \mathrm{l}$. To coat wells with DNA probe, $50 \mu 1$ of $0.8 \mathrm{pmole} / \mu \mathrm{l}$ probe solution was added into each well and incubated for $1 \mathrm{~h}$ at room temperature. After incubation, excessive probe solution was removed and wells were washed with PBST (PBS buffer with $0.1 \%$ Tween 20 added). Aliquots of nuclear extracts containing $8 \mu \mathrm{g}$ protein were added into wells with $0.25 \mu 1$ 100mM DTT, $0.1 \mu \mathrm{l} 20 \mu \mathrm{g} / \mu \mathrm{l}$ salmon sperm DNA (Cat\#: DNA999.5; Bioshop, ON, Canada) and $50 \mu$ l binding buffer (10 mM Hepes, $50 \mathrm{mM} \mathrm{KCl}, 0.5 \mathrm{mM}$ EDTA, $40 \mathrm{mM} \mathrm{NaCl}, 3 \mathrm{mM} \mathrm{MgCl} 2,10 \%$ glycerol, $0.5 \mathrm{mg} / \mathrm{ml}$ bovine serum albumin and $0.05 \% \mathrm{NP}-40, \mathrm{pH} 7.9$ ). After $1 \mathrm{~h}$ incubation with mild shaking on a rocking platform at room temperature, solutions were removed from the wells, followed by washing with PBST. A $60 \mu 1$ aliquot of diluted primary antibody specific to Nrf2 (1:1000 dilution, in PBST) was added into each sample well and allowed to incubate on a mild shaking platform at room temperature for $1 \mathrm{~h}$. After primary antibody incubation, wells were washed with PBST and incubated with diluted anti-rabbit IgG secondary antibody (1:4000 dilution in PBST) on a shaker at room temperature for $1 \mathrm{~h}$. Wells were washed extensively with PBST after incubation with secondary antibody and then $60 \mu 1$ TMB (Tetramethylbenzidine) solution was added into each well and allowed to incubate for 15 min avoiding light. Once the incubation was done, $60 \mu 1$ of stop 
solution $(1 \mathrm{M} \mathrm{HCl})$ was added into each well. The plate was then read on a microplate reader at $450 \mathrm{~nm}$. A test run with no probe, no protein and no primary controls was performed prior to quantification runs and no probe controls were always included in all quantification runs.

The effects of glucose on Nrf2 DNA binding activity were assessed in vitro. Aliquots of a glucose stock solution (5 M, diluted in PBS) were added into assay wells with the nuclear extracts from control frogs to give final concentrations of $200 \mathrm{mM}$ or $500 \mathrm{mM}$. The rest of the assay was performed as described above.

Antioxidant capacity assay (Chapter 6)

An antioxidant assay kit (Cat\# 709001; Cayman Chemicals, Michigan, USA) was used for assessing antioxidant capacity. Samples were prepared in a homogenization buffer according to the manufacturer's guide and the assay was performed following the instruction manual. Briefly, for each reaction, $10 \mu \mathrm{l}$ diluted extracts of muscle and liver of wood frogs were added into wells of a 96 well microplate with $10 \mu 1$ of metmyoglobin and $150 \mu \mathrm{l}$ chromogen. Reactions were initiated with addition of $40 \mu \mathrm{l}$ of hydrogen peroxide working solution and the plate was placed on a shaker at room temperature for 5 min. After incubation, the plate was read at $750 \mathrm{~nm}$ using a microplate reader.

Multiplex assay (Chapter 6)

A Luminex kit (Cat\# H0XSTMAG; Millipore, MA, USA) was used to examine protein levels of catalase, SOD1, SOD2, PRX2 and TRX12, in liver and muscle of 
control, $24 \mathrm{~h}$ frozen, $8 \mathrm{~h}$ thawed, $2 \mathrm{~h}$ saline-loaded and $2 \mathrm{~h}$ glucose-loaded frogs. Sample preparation followed the specific protocol for tissue samples provided with the kit. Briefly, up to $50 \mathrm{mg}$ samples of frozen tissue were weighed and homogenized 1:5 w:v in a homogenization buffer (Millipore, MA USA, with protease inhibitors cocktail added, Bioshop, ON, Canada). After a 15 min incubation at $4^{\circ} \mathrm{C}$, homogenates were centrifuged at $10,000 \mathrm{xg}$ for $10 \mathrm{~min}$ at $4^{\circ} \mathrm{C}$. Supernatants were collected as total soluble protein samples. Protein concentrations were determined by Bradford assay and subsequently diluted to $0.75 \mu \mathrm{g} / \mu 1$ using the assay buffer provided with the kit. The assay was then performed following the user manual. A Magpix instrument (Millipore, MA, USA) with Milliplex xPONENT software (Millipore, Billerica, MA) was used to collect the data.

\section{Statistics (All chapters)}

Band densities on immunoblots or agarose gels were visualized using the ChemiGenius imaging system (Syngene, Frederick, MD) and the associated GeneTools software. Immunoblot band intensities were standardized against a group of three Coomassie blue stained bands in the same lane that were constant across all lanes and distant from the protein of interest, as previously described (Krivoruchko and Storey, 2010a; Zhang et al., 2013). Sybr Green stained agarose gels were visualized under UV light. The bands used for quantification were from the most dilute cDNA samples that showed visible images in order to ensure that band densities were not saturating. All PCR bands were standardized against corresponding $\beta$-actin or $5 \mathrm{~s}$ rRNA bands amplified from the same RNA samples. All data from freeze-thaw cycles were assessed using one-way analysis of variance followed by a Tukey test. The Student's t-test was used for 
comparing data saline-loaded and glucose-loaded frogs. In all cases, $\mathrm{P}<0.05$ was accepted as showing significant differences between groups.

\section{Contribution to the thesis}

All experimental procedures presented in Chapter 2 (Material and methods) were done by the author with the exception of 1 . collecting frogs from the field, 2. tetracycline bath, and 3. moss preparation. All animal experimental conditions and parameters (e.g. time point, freezing temperatures, dehydration and anoxia levels) were determined based on the animal work done previously in the lab. 
Table 2.1 Antibody list

\begin{tabular}{|c|c|c|c|}
\hline Gene & Company & Cat \# & $\begin{array}{l}\text { Secondary } \\
\text { Antibody }\end{array}$ \\
\hline Cdc2 & Genscript & A01295 & Anti-Rabbit \\
\hline Cdk2 & Santa Cruz & sc-748 & Anti-Rabbit \\
\hline Cdk4 & Abcam & ab6553 & Anti-Rabbit \\
\hline Cdk6 & Abcam & ab6553 & Anti-Rabbit \\
\hline p-Cdk T14/Y15 & Santa Cruz biotechnology & sc-28435 & Anti-Rabbit \\
\hline Cyclin A & Abcam & ab6552 & Anti-Rabbit \\
\hline Cyclin B1 & Abcam & ab6552 & Anti-Rabbit \\
\hline Cyclin D1 & Abcam & ab6552 & Anti-Rabbit \\
\hline Cyclin E & Abcam & ab6552 & Anti-Rabbit \\
\hline $\mathrm{p}-\mathrm{Cdc} 25 \mathrm{a}$ S76 & Genscript & A00439 & Anti-Rabbit \\
\hline p-Cdc25c S216 & Genscript & A00420 & Anti-Rabbit \\
\hline p-p21 T145 & Genscript & A01160 & Anti-Rabbit \\
\hline p-p27 T187 & Genscript & A00333 & Anti-Rabbit \\
\hline Akt & Genscript & A00301 & Anti-Rabbit \\
\hline p-Akt S473 & Genscript & A00272 & Anti-Rabbit \\
\hline p-Akt T308 & Genscript & A00275 & Anti-Rabbit \\
\hline PDK-1 & Genscript & A00208 & Anti-Rabbit \\
\hline p-PDK01 S241 & Genscript & A00207 & Anti-Rabbit \\
\hline PTEN & Genscript & A00302 & Anti-Rabbit \\
\hline FOXO1 & Cell Signaling & 2880 & Anti-Rabbit \\
\hline p-FOXO1 S256 & Genscript & A00395 & Anti-Rabbit \\
\hline mTOR & Genscript & A01154 & Anti-Rabbit \\
\hline p-mTOR S2448 & Genscript & A00643 & Anti-Rabbit \\
\hline Raptor & Cell Signaling & 2280 & Anti-Rabbit \\
\hline p-Raptor S863 & Abgent & AP3495a & Anti-Rabbit \\
\hline GßL & Cell Signaling & $3274 \mathrm{~S}$ & Anti-Rabbit \\
\hline p-TSC2 S939 & Abgent & AP3415a & Anti-Rabbit \\
\hline p-PRAS40 T246 & Santa Cruz biotechnology & sc-32629 & Anti-Rabbit \\
\hline 4EBP & Genscript & A00328 & Anti-Rabbit \\
\hline p-4EBP T45 & Genscript & A00328 & Anti-Rabbit \\
\hline S6K & Genscript & A00516 & Anti-Rabbit \\
\hline p-S6K T389 & Santa Cruz biotechnology & sc-11759 & Anti-Rabbit \\
\hline SMAD3 & Cell signaling & $9523 \mathrm{P}$ & Anti-Rabbit \\
\hline Nrf2 & Santa Cruz biotechnology & sc-722 & Anti-Rabbit \\
\hline GSK3 $\beta$ & Genscript & A00196 & Anti-Rabbit \\
\hline p-GSK3 $\beta$ S9 & Santa Cruz biotechnology & sc-11757 & Anti-Goat \\
\hline
\end{tabular}


Table. 2.2 Primer list

\begin{tabular}{|c|c|c|c|}
\hline Gene & $\begin{array}{l}\text { Annealing } \\
\text { temperature } \\
\left({ }^{\circ} \mathrm{C}\right)\end{array}$ & Primer & Primer Sequence \\
\hline \multirow[t]{2}{*}{ pten } & 60.8 & Forward & 5'-CARGAGGATGGATTCGACTT-3' \\
\hline & & Reverse & 5'-CTCTGGTCCKTACTTCYCCA-3' \\
\hline \multirow[t]{2}{*}{$\beta$-actin } & 53.8 & Forward & 5'-AGAAGTCGTGCCAGGCATCA-3' \\
\hline & & Reverse & 5'-AGGAGGAAGCTATCCGTGTT-3' \\
\hline \multirow[t]{2}{*}{ GSTal } & 60.8 & Forward & 5'-GAAGYTGGTGCAGACCAGAG-3' \\
\hline & & Reverse & 5'-CCAGGCTGMAGRAACTTCTT-3' \\
\hline \multirow[t]{4}{*}{$5 s$ rRNA } & 60 & Stem loop & 5 '- \\
\hline & & & CTCACAGTACGTTGGTATCCTTGTGATGTTCGATGCC \\
\hline & & Forward & $\begin{array}{l}\text { ATATTGTACTGTGAGAAAGCCTA-3' } \\
\text { 5'-CTCGTCTGATCTCGGAAGCT-3' }\end{array}$ \\
\hline & & Reverse & 5'-CTCACAGTACGTTGGTATCCTTGTG-3' \\
\hline \multirow[t]{4}{*}{$m i R-26 a$} & 60 & Stem loop & 5 '- \\
\hline & & RT & $\begin{array}{l}\text { CTCACAGTACGTTGGTATCCTTGTGATGTTCGATGCC } \\
\text { ATATTGTACTGTGAGGCCTATCC-3, }\end{array}$ \\
\hline & & Forward & 5'-ACACTCCAGCTGGGTTCAAGTAATCCAGG-3' \\
\hline & & Reverse & 5'-CTCACAGTACGTTGGTATCCTTGTG-3' \\
\hline \multirow[t]{4}{*}{$m i R-126$} & 60 & Stem loop & 5 '- \\
\hline & & RT & $\begin{array}{l}\text { CTCACAGTACGTTGGTATCCTTGTGATGTTCGATGCC } \\
\text { ATATTGTACTGTGAGGCGCATT-3, }\end{array}$ \\
\hline & & Forward & 5'-ACACTCCAGCTGGGTCGTACCGTGAGTAA-3' \\
\hline & & Reverse & 5'-CTCACAGTACGTTGGTATCCTTGTG-3' \\
\hline \multirow{4}{*}{$m i R-217$} & 60 & Stem loop & $5 '-$ \\
\hline & & & $\begin{array}{l}\text { CTCACAGTACGTTGGTATCCTTGTGATGTTCGATGCC } \\
\text { ATATTGTACTGTGAGATCCAATC-3' }\end{array}$ \\
\hline & & Forward & 5'-ACACTCCCAGCTGGGTACTGCATCAGGAAC-3' \\
\hline & & Reverse & 5'-CTCACAGTACGTTGGTATCCTTGTG-3' \\
\hline
\end{tabular}


Fig. 2.1

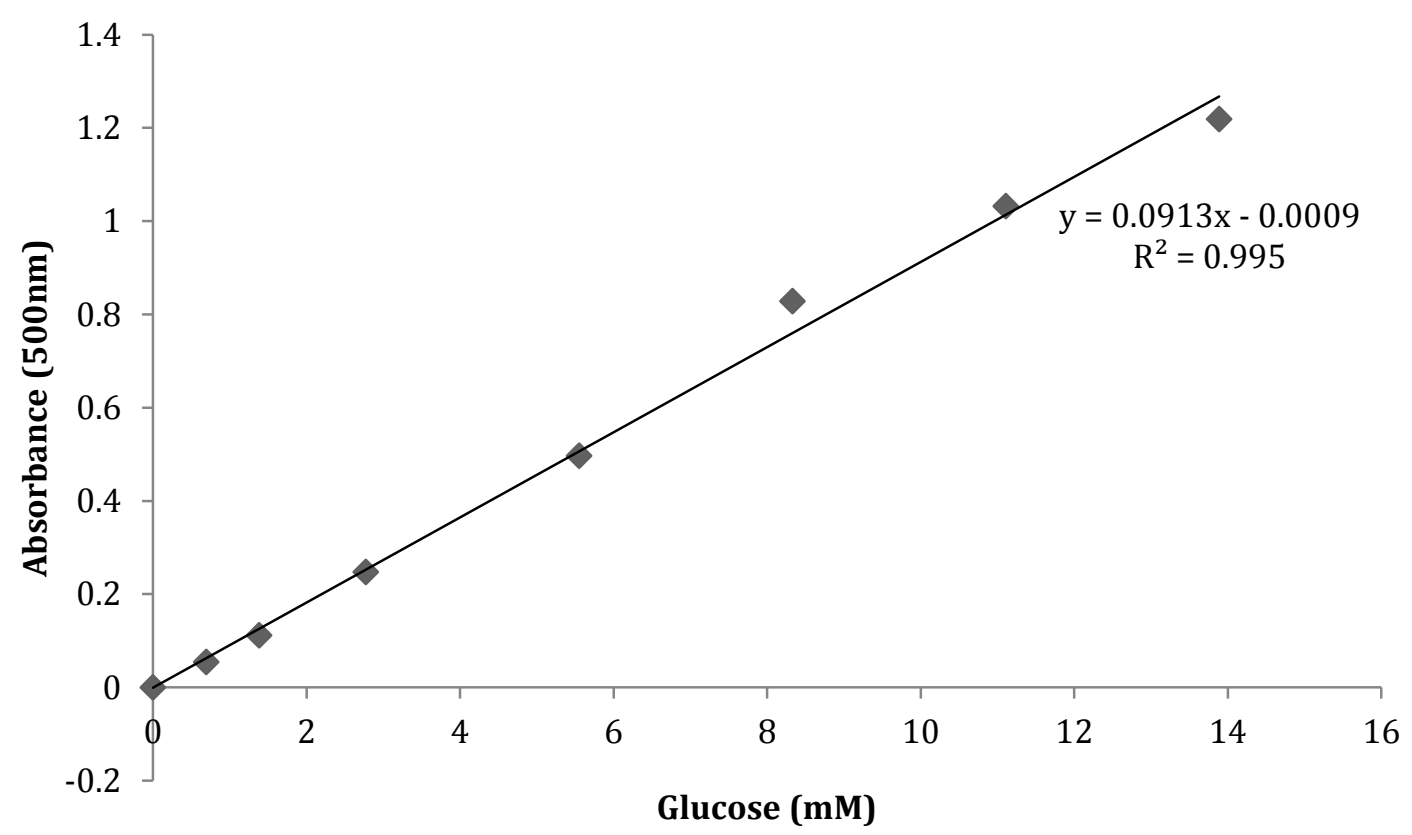

Fig. 2.1. Glucose assay kit standard curve monitored at $500 \mathrm{~nm}$. Values have been corrected by subtraction of blanks 


\section{Chapter 3 Cell cycle regulation}




\section{Introduction}

As described in Chapter 1, long term survival in the hypometabolic frozen state is aided by strongly suppressing optional ATP-consuming processes. Thus, I hypothesized that the energy-expensive cell cycle would also be a critical target for suppression when wood frogs freeze. The current chapter explores the status of cell cycle components in wood frog liver in response to freezing.

Cell cycle progression depends on cyclins and Cdks that assemble reversibly at different cell cycle stages to form Cdk-cyclin complexes (Biggar and Storey, 2009). Complexes of particular Cdk-cyclin combinations are only present during their corresponding functional phases under controls from cell signaling networks and through posttranslational modifications, such as phosphorylation (Kaldis, 1999; Lolli and

Johnson, 2005). In higher eukaryotes, more than $10 \mathrm{Cdks}$ have been discovered; among these, Cdc 2, Cdk 2, 4 and 6 are four essential Cdks directly involved in regulating the progression of different phases as well as phase transitions. When triggered by signaling systems, the cell cycle starts with the formation of type D cyclin-Cdk complexes. Cyclin $\mathrm{D}$ (with isoforms D1, D2 and D3) interacts with Cdk 4 and 6 during $\mathrm{G}$ phase resulting in Cdk-mediated phosphorylation of Rb (retinoblastoma) family members, including Rb, p107 and p130. Unphosphorylated Rb forms a complex with E2F (E2 promoter binding factor) that suppresses the activity of this transcription factor. The inhibitory effect of $\mathrm{Rb}$ on $\mathrm{E} 2 \mathrm{~F}$ is relieved by hyperphosphorylation of $\mathrm{Rb}$ by cyclin $\mathrm{D}-\mathrm{Cdk} 4 / 6$ complexes resulting in the dissociation of $\mathrm{Rb}$ proteins from the Rb-E2F complex (Yang et al., 1999). Active E2F then promotes transcription of type A and E cyclins that are used in later stages of cell cycle progression. Cdk 2 forms a complex with cyclin E to promote the 
G1/S phase transition and interacts with type A cyclins throughout S phase to promote DNA synthesis. In addition, the cyclin E-Cdk2 complex also phosphorylates Rb to further activate E2F, forming a positive feedback loop with the transcription factor (Fig. 3.1B). Then Cdc 2 combines with type A cyclins to initiate S/G2 phase transition. Subsequently, Cdc 2 interacts with cyclin B to promote M progression (Draetta et al., 1989). Cdks are regulated by phosphorylation at Thr-14 and Tyr-15 in higher eukaryotic systems; these covalent modifications lead to conformational changes that disturb the ATP phosphatebinding orientations, causing inhibitory effects on kinase activity (

et al., 2004). Inhibitory phosphorylation of Cdks has been proposed as one of the timing mechanisms that control the activation or deactivation of specific Cdkcyclin pairs at each stage of the cell cycle (Lew and Kornbluth, 1996). For example, in higher eukaryotes, both Thr-14 and Tyr-15 sites on Cdc2 are dephosphorylated at the end of G2 phase but are kept phosphorylated at the stages prior to M phase, where Cdc 2cyclin B activity is not required (Lew and Kornbluth, 1996). Phosphorylation at these sites also contributes to stress responsive cell cycle arrest in multiple species (Booher $\boldsymbol{e t}$ al., 1997; Yuan et al., 2004; De Schutter et al., 2007) (Fig. 3.1).

Because a critical step in Cdk activation is dephosphorylation at Thr-14/Tyr-15, the phosphatases that catalyze this $(\mathrm{Cdc} 25 \mathrm{a}, \mathrm{Cdc} 25 \mathrm{c})$ are also closely regulated and are targets of kinase signaling pathways (Iliakis et al., 2003; Manke et al., 2005). Cdc25a acts primarily at G1/S transitions in normal cell cycle progression, whereas Cdc $25 \mathrm{c}$ regulates G2/M phase transition (Jinno et al., 1994; Karlsson-Rosenthal and Millar, 2006) (Fig. 3.1A). In addition, Cdc25 phosphatases play important roles in linking stressinduced kinase cell signaling to cell proliferation. Specifically, Cdc25a and Cdc25c are 
phosphorylated by Checkpoint kinase $(\mathrm{ChK})$ in response to ATM/ATR signaling and p38 MAPK also phosphorylates Cdc25 proteins (Bulavin et al., 2002; Iliakis et al., 2003). Cdc25a can be phosphorylated on multiple sites, including Ser-76, which is critical in destabilizing the protein. Phosphorylation on Ser-76 of Cdc25a by Chk1 leads to subsequent phosphorylation events on Ser-82 and Ser-88 that, in turn, create an ubiquitin ligase recognition motif on the protein to stimulate ubiquitin-dependent degradation (Busino et al., 2003; Jin et al., 2003). In the case of Cdc25c, phosphorylation on Ser-216 inhibits the protein by regulating its subcellular distribution. Chk phosphorylates the site, leading to binding of Cdc25c to 14-3-3 protein, which prevents the protein from relocalizing into the nucleus (Manke et al., 2005).

CdkIs are another major group of proteins that regulate Cdk-cyclin complex formation and activity. Two key members of the CIP/KIP family of CdkIs are p21 and p27, which play critical roles in regulating Cdk-Cylin formation; other CdkIs include the INK4 family (Sherr and Roberts, 1999). Different from the CdkIs of the INK4 (Inhibitor of cyclin-dependent kinase 4) family, which only interact with Cdks, CIP/KIP members regulate both Cdk and cyclin proteins (Sherr and Roberts, 1999). p21 and p27 are able to interact with all major Cdk-cyclin complexes involved in cell cycle progression, and their function shifts depending upon the target. It is widely accepted that p21 inhibits cell cycle progression by interfering with formation of Cdk 2-cyclin complexes involved in $\mathrm{G}$ and $\mathrm{S}$ phases and by blocking PCNA (proliferating cell 1 nuclear antigen)-dependent DNA synthesis (Harper et al., 1993; Waga et al., 1994). On the other hand, p27 interferes with ATP binding of the S-phase Cdk 2-cyclin A complex, thereby inhibiting its kinase activity.27 However, emerging evidence also suggests that 
both p 21 and p27 contribute to D-type Cdk-cyclin complex formation (Soos et al., 1996; Labaer et al., 1997; Russo et al., 1999; Cheng et al., 1999). Furthermore, CIP/KIP CdkIs facilitate the inhibitory effects of INK4 CdkIs (Sherr and Roberts, 1999). p21 and p27 are both subject to phosphorylation-dependent regulation. Phosphorylation on Thr-145 of p21 by Akt (protein kinase B), which responds to insulin signaling, prevents the protein from being transported into the nucleus (Zhou et al., 2001). In the case of p27, the protein can be phosphorylated on Thr-187 by Cdk 2-cyclin E, triggering a ubiquitin-mediated protein degradation pathway (Montagnoli et al., 1999).

Overall, then, Cdks and cyclins along with Cdc25 phosphatases and CIP/KIP CdkIs are essential regulators of cell cycle progression. The present study analyzed the effects of whole-body freezing and thawing on the protein expression and phosphorylation patterns of these key cell cycle components and regulators in wood frog liver (a proliferating tissue). In addition, the effects of anoxia and dehydration, two component stresses of whole-body freezing, were also assessed along with recovery from these stresses (reoxygenation and rehydration). Anoxia is imposed due to cessation of breathing/circulation in the frozen animal, and cell dehydration/shrinkage that arises because water is drawn out of cells to join growing extracellular ice masses (Storey, 2004c; Storey and Storey, 2004a). The present work expands an initial limited analysis of the effects of anoxia and dehydration alone on selected cell cycle components in wood frog liver and skeletal muscle (Roufayel et al., 2011) and demonstrates that reversible cell cycle suppression is an integral component of the survival of freezing, anoxia and dehydration in wood frogs. 


\section{Results}

Expression of Cdk proteins in wood frog liver in response to freezing, anoxia and dehydration

Relative protein levels of Cdks as well as the overall phosphorylation of Cdks at Thr-14/Tyr-15 were assessed by immunoblotting in liver of control $\left(5^{\circ} \mathrm{C}\right.$ acclimated), 24 $\mathrm{h}$ frozen $\left(\right.$ at $\left.-2.5^{\circ} \mathrm{C}\right), 24 \mathrm{~h}$ anoxic $\left(\right.$ at $\left.5^{\circ} \mathrm{C}\right)$ and $40 \%$ dehydrated $\left(\right.$ at $\left.5^{\circ} \mathrm{C}\right)$ frogs as well as under the corresponding recovery conditions: $8 \mathrm{~h}$ thawed, $4 \mathrm{~h}$ aerobic recovery and rehydration, respectively. The polyclonal antibodies used cross-reacted with single bands at the known molecular weights of the individual Cdks in other animals. Expression of Cdks showed a stress specific pattern. Figure 3.2 shows that after $24 \mathrm{~h}$ of freezing exposure, the relative protein levels of $\mathrm{Cdc} 2, \mathrm{Cdk} 4$ and Cdk 6 dropped significantly in wood frog liver; levels were $66 \pm 6 \%, 61 \pm 3 \%$ and $73 \pm 5 \%$ of the corresponding control values, respectively. However, levels of these three proteins all returned to control values after $8 \mathrm{~h}$ thawing. By contrast, no significant change was observed for liver Cdk 2 protein levels during freezing, but levels increased significantly by $1.3 \pm 0.05$-fold when frogs thawed. The overall phosphorylation state of Cdks (Thr-14/Tyr-15) was unchanged during freezing, but phospho-Cdk content increased significantly in frog liver by $1.4 \pm$ 0.09-fold during thawing as compared with the control group (Fig. 3.2).

Total protein levels of all four Cdk proteins decreased significantly in wood frog liver in response to a $24 \mathrm{~h}$ anoxia exposure, as compared with the normoxic controls (Fig. 3.3). Levels decreased to just $37 \pm 9,44 \pm 1,39 \pm 4$ and $41 \pm 2 \%$ of the corresponding control values for $\mathrm{Cdc} 2, \mathrm{Cdk} 2, \mathrm{Cdk} 4$ and $\mathrm{Cdk} 6$, respectively. A similar anoxic suppression of Cdk protein levels in liver to $47-65 \%$ of aerobic values was reported 
previously by Roufayel et al. (2011). During aerobic recovery, the levels of all four Cdks rebounded to the normoxic control level. By contrast, the overall phosphorylation state of Cdks at Thr14/Tyr15 increased strongly in frog liver after $24 \mathrm{~h}$ anoxia, rising by $2.7 \pm$ 0.38-fold, but again returning to control levels after $4 \mathrm{~h}$ of aerobic recovery.

Figure 3.4 shows the effects of dehydration and rehydration on Cdks in wood frog liver. Levels of all four Cdks decreased significantly in liver of frogs dehydrated to $40 \%$ of total body water lost; relative levels were just $44 \pm 2,34 \pm 5,52 \pm 3$ and $45 \pm 12 \%$ for $\mathrm{Cdc} 2, \mathrm{Cdk} 2, \mathrm{Cdk} 4$ and $\mathrm{Cdk} 6$, respectively, in liver of dehydrated frogs as compared with control animals. After rehydration, levels of all Cdks increased again although Cdk 2 protein levels remained significantly below the control value $(64 \pm 1 \%)$ whereas Cdk 4 increased to $1.48 \pm 0.07$-fold higher than the corresponding control. Dehydration also altered the total content of phospho-Cdk, which rose by $1.22 \pm 0.02$-fold during dehydration but returned to control values after rehydration.

Expression of cyclin proteins in wood frog liver in response to freezing, anoxia and dehydration.

Relative protein levels of cyclins were assessed via immunoblotting in liver from control, $24 \mathrm{~h}$ frozen, $24 \mathrm{~h}$ anoxic and $40 \%$ dehydrated wood frogs along with the corresponding recovery conditions. Polyclonal antibodies cross-reacted with protein bands at molecular weights corresponding to the known values for cyclins in other animals. Figure 3.5 shows the effect of freeze/thaw on cyclin levels in wood frog liver. In response to $24 \mathrm{~h}$ freezing, relative protein levels of cyclin A and B1 decreased significantly to $73 \pm 5$ and $55 \pm 7 \%$ of the corresponding control values and remained low 
after $8 \mathrm{~h}$ thawing (at $74 \pm 7$ and $58 \pm 4 \%$ of controls, respectively). However, cyclin D1 showed an opposite response, increasing by $1.74 \pm 0.11$-fold in liver of $24 \mathrm{~h}$ frozen frogs and rising further to $3.16 \pm 0.29$-fold higher than control after $8 \mathrm{~h}$ thawed. Cyclin E levels did not change during freezing but decreased significantly during thawing to a value just $49 \pm 3 \%$ of the control.

Amounts of all four cyclins decreased significantly in wood frog liver responding to anoxia stress (Fig. 3.6). Cyclin levels in liver of $24 \mathrm{~h}$ anoxic frogs fell to $79 \pm 3,60 \pm$ 3, $47 \pm 7$ and $69 \pm 3 \%$ for cyclins A, B1, D1 and E, respectively, as compared with corresponding controls. Similar effects of anoxia on liver cyclin levels were reported previously; levels decreased to 50-75\% of control values (Roufayel et al., 2011). After 4 $\mathrm{h}$ of aerobic recovery, levels of cyclin A returned to control values, and cyclin B1 levels rose somewhat (to $74 \pm 3$ of controls), but cyclin D1 and E remained suppressed (50 \pm 5 and $63 \pm 7 \%$ of controls).

Dehydration to $40 \%$ of total body water lost also resulted in significant decreases in the levels of all cyclins in liver (Fig. 3.7). Levels of cyclins A, B1, D1 and E decreased to $73 \pm 7,52 \pm 12,24 \pm 4$ and $59 \pm 2 \%$ of the corresponding control values, respectively, in response to dehydration. However, upon rehydration back to full water content, levels of cyclin A and B1 rebounded to control values. Cyclin D1 levels also increased by 3fold but were still significantly lower than controls $(67 \pm 4 \%)$. Only cyclin E showed no recovery after rehydration; levels remained at $67 \pm 3 \%$ of control values.

Expression of phospho-Cdc25 proteins in wood frog liver in response to freezing, anoxia and dehydration. 
Cdc25a and Cdc25c were evaluated by analyzing relative changes in the phosphorylation state of the proteins. Phospho-Cdc25a (Ser-76) and phospho-Cdc25c (Ser-216) levels were examined in wood frog liver responding to freezing, anoxia, dehydration stresses and corresponding recovery conditions (Fig. 3.8). In response to 24 $\mathrm{h}$ freezing, relative phosphorylation of Cdc25a (Ser-76) dropped to $75 \pm 5 \%$ of the control value but increased strongly after $8 \mathrm{~h}$ thawing, rising to $1.59 \pm 0.02$-fold higher than controls (Fig. 3.8A). Phospho-Cdc25c (Ser-216) levels in liver were unchanged by freezing but increased strongly after freezing to $1.69 \pm 0.2$-fold higher than control values. Figure $3.8 \mathrm{~B}$ shows the responses to $24 \mathrm{~h}$ anoxia exposure and $4 \mathrm{~h}$ of aerobic recovery. Phospho-Cdc25a (Ser-76) levels increased strongly, with a fold change of $3.2 \pm$ 0.03 in liver after $24 \mathrm{~h}$ anoxia exposure, but returned to aerobic control values after $4 \mathrm{~h}$ of aerobic recovery. Similarly, phospho-Cdc25c (Ser-216) levels increased greatly under anoxia $(3.79 \pm 0.2$-fold increase). Subsequently, levels fell by about one-half after $4 \mathrm{~h}$ recovery but remained $1.99 \pm 0.03$-fold higher than the control values. Dehydration to $40 \%$ of total body water lost and subsequent rehydration resulted in a similar pattern of responses to those seen for anoxia/recovery. Figure $3.8 \mathrm{C}$ shows that the relative content of phospho-Cdc25a (Ser-76) increased strongly by $6.2 \pm 0.5$-fold in frog liver after $40 \%$ dehydration but returned to control values after rehydration. Phospho-Cdc25c (Ser-216) also increased by $4.6 \pm 0.4$-fold in liver from $40 \%$ dehydrated frogs and again fell back to control levels after rehydration.

Expression of phospho-CdkI proteins in wood frog liver in response to freezing, anoxia and dehydration. 
Changes in the relative phosphorylation states of two CdkIs, phospho-p21 (Thr145) and phospho-p27 (Thr-187), were also assessed in liver from control, $24 \mathrm{~h}$ frozen, $24 \mathrm{~h}$ anoxic and $40 \%$ dehydrated wood frogs, along with the corresponding recovery conditions (Fig. 3.9). During freezing, phospho-p21 (Thr-145) content increased by 1.77 \pm 0.2 -fold, but after $8 \mathrm{~h}$ of thawing, values returned to control levels (Fig. 3.9A). Levels of phospho-p27 (Thr-187) showed a very strong $3.53 \pm 0.1$-fold increase after $24 \mathrm{~h}$ freezing but again returned to control values after $8 \mathrm{~h}$ thawing. The patterns of phosphorylation of these proteins were very different in response to anoxia. Figure 3.9B shows that the relative phosphorylation of p21 (Thr-145) decreased under anoxia to $62 \pm$ $4 \%$ of the control value and remained low after $4 \mathrm{~h}$ of aerobic recovery $(46 \pm 9 \%$ of control values). By contrast, the phosphorylation state of $\mathrm{p} 27$ was unchanged by anoxia exposure and aerobic recovery. Dehydration to $40 \%$ of total body water lost led to a significant decrease in both phosphorylated p21 (Thr-145) and p27 (Thr-187) contents (Fig. 3.9C). Phospho-p21 (Thr-145) levels in liver of dehydrated frogs dropped to $74 \pm$ $2 \%$ of control values and remained low after rehydration (64 $\pm 6 \%$ as compared with controls). Phospho-p27 (Thr187) content showed the same pattern. Levels decreased significantly to $56 \pm 1 \%$ of controls after $40 \%$ dehydration and again remained low after rehydration ( $62 \pm 2 \%$ as compared with controls). 


\section{Discussion}

Survival of freezing by wood frogs involves two main strategies. First, to maximize the survival time of cells and organs under the ischemic, dehydrated conditions of the frozen state, energy expenditures are suppressed by shutting down various ATPexpensive metabolic processes. Second, cryoprotective defenses are put in place to protect cells, including upregulation of ice-nucleating proteins, selected novel proteins and production of high levels of the cryoprotectant, glucose (Storey and Storey, 2004a). Liver has the major role in orchestrating cryoprotective defenses for the whole animal, particularly in the synthesis of the huge amounts of glucose that are exported and then taken up by all other organs (Storey, 1987a; Storey and Storey, 1994). Therefore, it is not a surprise that wood frog liver is one of the last organs in the frog to freeze (and also one of the first to thaw) (Rubinsky et al, 1994). Multiple aspects of liver responses to freezing have been explored on both physiological and biochemical levels (Storey and Storey, 1984, 1996; Storey, 1987a; Storey et al., 1992; Conlon et al., 1998; Holden and Storey, 2000; Cowan and Storey, 2001). One of the most energy-expensive activities of cells is cell division, and studies in a variety of systems have documented cell cycle arrest in response to multiple stresses (e.g., nutrient or oxygen limitation) and also as an integral part of natural hypometabolism (hibernation, diapause, etc.) (Bilodeau et al,. 2000; Pietenpol and Stewart, 2002; Carey et al., 2003; De Schutter et al., 2007; Imadome et al., 2008; Perucca et al., 2009; Valcourt et al., 2012). Therefore, the status of cell cycle activity can be considered as one of the signature cellular events that reflects both energetics and stress responses in cells and organs (Biggar and Storey, 2009). 
The present study analyzed the responses of multiple core cell cycle components to the stresses of freezing, anoxia and dehydration in wood frog liver and also assessed recovery after the stress was removed. Generally, the results showed that levels of most of Cdk and cyclin proteins were suppressed in response to each of these stresses but typically returned to control or near control levels during recovery (Figs. 3.2-3.7). The findings are generally consistent with cell cycle suppression and/or arrest in response to freezing, anoxia or dehydration stresses. However, stress- and protein-specific regulatory patterns emerged. For example, $24 \mathrm{~h}$ anoxia exposure and $40 \%$ dehydration generally triggered stronger suppression of both Cdk and cyclin protein levels than did $24 \mathrm{~h}$ freezing. Cdk protein levels dropped to $40-50 \%$ of control levels in liver of anoxic and dehydrated frogs, but during freezing, Cdc 2, Cdk 4 and Cdk 6 decreased to $60-70 \%$ of control values, whereas Cdk 2 levels were unaffected. Furthermore, phosphorylation of Cdks at Thr14 and Tyr15 increased strongly in response to anoxia and dehydration, indicating a reduced activity state of Cdks under these stress conditions. However, phosphorylation status did not change in response to freezing and actually increased during thawing, whereas total $\mathrm{Cdk}$ phosphorylation returned to control values during recovery from anoxia or dehydration (Figs. 3.2-3.4).

General responses by cyclins to stress were similar to those of Cdks. Levels of all cyclins assessed decreased substantially in response to anoxia and dehydration, as did Cyclin A and B1 in liver of $24 \mathrm{~h}$ frozen frogs (Figs. 3.5-3.7). However, Cyclin E levels did not change during freezing, and cyclin D1 levels increased by 1.7-fold. Because cyclin D1 and cyclin E are responsible for G1 phase and G1/S transition, respectively, the results suggest that freezing may have a lesser inhibitory impact on protein expression of 
the factors involved in entry stages of the cell cycle. It is worth noting that $24 \mathrm{~h}$ freezing exposure failed to change protein expression levels of both Cdk 2 and cyclin E. Since the main function of the Cdk 2-cyclin E complex is to promote G1/S transition and initiate DNA synthesis, freezing exposure might not affect the progress of this stage. However, suppressed levels of both Cdk 4 and Cdk 6 (Fig. 3.2) suggest that lower amounts of functional Cdk 4/6-cyclin D1 complexes could form, which may be sufficient to block progression of G1 phase. These particular arrest patterns might be a specific response to freezing (or low temperature) in wood frog liver, since both anoxia and dehydration stresses triggered strong reductions in Cdk 2 and cyclin E levels. These results for freezing effects are similar to a pattern of cell cycle arrest that occurred in response to cold shock in mammalian cell cultures (from non-hibernating species), where cold exposure (cooling from $37^{\circ} \mathrm{C}$ to $16-20^{\circ} \mathrm{C}$ ) blocked $\mathrm{G} 2 / \mathrm{M}$ transition but not progression through $\mathrm{G}$ or S phases (Rieder and Cole, 2002). The authors suggested that at least one pathway/element that is present during G phase is selectively disrupted below a critical temperature (Rieder and Cole, 2002). In the current study, the temperature change between control $\left(5^{\circ} \mathrm{C}\right)$ and frozen $\left(-2.5^{\circ} \mathrm{C}\right)$ conditions is not large but may nonetheless have an impact on the cyclin/Cdk patterns in liver of frozen frogs as compared with the patterns seen in response to anoxia or dehydration (done at constant $5^{\circ} \mathrm{C}$ ). Furthermore, although still speculative at this time, such a temperature-induced block may also be significant to overall cell cycle suppression, if cyclin D has a novel function to play during freeze tolerance.

Cyclin D1 protein levels increased strongly in frog liver after $24 \mathrm{~h}$ freezing, although they were suppressed under anoxia or dehydration exposure. Since levels of 
Cdk 4 and 6 (partners of cyclin D) were suppressed during freezing, it is proposed that the freeze-specific enhancement of cyclin D1 alone may not be related to cell cycle control. Indeed, a recent study has shown that cyclin D1 can bind to the promoter region of the notch1 gene to promote its transcription, suggesting that cyclin D1 has an additional function as a transcription factor (Bienvenu et al., 2010). The notch1 gene encodes the Notch1 transmembrane receptor, which is a core component in the Notch signaling pathway. This pathway can promote hepatic glucose production by upregulating the gene for glucose-6-phosphatase (G6PC) in both FOXO1 (forkhead box O type1)-dependent and -independent manners (Pajvani et al., 2011). This could be significant for wood frogs because of their unique cryoprotection strategy that requires the synthesis and accumulation of extremely large amounts of glucose as the cryoprotectant; e.g., liver and plasma levels rise to $200-300 \mathrm{mM}$ as compared with $\sim 5$ $\mathrm{mM}$ in controls, and amounts in all other organs soar as they take up glucose from the blood (Storey, 1987a, 2004c; Storey and Storey, 2004a). Virtually all of the glucose cryoprotectant is synthesized by liver and depends on glucose-6-phosphatase as the terminal step in converting glucose-6-phosphate (derived from glycogen) to glucose for export. Triggering of this massive glucose output has previously been linked with (1) $\beta$ adrenergic signaling, which stimulates a large increase in liver glycogen phosphorylase activity within minutes when freezing begins on the skin surface (Storey and Storey, 1996) and (2) with very high levels of glucose transporters in wood frog hepatocyte plasma membranes as compared with freeze-intolerant frogs (King et al., 1993). However, just as critical to glucose production may be a rapid increase in glucose-6phosphatase activity, an enzyme that resides in the endoplasmic reticulum membrane 
(Storey and Storey, 1984). Unlike freeze-tolerant insects that produce cryoprotectants on a seasonal basis over several weeks, frogs only synthesize glucose in direct response to the initiation of tissue freezing and therefore have only a few hours to flood their tissues with glucose (exported from liver) before the inward movement of the ice front halts all blood circulation (Storey and Storey, 1984, 2004a). An unusual role for cyclin D1 as a transcription factor to activate the Notch signaling pathway and rapidly upregulate g6pc expression in liver might, therefore, be an important component of the overall enhancement of glucose production during freezing.

Upon recovery from freezing, anoxia or dehydration stresses, the data showed that protein levels of most Cdks rose again to control values, suggesting that a recovery of energy metabolism sufficient to allow resumption of protein synthesis had occurred within the liver (Figs. 3.2-3.4). However, levels of most cyclins remained reduced after 8 $\mathrm{h}$ thawing, $4 \mathrm{~h}$ aerobic recovery or rehydration. Given that cyclins are key regulators of Cdks, this suggests that full recovery of highly energy-expensive cell processes, such as cell cycle activity, had still not occurred within the time frame of the recovery conditions used. Furthermore, even though Cdk levels recovered upon thawing, the overall phosphorylation state of Cdks increased in thawed frogs (Fig. 3.2), indicating reduced functionality of Cdks. In addition, the data again indicated a stress specific pattern of response. After $8 \mathrm{~h}$ thawing, cyclin D1 levels were strongly elevated, but expression levels of cyclins that are required for progressing through G1/S transition (cyclin E), S phase (cyclin A), G2 phase (cyclin A), G2/M transition (cyclin A) and M phase (cyclin B1) were all substantially lower than control levels, suggesting continued cell cycle arrest at these stages. The elevated cyclin D1, after $8 \mathrm{~h}$ thawing, might still be related to glucose 
production through Notch signaling. Taken together, the results suggest that cell cycle progression is still inhibited in $8 \mathrm{~h}$ thawed frogs. Indeed, a number of complex physiological behaviors take many hours re-establish after thawing (e.g. skeletal muscle support for posture or jumping) (Layne et al., 1998), and this may also be true of complex energy-expensive metabolic processes.

Except for cyclin A protein levels, $4 \mathrm{~h}$ of aerobic recovery after $24 \mathrm{~h}$ anoxia was also insufficient to re-establish control levels of liver cyclins (Fig. 3.6), although Cdk levels recovered and phospho-Cdk content returned to control values within this time (Fig. 3.3). Thus, these results suggest that although Cdks levels had risen again, activity of the cell cycle as a whole might still be impaired after $4 \mathrm{~h}$ of aerobic recovery. Rehydration after $40 \%$ dehydration resulted in recovery of cyclin A and B1 levels and partial restoration of other cyclins (Fig. 3.7) and Cdks (other than Cdk2) along with Cdk phosphorylation state, suggesting a general restoration of these core cell cycle components once frogs were fully rehydrated. Hence, it can be concluded that none of the three stresses caused permanent damage to the cell cycle capacity of liver, but the pattern and timing of recovery was stress-specific. This is in line with the fact that all three stresses are fully survivable by wood frogs.

Due to the negative effects of phosphorylation on Cdc25 phosphatases, this posttranslational modification is considered to be a marker for regulation of these phosphatases that are responsible for dephosphorylating Cdk proteins during cell cycle progression. Phosphorylation patterns of Cdc25a (Ser-76) and Cdc25c (Ser-216) were stress-specific. Phospho-Cdc25a (Ser-76) levels decreased significantly in liver from $24 \mathrm{~h}$ frozen frogs (by $\sim 25 \%$ ) compared with controls but were strongly elevated in 24-h anoxic 
$(\sim 3$-fold) and $40 \%$ dehydrated frogs ( $\sim 6$-fold) (Fig. 3.8$)$. This indicates that freezing acts positively toward Cdc25a stability, whereas anoxia and dehydration would cause destabilization of the protein as a result of facilitating ubiquitin-mediated proteolysis (Busino et al., 2003; Jin et al., 2003). A similar response was seen for Cdc25c; the phosphorylation state of Cdc25c (Ser-216) did not change during freezing, but phosphoCdc25c (Ser-216) content increased strongly in response to anoxia and dehydration. Thus, freezing may have little effect on the subcellular distribution of $\mathrm{Cdc} 25 \mathrm{c}$, whereas anoxia and dehydration exposures would promote the 14-3-3 protein-mediated exclusion of Cdc25c from the nucleus (Jin et al., 2005). Overall, the data indicate negative effects of anoxia and dehydration on Cdc25 phosphatases. This, in turn, correlates with the enhanced phosphorylation of Cdks that was observed under these conditions and is consistent with cell cycle suppression. Furthermore, the destabilizing effects of these phosphorylations on Cdc25 proteins also fits well with data from a previous study that showed that the total protein content of Cdc25a and Cdc25c decreased significantly in wood frog liver in response to anoxia and dehydration (Roufayel et al., 2011). During recovery from these stresses, the phosphorylation states of Cdc25a and Cdc25c reversed back to near control levels, providing another indicator (in addition to cyclin and Cdk levels) that cell cycle suppression under stress conditions can be readily reversed when the stress is lifted. Interpretation of the response to freezing is more difficult. Based on the phosphorylation patterns, Cdc25 functionality appeared to be elevated somewhat (Cdc25a) or unchanged $(\mathrm{Cdc} 25 \mathrm{c})$ in response to freezing, which is consistent with the unchanged phospho-Cdk content in liver of frozen frogs (Figs. 3.2 and 3.8A). However, during thawing, the phosphorylation state of both proteins rose substantially to 1.5-1.6- 
fold greater than controls (Fig. 3.8A), consistent with the overall increase in phosphorylation of Cdks upon thawing. These data suggest limited posttranslational control of Cdks and Cdc25s during freezing but substantial inhibitory influences on the cell cycle during thawing, at least within the first $8 \mathrm{~h}$, as was also postulated above from the cyclin data.

The data also indicate a stress-specific regulatory pattern for $\mathrm{p} 21$ and $\mathrm{p} 27$. Freezing had negative effects on both CdkI proteins in liver as evidenced by the elevated phosphorylation states of p21 (Thr-145) and p27 (Thr-187) compared with controls (Fig. 3.9A). By contrast, both anoxia and dehydration reduced the phosphorylation state of $\mathrm{p} 21$ (Thr-145) and dehydration also reduced p21 phosphorylation. Phosphorylation of p21 on Thr145 interrupts its import into the nucleus (Zhou et al., 2001), whereas phosphorylation of p27 on Thr187 triggers ubiquitin-mediated breakdown of the protein (Montagnoli et al., 1999). Hence, reduced phosphorylation of these two inhibitor proteins in response to dehydration and of $\mathrm{p} 21$ under anoxia suggests greater inhibition of cyclin-Cdk complexes under stress conditions, which is, again, consistent with cell cycle suppression. With respect to $\mathrm{p} 21$, phosphorylation on Thr-145 is Akt-dependent, and Akt responds to insulin signaling (Zhou et al., 2001). Since Akt signaling in liver plays a key role in glucose metabolism and usually promotes energy-consuming cellular events such as protein synthesis (Ali et al., 2001; Whiteman et al, 2002; Elstrom et al., 2004), this implies that the Akt pathway could be shut down to lower energy expenditures under anoxia or dehydration as part of hypometabolism. Interestingly, p21 phosphorylation state remained suppressed after both aerobic recovery and rehydration, which suggests 
that Akt signaling also remains suppressed, and that the demonstrated recovery of other components of cell cycle regulation, described above, is not under Akt regulation.

Freezing produced opposite responses by p21 and p27 phosphorylation states in liver; phosphoprotein content increased strongly in both cases and reverted to control levels after thawing (Fig. 3.9A). Enhanced phosphorylation of both proteins would generally reduce their inhibitory actions on cyclin-Cdk complex formation by reducing p21 presence in the nucleus and promoting p27 degradation and thereby could promote cell cycle activity (Montagnoli et al., 1999; Zhou et al., 2001). As described previously, p21 binds to Cdk 2 to inhibit G1/S transition but may be required for functional D-type cyclin complex assembly. Therefore, the result may indicate inhibition of G1 but not the G1/S transition of the cell cycle, which agrees with the previous assessment of $\mathrm{Cdk} /$ cyclin responses to freezing. However, another action of p21 may be more important during freezing. Involvement of cytoplasmic p21 in apoptosis inhibition has been reported by Asada et al. (1999), suggesting that the freeze responsive pattern of $\mathrm{p} 21$ regulation in wood frog liver may be involved in preventing stress-induced cell death in the frozen state. This could be one important element of the overall cryoprotection "package" that ensures survival of the extreme stresses imposed on cells and organs by freezing. The strong increase in phospho-p27 (Thr-187) levels during freezing also suggests that p27 may be destabilized. The upstream kinase that phosphorylates p27 is Cdk 2-cyclin E, and neither Cdk 2 nor Cyclin E showed appreciable changes in protein levels during freezing (unlike the decreases in Cdks and cyclins A and B1). Since p27 acts on Cdk 2 and Cdk 4/6 complexes in a similar pattern as p21 (Russo et al., 1996; 
Soos et al., 1996; LaBaer et al., 1997; Cheng et al., 1999), it is not surprising to observe such results.

During thawing, the phosphorylation states of both CdkIs returned to control values, but phospho-p21 levels remained low in liver of both $24 \mathrm{~h}$ anoxic and $40 \%$ dehydrated wood frogs (Fig. 3.9). This suggests that the p21 influence on cell cycle regulation takes a substantially longer time to recover than almost all of the other cell components assessed in the present study. The same appears true for the phospho-p27 response to dehydration, which also remained low in liver after rehydration. This suggests continued inhibition of Cdk 2-cyclin E kinase activity by p27.

In summary, the data indicate that a general inhibition of protein expression of core cell cycle components (cyclins, Cdks) occurs under all three stresses examined (24 h freezing, $24 \mathrm{~h}$ anoxia, $40 \%$ dehydration). This suggests that energy-expensive cell growth and proliferation responses are suppressed under environmental stress conditions that challenge cellular energy-producing capability, and that cell cycle suppression is a component of natural stress-induced hypometabolism. Changes in cyclin and Cdk levels were generally reversed when the stress was lifted, which further supports the idea that cell cycle suppression is a natural survival mechanism employed by stress-tolerant organisms. The patterns of responses by all cell cycle components assessed were essentially the same during stress/recovery for both anoxia and dehydration and were largely consistent with reversible stress-induced cell cycle suppression. However, the response to freezing was more complicated and clearly involves additional influences than those provided by the anoxia and/or dehydration components of the freezing experience. Temperature changes may be one influence, and others may include 
alternative functions for cell cycle components in helping to achieve cryoprotection. For example, cyclin D1 may have an alternative action as a transcription factor that regulates a key gene (encoding glucose-6-phosphatase) involved in cryoprotectant glucose production and an alternate function of $\mathrm{p} 21$ in apoptosis regulation may be important to cell survival in the frozen state. 
Fig. 3.1A

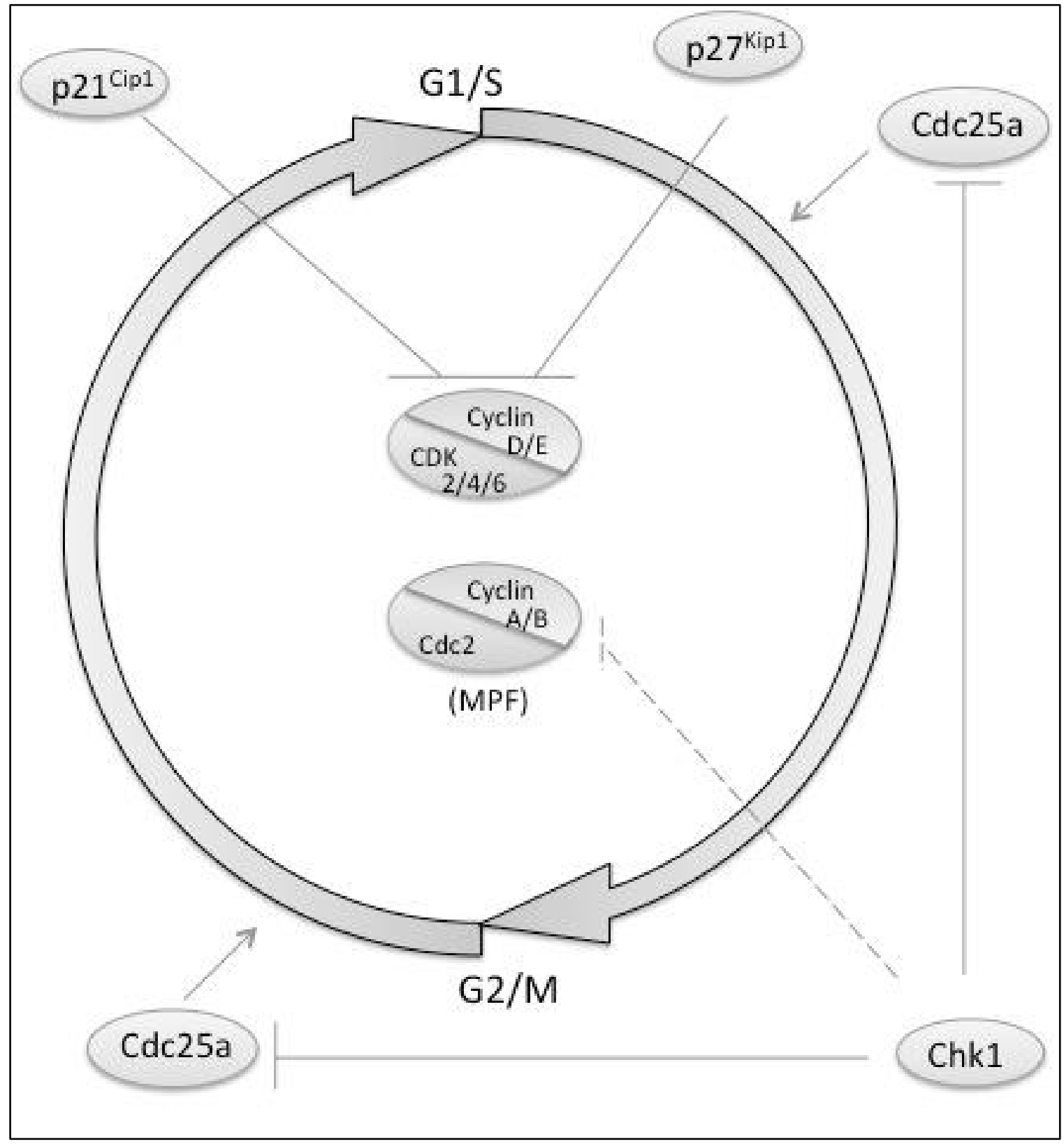


Fig. 3.1B

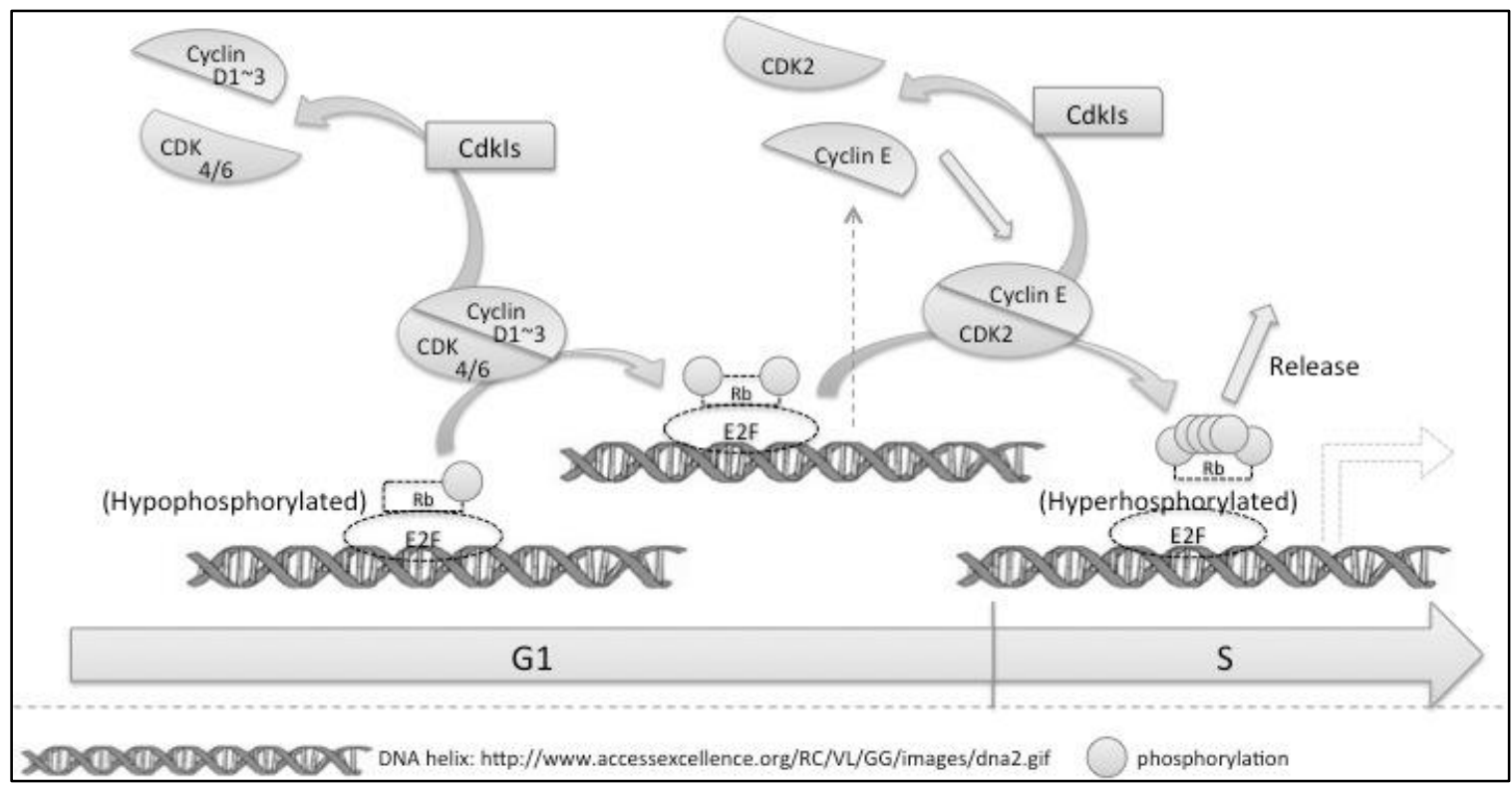

Fig. 3.1 Regulation of cell cycle progression. (A) mechanism of p21, p27, Cdc25a/c and Chk1 in regulation of $\mathrm{Cdk} /$ cyclin complexes; (B) mechanisms of $\mathrm{Rb} / \mathrm{E} 2 \mathrm{~F}$ complex in promoting cell cycle G1/S transition; as well as the phosphorylation regulation from $\mathrm{Cdk} /$ cyclin complexes on $\mathrm{Rb} / \mathrm{E} 2 \mathrm{~F}$ complex in the cell cycle $\mathrm{G} 1 / \mathrm{S}$ progression. 
Fig. 3.2

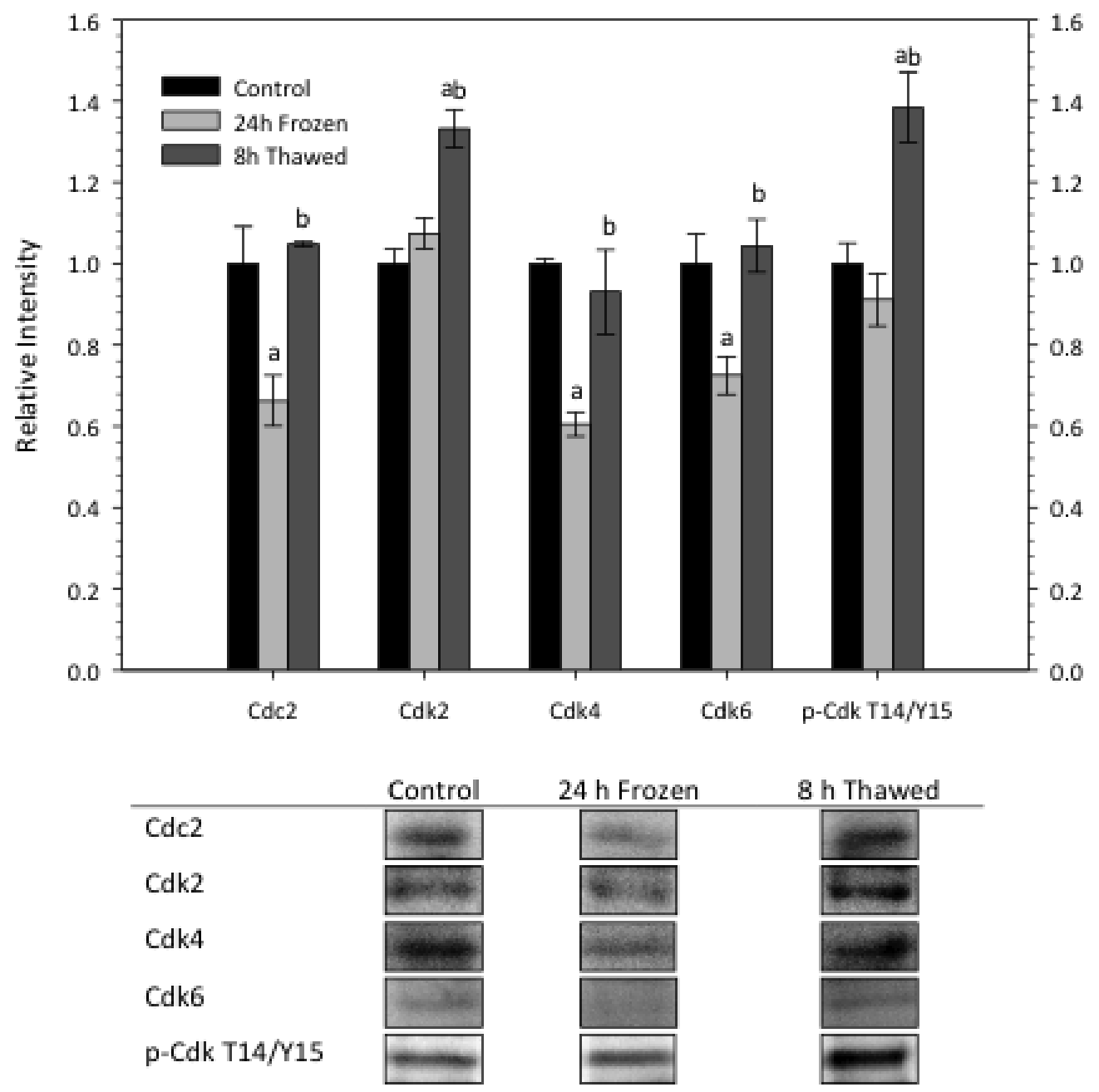

Figure 3.2. Effects of $24 \mathrm{~h}$ freezing and $8 \mathrm{~h}$ thawing on Cdk protein levels as well as total phosphorylation of Cdks on Thr14/Tyr15 (p-Cdk T14/Y15) in wood frog liver. Upper panel shows a histogram of standardized relative protein levels under control, frozen and thawed conditions; data are means \pm SEM, $n=3-4$ independent trials. a - Significantly different from the corresponding control $(p<0.05) ; b$ - Significantly different from the $24 \mathrm{~h}$ frozen value. Lower panel shows representative immunoblot bands from each group. 
Fig. 3.3

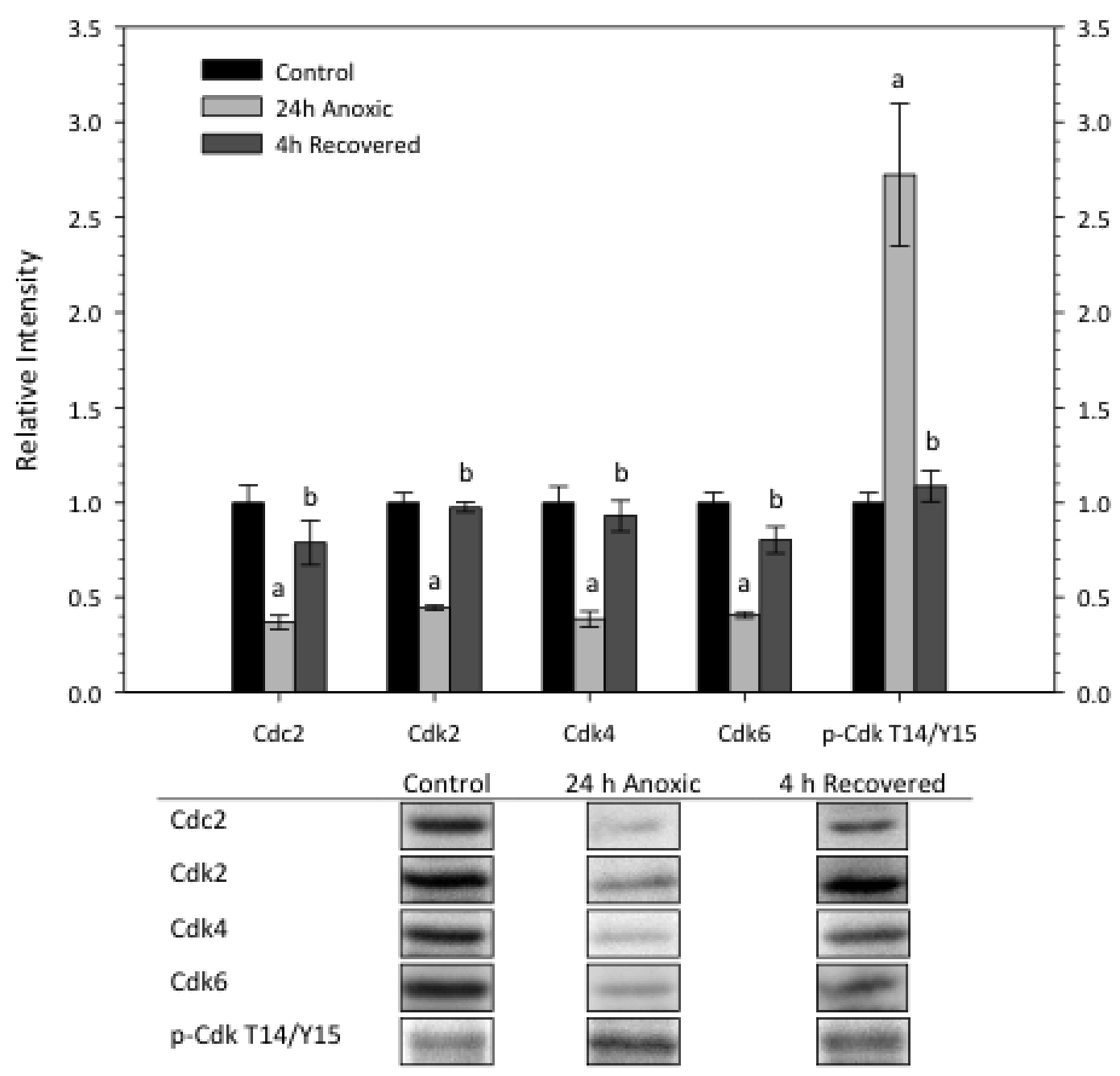

Figure 3.3. Effects of $24 \mathrm{~h}$ anoxia exposure and $4 \mathrm{~h}$ of aerobic recovery on Cdk protein levels as well as total phosphorylated Cdks Thr14/Tyr15 (p-Cdk T14/Y15) in wood frog liver. a - Significantly different from the corresponding control $(\mathrm{p}<0.05)$; $\mathrm{b}$ -

Significantly different from the $24 \mathrm{~h}$ anoxia value. Other information as in Figure 3.2. 
Fig. 3.4

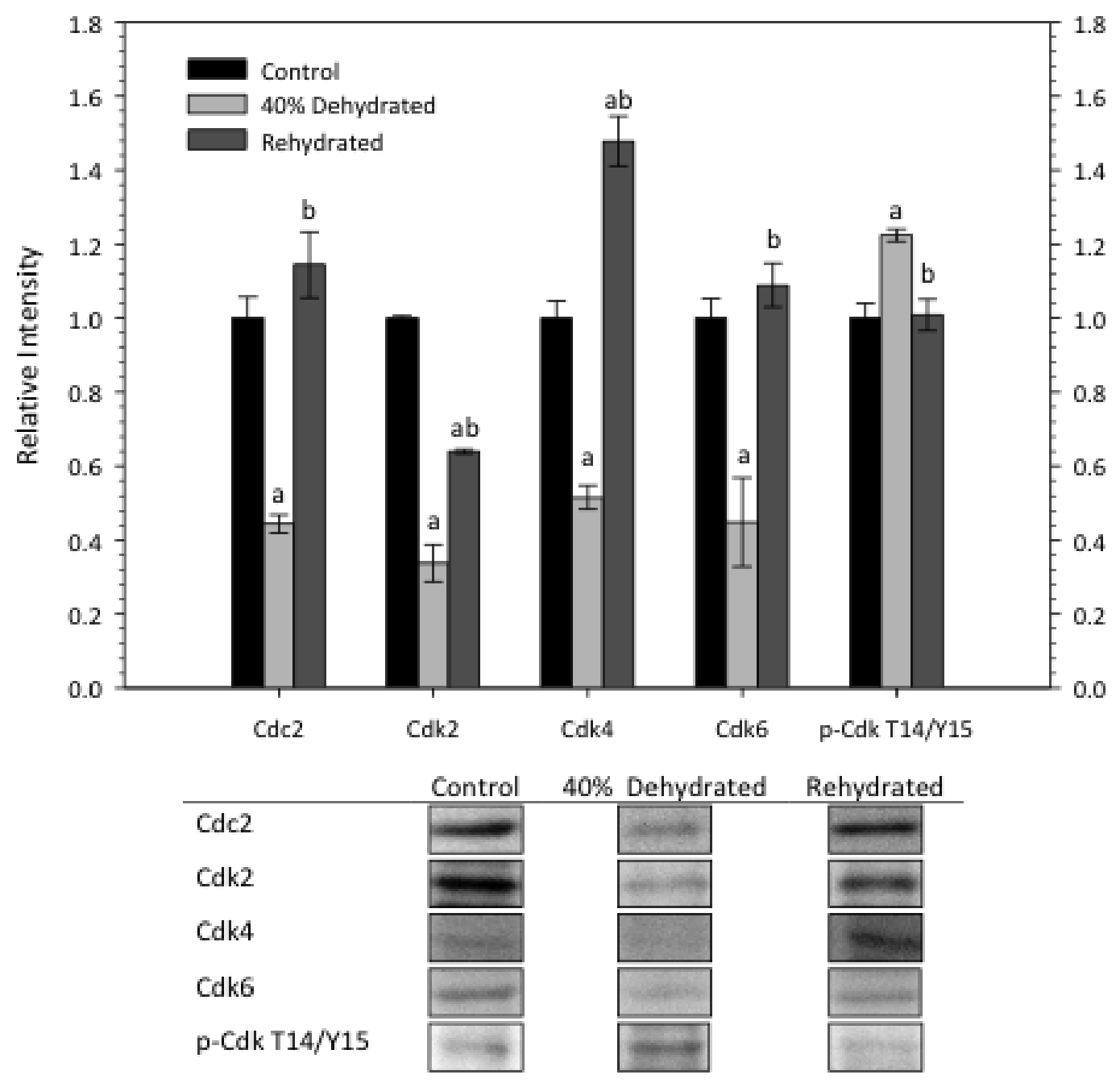

Figure 3.4. Effects of $40 \%$ dehydration and rehydration on protein levels of Cdks as well as total phosphorylated Cdks Thr14/Tyr15 (p-Cdk T14/Y15) in wood frog liver. a Significantly different from the corresponding control $(\mathrm{p}<0.05) ; \mathrm{b}$ - significantly different from the $40 \%$ dehydrated value. Other information as in Figure 3.2. 
Fig. 3.5

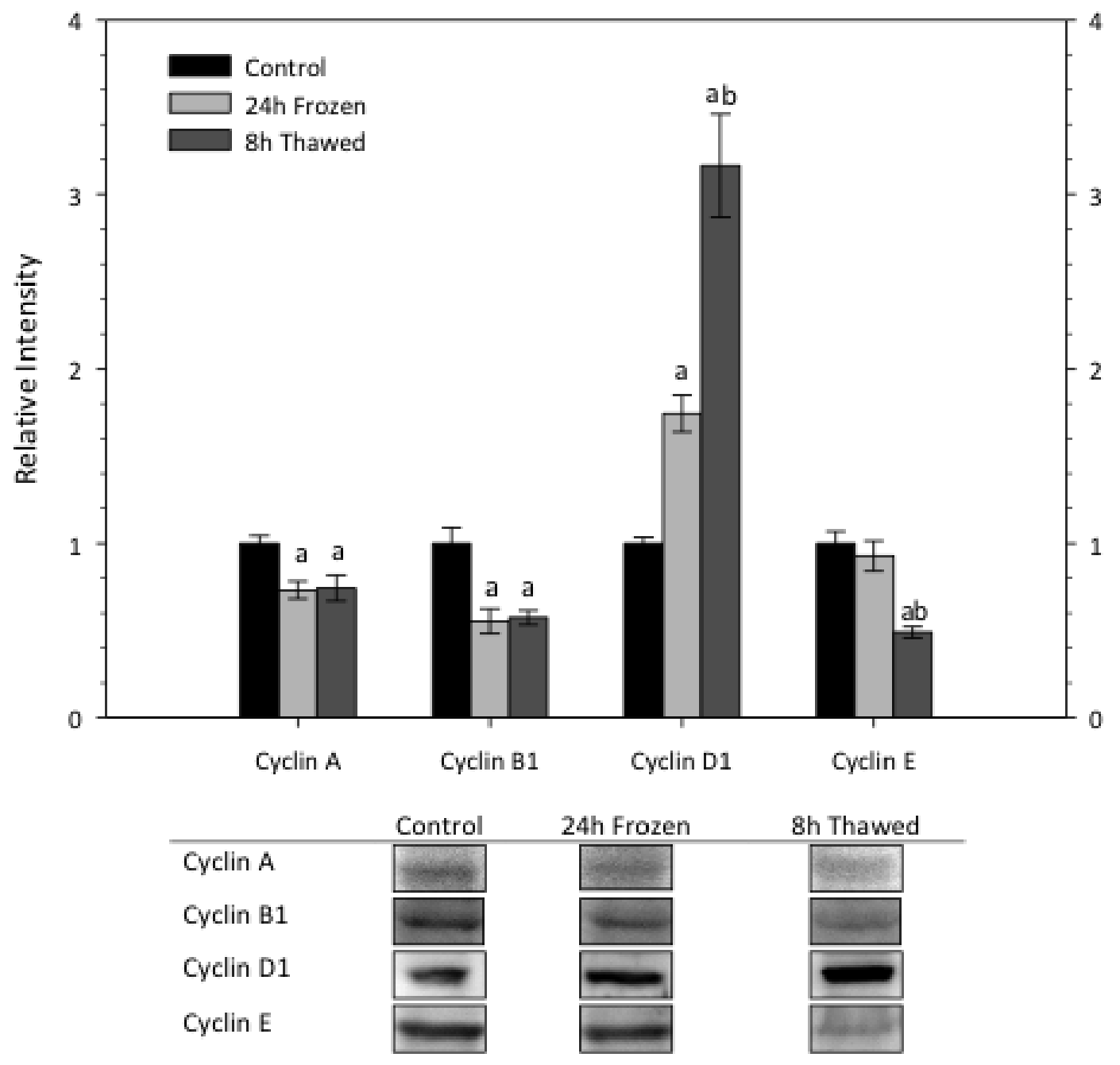

Figure 3.5. Effects of $24 \mathrm{~h}$ freezing and $8 \mathrm{~h}$ thawing on protein levels of cyclins in wood frog liver. Upper panel is the histogram showing standardized relative protein levels under control, frozen and thawed conditions; data are means \pm SEM, $n=3-4$ independent trials. a - Significantly different from the corresponding control $(\mathrm{p}<0.05)$; $\mathrm{b}$ Significantly different from the $24 \mathrm{~h}$ frozen value. Lower panel shows representative immunoblot bands from each group. 
Fig. 3.6

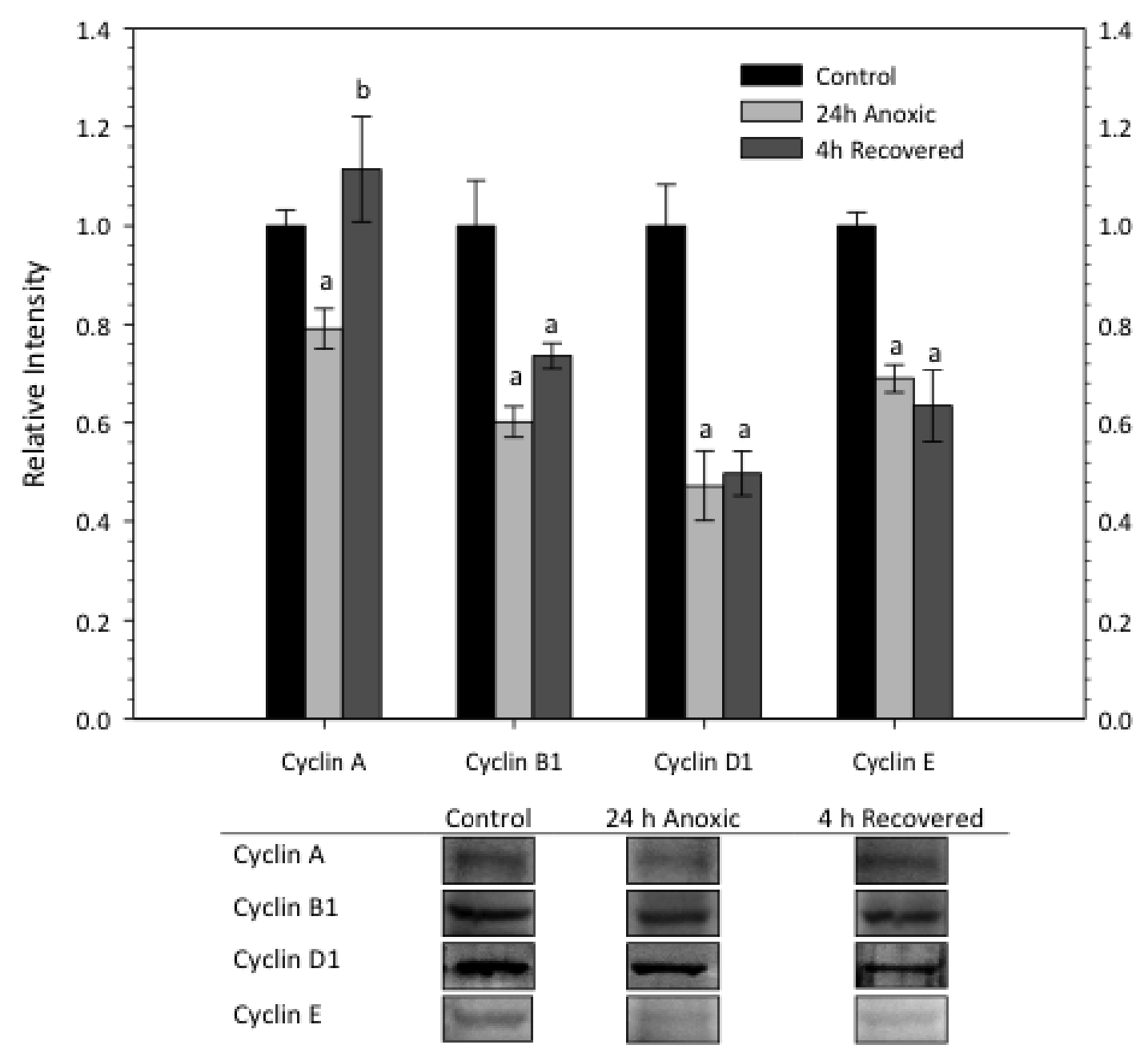

Figure 3.6. Effects of $24 \mathrm{~h}$ anoxia exposure and $4 \mathrm{~h}$ aerobic recovery on protein levels of cyclins in wood frog liver. a - Significantly different from the corresponding control $(p<$ $0.05)$; $\mathrm{b}$ - Significantly different from the $24 \mathrm{~h}$ anoxic value. Other information as in Figure 3.5. 
Fig. 3.7

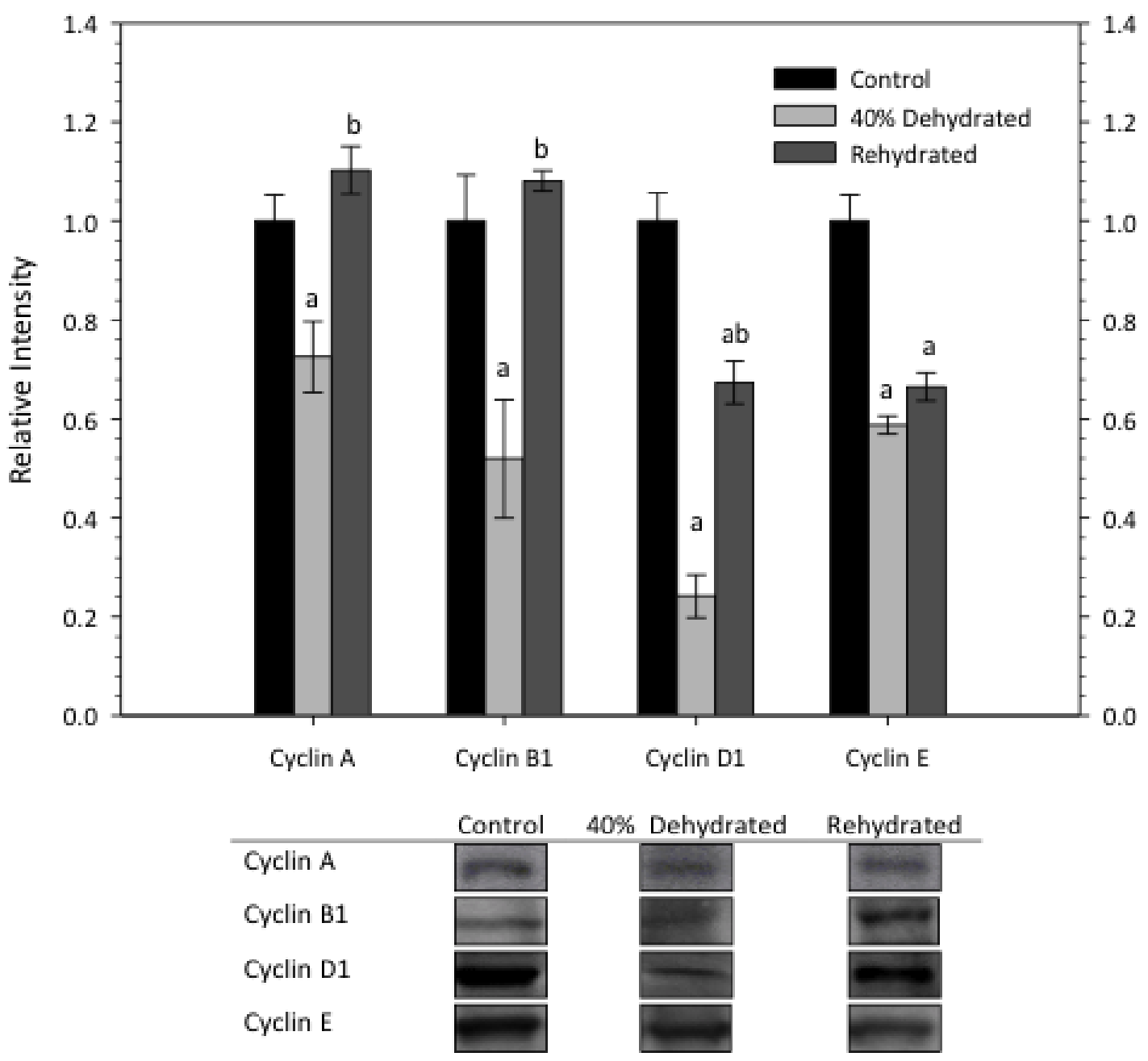

Figure 3.7. Effects of $40 \%$ dehydration and rehydration on protein levels of cyclins in wood frog liver. a - Significantly different from the corresponding control $(\mathrm{p}<0.05)$; $\mathrm{b}$ Significantly different from the $40 \%$ dehydrated value. Other information as in Figure 3.5 . 
Fig. 3.8A

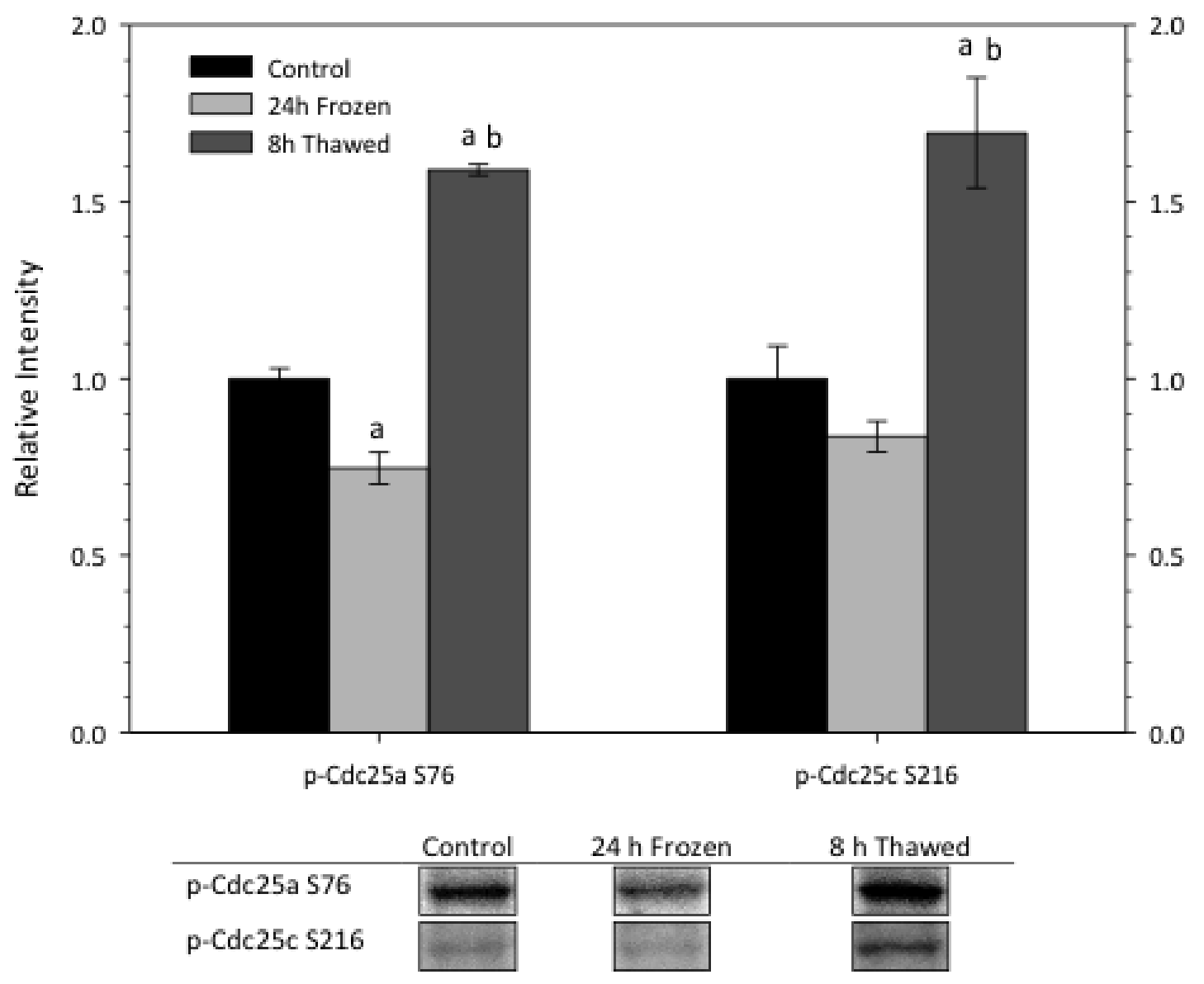


Fig. 3.8B

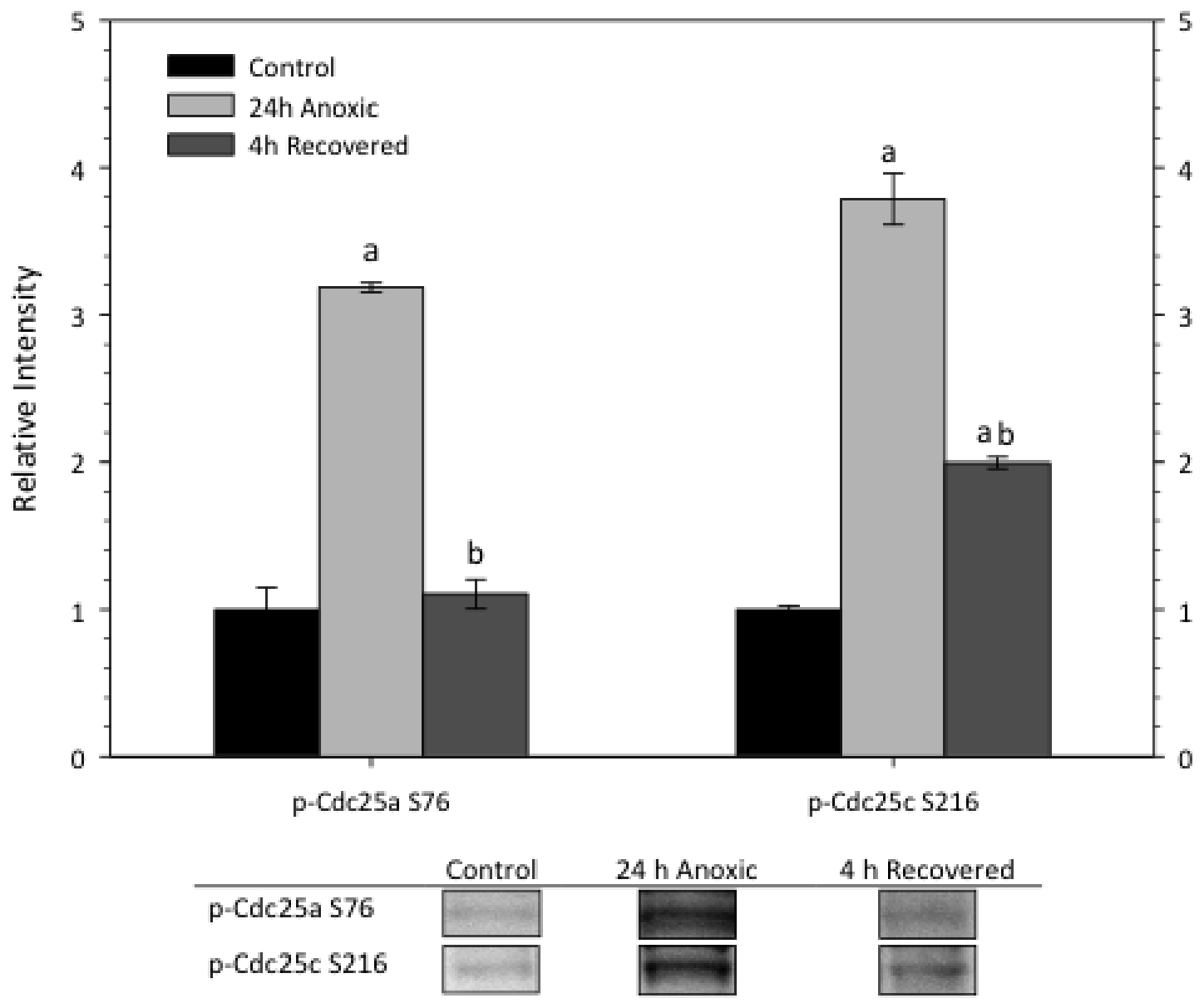


Fig. 3.8C

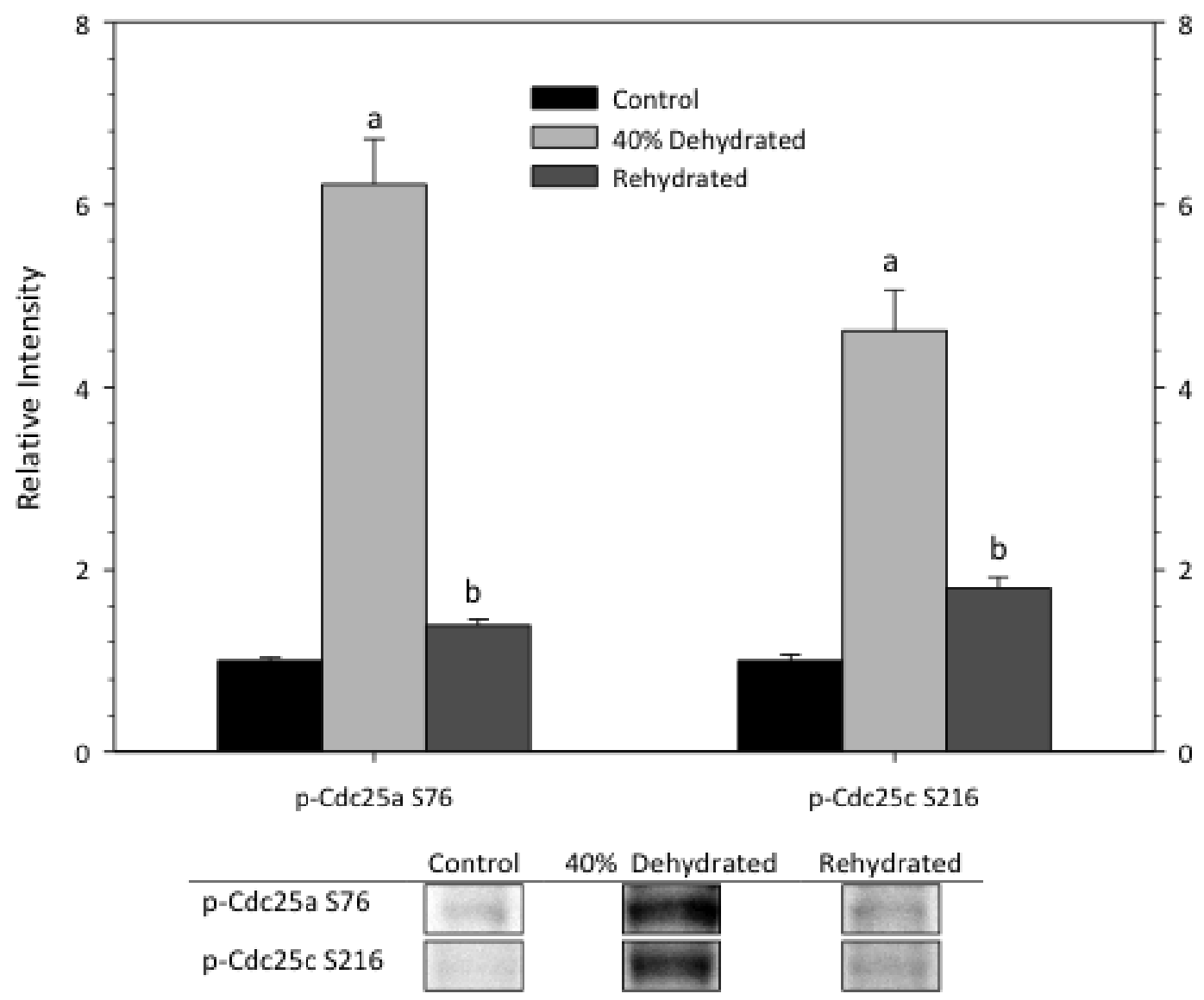

Figure 3.8. Effect of freezing, anoxia and dehydration stresses on the relative phosphorylation state of Cdc25a phosphorylated on Ser76 (p- Cdc25a S76) and Cdc25c phosphorylated on Ser216 (p-Cdc25c S216) in liver of wood frogs. (A) Responses to $24 \mathrm{~h}$ freezing and $8 \mathrm{~h}$ thawing. (B) Responses to $24 \mathrm{~h}$ anoxia and $4 \mathrm{~h}$ aerobic recovery. (C) Responses to $40 \%$ dehydration and rehydration. Histograms show standardized relative levels of phosphorylated protein under control, stressed and recovered conditions; data are means \pm SEM, $\mathrm{n}=3-4$ independent trials. Lower panels show representative immunoblot bands. a - Significantly different from the corresponding control $(p<0.05)$; b - Significantly different from the corresponding stressed value. 
Fig. 3.9A

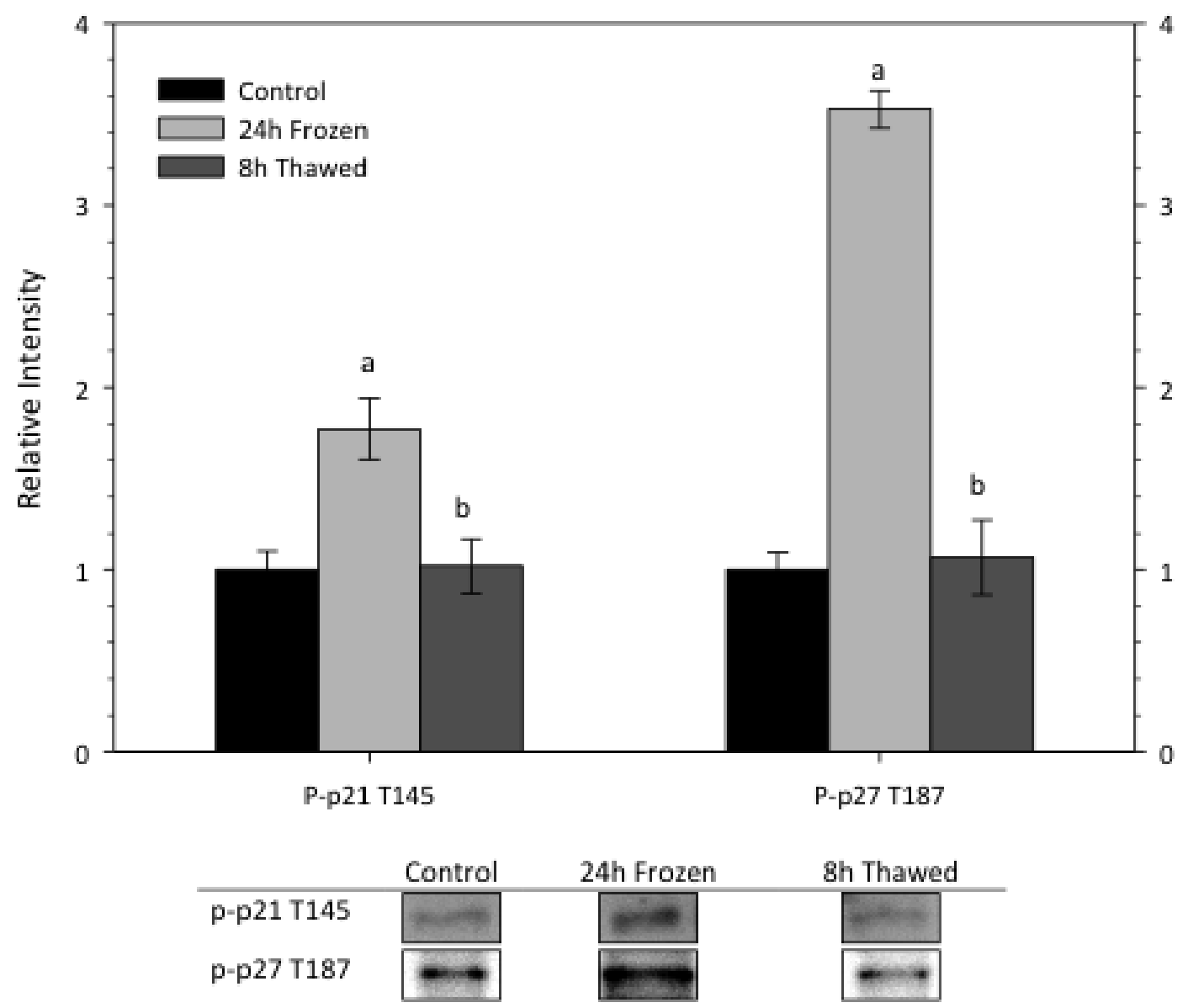


Fig. 3.9B

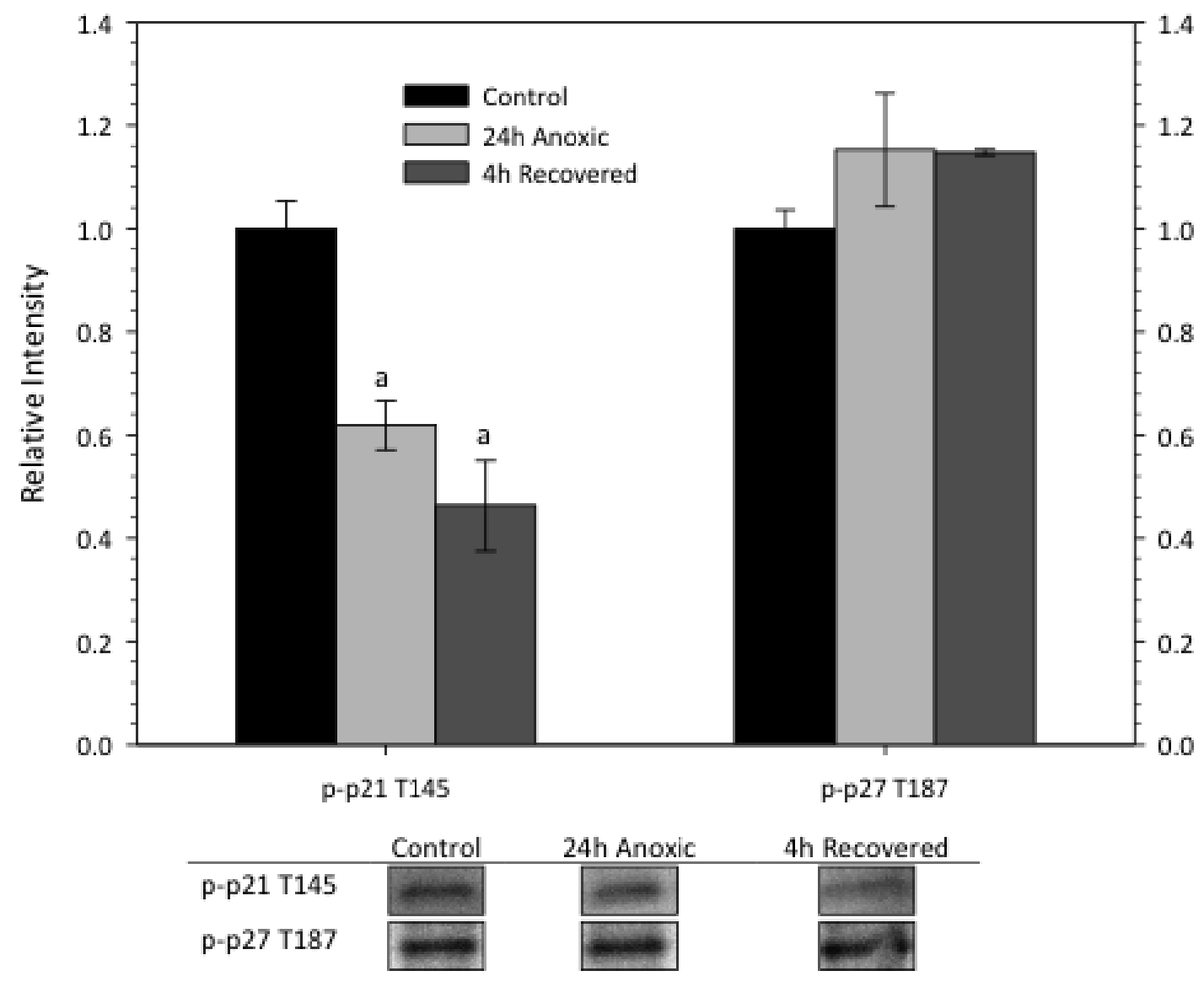


Fig. 3.9C

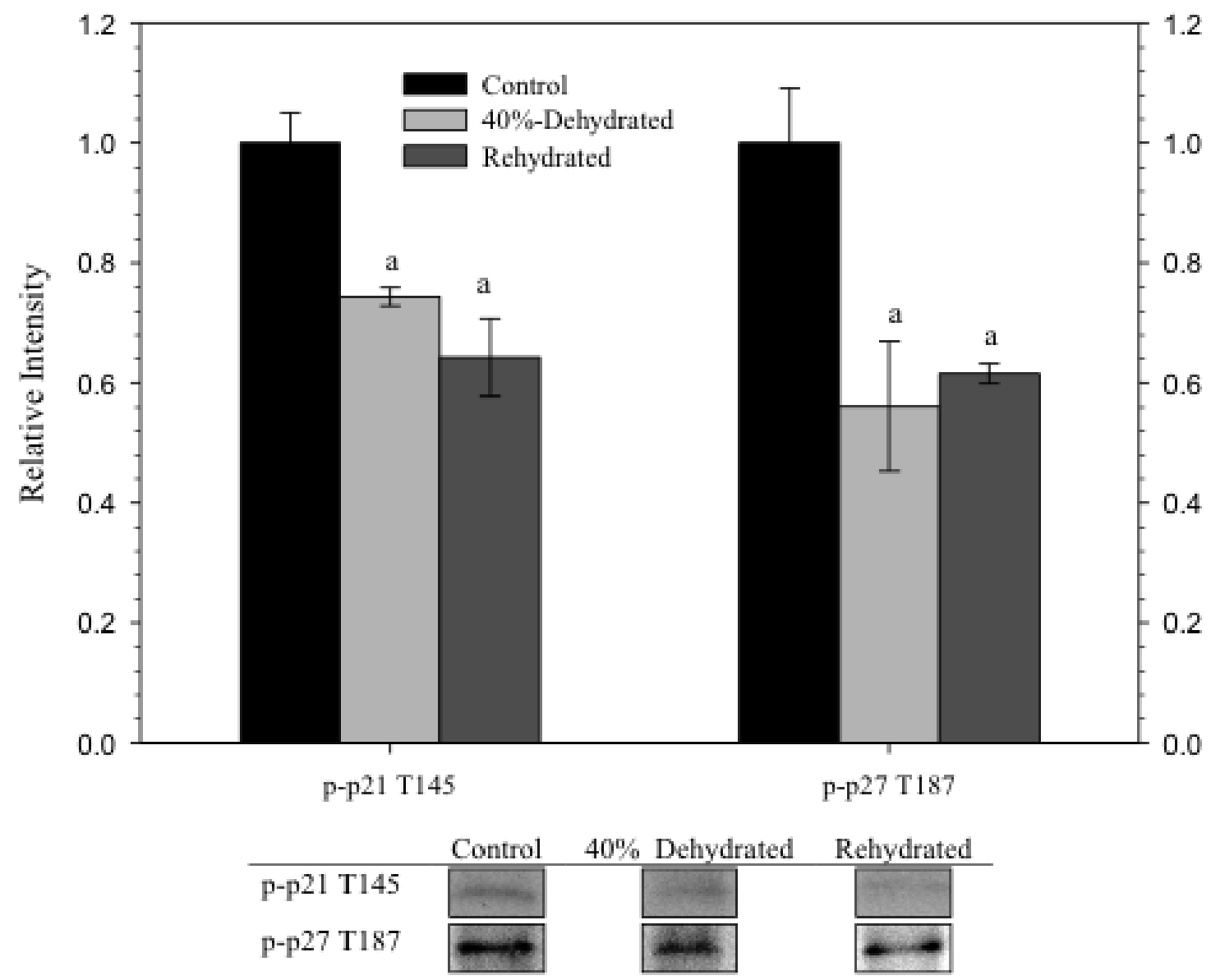

Figure 3.9. Effect of freezing, anoxia and dehydration stresses on the relative phosphorylation state of p21 phosphorylated on Thr145 (p-p21 T145) and p27 phosphorylated on Thr 187 (p-p27 T187) in wood frog liver. (A) Responses to $24 \mathrm{~h}$ freezing and $8 \mathrm{~h}$ thawing. (B) Responses to $24 \mathrm{~h}$ anoxia and $4 \mathrm{~h}$ aerobic recovery. (C) Responses to $40 \%$ dehydration and rehydration. Other information as in Figure 3.8. 


\section{Chapter 4 Regulation and response of Akt signaling}




\section{Introduction}

Akt is a core member of major intracellular signaling cascades that are involved in the regulation of metabolic events including protein synthesis, glucose and energy metabolism, cell survival and cell proliferation (Coffer and Woodgett, 1991; Jones, et al., 1991; Bellacosa et al., 1991; Sen et al., 2003; Cheng et al., 2005; Zhang et al., 2011). These cascades regulate crucial cellular processes either directly or indirectly via Akt-conducted phosphorylation events on transcription factors, protein kinases and other proteins. Extensive studies of Akt over many years have established a general principle that active Akt signaling promotes cell survival and proliferation, and inhibits apoptosis (Zhang et al., 2011). Therefore, I hypothesized an involvement of Akt in freezing survival of wood frogs, involving both a freeze-responsive regulation of Akt and the actions of Akt on downstream targets during freeze/thaw. Known functions of Akt in other systems including control over glucose transporters and anti-apoptosis actions suggest that Akt may have a role to play in both cryoprotectant metabolism and cell survival during freezing.

Activation of Akt depends on phosphorylation of two main residues, Ser-473 and Thr-308 (Alessi et al., 1997). The Thr-308 residue is located within the kinase domain and phosphorylation at this site stimulates the protein kinase activity of Akt. By contrast, phosphorylation on Ser-473 serves a regulatory role. The process of Akt activation takes place mainly at the plasma membrane where several key regulators including PI3K, PTEN (phosphatase and tensin homologue), and $\mathrm{PI}(3,4) \mathrm{P}_{2}$ (phosphatidylinositol-3, 4bisphosphate) are located (Oudit and Penninger, 1999). The current model of Akt activation proposes that active $\mathrm{PI} 3 \mathrm{~K}$ phosphorylates $\mathrm{PI}(3,4) \mathrm{P}_{2}$ to produce PIP3 
(phosphatidylinositol-3,4,5-trisphosphate) which recruits both Akt and PDK-1

(phosphoinositide-dependent kinase 1) to the plasma membrane. In close proximity to its substrate, PDK-1 phosphorylates Akt at Thr-308 to enhance its kinase activity. Notably, PDK-1 activity is regulated by phosphorylation on its Ser-241 residue, which may be phosphorylated by other regulatory kinases or via an autophosphorylation event (Casamayor and Alessi, 1999). Akt phosphorylation on Ser-473 is accomplished by TORC2 (Hersko and Mueckler, 2005) and studies suggest that this enhances Akt kinase activity (Yang et al., 2002; Sarbassov et al., 2005; Hart and Vogt, 2011; Huang et al., 2011). Akt is also affected by the phosphatase PTEN that converts PIP3 to PI(4,5) $\mathrm{P}_{2}$ thereby depleting cellular PIP3 content (Maehama and Dixon, 1998) and acting as a negative regulator of Akt (Fig. 4.1).

With respect to the crucial regulatory roles of PI3K and PTEN on Akt signaling, the opposing actions of these enzymes on the phosphorylation status of PIP3 means that close coordination is needed. Recent studies have focused on the involvement of microRNA species as a mechanism of providing post-transcriptional control over this reciprocal enzyme pair. Significant changes in the relative expression levels of selected miRNA species have been documented in response to freezing in the wood frog (Biggar et al., 2009), and hence the potential exists for post-transcriptional regulation of PI3K and PTEN by miRNA during freezing as well. PI3K regulation by miRNA appears to be indirect. Fish et al. (2008) showed that the major PI3K inhibitor, PI3KR2 (Phosphoinositide-3-kinase, regulatory subunit $2 \beta$, also known as $\mathrm{p} 85 \beta$ ), is a direct target of miR-126 and proposed that miR-126 plays a role in the regulation of PI3K in response to VEGF (vascular endothelial growth factor) in mammalian cells. Transcripts of pten are 
also controlled on a post-transcriptional level via miRNAs, the key ones being miR-26a and miR-217. Elevated miR-26a expression levels were associated with PTEN degradation, contributing to platelet-derived growth factor (PDGF)-dependent control of Akt signaling in gliomas (Huse et al., 2009). Furthermore, transforming growth factor $\beta$ (TGF- $\beta$ ) activation leads to an up-regulation of miR-217 which directly inhibits pten expression, thereby activating Akt activity (Kato et al., 2009).

This chapter reports studies of the gene expression patterns and phosphorylation states of core components of Akt signaling in wood frog liver (with selected data on other tissues) in response to whole body freezing and thawing. The involvement of miRNA action in regulating gene expression patterns was also examined along with an analysis of FOXO1, a downstream target of Akt signaling that is important in the anti-apoptosis actions of Akt. Furthermore, to examine the stress specific nature of Akt signaling, the responses of Akt to two components of freezing (anoxia and dehydration) were also evaluated. The study demonstrates that stress- and organ-specific regulation of Akt signal transduction is an integral part of freezing survival by wood frogs.

\section{Results}

\section{Effects of freezing on Akt expression in wood frogs}

Protein levels of Akt as well as relative phosphorylation of Akt at Ser-473 and Thr-308 were assessed by immunoblotting in liver, hind leg skeletal muscle, kidney and heart of wood frogs comparing control $\left(5^{\circ} \mathrm{C}\right.$ acclimated), $24 \mathrm{~h}$ frozen (at $\left.-2.5^{\circ} \mathrm{C}\right)$ and $8 \mathrm{~h}$ thawed $\left(\right.$ at $5^{\circ} \mathrm{C}$ ) experimental conditions. The polyclonal mammalian antibodies used 
cross-reacted with a single band at $\sim 46 \mathrm{kDa}$ corresponding to the known molecular weight of Akt in other species. Fig. 4.2A shows that after $24 \mathrm{~h}$ freezing exposure total Akt protein in heart decreased significantly to $56 \pm 11.1 \%$ of the control value whereas Akt content in the other three tissues did not change. After an $8 \mathrm{~h}$ thaw, Akt protein remained low in heart and also decreased significantly in skeletal muscle; levels were 51 $\pm 2.3 \%$ and $53 \pm 12.5 \%$ of the corresponding control values, respectively.

The effects of freeze-thaw on the relative phosphorylation levels of Akt at Ser473 are shown in Fig. 4.2B. Freezing stimulated a strong significant decrease in Ser-473 phosphorylation in muscle, kidney and heart to $66 \pm 4.6 \%, 32 \pm 0.89 \%$ and $45 \pm 2.8 \%$ of the corresponding control values, respectively. After an $8 \mathrm{~h}$ thaw, levels returned to control values in muscle and heart and exceeded control values in kidney $(1.3 \pm 0.04$ fold higher than controls). Liver showed a very different response to freezing; phospho-Akt Ser-473 levels increased significantly by $1.5 \pm 0.11$ fold after $24 \mathrm{~h}$ freezing and rose further to $1.96 \pm 0.11$ fold higher than controls after an $8 \mathrm{~h}$ thaw. Relative levels of phospho-Akt Thr-308 also changed over freeze-thaw (Fig. 4.2C). Liver showed a $1.86 \pm$ 0.07 fold increase in phospho-Akt Thr-308 content in response to freezing and this remained high during thawing ( $1.33 \pm 0.08$ fold over control values). Kidney showed a strong decrease in phospho-Thr-308 content during freezing to just $16 \pm 3 \%$ of the control value whereas the level rose strongly after thawing to $1.46 \pm 0.02$ fold over control values. After $24 \mathrm{~h}$ freezing, relative phospho-Akt Thr-308 levels in heart dropped significantly to $66 \pm 2.9 \%$ of the control value and remained low $(61 \pm 2.8 \%)$ after an $8 \mathrm{~h}$ thaw. Phospho-Akt Thr-308 content did not change in skeletal muscle over freeze-thaw experimental conditions. 
Effects of anoxia and dehydration on Akt expression in liver

Two of the main components of a freezing event are cellular dehydration (due to water exit into extracellular spaces) and ischemia/anoxia (caused by the cessation of heart beat and the freezing of plasma). Hence, the molecular responses to anoxia or dehydration stresses are of value in exploring the triggers and the functions of Akt in response to freeze-thaw. Since liver exhibited a unique Akt activation under freezing exposure out of four tissues tested, the effects of anoxia and dehydration on Akt were also examined in hopes of identifying the stress component responsible for the Akt response. Fig. 4.3A shows the responses of Akt in wood frog liver to $24 \mathrm{~h}$ anoxia exposure and $4 \mathrm{~h}$ aerobic recovery. Total Akt protein, phospho-Akt Ser-473 and phosphoAkt Thr-308 contents all decreased significantly in response to $24 \mathrm{~h}$ anoxia; levels were $62 \pm 7.8 \%, 18 \pm 0.71 \%$ and $24 \pm 4 \%$, respectively, of corresponding control values. However, after $4 \mathrm{~h}$ aerobic recovery all parameters rebounded strongly; phospho-Akt Ser473 content returned to control values whereas total Akt and phospho-Akt Thr-308 levels rose to $1.7 \pm 0.094$ and $1.8 \pm 0.11$ fold higher than controls, respectively.

Fig. 4.3B shows a similar Akt response to dehydration and rehydration in liver. Dehydration to $40 \%$ of total body water lost led to strongly suppressed levels of total Akt, phospho-Akt Ser-473 and phospho-Akt Thr-308; values dropped significantly to just $26 \pm$ $2.1 \%, 12 \pm 0.74 \%$ and $41 \pm 6 \%$ of the corresponding control values. After rehydration, total Akt protein content returned to control values, p-Akt Ser-473 content increased to $58 \pm 2.7 \%$ of the control level, and phospho-Akt Thr-308 levels rose to $1.4 \pm 0.01$ fold higher than controls. 
Effects of freezing on the nuclear content of Akt in liver

In order to trace the downstream effects of Akt activation in the liver of frozen frogs, the effects of freeze-thaw on the nuclear content of Akt and its phosphorylated forms (Ser-473 and Thr-308) were assessed using liver nuclear extracts (Fig. 4.4) (See Chapter 2 for the details of nuclear extraction procedure). Freezing had no effect on the total amount of Akt protein detected in nuclear extracts of liver. However, the relative amount of phospho-Akt Ser-473 and Thr-308 increased in nuclear fractions of $24 \mathrm{~h}$ frozen frogs by $1.3 \pm 0.06$ and $1.3 \pm 0.04$ fold, respectively. After $8 \mathrm{~h}$ thawing, total Akt protein present in the nuclear fraction of liver increased strongly by $4.6 \pm 0.29$-fold. Relative levels of phospho-Akt Ser-473 and Thr-308 also increased in the nuclear fraction after thawing with fold changes of $1.7 \pm 0.06$ and $2.47 \pm 0.08$, respectively, as compared with corresponding control values (Fig. 4.4).

\section{Effects of freezing on PDK-1 expression in liver}

To investigate the potential upstream regulation of Akt activation, total PDK-1 protein levels as well as its relative phosphorylation state at Ser-241 were analyzed via immunoblotting in liver of control, $24 \mathrm{~h}$ frozen and $8 \mathrm{~h}$ thawed wood frogs (Fig. 4.5). Polyclonal antibodies for total PDK-1 and phospho-PDK-1 Ser-241 crossreacted with a single protein band at a molecular weight corresponding to the known value for PDK-1 in other vertebrates. Relative PDK-1 protein levels in liver did not change after $24 \mathrm{~h}$ freezing exposure but decreased to $63 \pm 8.6 \%$ of the control value after 8 h thawing (Fig. 
4.5). On the other hand, phospho-PDK-1 Ser-241 content rose by $1.9 \pm 0.18$ fold after 24 $\mathrm{h}$ freezing but returned to control values after $8 \mathrm{~h}$ thawing (Fig. 4.5).

Effects of freezing on PTEN gene/protein expression in liver

Figure 4.6 shows the effect of wood frog freezing and thawing on the protein and mRNA levels of the PTEN phosphatase that regulates Akt. Total PTEN protein levels fell to $55 \pm 9.2 \%$ of the control value in liver in response to $24 \mathrm{~h}$ freezing whereas $8 \mathrm{~h}$ thawing led to a partial recovery of PTEN towards control levels. Relative transcript levels of the pten gene were strongly elevated by $2.6 \pm 0.32$ fold in frog liver after $24 \mathrm{~h}$ freezing exposure. Levels fell during thawing but remained $1.6 \pm 0.07$ fold higher than control values. Representative bands of pten PCR amplified products in liver from control, $24 \mathrm{~h}$ frozen and $8 \mathrm{~h}$ thawed frogs are also shown in Fig. 4.6A with corresponding bands for $\beta$-actin amplified from the same RNA samples (GeneBank accession number for $\beta$-actin: KC438290).

A 425 bp partial sequence of pten was obtained (GenBank accession number for pten: KC438291) encoding a peptide sequence consisting of 141 amino acid residues. Using the western clawed frog sequence as a reference point (Genbank accession number: NP_001116943.1), the wood frog peptide sequence was found to correspond to an $\mathrm{N}$-terminal portion of the protein aligning with residues $21-161$. Fig. 4.6B and $\mathrm{C}$ show a homology tree and alignment of this partial PTEN amino acid sequence in wood frog compared with the sequences for the western clawed frog (Xenopus tropicalis; Genbank accession number NM_001090362), chicken (Gallus gallus; Genbank accession number XP_421555.2), human (Homo sapiens; Genbank accession number ADM26757.1, 
PTEN-like protein) and zebrafish (Danio rerio; Genbank accession number:

NP_001001822.1). The segment of PTEN retrieved from the wood frog showed 98\% similarity with the western clawed frog sequence and $94 \%$ with the chicken sequence. It also showed high sequence conservation with human and zebrafish, with percentage similarities of $93 \%$ and $88 \%$, respectively.

Effects of freezing on miRNA expression in liver

Upstream regulators of the Akt pathway are under control by microRNAs. The expression of pten transcripts is targeted by miR-26a and miR-217 whereas PI3K stability is indirectly regulated by miR-126. To examine the possible involvement of posttranscriptional regulation on liver Akt by miRNA, transcript levels of these three miRNAs were examined in wood frog liver under freezing and thawing conditions. A stemloop primer system and RT-PCR were used as described previously (Biggar et al., 2011). Relative transcript levels of miR-26a, miR-126 and miR-217 all increased significantly during freezing by $1.2 \pm 0.03,1.36 \pm 0.07$ and $1.3 \pm 0.03$-fold, respectively (Fig. 7). Levels of all three remained elevated after $8 \mathrm{~h}$ thawing although miR-126 showed a significant decrease from peak values; transcript levels were $1.2 \pm 0.06$ (miR26a), $1.3 \pm 0.05$ (miR-126) and $1.49 \pm 0.06$ (miR-217) fold higher than corresponding controls. 
FOXO1 is an Akt downstream target and is phosphorylated by Akt at Ser-256. The effects of freeze-thaw on the relative levels of total FOXO1 and phospho- FOXO1 Ser-256 in wood frog liver are shown in Fig. 4.8. Freezing had no effect on total FOXO1 levels in liver but in $8 \mathrm{~h}$ thawed frogs FOXO1 content was reduced to $69.4 \pm 1.1 \%$ of the control value. Phospho-FOXO1 Ser-256 content in liver of frozen frogs was $1.4 \pm 0.05$ fold higher than in controls and rose further to $1.6 \pm 0.12$ fold higher than controls after 8 $\mathrm{h}$ thawed.

\section{Discussion}

In order for wood frogs to survive whole body freezing, both energy conservation and cryoprotective strategies need to be coordinated. First of all, energy expenditures are suppressed and reprioritized by slowing or shutting down biological process that demand a large input of ATP; this extends survival time in the ischemic frozen state (Storey, 1987a). Biochemical adaptations are also put in place to specifically tackle problems related to freezing; these include the production of extremely high levels of glucose as a colligative cryoprotectant and the differential regulation of multiple genes/proteins, including the production of several novel proteins (Storey, 2004a). Multiple studies have examined the specific biochemical responses of liver given its vital role in surviving freeze-thaw cycles (Storey and Storey, 1984, 1996, 2004a; Storey, 1987a; Storey et al., 1992; Storey and Mommsen, 1994; Conlon et al., 1998; Holden and Storey, 2000; Dieni et al., 2012; Chapter 3). For example, liver has the only large store of glycogen that can support the production and export of glucose used for cryoprotection by all other organs (Storey and Storey, 1984; Storey, 1987a). Initiation of glucose synthesis and 
output from liver begins within as little as 2 minutes after ice nucleation at peripheral sites on the skin (Storey and Storey, 1985), long before any significant amount of ice forms. Therefore, the link between peripheral ice nucleation and triggering of liver cryoprotective responses must involve intraorgan and intracellular signal transduction pathways (Storey and Storey, 1996) as well as crucial modifications to cell signaling that allow wood frogs to override the normal homeostatic controls on glycogen/glucose metabolism in vertebrate liver.

The present study analyzed the Akt pathway by investigating gene and protein expression and phosphorylation states of core components in the pathway. In general, wood frog muscle, kidney and heart showed signs of Akt pathway inhibition in response to freezing followed by a restoration of Akt activity upon thawing. In this capacity, total Akt protein and the relative amounts of the two phospho-Akt forms were either unchanged or reduced significantly in response to freezing in these organs and recovered after $8 \mathrm{~h}$ thawed (Fig. 4.2A). Given that phosphorylation at Ser-473 and Thr-308 are crucial to Akt activation, the observed reduction in Akt phosphorylation in these three organs is generally consistent with reduced Akt function in the frozen state. However, some tissue specific Akt suppression patterns were noted. For example, skeletal muscle only showed a decrease in phospho-Akt Ser- 473 content (66\% of control value) after $24 \mathrm{~h}$ freezing exposure, but not in total protein or Thr-308 phosphorylation (Fig.4.1) and phospho-Akt Ser-473 returned to control level in $8 \mathrm{~h}$ thawed animals (Fig. 4.1B). These data suggest that freeze-thaw responses by Akt in muscle may be regulated by TORC2, which is the upstream kinase that acts at Ser-473 (Hersko and Mueckler, 2005). The kidney data indicated that regulation of Akt was solely dependent on the phosphorylation 
state of the enzyme since both Ser-473 and Thr-308 phosphorylation levels dropped significantly whereas total protein was not affected by $24 \mathrm{~h}$ freezing (Fig. 4.1). In the case of heart, however, total Akt protein content dropped to about half of the control value after $24 \mathrm{~h}$ freezing exposure as did the levels of both phospho-forms (reduced to $45-66 \%$ of control values). After $8 \mathrm{~h}$ thawing, only Ser-473 phosphorylation levels recovered to control values, with both total and Thr308 phosphorylated Akt remaining suppressed (Fig. 4.1), suggesting that recovery of Akt signaling in heart takes longer than in muscle and kidney. Overall, the results for these three tissues indicate reversible suppression of Akt function during freezing. Since most of the downstream events that are positively controlled by active Akt are ATP-expensive biosynthetic processes (e.g. protein synthesis, cell proliferation, etc.), this suggests that a reduction in Akt signaling may be a crucial factor in coordinating a freeze-induced global suppression of metabolism in wood frog organs. Reduced Akt signaling would also lead to reduced phosphorylation of glycogen synthase kinase 3 (GSK-3) and, thereby, allow dephosphorylated GSK-3 to inhibit glycogen synthase. This could have a crucial action in non-hepatic tissues - a block on the conversion of cryoprotectant glucose into glycogen over the duration of the freeze.

In liver, Akt signaling showed a unique response to freezing that were generally opposite to those of the other tissues. Total Akt protein levels in liver did not change over freeze-thaw but amounts of both phospho-enzyme forms increased significantly during freezing and remained high after thawing (Fig. 4.1). Several studies have shown that active Akt signaling promotes cell survival under stresses such as DNA damage, oxidative stress, etc. (Horic et al., 2008; Xu et al., 2010). Protection of liver function 
would be crucial for wood frogs because liver has the major role in producing cryoprotective defenses for the whole animal, not only glucose but also novel protective proteins (Storey, 2004a). During freezing, liver and plasma glucose levels can rise to 150-300 $\mathrm{mM}$ as compared with control values of only about $\sim 5 \mathrm{mM}$ (Storey and Storey, 2004a; Storey, 1987a, 2004a). Thus, adaptation strategies need to be taken to maintain liver function. For example, whereas wood frog liver showed suppression of Akt activity in response to anoxia or dehydration stresses (Fig. 4.2), this did not occur during freezing which might allow liver to retain an active capacity for biosynthesis, cell cycle activity, or even suppression of apoptosis while frozen (Chapter 3). The same cell cycle-focused study also proposed that Akt activation would occur in liver during freezing due to an observed increase in the level of phosphorylated p21 (Thr-145), a well-known regulator of cell cycle and Akt target. The present results corroborate this prediction.

Enhanced Akt signaling may not only help to preserve cell cycle progression, but also promote cell survival. In this capacity, Chapter 3 reported an increase of Aktdependent phosphorylation on p21 in liver of frozen wood frogs, suggesting potential involvement of Akt as an apoptosis inhibitor due to the pro-survival function of $\mathrm{p} 21$ when phosphorylated . Besides p21, Akt signaling can suppress programmed cell death in other ways including direct inhibition of pro-apoptotic proteins, BAD and caspase 9 , as well as by inhibiting FOXO1 signaling, thereby suppressing transcription of cell death-related genes such as the death signal cytokine Fas L (Fas ligand) (Brunet et al., 1999). When activated, Akt is free to move away from the plasma membrane to function at multiple subcellular locations, including moving into the nucleus (Adjekovic et al., 1997). In the nucleus, Akt phosphorylates FOXO1 on several sites and this promotes FOXO1 binding 
to 14-3-3 protein, its subsequent expulsion from the nucleus, and FOXO1 degradation in an ubiquitin-dependent manner (Zhao et al., 2004; Huang et al., 2005). This appears to be one mode of Akt action in wood frog liver since the amount of phosphorylated Akt was elevated in the nuclear fraction of liver during both freezing and thawing (Fig. 4.3). Phosphorylation of FOXO1 on Ser-256 by Akt is particularly important for triggering its ubiquitin-dependent proteolysis and analysis of this phospho-site showed a progressive increase in Ser-256 phosphorylation during freezing and thawing (Fig. 4.7). Furthermore, a strong decrease in total FOXO1 content in liver of $8 \mathrm{~h}$ thawed frogs correlated with a >4-fold increase in total nuclear Akt (and elevated phospho-Akt) (Fig. 4.3) implicating Akt in the suppression of FOXO1 signaling. Therefore, it appears that Akt may be involved in promoting cell survival in wood frog liver through both p21- and FOXO1dependent mechanisms. This suggests a strong focus on anti-apoptosis, pro-survival actions by Akt in wood frog liver during the thawed recovery period after freezing. Reestablishment of liver homeostasis is critical because this organ is responsible for clearance of the cryoprotectant after thawing and restoring glucose into liver glycogen granules as well as clearing various metabolic end products (e.g. lactate) that accumulated during the freeze.

In order to explore the mechanisms behind liver Akt activation in frozen frogs, the current chapter investigated regulation of the enzyme by PDK-1 and PTEN. PDK-1 is responsible for phosphorylating Thr-308 on Akt, so the enhanced level of Thr-308 phosphorylation during freeze/thaw (Fig. 4.1C) implicates PDK-1 action. Indeed, phosphorylation of PDK-1 on Ser-241 increased nearly 2-fold in liver of frozen frogs (Fig. 4.4) showing activation of the kinase. Notably, total liver PDK-1 did not increase 
during freezing, as was also seen for Akt, which indicates the clear role of posttranslational modification in the regulation of freeze-responsive signal transduction and fits with an energy conservation strategy in the frozen (hypometabolic) state. After thawing, total PDK-1 in liver dropped to $\sim 60 \%$ of control but phospho-PDK-1 Ser-241 content remained near control values (Fig. 4.4), thereby indicating a relative increase in active phosphorylated PDK-1 content in liver of thawed frogs. This could support the corresponding high level of phospho-Akt Thr-308 in thawed frogs (Fig. 4.1C).

PTEN is effectively an inhibitor of Akt because it depletes PIP3 that recruits Akt and PDK-1 to the plasma membrane for activation. Hence, modulation of PTEN would also affect Akt action during freeze-thaw. After $24 \mathrm{~h}$ freezing, the relative protein level of PTEN in wood frog liver decreased to about half of the control value (Fig. 4.5) which would be consistent with activation of both Akt and PDK-1 in liver of frozen frogs. However, pten transcript levels actually rose in liver of $24 \mathrm{~h}$ frozen frogs and remained higher than control values after $8 \mathrm{~h}$ thawed. This suggested the possibility of posttranscriptional regulation of pten transcripts. In recent years, the key role of microRNAs in post-transcriptional control of gene expression has been recognized (Bartel, 2009) and differential expression of selected microRNA species has been documented during wood frog freezing (Biggar et al., 2009). Evidence has shown that miR-26a and miR-217 are closely related to inhibition of PTEN protein expression (Huse et al., 2009; Kato et al., 2009). The present data demonstrate that both of these miRNAs accumulated in wood frog liver after $24 \mathrm{~h}$ freezing exposure and that levels remained elevated after $8 \mathrm{~h}$ thawing (Fig. 4.6). Enhanced miRNA levels are consistent with translational inhibition of the specific mRNA targets. Hence, these data can explain the observed inconsistency 
between PTEN protein and pten transcript levels. Furthermore, the data showed increased levels of miR-126 during freezing (Fig. 4.6). This miRNA facilitates PI3K activation, suggesting that PI3K may be activated by miR-126 action during freezing at the same time as PTEN production is inhibited by miR-26a and miR-217. Upon $8 \mathrm{~h}$ thawing, liver levels of both miR-26a and miR-217 remained higher than control levels (Fig. 4.6), which may explain why only a moderate recovery was observed in PTEN protein levels in wood frog liver at this time (Fig. 4.5).

Insulin is one of the major Akt pathway activators. Given that glucose levels need to be maintained at high levels to provide cryoprotection to wood frogs throughout the freeze, it is possible that insulin exhibits distinct properties in the wood frog. Previous studies showed that wood frog insulin has unique structural features that could alter its interaction with its receptors during freezing (Conlon $\boldsymbol{e t}$ al., 1998). This was proposed as a possible mechanism to override normal glucose homeostasis, thereby promoting extreme hyperglycemia and cryoprotectant accumulation. In the context of the present study, the specific features of wood frog insulin might also contribute to the freezespecific behaviour of liver Akt, as compared with Akt responses to anoxia or dehydration. However, liver Akt activation in the frozen wood frog might also be insulinindependent. The miRNA data already indicated the involvement of alternative controls on liver Akt activation during freezing. Kato et al. (2009) proposed that miR-217 is responsive to TGF- $\beta$ activation which suggests that, via the effects of elevated miR-217 on PTEN expression, that TGF- $\beta$ could facilitate Akt activation during freezing. Furthermore, miR-26a is involved in platelet-derived growth factor (PDGF)-dependent 
activation of Akt (Huse et al., 2009), which may also implicate this pathway in freezeresponsive Akt activation.

Finally, a comment about Akt and the regulation of glycogen metabolism in wood frog liver during freezing is appropriate. One of the best-known actions of Akt is phosphorylation of GSK-3 on Ser-9 to inhibit this kinase and thereby increase glycogen synthase (GS) activity and mediate insulin-stimulated glycogen synthesis in liver (Embi et al., 1980; Grimes and Jope, 2001). However, despite freeze-responsive Akt activation in wood frog liver, GSK-3 is dephosphorylated and active in liver of frozen frogs (Dieni et al., 2012) and liver GS activity is strongly suppressed within minutes of the initiation of freezing (Russell and Storey, 1995). This fits with the opposite strong activation of glycogen phosphorylase that mediates the massive glycogenolysis that is needed to support glucose synthesis as the cryoprotectant. Hence, Akt (and likely also insulin signaling) is clearly "unhooked" from its role in regulating glycogen metabolism during freezing and an "override" control on GSK-3 must be applied that keeps this enzyme active and GS suppressed despite the extremely high glucose levels in liver of frozen frogs. A similar "override" control must also apply to glycogen phosphorylase (GP) to keep its activity high during freezing because GP and GS are normally reciprocally regulated to maintain glucose levels in cells within narrow limits (normally about 2-5 $\mathrm{mM}$ ) (Syed and Khandelwal, 2000). A potential candidate for this override function is the AMP-activated protein kinase (AMPK) that is activated by freezing in wood frog liver (Rider et al., 2000). However, a definitive answer to the question of how this highly unusual freeze-responsive hyperglycemia is regulated is still unknown. The responses of wood frog liver Akt to anoxia or dehydration stresses (Fig. 4.2) show that the inhibition 
of Akt (reduction in both total Akt protein and phosphoprotein levels) occurs in liver and is reversed again during recovery, similar to the results observed in the other three tissues in response to freezing stress. Both anoxia/ischemia and dehydration are components of the stresses experienced by cells/tissues during freezing and various adaptive responses to freezing resemble well-known responses to oxygen limitation or cell volume reduction (Storey, 2004a). However, the data for anoxia and dehydration effects on Akt expression in wood frog liver, that are not consistent to those seen during freezing, provide additional evidence for the presence of a freeze-specific "override" regulation of liver metabolism to specifically support freeze tolerance. This observation echoes previous studies that proposed that the phosphorylation status of Akt is temperature sensitive (van Dam et al., 2005). While Akt phosphorylation was inhibited in human and murine cell under hypothermic conditions (around $\left.20^{\circ} \mathrm{C}\right)($ van Dam et al., 2005; Oehler-Jänne et al., 2008), the freezing condition $\left(-2.5^{\circ} \mathrm{C}\right)$ seems to have tissue-specific effects on the pathway in the freeze tolerant wood frog.

In conclusion, the present study demonstrates tissue and stress specific responses by Akt in freeze tolerant wood frogs. Regulation of Akt (as well as up- and downstream actions) was primarily due to post-translational modification via reversible phosphorylation which supports a need for both (a) reversible control over freeze-thaw cycles, and (b) control that involves minimal energy expenditure in the energy-limited ischemic frozen state. Since many of the downstream effects of Akt signaling regulate high energy cellular events such as cell survival, proliferation and growth, it is not surprising that the pathway was suppressed in muscle, kidney and heart in the ischemic frozen state, potentially as part of an overall metabolic rate depression response to stress. 
Akt signaling was also suppressed in liver under anoxia and dehydration stresses (which are two components of freezing) but Akt was specifically activated in liver during freezing. Akt activation was accompanied by changes in PDK-1, PTEN and microRNAs in liver of frozen frogs that also clearly support a freeze-responsive activation of Akt. Hence, all data indicate a novel role for Akt signaling in liver metabolism during freezing and this role may be in coordinating anti-apoptosis actions in liver during freezing via both p21 (Chapter 3) and FOXO1-dependent mechanisms. 
Fig. 4.1A

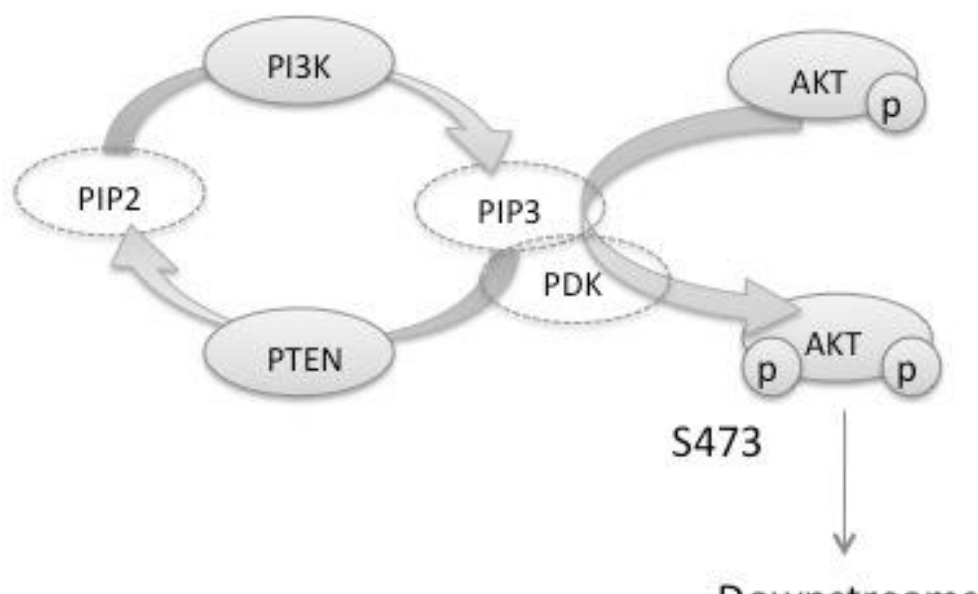

Downstreams

Fig. 4.1B

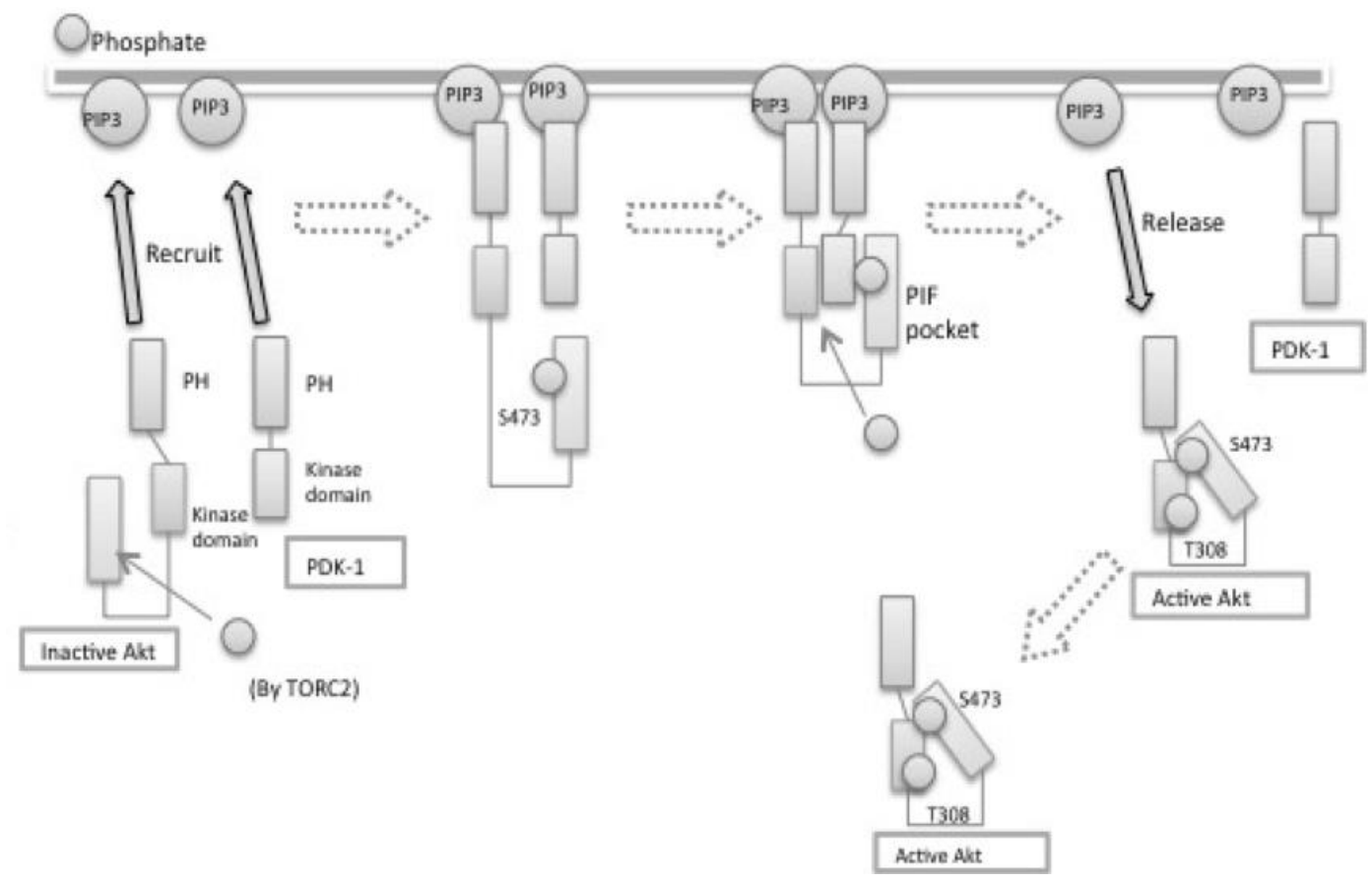

Fig. 4.1. Activation of Akt. (A) PI3K phosphorylates PIP2 to produce PIP3, which is required for Akt activation; (B) mechanism of Akt activation. Akt is first phosphorylated at Ser-473 and then recruited by PIP3 with PDK-1 to plasma membrane. PDK-1 phosphorylates Akt at Thr-308 to stimulate the kinase activity of the protein. After phosphorylation, active Akt is released from the membrane to regulate the subsequent downstream targets. PH: Pleckstrin homology domain 
Fig. $4.2 \mathrm{~A}$
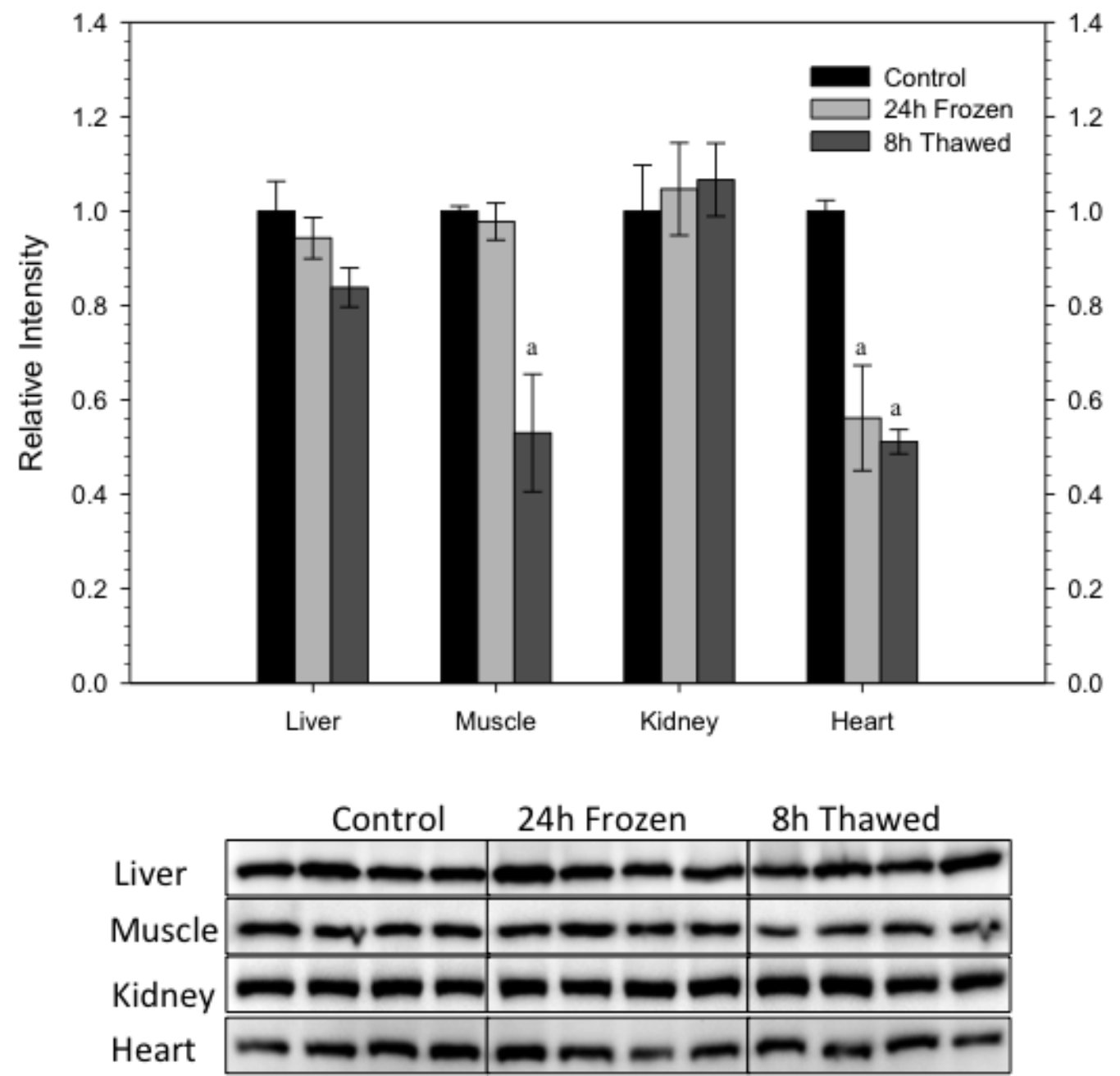
Fig. 4.2B
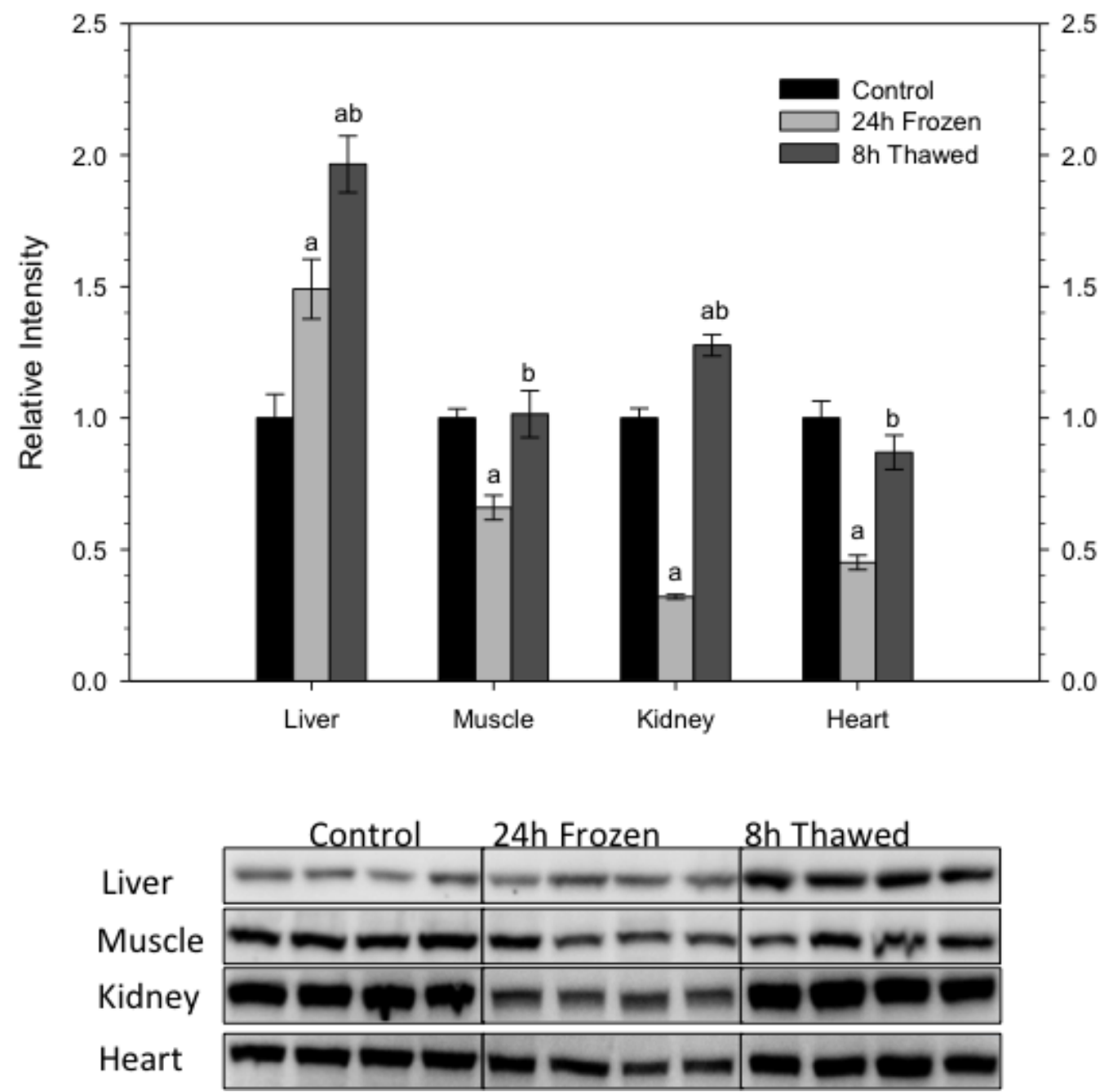
Fig. 4.2C
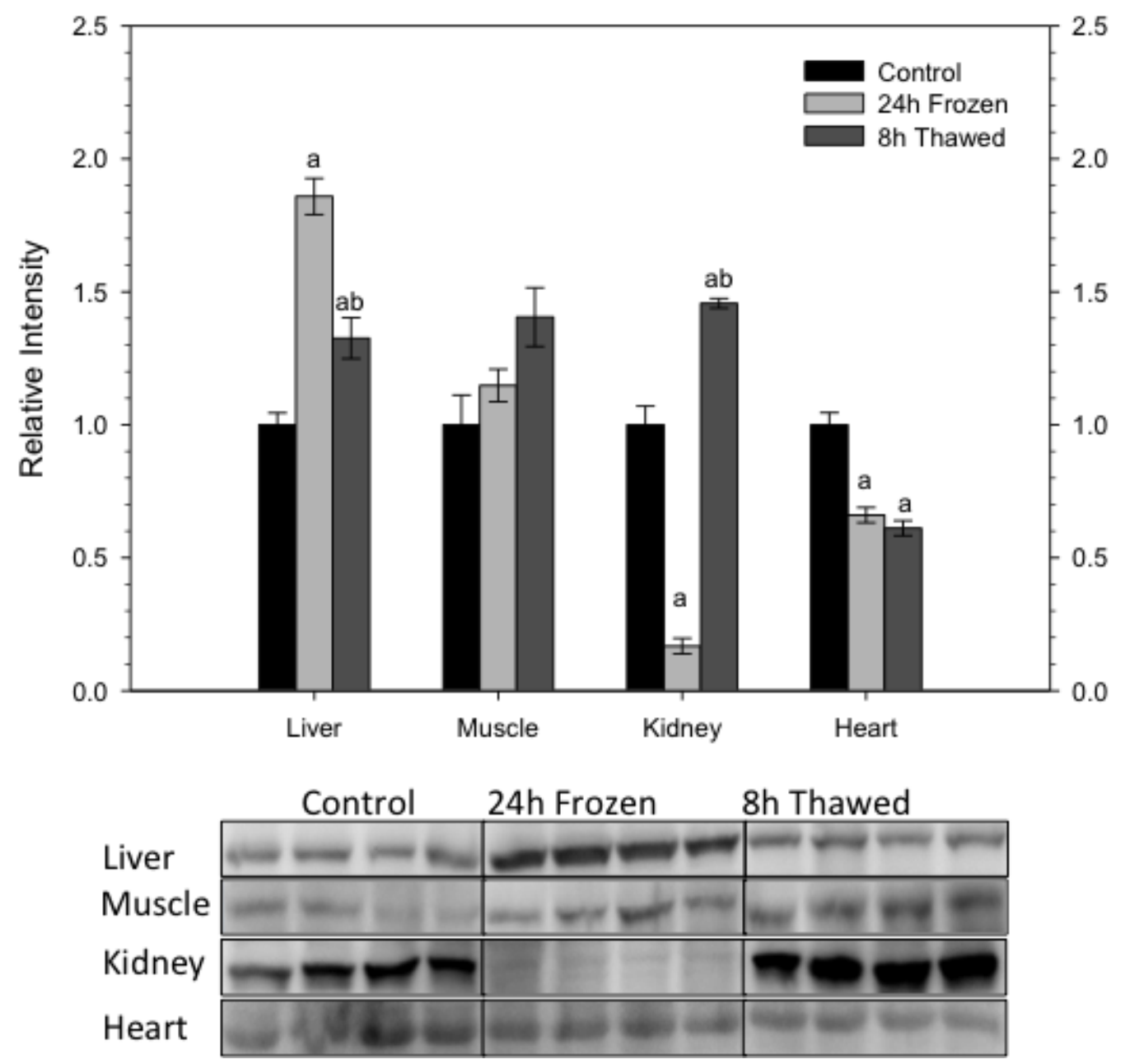

Fig. 4.2. Effects of $24 \mathrm{~h}$ freezing and $8 \mathrm{~h}$ thawing on protein levels of Akt as well as relative phosphorylation of Akt at Ser-473 and Thr-308 in wood frog liver, skeletal muscle, kidney and heart. (A) Total Akt protein levels. (B) Phospho-Akt Ser-473 level. (C) Phospho-Akt Thr-308 levels. Upper portion of each figure is the histogram showing standardized relative protein levels under control, $24 \mathrm{~h}$ frozen and $8 \mathrm{~h}$ thawed conditions; data are means \pm S.E.M., $n=3-4$ independent trials on tissue from different animals. a Significantly different from the corresponding control $(\mathrm{P}<0.05)$; $\mathrm{b}$ - significantly different from the $24 \mathrm{~h}$ frozen value. Lower portion of the figure shows representative immunoblot bands from each group. 
Fig. $4.3 \mathrm{~A}$
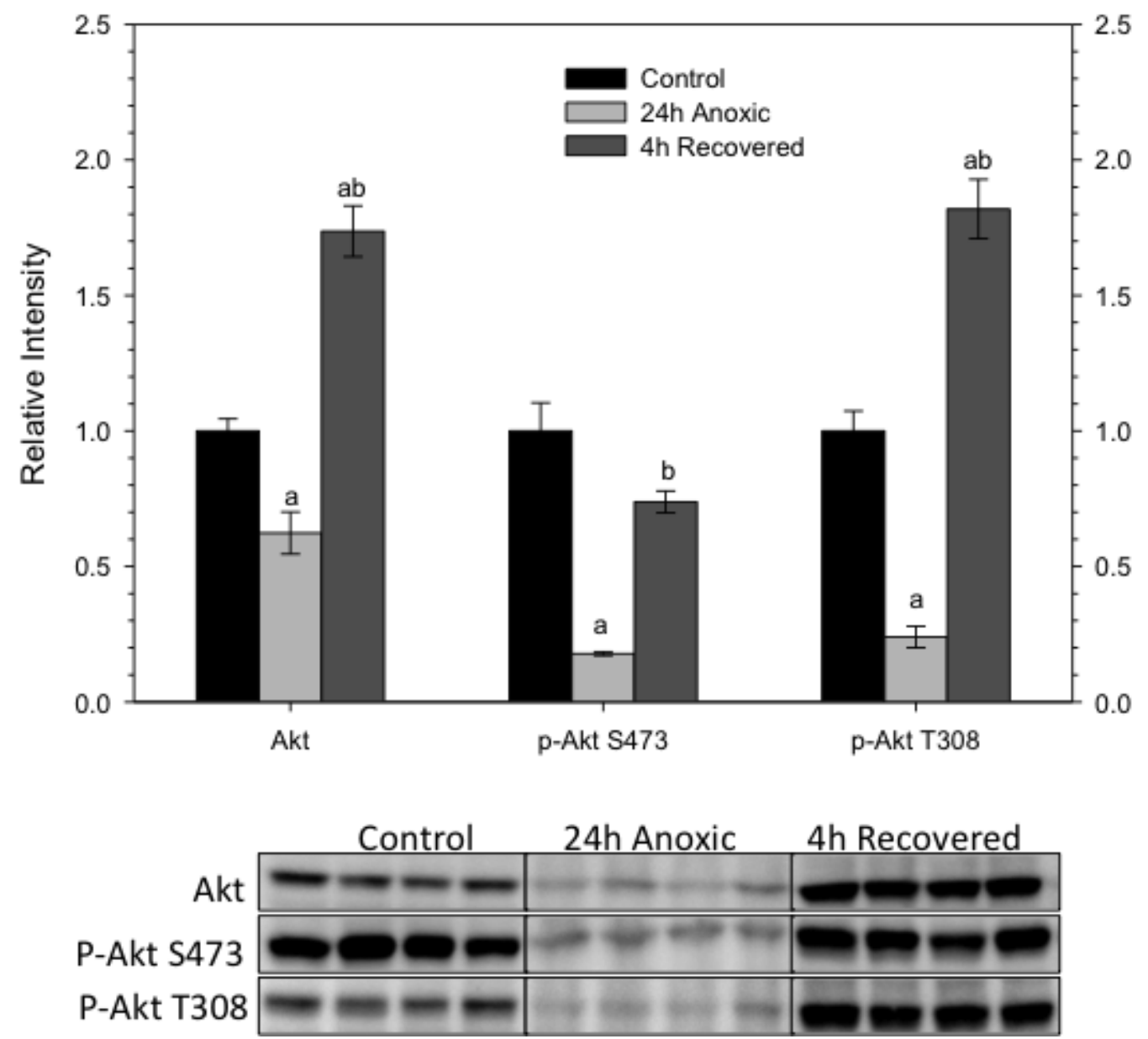
Fig. 4.3B
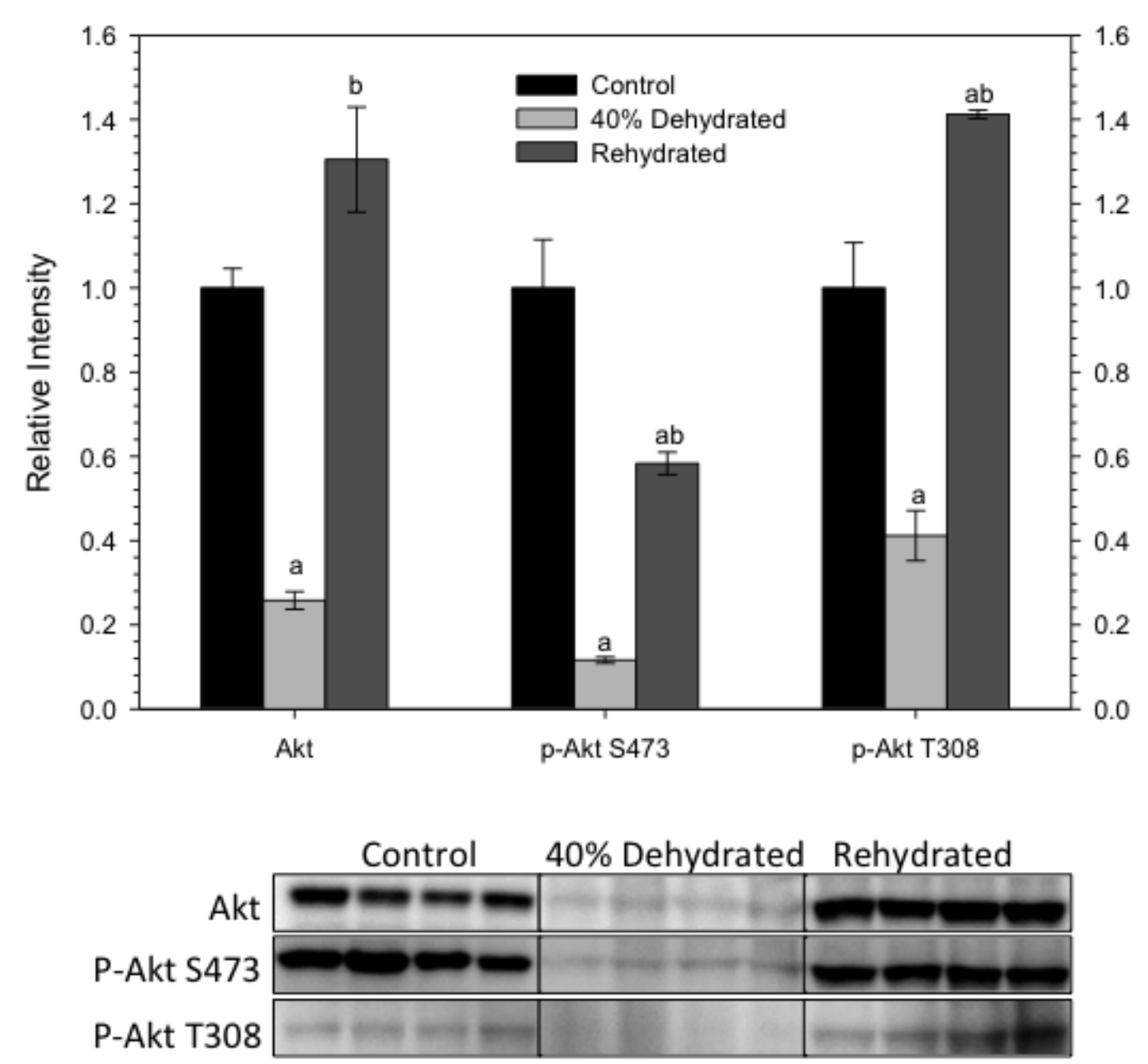

Fig. 4.3. Effects of $24 \mathrm{~h}$ anoxia and $40 \%$ dehydration on protein levels of Akt and relative contents of phospho-Akt Ser-473 and Thr-308 in wood frog liver. (A) $24 \mathrm{~h}$ anoxia exposure and $4 \mathrm{~h}$ recovery. (B) $40 \%$ dehydration and rehydration. a - Significantly different from the corresponding control $(\mathrm{P}<0.05)$; b- significantly different from the $24 \mathrm{~h}$ anoxic value (A) or 40\% dehydrated value (B). Other information as in Fig. 4.2. 
Fig. 4.4
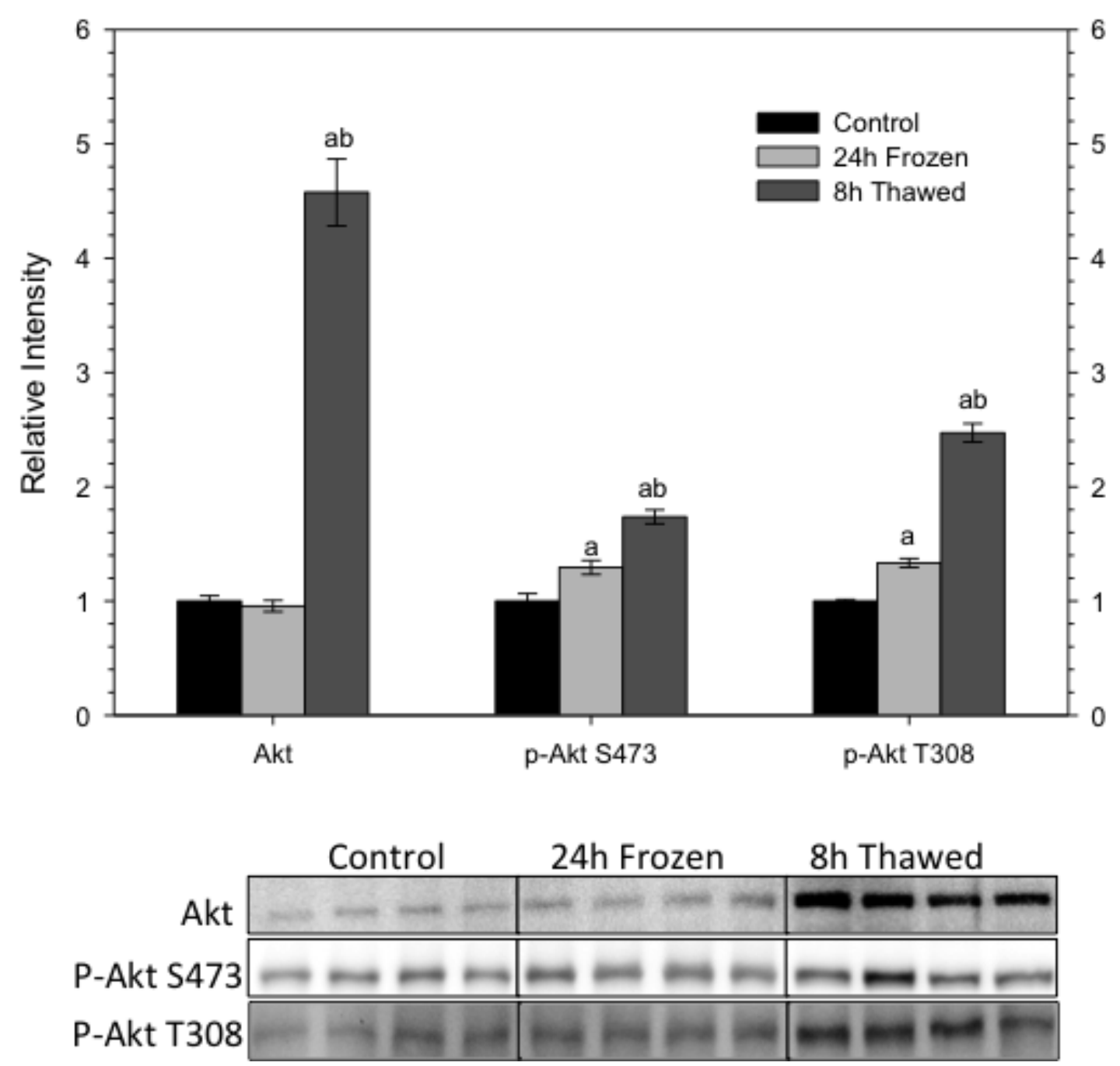

Fig. 4.4. Effects of $24 \mathrm{~h}$ freezing and $8 \mathrm{~h}$ thawing on the nuclear content of Akt protein and the relative levels of phospho-Akt Ser-473 and Thr-308 in wood frog liver. Other information as in Fig. 4.2. 
Fig. 4.5
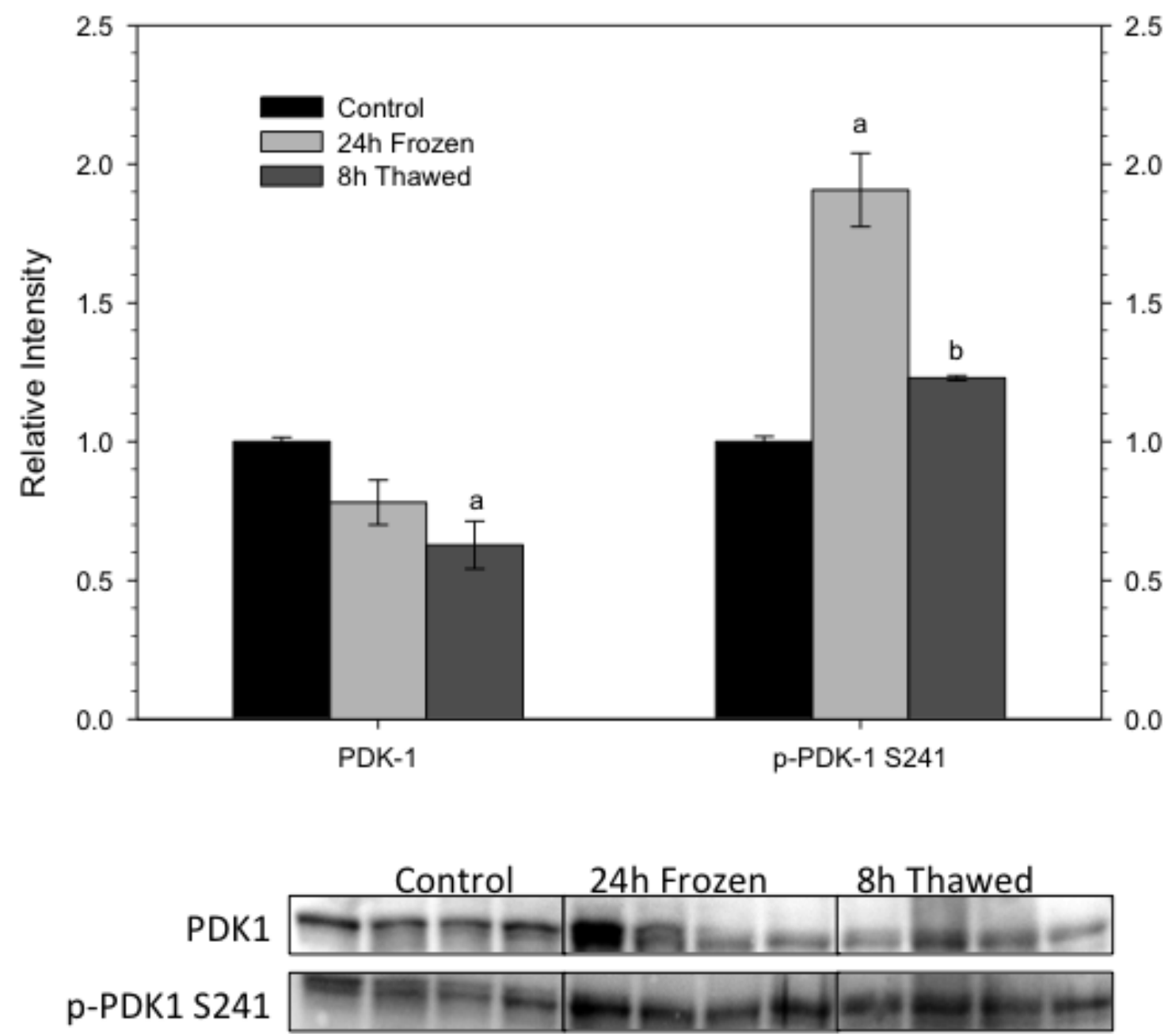

Fig. 4.5. Effects of $24 \mathrm{~h}$ freezing and $8 \mathrm{~h}$ thawing on protein levels of PDK-1 and relative phosphorylation of PDK-1 at Ser-241 in wood frog liver. Other information as in Fig. 4.2. 
Fig. 4.6A
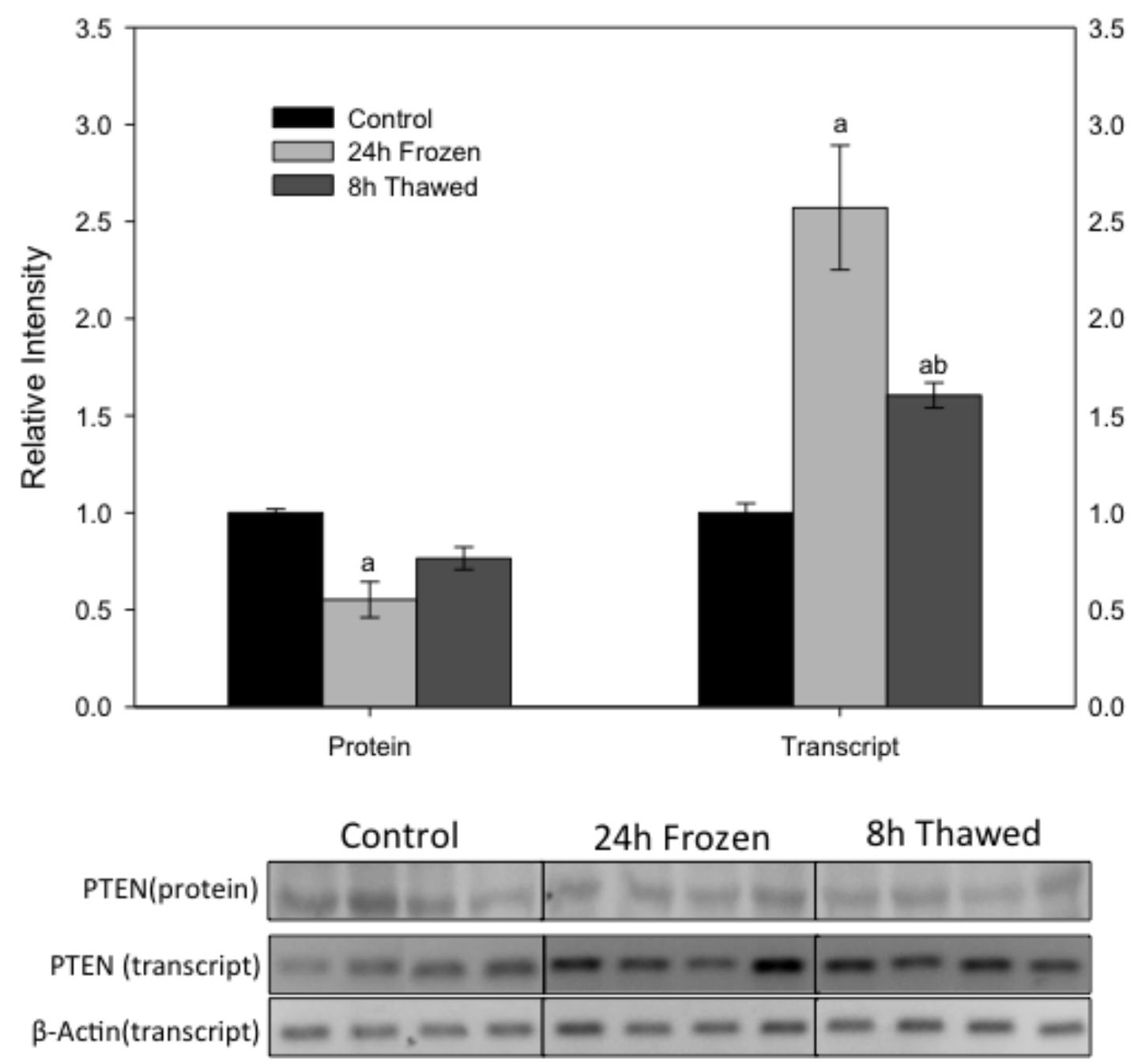
Fig. 4.6B
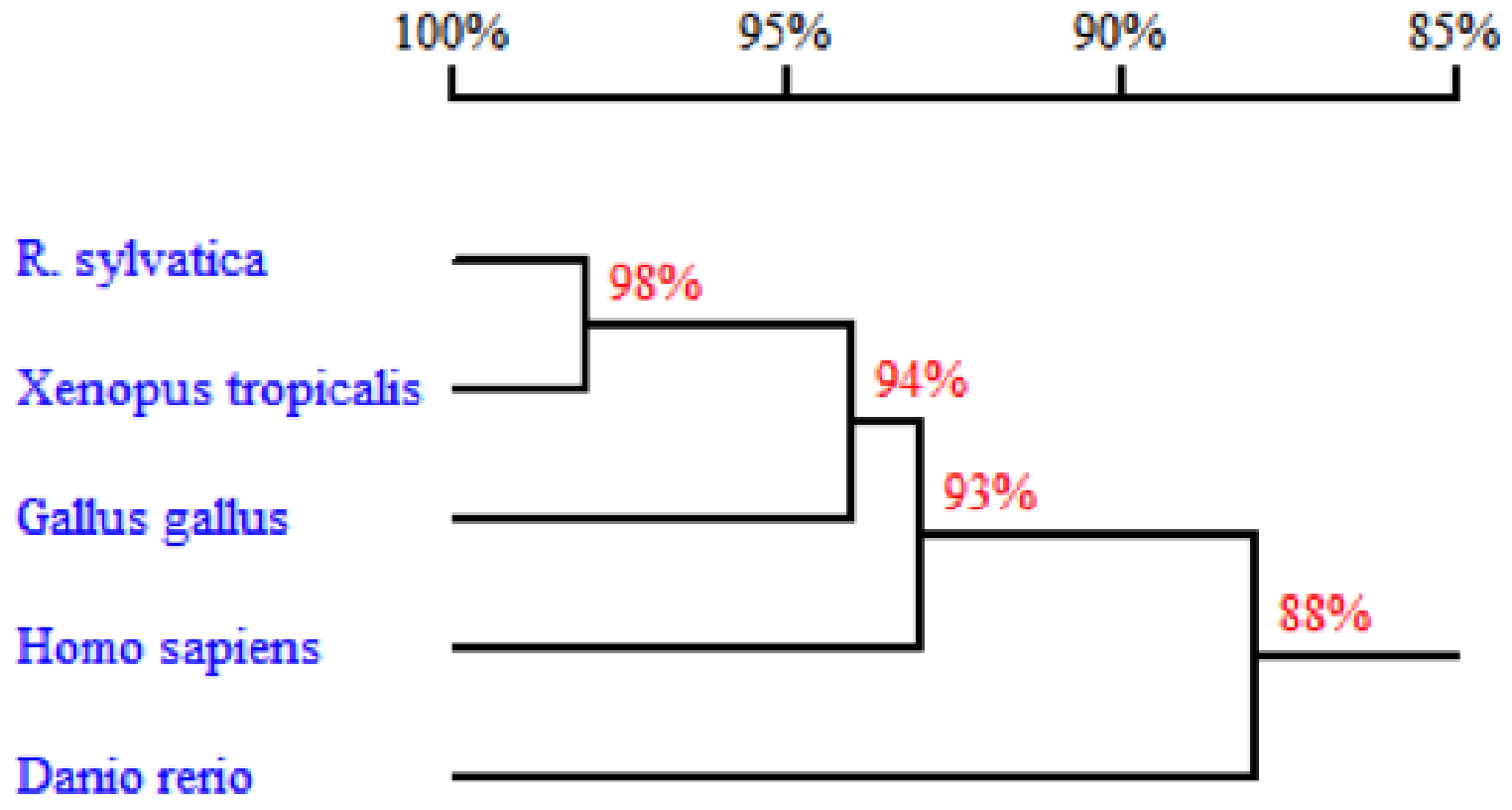

Fig. 4.6C

1. R.sylvatica

2. Xenopus tropicalis

3. Gallus gallus

4. Homo sapiens

5. Danio rerio

$\frac{1}{1} \frac{1}{M}$ TA I I KEXVSRNKRR YOE DG F DLDLTYI Y PN I I AMG F PAER LEGVYRNN

FDLDLTYI YPN I IAMG F PAER LEGVYRNN

MTA I IKEFVSRNKRR Y QEDG F DLDLTYI Y PN I I AMG F PAER LEGVYRNN

MTAIIKEIVSRNKRRYOEDG F DLDLTYI Y PN I I AMG F PAER LEGVYRNN

MTAI I K EIVSRNKRR YOEDG F DLDLTYI Y PN I IAMG F PAER LEGVYRNN MAA I I EFVSRNKRRYOEDG F DLDLTYI Y PNI IAMG F PAER LEGVYRNN

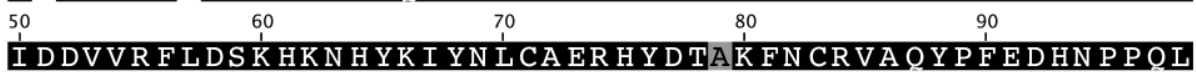

1. R.sylvatica

2. Xenopus tropicalis

3. Gallus gallus

4. Homo sapiens

5. Danio rerio

I DDVVR F LDSK HKN HYK I YN LCAER HYDTNK FS CRVA OY P F DHN P POL

I DDVVR F LDSK HKN H YK I YN LCAER HY DTN K F S CRVA QY P F E DHN P PQL

I DDVVR F LDSK HKN H YK I YN LCAER HY DTAK FN CRVA OY P FEDHN PPOL

I DDVVR F LDSK HKN H YK I YN LCA ER HY DTAK FN CRVA OY P F EDHN P POL

I D DVVR F LDSK HK D H YK I YN LCAER HY DTAK FN CRVA OY P F EDHN P POL

$100 \quad 110 \quad 120 \quad 130 \quad 140$

ELI K P F CEDLD ÓW LS EN DNHVAA I H CKA G K GR TGVM I CA YLLHRGK FLK

1. R.sylvatica

2. Xenopus tropicalis

3. Gallus gallus

4. Homo sapiens

5. Danio rerio

ELIK P F C E DLDOW LS EN DN-VAA I HCKAG K GR TGVM I CA Y LLHRGK FLK

ELIK P F CEDLDOW LSENEN-VAA I HCKAGKGR TGVM I CAYLLHRGKFPR

ELI K PF CEDLDOW LS ED DN HVAA I H CKA GKGR TGVM I CAYLLHRGK FLK

ELIK PFCEDLDOW LS ED DN HVAA I HCKA G K GR TGVM I CA Y LLHRGK FLK

ELI K P F CEDLDK W LS EN DN HVAA I H CKA G K GR TGVM I CA Y LLHRGK FKK

$\begin{array}{lllll}150 & 160 & 170 & 180 & 190\end{array}$

A OEA L DFYGEVR TR DKKGVTI PS ORR YVYYYS Y LLKNHLXYR PVALLFH

1. R.sylvatica

A QEALDFYGEVRTRD

2. Xenopus tropicalis

3. Gallus gallus

4. Homo sapiens

5. Danio rerio

A QEA LDF YG EVR TR DKK GV T I PS QRR YV YYYS Y LLKNNLEYR PVPLLFH

A QEA LDF Y GEVR TR DKK GV T I PS QRR YVYYYS Y LLKN HLDYR PVA LLF H

A OEA LDFYGEVR TR DKKGVTI PSORR YVYYXS YLLKNHLDYR PVALLFH

A OEA LDF YDEVR TR DK K GV T I PS ORR YVYYYS Y LLRNK LEYK PVALLFH 
Fig. 4.6. (A) Effects of $24 \mathrm{~h}$ freezing and $8 \mathrm{~h}$ thawing on PTEN in wood frog liver. Histogram shows mean relative PTEN protein and pten transcript levels under control, 24 $\mathrm{h}$ frozen and $8 \mathrm{~h}$ thawed conditions. Representative immunoblot bands for PTEN are shown below along with bands for pten and $\beta$-actin amplicons. Other information as in Fig. 4.2. (B) Homology tree for wood frog (R. sylvatica) PTEN partial amino acid sequence compared with western clawed frog (GenBank accession number: NM_001090362), chicken (Gallus gallus) (predicted sequence) (Genbank accession number: XP_421555.2), human (Homo sapiens) (Genbank accession number: ADM26757.1, PTEN like protein) and zebrafish (Danio rerio) (Genbank accession number: NP_001001822.1). Percentage similarities are given. (C) Partial amino acid sequence (141 residues) of wood frog PTEN aligned with partial PTEN (or PTEN-like) sequences of western clawed frog, chicken, human and zebrafish. The full length of the protein is approximately 400 amino acids. 
Fig. 4.7
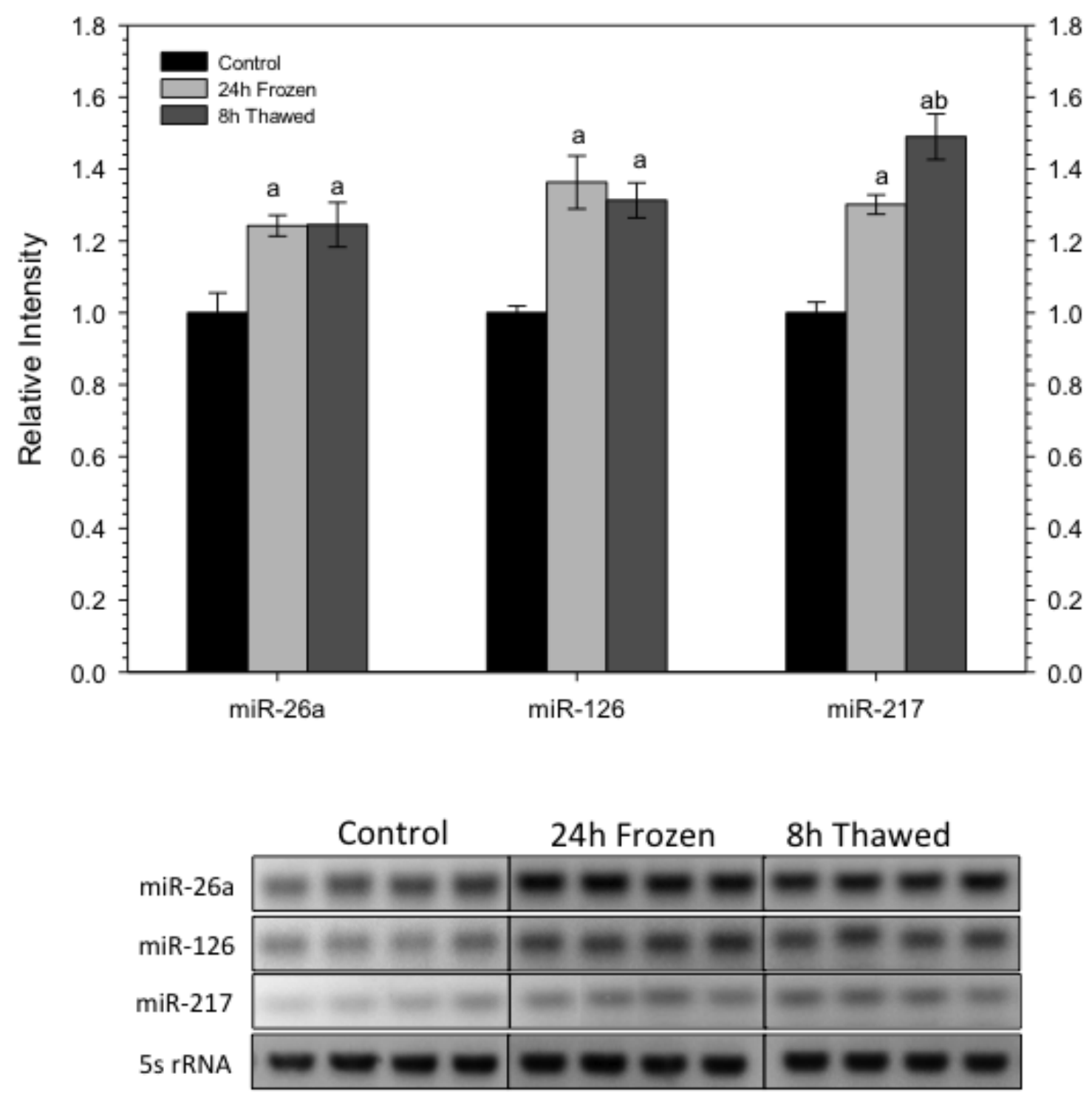

Fig. 4.7. Effects of $24 \mathrm{~h}$ freezing and $8 \mathrm{~h}$ thawing on transcript levels of miR-26a, miR126 and miR-217 in wood frog liver. Histogram shows standardized relative miRNA transcript levels. Lower panel shows representative RT-PCR bands as well as the corresponding bands for $5 \mathrm{~s}$ rRNA amplified from the same RNA samples. Other information as in Fig. 4.2 
Fig. 4.8
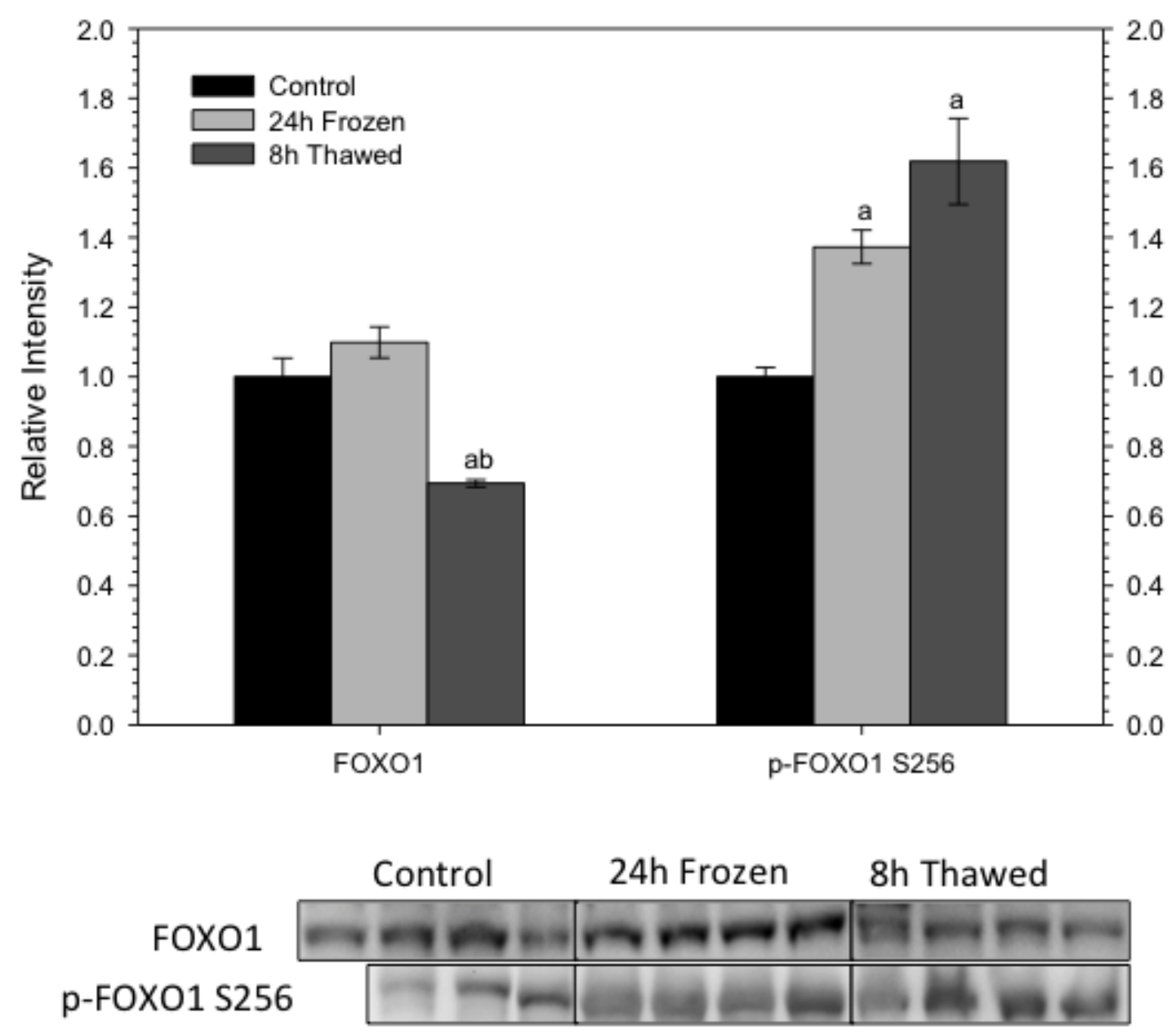

Fig. 4.8 Effects of $24 \mathrm{~h}$ freezing and $8 \mathrm{~h}$ thawing on total FOXO1 protein and phosphoFOXO1 Ser-256 levels in wood frog liver. Other information as in Fig. 4.2. 


\section{Chapter 5 Regulation and response of the TORC1 pathway}




\section{Introduction}

In order to balance ATP synthesis and consumption under stress conditions, optional energy expensive processes such as cell proliferation and cell growth need to be suppressed or inhibited. Protein synthesis is one of the key cellular events that are crucial to cell proliferation. However, it demands large amounts of energy and resources (Reeds et al., 1982), which is inconsistent the limited ATP production (glycolysis only) and fuel supply (carbohydrate only) available to wood frogs experiencing whole body freezing. Therefore, suppression of global protein synthesis is needed to strongly reduce protein synthesis and protein turnover in both proliferating and non-proliferating tissues, thereby helping to minimize energy expenditure in the frozen state. Although some freezespecific protein synthesis does occur (e.g. three novel proteins are specifically triggered in wood frog liver when freezing starts) (Storey and Storey, 2004a), in general, global protein synthesis must be suppressed. The current study hypothesizes that the TORC1 (target of rapamycin, complex 1) pathway is a critical target for suppression in wood frogs during whole body freezing, resulting from its role in directly promoting protein synthesis. This chapter explores the responses of key factors of TORC1 signaling in liver, muscle, heart and kidney of wood frogs in response to freeze-thaw cycles.

Crucial regulatory actions by $\mathrm{TORC} 1$ on protein synthesis have been well established over the years. TORC1 is a complex with kinase activity that consists of three core protein components: TOR, Raptor (regulatory associated protein of TOR) and G $\beta \mathrm{L}$ (G protein $\beta$-subunit-like protein, also known as mLST8). TOR provides the kinase activity for the complex, whereas Raptor and G $\beta$ L serve more regulatory roles for maintaining the structure of the complex. TORC1 regulates protein synthesis through 
several phosphorylation events on its downstream targets, which are mainly involved in the mRNA translation initiation process (Hay and Sonenberg, 2004). Raptor directs the specific interaction of the complex with downstream targets by binding to the TOS motif that is present in the primary structure of target proteins (Schalm et al., 2004). G $\beta \mathrm{L}$ functions as a positive regulator of TORC1 kinase activity via its interaction with the catalytic domain of TOR, and is sensitive to extracellular environmental changes, e.g. nutrient availability (Kim et al., 2003). It was also proposed that G $\beta$ L stabilizes TORC1 association (Kim et al., 2003). Accordingly, the current understanding of TORC1 is that TOR provides the kinase activity, G $\beta$ L responds to extracellular signaling as well as stabilizes the complex, and Raptor reaches downstream to ensure target specificity of the complex.

TORC1 promotes mRNA translation initiation, thereby stimulating protein synthesis in the cell and regulation of $\mathrm{TORC} 1$ is responsive to conditions that may alter metabolic status. mRNA translation initiation is a multi-step process that comprises a series of complex assembly and disassembly processes (Hershey and Merrick, 2000). TORC1 regulation of pre-initiation complex assembly is critical to mRNA translation. TORC1 mainly regulates the translation of mature mRNAs with 5' cap structure (Hay and Sonenberg, 2004). One critical step in translation initiation is the assembly of the eIF4F (eukaryotic translation initiation factor 4F) complex which consists of eIF4E, eIF4G and eIF4A. eIF4E first binds to the 5' cap structure of an mRNA transcript and this recruits the rest of the initiation factors to form the complete eIF4F complex (Hershey and Merrick, 2000). Thus, eIF4E is a crucial to eIF4F assembly. The interaction between eIF4E and the 5'cap structure of mRNA is subject to inhibitory 
regulation from 4EBP (eIF4E binding protein) which, by binding eIF4E, blocks the interaction between eIF4E and eIF4G, thereby inhibiting eIF4F assembly (Gingra et al., 1999). TORC1 directly regulates 4EBP via phosphorylation. Hyper-phosphorylated 4EBP dissociates from the eIF4E-4EBP complex, enabling assembly of the eIF4F complex (Gingra et al., 1999; Hershey and Merrick, 2000) (Fig. 5.1A). In addition, some mature mRNAs contain regulatory elements in their 5'UTR ( $5^{\prime}$, untranslated region) that form inhibitory secondary structures, preventing the pre-initiation complex from passing through (Manzella et al., 1991). Another major downstream target of TORC1, S6K (40S ribosomal protein S6 kinase) plays a critical role in such cases (Isotani et al., 1999; Nojima et al., 2003). After being phosphorylated by TORC1, the active S6K phosphorylates and activates eIF4B, which in turn binds to eIF4G of the eIF4F complex and subsequently enhances the RNA helicase activity of eIF4A in the complex. This removes the inhibitory secondary RNA structure allowing the pre-initiation complex to scan through and approach the starting codon (Shahbazian et al., 2006) (Fig. 5.1B).

Upstream inhibitors and multiple phosphorylation events directly regulate TORC1 assembly and activity. Activation of TORC1 kinase activity is dependent on GTP-RHEB (GTP bound Ras homologue enriched in brain) (Ma and Blenis, 2009). TSC1/TSC2 (tuberous sclerosis 1 and 2) complex is one of the major inhibitors of TORC1 due to its roles in regulating GTP-RHEB. GTP-RHEB is converted to its inactive form, GDPRHEB, by the active TSC1/TSC2 complex via the GAP (GTPase-activating protein) activity of TSC2, thereby inhibiting the phosphotransferase activity of TORC1 (Castro et al., 2003). PRAS40 (proline-rich Akt substrate $40 \mathrm{kDa}$ ) is another key TORC1 inhibitor that binds to the kinase domain of TOR in response to environmental stresses such as 
nutrient deprivation to inhibit kinase activity (Vander Haar et al., 2007). Regulation on these inhibitors and the core components of TORC 1 are heavily dependent on protein phosphorylation, which is conducted by kinase signaling pathways including the Akt/PKB pathway. TSC2 can be phosphorylated by Akt at Ser-939, which enables a binding site for 14-3-3 proteins on it that disrupts its interaction with RHEB, thereby preventing TSC1/TSC2 from deactivating GTP-RHEB (Cai et al., 2006). Therefore, the phosphorylation state of TSC2 can be considered as an indicator of TSC1/TSC2 function. PRAS40 is also subject to regulation by the Akt pathway. Akt-dependent Thr-246 phosphorylation of PRAS40 leads to its dissociation from TORC1 in a 14-3-3 proteindependent manner, thereby relieving its inhibitory effect on TORC1 (Vander Haar et al., 2007; Wang et al., 2007).

Both Raptor and TOR within TORC1 are regulated by phosphorylation. Raptor is subject to phosphorylation regulation from multiple kinase signaling pathways. For example, it can be regulated by AMPK-dependent phosphorylation (Gwinn et al., 2008). Phosphorylated Raptor at Ser-863 plays critical roles in enhancing TORC1 activity through a positive feedback mechanism in which activated TORC1 promotes Ser-863 phosphorylation on Raptor, which in turn promotes the kinase activity of the complex (Wang et al., 2009; Foster et al., 2010). In terms of TOR, the protein can be autophosphorylated at Ser-2481 (Paterson et al., 2000) and phosphorylated at Ser-2448 by S6K (Chiang and Abraham, 2005). It has been proposed that Ser-2481 phosphorylation usually occurs in the alternate TOR complex 2 (TORC2) whereas Ser2448 phosphorylated TOR is found in TORC1 (Copp et al., 2009), making both phosphorylation events markers of TOR complex assembly. 
Overall, TOR, Raptor, TSC2 and PRAS40 (with their phosphorylated forms) are critical to TORC1 regulation. The current study investigates total and phosphorylation levels of TOR and Raptor, protein levels of G $\beta$ L as well as levels of phosphorylated TSC2 and PRAS40 in response to whole body freezing of wood frogs. In addition, immunoprecipitation was used to monitor TORC1 assembly in wood frogs under freezing stress. Furthermore, cellular levels of total and TORC1-dependent phosphorylated 4EBP and $\mathrm{S} 6 \mathrm{~K}$ were examined to confirm the downstream effects from TORC1 pathway regulation in response to freeze-thaw.

\section{Results}

Relative protein levels of TOR and phospho-TOR Ser-2448 in wood frogs responding to freezing stress

Relative protein levels of TOR and the phosphorylation state of TOR Ser-2448 were assessed using immunoblotting in liver, muscle, heart and kidney of control $\left(5^{\circ} \mathrm{C}\right.$ acclimated), $24 \mathrm{~h}$ frozen (at $-2.5^{\circ} \mathrm{C}$ ) and $8 \mathrm{~h}$ thawed (at $5^{\circ} \mathrm{C}$ ) wood frogs. Polyclonal mammalian antibodies used to detect TOR and phospho-TOR Ser-2448 cross-reacted with a single band at $\sim 290 \mathrm{kDa}$ in wood frog tissue extracts that corresponded to the molecular weight reported in other species. Fig. 5.2A shows that 24 freezing led to significant decreases in relative total TOR protein levels in wood frog liver, muscle and heart, decreasing to levels that were $51.95 \pm 31 \%, 49.88 \pm 6.1 \%$ and $69.88 \pm 1.57 \%$ of the corresponding control values, respectively. However, relative total protein level of TOR in kidney increased $1.45 \pm 0.08$-fold compared to the control value after $24 \mathrm{~h}$ freezing exposure. After an $8 \mathrm{~h}$ thaw, total TOR levels in heart and kidney were restored to control 
levels. In liver and muscle, however, TOR levels also rose and reached values that were significantly greater than controls, $1.36 \pm 0.1$-fold and $1.39 \pm 0.09$-fold higher, respectively.

Fig. 5.2B shows the relative phosphorylation state of TOR Ser-2448 in the four tissues in response to freeze/thaw cycle. No appreciable change in phospho-TOR Ser2448 content occurred in liver or heart after $24 \mathrm{~h}$ freezing exposure; whereas muscle and kidney exhibited decreases in the phospho-TOR Ser-2448 content to $42.6 \pm 3.18 \%$ and $67.6 \pm 5.4 \%$ of the corresponding control values, respectively. After an $8 \mathrm{~h}$ thaw, phospho-TOR Ser-2448 levels in liver increased strongly by $1.96 \pm 5.06$-fold compared to controls. However, heart phospho-TOR Ser-2448 levels dropped significantly to $53.3 \pm$ $7.7 \%$ of control values and phospho-TOR Ser-2448 content remained low in muscle and kidney; levels were $36.5 \pm 0.9 \%$ and $54.1 \pm 7.7 \%$ of the corresponding control values, respectively.

Relative protein levels of Raptor and phospho-Raptor Ser-863 in wood frog tissues responding to freezing stress

Relative protein levels of Raptor and phospho-Raptor Ser-863 were assessed in liver, muscle, heart and kidney from control, $24 \mathrm{~h}$ frozen and $8 \mathrm{~h}$ thawed frogs using immunoblotting. Polyclonal mammalian antibodies cross-reacted with a single band at $\sim 150 \mathrm{kDa}$ corresponding to the molecular weight of Raptor in the other species. As shown in Fig. 5.3A, relative protein levels of Raptor were unchanged in all tissues during freezing and also remained unchanged after thawing in muscle and heart. However, relative Raptor level decreased to $60.6 \pm 3.3 \%$ and $51.9 \pm 5.2 \%$ of control values in liver 
and kidney after $8 \mathrm{~h}$ thawed, respectively. Fig. 5.3B shows the effect of freeze/thaw on the phosphorylation state of Raptor Ser-863. Relative phospho-Raptor S863 content did not change in any of the four tissues after $24 \mathrm{~h}$ freezing exposure. However, $8 \mathrm{~h}$ thawing increased phospho-Raptor Ser-863 levels in liver and muscle by $1.7 \pm 0.17$-fold and 1.49 \pm 0.1 -fold compared with control values, respectively.

Relative protein level of G $\beta L$ in wood frogs responding to freezing stress

The effect of the freeze-thaw on the relative expression levels of G $\beta \mathrm{L}$ protein was also assessed in the four wood frog organs. A single band at $\sim 35 \mathrm{kDa}$ corresponding to the known molecular weight in other species was detected by a polyclonal mammalian antibody. Fig. 5.4 shows that during freezing the relative protein levels of G $\beta \mathrm{L}$ dropped significantly to $69.7 \pm 2.3 \%$ and $87.4 \pm 4.2 \%$ of the corresponding control values in skeletal muscle and heart, respectively, whereas liver and kidney showed no appreciable change in G $\beta \mathrm{L}$ protein level after 24h freezing. However, liver showed elevated G $\beta \mathrm{L}$ protein upon $8 \mathrm{~h}$ thaw with a fold change of $1.35 \pm 0.06$ as compared with controls. G $\beta \mathrm{L}$ content was restored to control levels in $8 \mathrm{~h}$ thawed muscle but decreased to $62.5 \pm 2.2 \%$ and $67.9 \pm 5 \%$ of the corresponding control values in heart and kidney, respectively, of thawed animals.

Relative protein levels of $4 E B P$ and phospho-4EBP Thr-45 in wood frogs responding to freezing stress 
Relative protein levels of 4EBP and phospho-4EBP Thr-45 were assessed in frog organs using polyclonal mammalian antibodies that cross-reacted with a single band at $\sim 18 \mathrm{kDa}$ corresponding to the known molecular weight of 4EBP in other species. Fig. 5.5A shows that after $24 \mathrm{~h}$ freezing, relative protein levels of total 4EBP dropped significantly in liver, heart and kidney to values that were $46.5 \pm 6.3 \%, 24.79 \pm 2.4 \%$ and $31.76 \pm 2.2 \%$ of the corresponding control values, respectively. Upon $8 \mathrm{~h}$ thaw, total 4EBP levels increased again reaching high levels in liver $(1.48 \pm 0.09$-fold higher than controls) and rising to $52.2 \pm 2.4 \%$ and $54.4 \pm 10.4 \%$ of the corresponding control levels in heart and kidney, respectively. Skeletal muscle 4EBP was unaffected by freeze-thaw.

The relative phosphorylation state of 4EBP Thr-45 decreased strongly in all four tissues in response to $24 \mathrm{~h}$ freezing exposure. As shown in Fig. 5.5B, relative phospho4EBP Thr-45 content decreased to $25.7 \pm 5.6 \%, 28.8 \pm 2.8 \%, 39.6 \pm 5 \%$, and $25.8 \pm 2.5 \%$ in liver, muscle, heart and kidney as compared with corresponding controls, respectively. However, after an $8 \mathrm{~h}$ thaw phospho-4EBP Thr-45 levels recovered partly or fully in all four tissues. Phospho-4EBP Thr-45 content was restored to $\sim 1.3$-fold higher than controls in liver and increased by $1.38 \pm 0.07$-fold in kidney as compared to controls. In muscle and heart phospho-4EBP Thr-45 content rose significantly over $24 \mathrm{~h}$ frozen values but still remained significantly lower at $57.2 \pm 2.5 \%$ in muscle and $58.9 \pm 4.6 \%$ in heart of the corresponding control values.

Relative protein levels of S6K and phospho-S6K Thr-389 in wood frog tissues responding to freezing stress 
Polyclonal antibodies for S6K and phospho-S6K Thr-389 cross-reacted with a single band at $\sim 70 \mathrm{kDa}$ corresponding to the known molecular weight of S6K reported in other vertebrates. Fig. 5.6 shows the effects of freeze/thaw on protein levels of total and phosphorylated (Thr-389) S6K. Upon 24h freezing exposure, relative S6K protein levels dropped significantly in liver and muscle to $47.8 \pm 8 \%$ and $59.0 \pm 7.1 \%$ of the corresponding control values, respectively (Fig. 5.6A). S6K levels were restored to control levels in liver after $8 \mathrm{~h}$ thawed but remained low in muscle at $23.2 \pm 2.2 \%$ of the control value. However, S6K protein levels did not change in heart or kidney over the freeze/thaw cycle.

Fig. 5.6B shows the relative phosphorylation state of S6K Thr-389 in the four tissues. Freezing reduced phospho-S6K Thr-389 content in liver, muscle and kidney; the values were $37.6 \pm 2.5 \%, 81.6 \pm 4$ and $64.4 \pm 1.8 \%$ of the corresponding control levels, respectively. Phospho-SK6 Thr-389 content restored to control values after an $8 \mathrm{~h}$ thaw in wood frog liver and muscle but increased significantly by $1.17 \pm 0.03$-fold as compared to controls in kidney. Heart phospho-S6K Thr-389 content did not change appreciably in response to freeze /thaw.

Relative phosphorylation state of phospho-TSC2 Ser-939 and phospho-PRAS40 Thr-246 in wood frog responding to freezing stress

Relative phospho-TSC Ser-939 and phospho-PRAS40 Thr-246 contents were assessed over freeze-thaw in wood frog liver, muscle, heart and kidney. Polyclonal mammalian antibodies cross-reacted with single bands at $\sim 250 \mathrm{kDa}$ for TSC2 and $\sim 40 \mathrm{kDa}$ for PRAS40, the known molecular weights from other vertebrates. Relative 
phospho-TSC2 Ser-939 levels increased significantly in liver by $1.29 \pm 0.05$-fold after $24 \mathrm{~h}$ freezing whereas phosphoprotein content in muscle and heart decreased to $50.8 \pm$ $2.3 \%$ and $59.9 \pm 7.5 \%$ of the corresponding control levels and levels did not change in kidney (Fig. 5.7A). Upon 8 thawing, liver showed a decrease in phospho-TSC2 Ser-939 levels to a value of $57.7 \pm 1.9 \%$ of the control level and muscle levels fell further to 35.3 $\pm 3.8 \%$ of the control value. Opposite responses were seen in heart and kidney; phosphoTSC2 Ser-939 content was restored to control values in heart, whereas kidney showed a significant increase in phosphoprotein content of $1.99 \pm 0.32$-fold after thawing.

Fig. 5.7B shows the effects of freeze/thaw cycle on phospho-PRAS40 Thr-246 levels. All four tissues showed no appreciable changes in phospho-PRAS40 Thr-246 content in response to $24 \mathrm{~h}$ freezing. However, the $8 \mathrm{~h}$ thaw resulted in significant increases in PRAS40 phosphorylation state liver and muscle with fold increases of $1.73 \pm$ 0.068 and $1.63 \pm 0.14$. By contrast, relative phospho-PRAS40 Thr-246 levels in heart dropped to $39.7 \pm 9.25 \%$ of control values whereas levels did not change in kidney.

\section{Effects of freezing stress and recovery on TORC1 complex assembly}

TORC1 complex assembly status in response to freeze-thaw was investigated in wood frog tissues using the technique of co-immunoprecipitation (CO-IP). Fig. 5.8 shows immunoblot images from CO-IP trials using Raptor antibody as the pull down antibody and P-TOR (Ser-2448) as the detection antibody. Immunoblots probed with primary Raptor antibody showed that protein was successfully pulled down in all four tissues. The intensities of P-TOR (Ser-2448) bands were compared qualitatively. Liver showed stronger phospho-TOR (Ser-2448) signal in control and thawed frogs than in the frozen 
group. Apart from a weak signal for phospho-TOR (Ser-2448) detected in liver from frozen frogs, phospho-TOR (Ser-2448) signal was absent from muscle, heart and kidney of the frogs after 24h freezing. However, the signal of P-TOR (Ser-2448) re-appeared in all tissues from $8 \mathrm{~h}$ thawed animals.

\section{Discussion}

There is evidence of regulated metabolic rate depression in wood frogs during freezing exposure (Sinclair et al., 2013). Therefore, as in other hypometabolic systems, it appears that coordinated changes in energy expenditure are required for wood frogs to survive whole body freezing involving global repression of many metabolic functions along with selected specific responses. In fact, multiple studies have explored the activation of specific genes and signaling pathways involved in protecting cells and tissues from freezing, anoxia and dehydration in wood frog (Storey and Storey, 1984; Storey, 1987a; Churchill and Storey, 1993, 1994; Holden and Storey, 1997; Storey and Storey, 2001). With limited energy output during hypometabolism under freezing exposure, ATP-expensive biological processes are suppressed in order for the frogs to reprioritize energy expenditure to such stress responsive adaptations. Studies reported in this thesis demonstrate that the cell cycle is one of the processes that is strongly suppressed in wood frogs during freezing exposure (Chapter 3). Protein synthesis is another well-documented cellular process that demands substantial energy input to proceed (Reeds et al., 1982; Neijssel et al., 1996). It has also been reported that some species such as the Antarctic sea urchin (Sterechinus neumayeri) maintain their rate of protein biosynthesis during cold exposure, as one of their adaptations (Pace and 
Manahan, 2007). Therefore, regulation of protein synthesis could be an important marker leading to species-specific stress response in wood frogs when experiencing freeze-thaw cycles. TORC1-dependent protein synthesis holds an especially important place as a protein synthesis fingerprint in wood frog not only because it is one major mRNA translation regulator, but also a downstream target to major cell signaling pathways (i.e. Akt pathway). The TORC1 response also reflects energy status due to the GTP-dependent nature of its function.

The present study examined the relative protein expression and phosphorylation status of TORC1 pathway core components in liver, muscle, heart and kidney of wood frogs upon exposure to freeze-thaw conditions. The results showed that the protein and phosphorylation levels of most TORC1 components were suppressed in response to freezing in all four tissues of wood frogs, followed by a recovery response after $8 \mathrm{~h}$ thaw (Fig. 5.2-5.4). However, some tissue specific patterns were observed. For example, the TOR protein level dropped significantly in liver, muscle and heart after $24 \mathrm{~h}$ freezing exposure as compared with control animals but only muscle exhibited an accompanying decrease in the amount of active phosphorylated TOR (at Ser-2448) (Fig. 5.2). Also, the level of inhibition of TORC1 components in response to freezing stress varied among tissues. Upon freezing exposure, liver exhibited inhibition only of TOR protein levels (by $\sim 50 \%$ ), with no appreciable signs of regulation of other TORC1 components (Raptor, G $\beta$ L) (Fig. 5.2-5.4). Heart showed reduced protein expression levels of both TOR and G $\beta L$ that dropped significantly to around $70 \%$ and $85 \%$ of the control values, respectively (Fig. 5.2A, 5.4). Muscle showed the strongest inhibitory effects on expression and posttranslational levels of TORC1 components with reduced levels of 
both protein and phosphorylation state of TOR, as well as G $\beta$ L protein (Fig 5.2-5.4). However, kidney, while showing signs of TORC1 inhibition during freezing exposure due to a decreased phosphorylation state of TOR (around 65\% of the control level), showed an increase in total TOR protein. The kidney increase of TOR protein level might be associated with glucose accumulation in wood frogs when experiencing cold (Fig. 5.2). This agrees with the phenomenon observed by Zhang et al. (2012) in mammalian cell culture where high glucose content stimulates expression of TOR. A decrease in phospho-TOR (Ser-2448) in muscle and kidney may also be the result of inhibition of the direct upstream kinase S6K (see below) as well as a possible blockage of upstream signal transduction via Akt. Interestingly, 24h freezing failed to introduce appreciable changes in either protein or phosphorylation levels of Raptor in any of the tissues examined, indicating that regulation of TORC1 during freezing exposure may be independent of Raptor (Fig. 5.3).

Apart from a universal recovery of TOR protein levels in all tissues during thawing (Fig. 5.2A), tissue specific regulation of the expression and phosphorylation levels of TORC1 components were observed after $8 \mathrm{~h}$ thawing. Indeed, TOR protein levels actually increased significantly by $\sim 1.4$-fold over controls in liver and muscle (Fig. 5.2A), suggesting not only recovery but a stimulation of protein synthesis. In liver, the phosphorylation state of TOR also increased strongly (almost 2-fold) during thawing and increased phosphorylation of Raptor (Ser-863) and increased amounts of G $\beta L$ were also observed (Fig. 5.2B, 5.3B, 5.4). These results strongly suggest that TORC1 is activated upon thawing in liver. Similarly, skeletal muscle exhibited increased levels of TOR, phospho-Raptor (Ser-863) as well as a recovered G $\beta$ L level, also suggesting a probable 
activation or recovery of TORC1 assembly/function after thawing (Fig. 5.2A, 5.3B, 5.4). Interestingly, however, the phosphorylation state of TOR (Ser-2448) remained inhibited after $8 \mathrm{~h}$ thaw in muscle (Fig. 5.2B). The phosphorylation status of TOR at Ser-2448 is largely dependent on the activity of S6K and upstream Akt signaling. Therefore, the low phospho-TOR (Ser-2448) level may be a result of the strong suppression of S6K levels in muscle of $8 \mathrm{~h}$ thawed frogs (see below). In heart, protein levels and phosphorylation state of TOR showed a similar pattern as in skeletal muscle during freeze/thaw whereas no appreciable changes were observed in Raptor protein and phosphorylation levels (Fig. 5.2, 5.3). G $\beta L$ protein levels, however, dropped to $\sim 60 \%$ of the control level during thawing in heart (Fig. 5.4). Kidney exhibited the most interesting effect of thawing among the tissues. Despite a recovery of the TOR protein level, signs of inhibition were observed for all other TORC1 factors, including decreased levels of phospho-TOR (Ser-2448) as well as Raptor and $\mathrm{G} \beta \mathrm{L}$ protein (Fig. 5.2B, 5.3A, 5.4).

Based on the general trend of inhibition and recovery of the protein and phosphorylation levels of TORC1 components, the technique of CO-IP was used to further investigate actual TORC1 assembly. A primary antibody specific to Raptor was used to pull down the complex. Phospho-TOR (Ser-2448) was then assessed in the precipitated samples, due to the fact that this phosphorylated form represents active TORC1 (Copp et al., 2009). After successfully pulling down Raptor (confirmed by immunoblotting using the Raptor antibody, Fig. 5.8), the presence of phospho-TOR (Ser2448) was assessed in the immunoprecipitated samples from all four tissues. Firstly, the results showed that the band intensities of Raptor generally matched the protein levels observed from immunoblotting. Phospho-TOR (Ser-2448) was detected in all four tissues 
of control frogs. As expected, the intensity of the phospho-TOR (Ser-2448) band was either significantly weaker (liver) or completely absent (muscle, heart and kidney) from the frogs after 24h freezing (Fig. 5.8), indicating a strongly reduced phospho-TOR (Ser2448): Raptor interaction, which indicates inhibition of the active TORC1 complex. Upon thawing, the phospho-TOR (Ser-2448) re-appeared in tissues, indicating recovery of the phospho-TOR: Raptor interaction, ie. a potential restoration of TORC1 assembly. The results from CO-IP provide strong evidence that TORC1 assembly was inhibited in all four tissues of frozen frogs but recovered again upon $8 \mathrm{~h}$ thaw. This suggests probable suppression of mRNA translation during freezing and a recovery after thawing.

In order to assess the downstream effects of TORC1 inhibition during freezing, levels of 4EBP and S6K proteins were evaluated along with the phosphorylation state of residues targeted by TORC1. The protein level of 4EBP dropped by more than $50 \%$ in liver, heart and kidney after $24 \mathrm{~h}$ freezing exposure (compared with controls) and was accompanied by universal decreases in phosphorylation status (Thr-45) in all tissues (Fig. 5.5). In terms of $\mathrm{S} 6 \mathrm{~K}$, the protein levels in liver and muscle decreased to around $50 \%$ of the control levels during freezing whereas no appreciable changes were observed in heart and kidney (Fig. 5.6A). The phosphorylation level of S6K (Thr-389) dropped significantly in liver, muscle and kidney while no change was seen in heart (Fig. 5.6B). These results indicate that the TORC1-dependent phosphorylation states of S6K responded according to the suppression of the complex assembly observed from the COIP results in liver, muscle and kidney. Therefore, the freeze responsive patterns of both 4EBP and S6K generally matched with the results of the expression and phosphorylation 
levels of the core complex components, as well as the changes observed in the phosphoTOR:Raptor interaction.

4EBP plays critical roles in mRNA translation initiation. As mentioned previously, the TORC1-dependent phosphorylation of 4EBP interrupts the 4EBP:eIF4E interaction, thereby promoting the formation of the translation pre-initiation complex that is required for mRNA translation initiation (Hay and Sonenberg, 2004). S6K also holds a pivotal place in stimulating mRNA translation. Phosphorylated S6K activates eIF4B, which in turn interacts with eIF4G and eIF4A to promote the assembly of the translation preinitiation complex (Isotani et al., 1999; Nojima et al., 2003). With the drop in both phospho-4EBP and phospho-S6K in liver, muscle and kidney, the TORC1-dependent initiation of mRNA translation appeared to be blocked in these tissues in response to freezing stress. Notably, in heart of frozen frogs, the downstream effect from the decreased TORC1 complex level was only reflected through the reduced phosphorylation state of 4EBP but not S6K. The unaltered S6K phosphorylation status in heart may result from alternative mechanisms. Indeed, it has been demonstrated that $\mathrm{S} 6 \mathrm{~K}$ can be activated in an TOR-independent fashion (Lehman et al., 2007). Furthermore, the observed S6K protein and phosphorylation levels in heart were consistent with the phospho-TOR (Ser2448) level, given that S6K is directly responsible for this phosphorylation event on TOR (Fig. 5.2B, 5.6B) (Chiang and Abraham, 2005). In fact, heart is one of last tissues to freeze in wood frog due to its high glucose content and its crucial role in distributing cryoprotectant for as long as possible (Rubinsky et al., 1994). Therefore, it is not a surprise that the heart was more active than other tissues during freezing exposure. However, the drop of phospho-S6K level in liver did not trigger a corresponding change 
in phospho-TOR (Ser-2448) level. This may be a result of the previously demonstrated Akt activation in liver of frozen frogs (Chapter 4), since this pathway was proposed to play a positive role in Ser-2448 phosphorylation of TOR (S k ić et al., 2000). It is quite possible that the activation of Akt and inhibition of phosphorylation on S6K negate each other's regulatory effect on TOR Ser-2448 phosphorylation. The phenomena may also suggest a coordinated Akt signal transduction flow to its various downstream targets that favors cell preservation responses and survival but not energy-costly protein synthesis, which matches the orchestrated energy expenditure during hypometabolism.

Upon $8 \mathrm{~h}$ thaw, both the protein and phosphorylation levels of 4EBP and S6K recovered in liver, consistent with the immunoblotting and CO-IP results of TORC1 (Fig. 5.2-5.4, 5.5, 5.6, 5.8). In muscle, the 4EBP phosphorylation level also exhibited a recovery after $8 \mathrm{~h}$ thaw, albeit still below control values (Fig. 5.5B). In terms of S6K, the protein level of S6K level dropped further while the phosphorylation level returned to control levels in muscle upon thawing, leading to a gross TORC1-dependent activation of S6K (Fig. 5.6). These data suggest that TORC1 activity recovered in muscle upon thawing. In addition, TORC1 function also recovered to some extent in heart with increased protein and phospho-4EBP levels as compared to frozen animals (Fig. 5.5), confirming the results from the CO-IP experiment (Fig. 5.8). However, neither freezing nor thawing produced appreciable changes in the protein and phosphorylation levels of S6K in heart (Fig. 5.6). Interestingly, heart Ser-2448 phosphorylation values for TOR do not restore after $8 \mathrm{~h}$ thaw, even with the phospho-S6K level staying at control levels. This may be due an inhibition of Akt signaling in heart upon thawing (Chapter 4), leading to a continuation of the low phospho-TOR (Ser-2448) level observed upon freezing. With 
regards to kidney, the phosphorylation level of both 4EBP and S6K recovered and further increased after $8 \mathrm{~h}$ thaw (Fig. 5.5B, 5.6B), suggesting activation of TORC1 activity. The results were consistent with the kidney CO-IP result where levels of the TORC1 complex recovered in thawed animals (Fig. 5.8). Interestingly, protein and phosphorylation levels of most TORC1 components did not recover or even dropped in kidney upon thawing as discussed above (Fig. 5.2-5.4). This may due to a slow recovery rate of kidney activity during thawing. Taken together, it appears that TORC1 assembly was resumed even with a low content of the individual components of the complex.

TSC2 and PRAS40 are two major inhibitory regulators of TORC1, both of which are directly regulated by the Akt pathway. Active Akt phosphorylates and inhibits TSC2 and PRAS40, thereby interrupting their interactions with TOR, ultimately promoting TORC1 assembly and function (Inoki et al., 2002; Haar et al., 2007). The data showed that the Akt-dependent phosphorylation level of TSC2 (Ser-939) dropped to around 50\% of the control level in muscle and heart after $24 \mathrm{~h}$ freezing exposure, but increased in liver (Fig. 5.7A). These results are consistent with the response of Akt signaling in wood frog to freezing stress where the pathway was activated in liver but inhibited in muscle and heart (Chapter 4). The decreased TSC2 phosphorylation level observed in muscle and heart matched the response of both TORC1 and its downstream targets responding to freezing stress. However, the phospho-PRAS40 level did not show appreciable changes in any of the four tissues during freezing (Fig. 5.7B), suggesting that inhibition of TORC1 in wood frogs during freezing is more dependent on TSC2 action rather than PRAS40. Upon thawing, the phosphorylation level of TSC2 dropped below control level in liver and remained low in muscle, while recovering in heart and increasing by almost 
2-fold in kidney (Fig. 5.7A). In terms of PRAS40, the Akt-dependent phosphorylation level increased in liver and muscle after $8 \mathrm{~h}$ thaw, but dropped to around $40 \%$ of the control value in heart (Fig. 5.7B). The liver and muscle results suggest that Aktdependent inhibition of PRAS40 might be responsible for the recovery of TORC1 assembly in these tissues, while heart and kidney were more TSC2-dependent. The data also indicate that, instead of universally phosphorylating downstream targets when activated, the Akt signaling pathway might be capable of regulating its downstream targets selectively.

Accordingly, the data in this chapter demonstrated the responses of TORC1 and its downstream targets, 4EBP and S6K, in liver, muscle, heart and kidney of wood frog under freeze-thaw conditions. Relative protein expression levels of key TORC1 complex components were assessed using immunoblotting. Since regulation of TORC1 assembly and activity is heavily dependent on phosphorylation events on complex components, the phosphorylation status of these components was also evaluated between the control and experimental animals. CO-IP experiments were used to investigate the status of the TORC1 complex. Since TORC1 play a critical role in initiating energy expensive mRNA translation (or protein synthesis), it is not a surprise that the function of the complex was suppressed in all tissues examined in wood frogs after prolonged whole body freezing. This suggests that inhibition of protein synthesis is an integral and important part of freeze-induced hypometabolism. Due to the kinase activity of TOR, TORC1-dependent regulation of its downstream targets is also via posttranslational phosphorylation. As expected, reduced levels of TORC1-dependent phospho-4EBP and phospho-S6K were observed in parallel with the inhibition of TORC1 during freezing exposure. In addition, 
the data show that TORC1 function was generally recovered after $8 \mathrm{~h}$ thawed. Tissue specific patterns in protein and phosphorylation levels of TORC1 components and its downstream targets were also observed in response to the freeze-thaw cycle. This might be due to the previously proposed tissue- and stress- specific responses of the Akt signaling pathway that is upstream of TORC1 (Chapter 4). This tissue-specific pattern of Akt signaling was further confirmed by our observations of the Akt-dependent phosphorylation levels of TSC2 and PRAS40. The present study demonstrated for the first time the regulation of TORC1 and its downstream targets in response to freeze-thaw cycles, suggesting that inhibition of TORC1 and the potential subsequent suppression of protein synthesis are integral parts of freeze tolerance by wood frogs. 
Fig. 5.1A

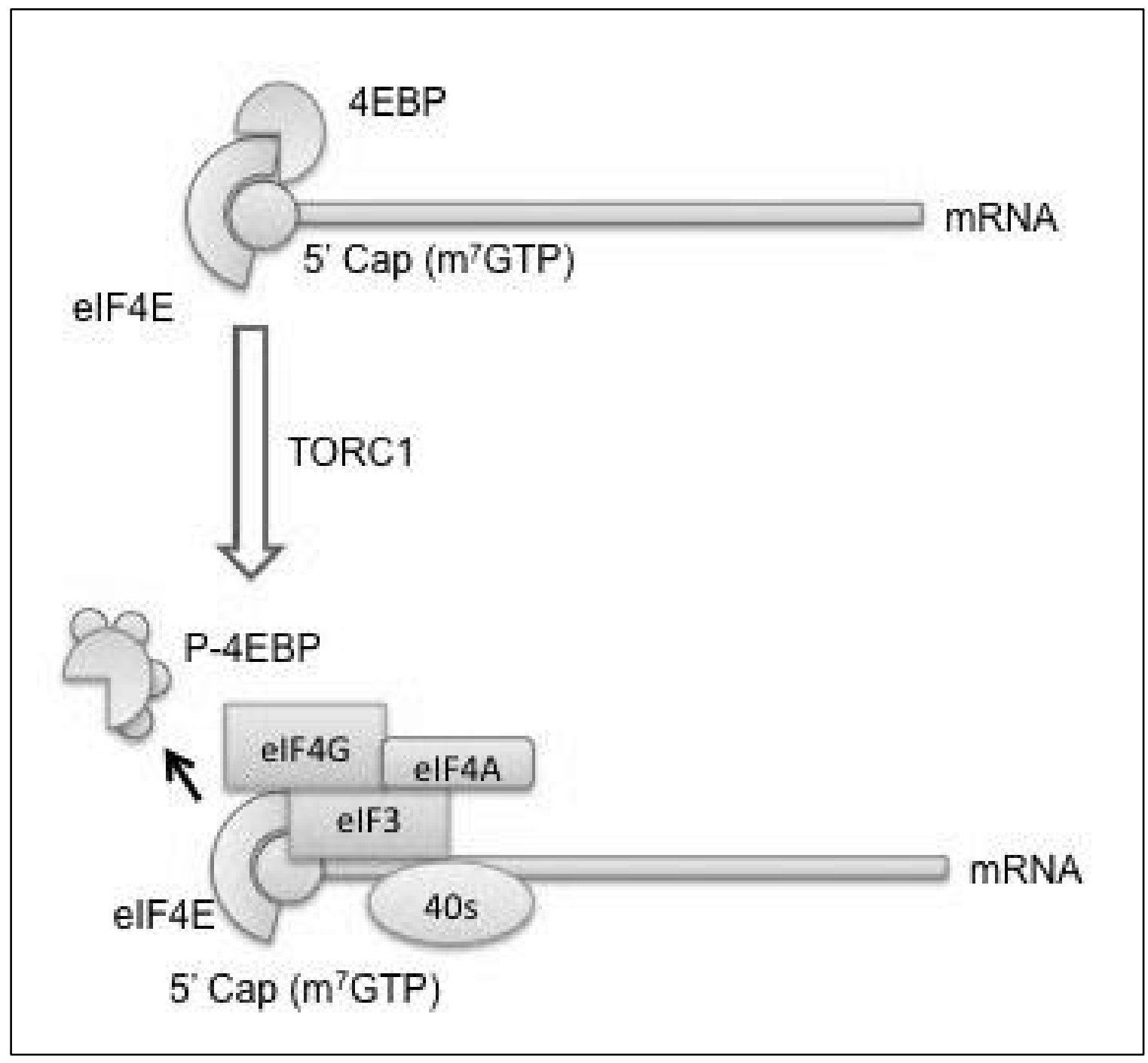


Fig. 5.1B

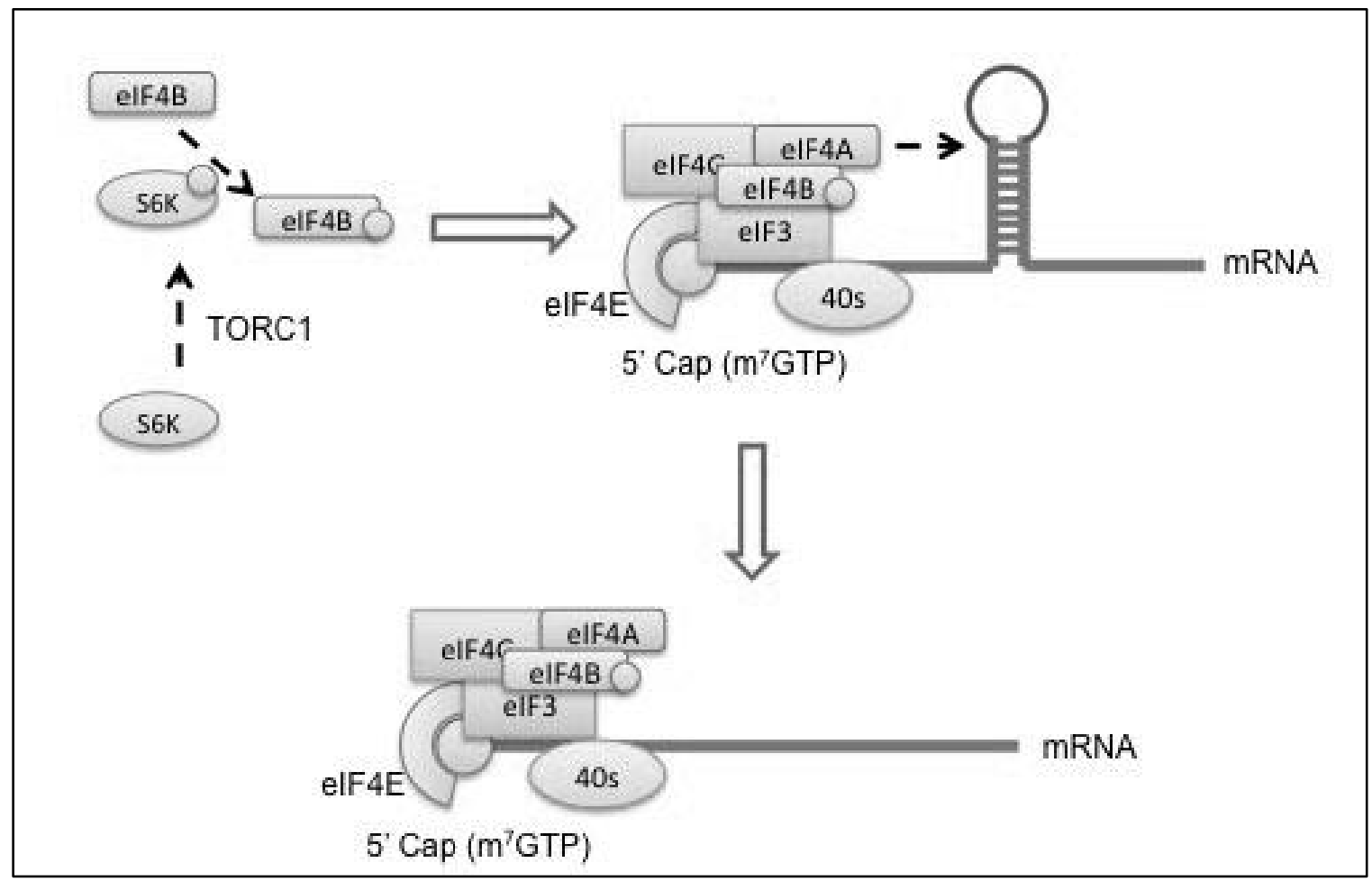

Fig. 5.1. TORC1-dependent mRNA translation initiation. (A) Inhibitory effect of TORC1 on 4EBP via phosphorylation. Phosphorylation on 4EBP by TORC1 promotes the dissociation of the protein from eIF4E, thereby stimulation the assembly of translation pre-initiation complex; (B) Stimulation of mRNA translation initiation by TORC1dependent S6K phosphorylation. TORC1-dependent phosphorylation on S6K stimulates its kinase function that phosphorylates eIF4B, which contributes to elimination of the inhibitory mRNA secondary structure. This eventually stimulates the formation translation pre-initiation complex. 
Fig. 5.2A
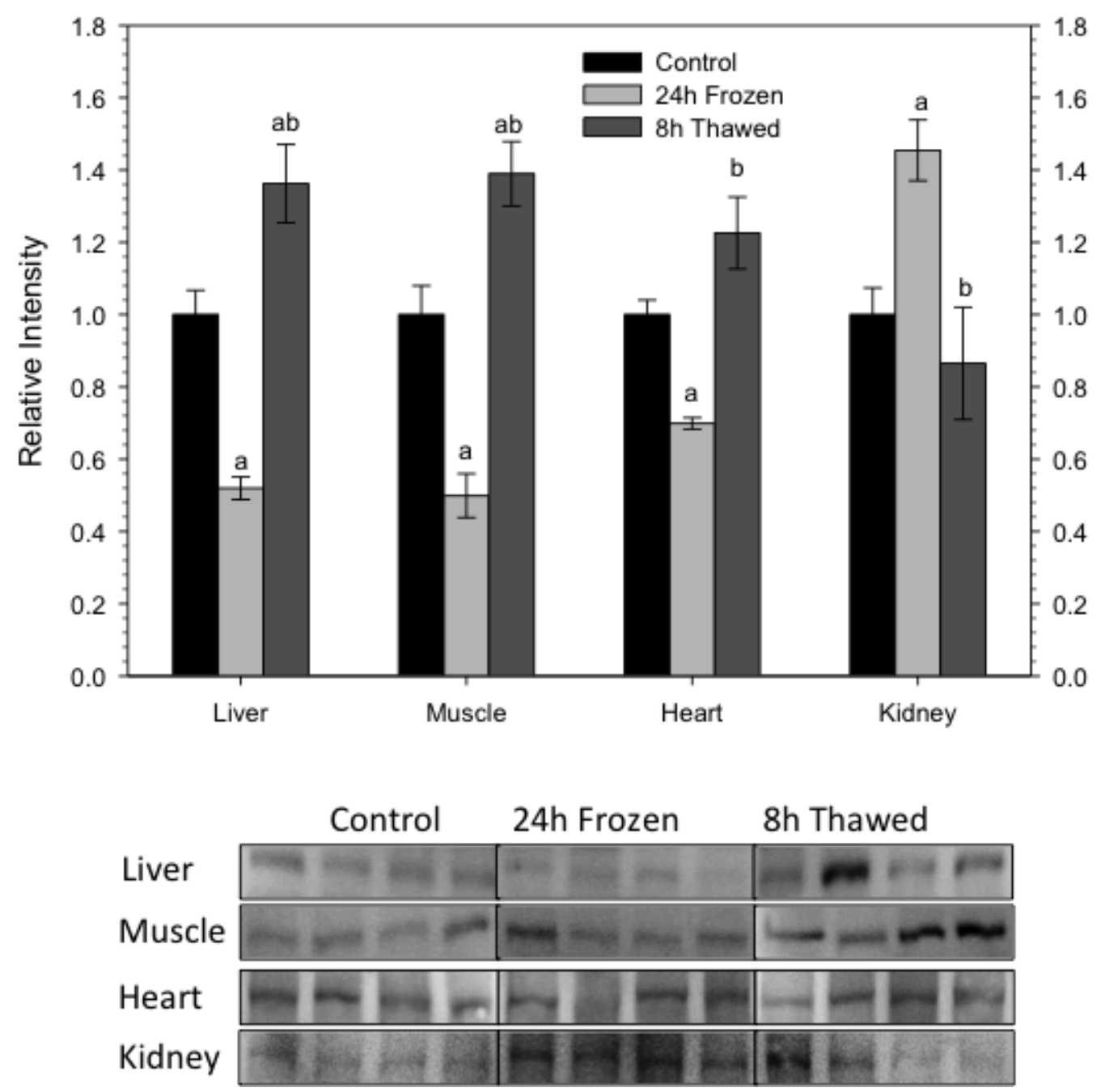
Fig. 5.2B
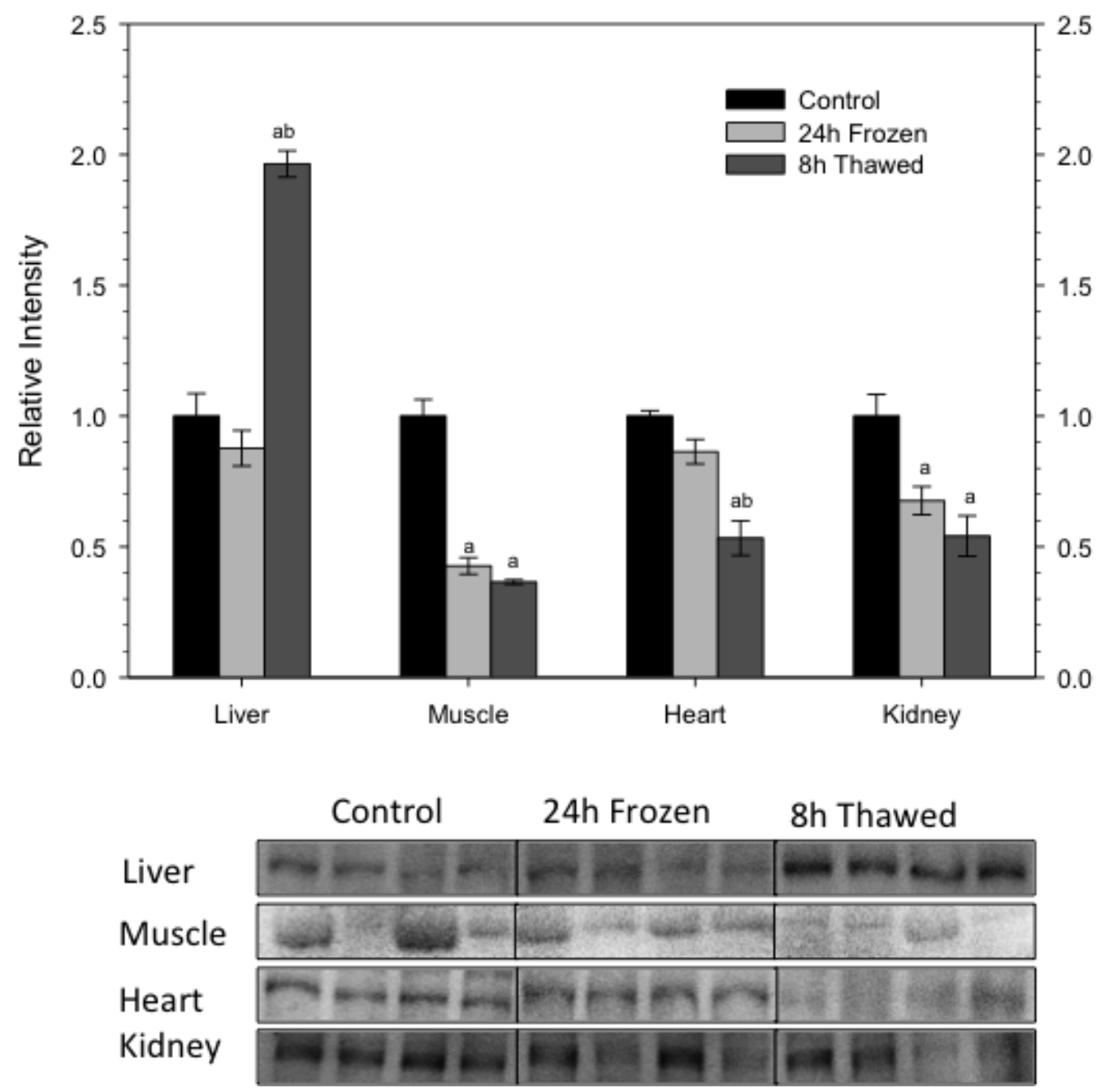

Fig. 5.2. Effects of $24 \mathrm{~h}$ freezing and $8 \mathrm{~h}$ thawing on the (A) total TOR protein, and (B) phosphorylation state of TOR at Ser-2448 level in liver, muscle, heart and kidney of wood frogs. Upper portion is the histogram showing standardized relative levels under control, $24 \mathrm{~h}$ frozen and $8 \mathrm{~h}$ thawed conditions; data are means \pm S.E.M., $\mathrm{n}=3-4$ independent trials on tissue from different animals. a - Significantly different from the corresponding control $(\mathrm{P}<0.05)$; $\mathrm{b}$ - significantly different from the $24 \mathrm{~h}$ frozen value. Lower portion of the figure shows representative immunoblot bands from each group. 
Fig. 5.3A
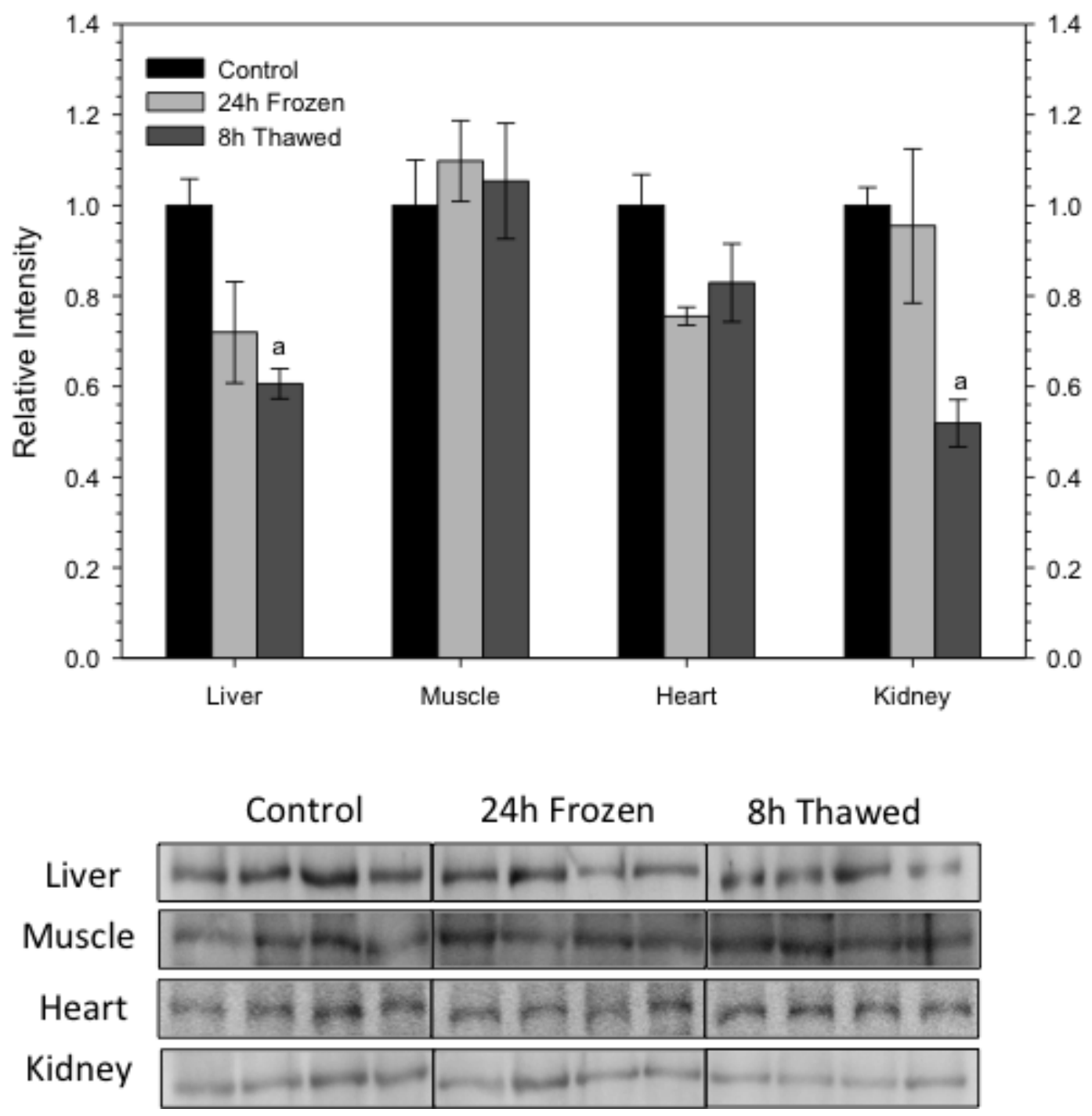
Fig. 5.3B
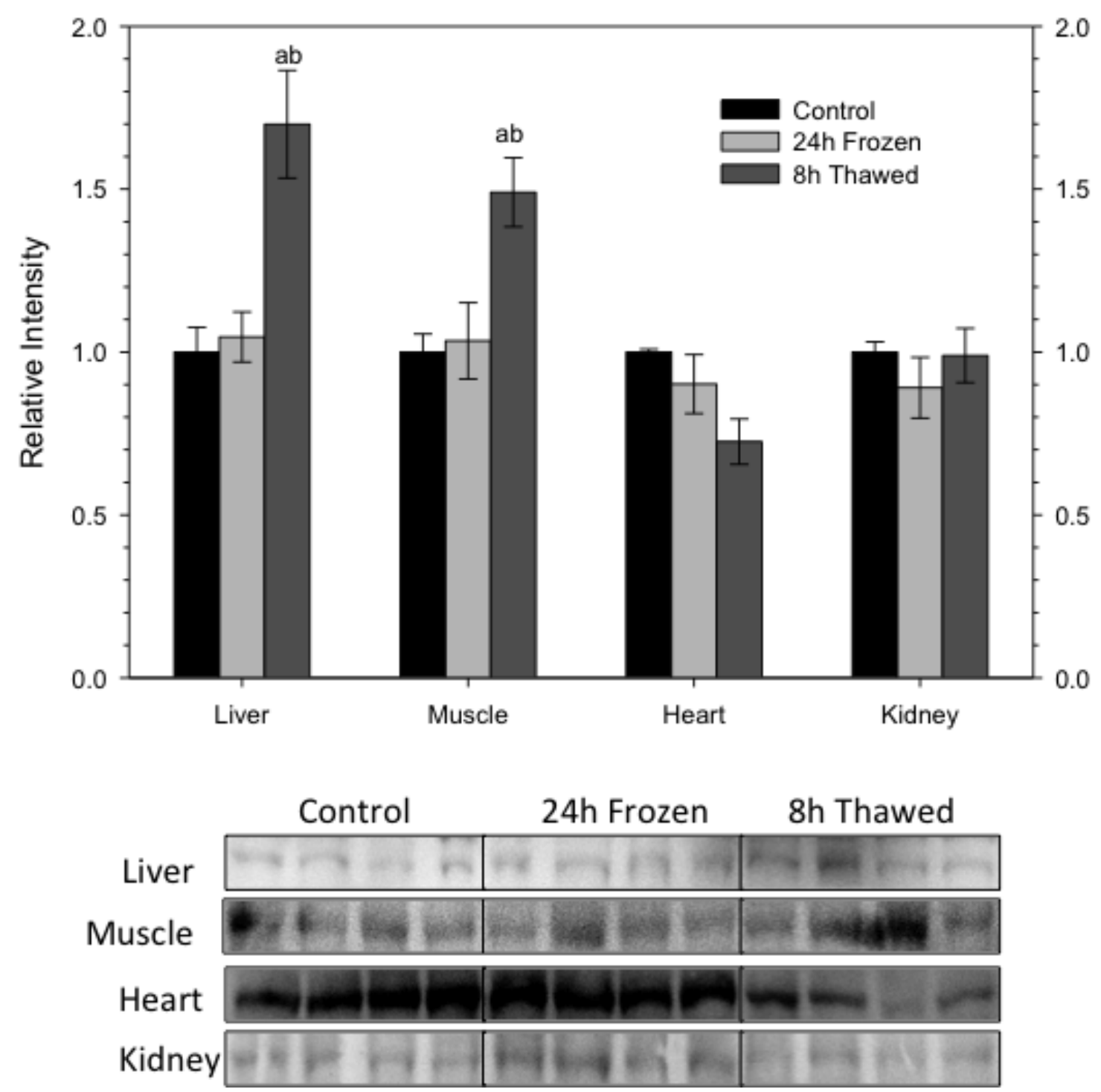

Fig. 5.3. Effects of $24 \mathrm{~h}$ freezing and $8 \mathrm{~h}$ thawing on the (A) total Raptor protein levels and (B) phosphorylation state (Ser-863) of Raptor in liver, muscle, heart and kidney of wood frogs. Other information as Fig. 1. 
Fig. 5.4
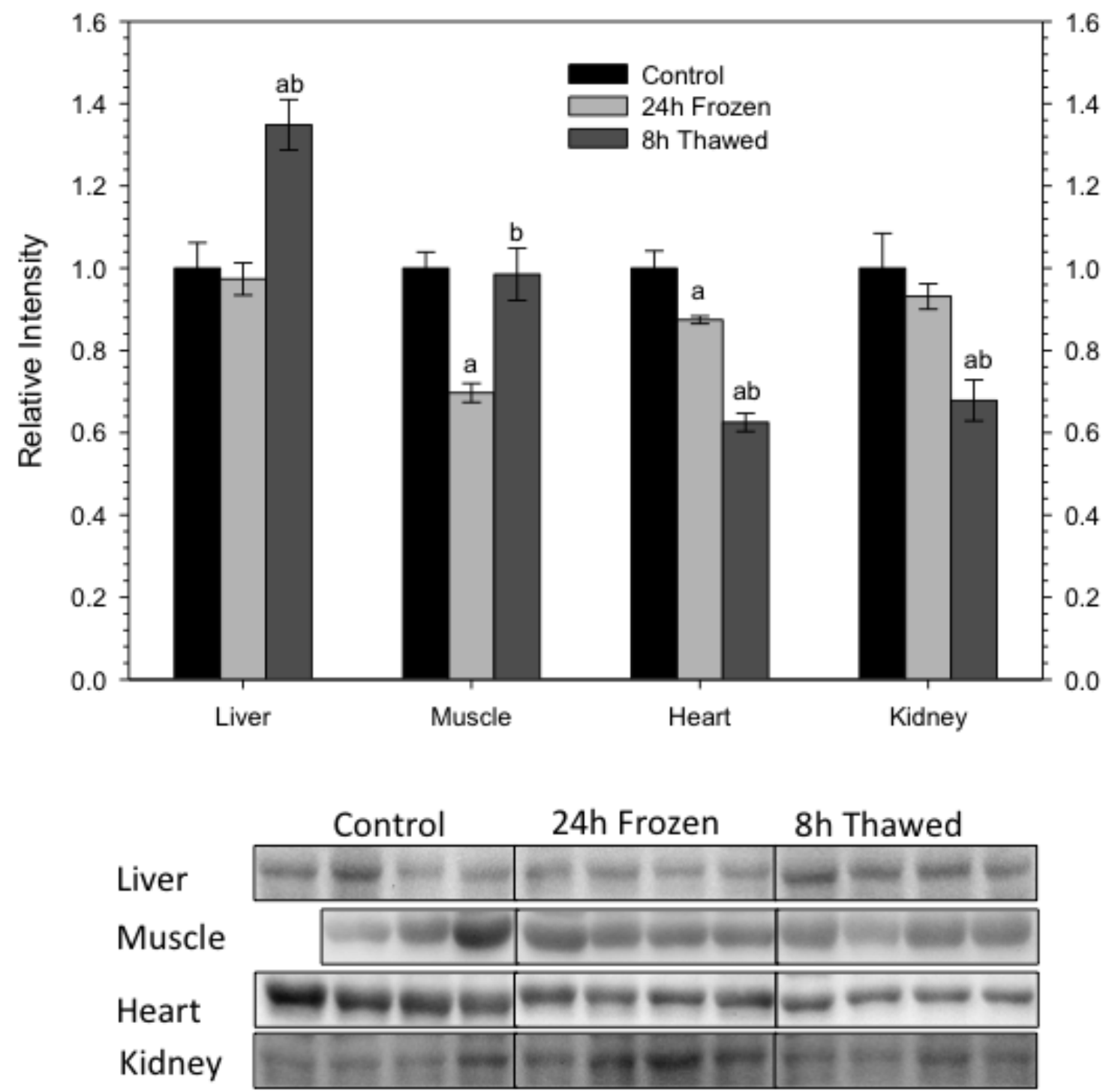

Fig. 5.4. Effects of $24 \mathrm{~h}$ freezing and $8 \mathrm{~h}$ thawing on the protein levels of G $\beta \mathrm{L}$ in liver, muscle, heart and kidney of wood frogs. Other information as Fig. 1. 
Fig. 5.5A
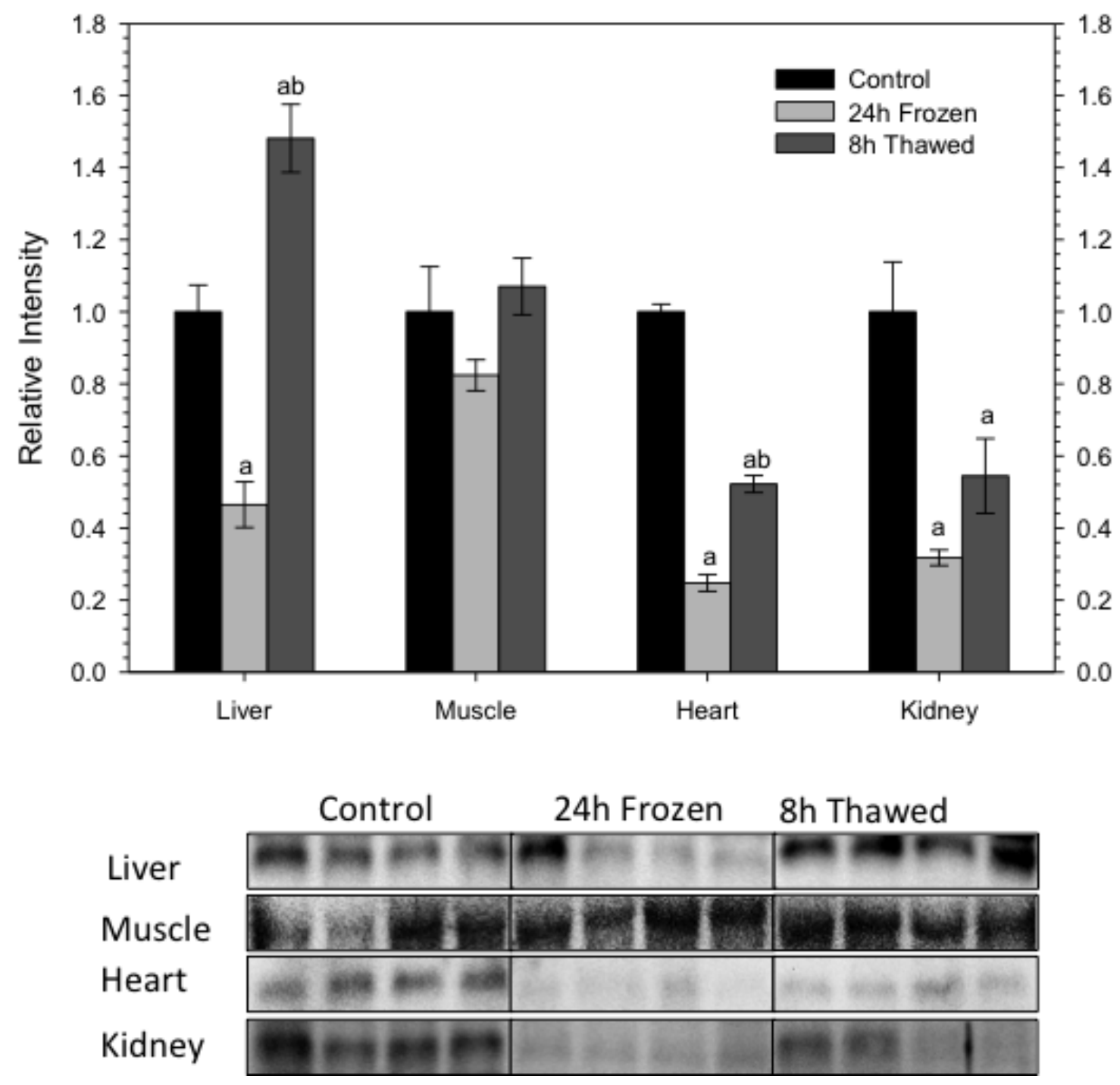
Fig. 5.5B
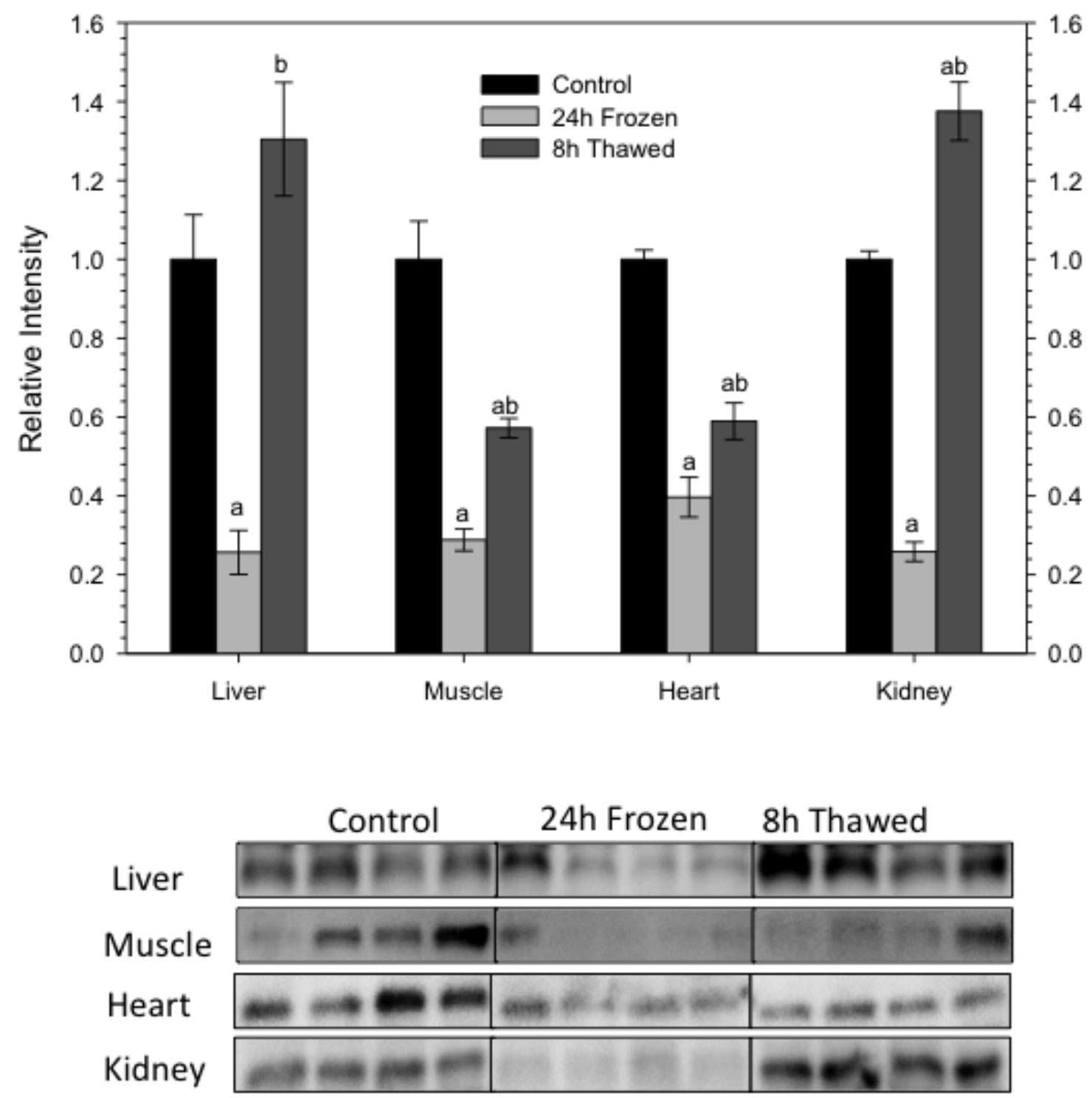

Fig. 5.5. Effects of $24 \mathrm{~h}$ freezing and $8 \mathrm{~h}$ thawing on (A) total $4 \mathrm{EBP}$ protein levels, and (B) relative phosphorylation (Thr-45) of 4EBP in liver, muscle, heart and kidney of wood frogs. Other information as Fig. 1. 
Fig. 5.6A
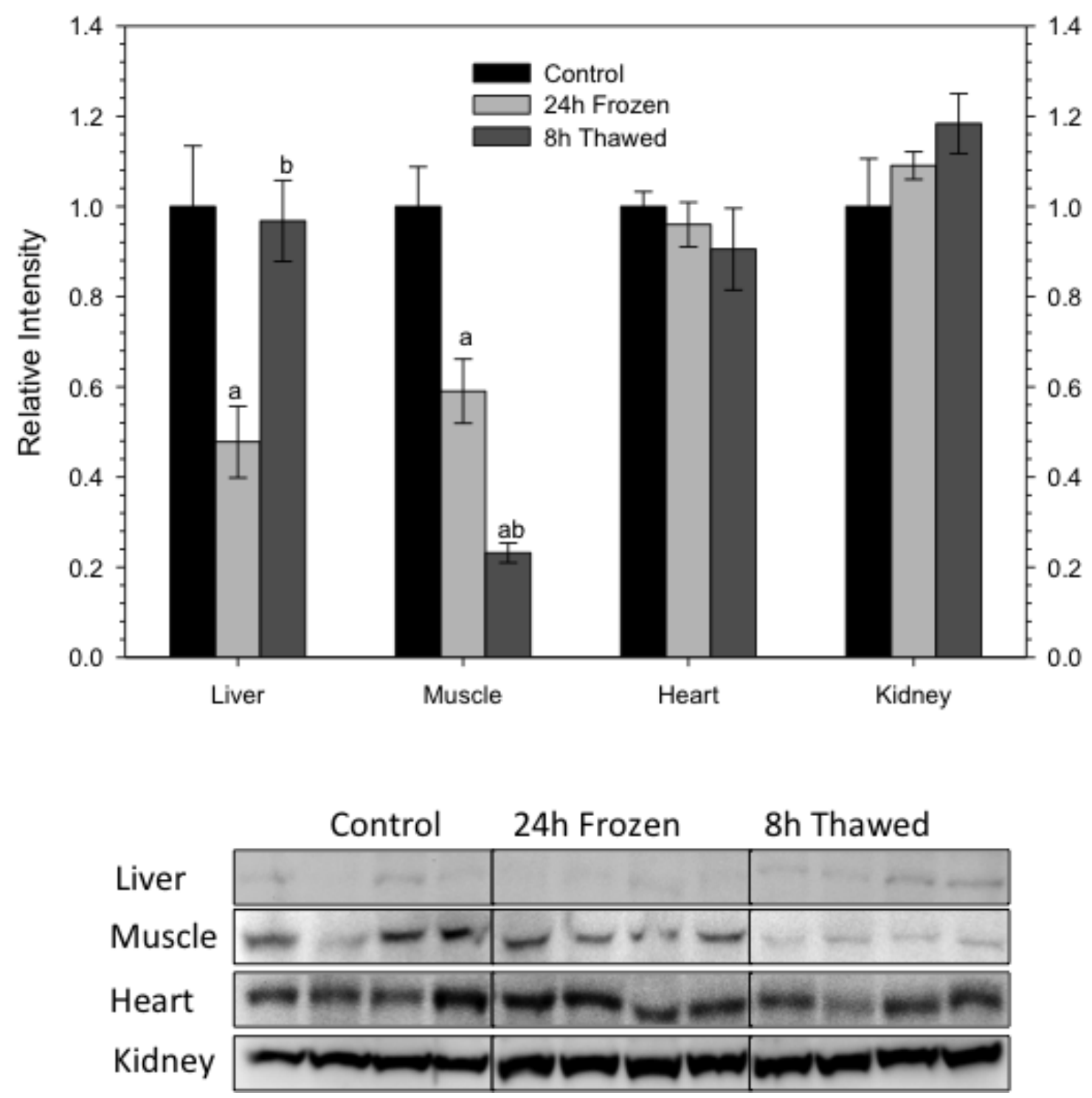
Fig. 5.6B
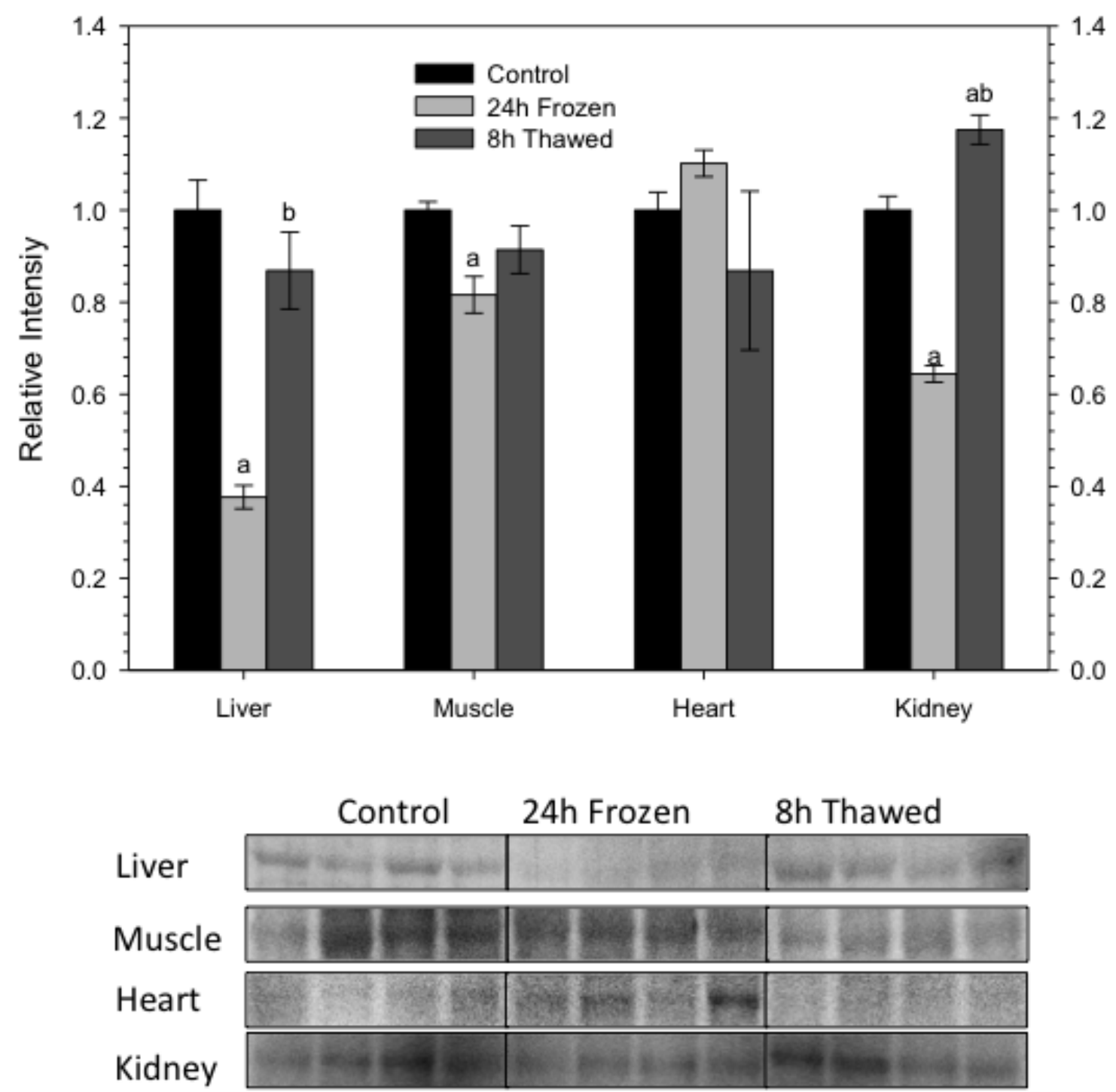

Fig. 5.6. Effects of $24 \mathrm{~h}$ freezing and $8 \mathrm{~h}$ thawing on (A) total S6K protein levels and (B) relative phosphorylation (Thr-389) of S6K in liver, muscle, heart and kidney of wood frogs. Other information as Fig. 1. 
Fig 5.7A
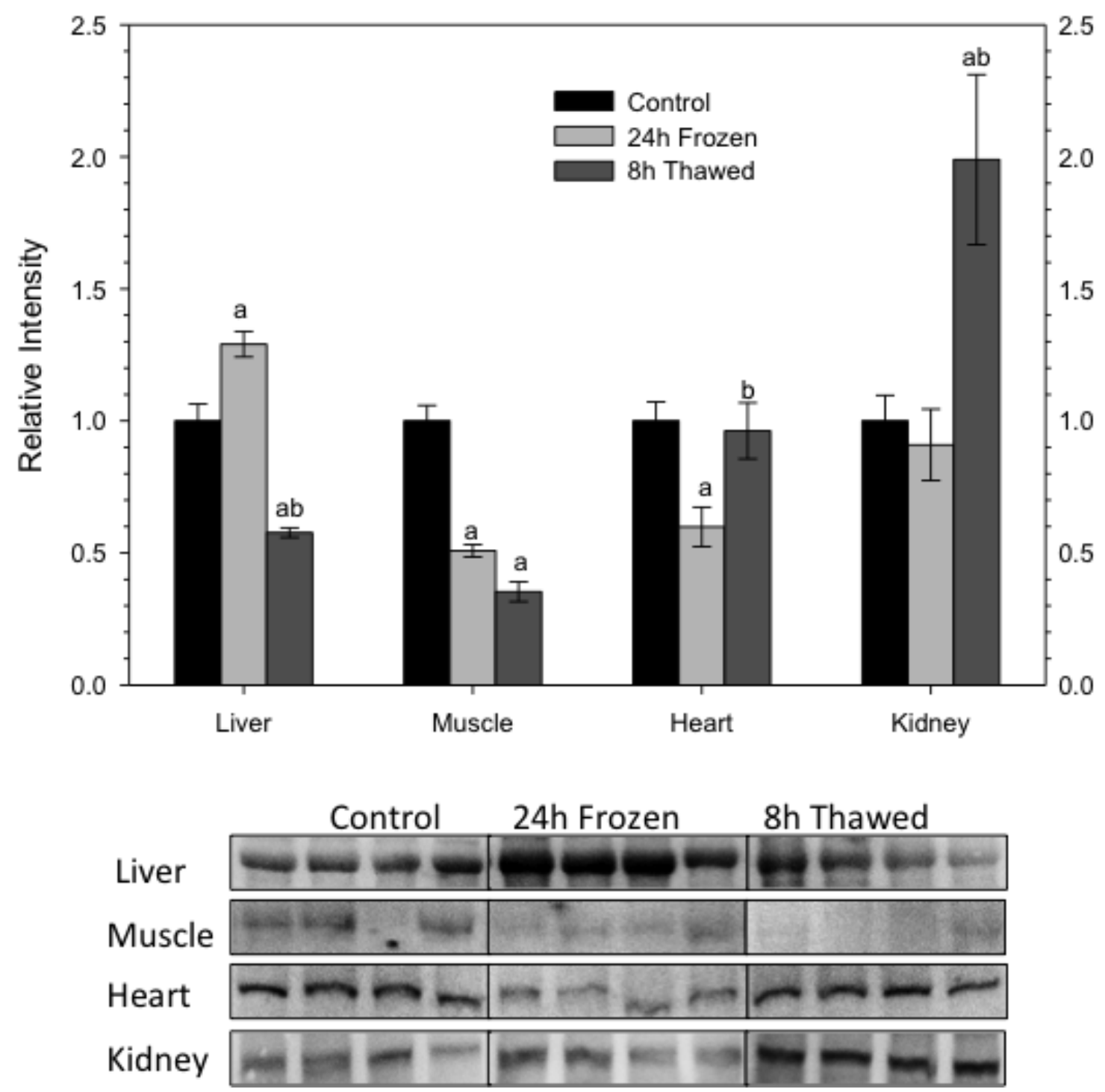
Fig. 5.7B
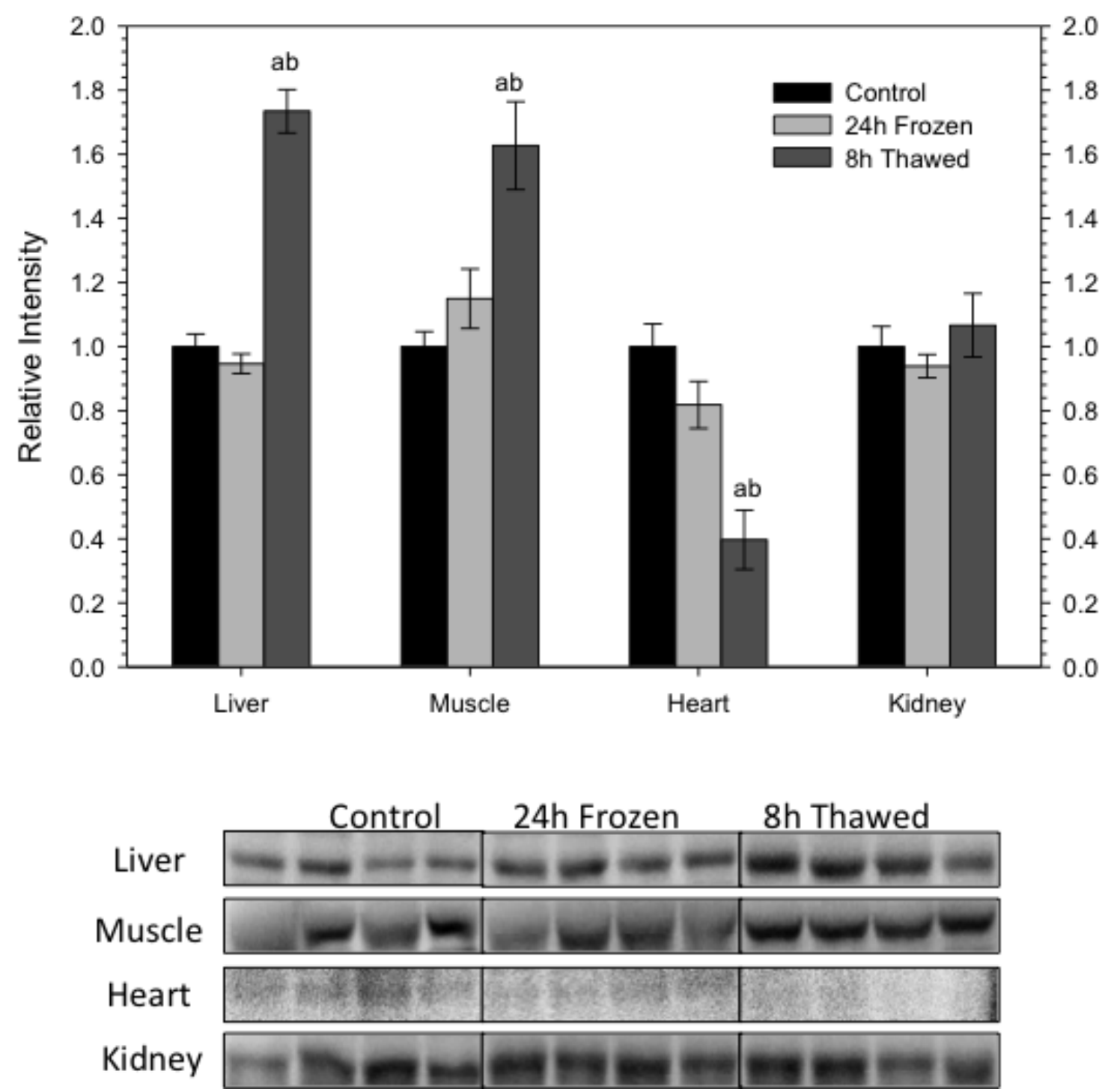

Fig. 5.7. Effects of $24 \mathrm{~h}$ freezing and $8 \mathrm{~h}$ thawing on the relative phosphorylation levels of (A) TSC2 (Ser-939), and (B) PRAS40 (Thr-246) in liver, muscle, heart and kidney of wood frogs. Other information as Fig. 1. 
Fig. 5.8

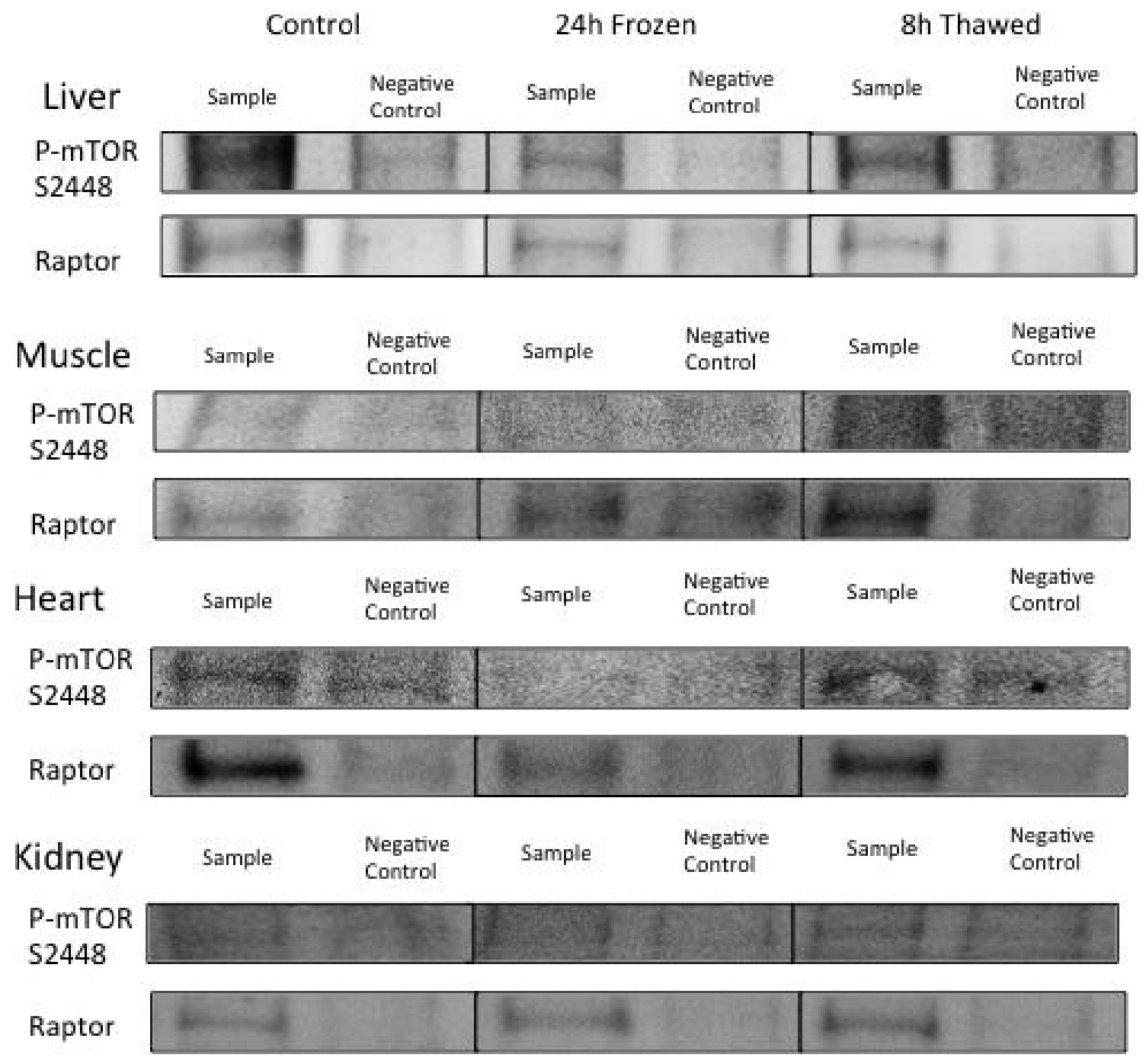

Fig. 5.8. Effects of $24 \mathrm{~h}$ freezing and $8 \mathrm{~h}$ thawing on P-TOR (Ser2448): Raptor interactions liver, muscle, heart and kidney as assessed by co-immunoprecipitation (CO-IP). For each tissue, the lower portion shows the Raptor immunoblot signal and the upper portion shows the P-TOR (Ser-2448) signal, both from the same sample precipitant. 


\section{Chapter 6 Nrf2-dependent antioxidant defense}




\section{Introduction}

Freezing in wood frogs begins in extracellular spaces and, as ice accumulates and excludes solutes from its crystal lattice, this sets up an imbalance between the osmolality of extracellular and intracellular fluids. As a result, there is a net outflow of intracellular water occurs into extracellular compartments, effectively placing a dehydration stress on cells (Storey and Storey, 2004a). Therefore, cell tolerance of dehydration stress is also a crucial part of freezing survival for wood frogs. Indeed, coordinated partial dehydration actually protects cells from potential physical damage that would arise if intracellular freezing occurred. However, too much dehydration of cells can also be damaging because compression stress on shrinking cells can cause an irreversible collapse of the membrane lipid bilayer structure into a gel state. Therefore, in order to constrain cellular water loss while still facilitating the partial dehydration that is beneficial to freezing survival, glucose is accumulated as a cryoprotectant by wood frog tissues. Glucose is produced in large quantities by liver, exported using the GLUT 2 transporter in liver cell membranes, distributed by the blood, and taken up by organs. The high intracellular osmolality created by glucose then defends cells against excessive and irreversibly damaging water loss during freezing. During freezing exposure, wood frogs are able to maintain extremely high glucose levels $(100 \sim 300 \mathrm{mM})$, in comparison with only $\sim 5 \mathrm{mM}$ in control frogs (Storey and Storey, 2004a).

In addition, as introduced in Chapter 1, prolonged freezing exposure leads to anoxic conditions in wood frogs, due to the interruption of gas exchange caused by plasma solidification. By contrast, during thawing, frog organs can experience oxidative stress due to a rapid production of ROS when oxygen is re-introduced to tissues when 
breathing and circulation resume. Previous studies demonstrated that antioxidant defense mechanisms were activated in wood frogs facing freeze-thaw cycles (Joanisse and Storey, 1996). In recent years, studies have reported stress responsive regulation of antioxidant defense-related cell signaling pathways and gene expression in several stress tolerant animal species (Krivoruchko and Storey, 2010b; Malik and Storey, 2011; Allan and Storey, 2012). Furthermore, it has been established that hyperglycemia can induce oxidative stress through multiple metabolic pathways in the context of diabetes (King and Loeken, 2004). A study by Joanisse and Storey (1996) assessed responses of several key antioxidant enzymes such as catalase, superoxide dismutase (SOD) and glutathione S-transferase (GST) in wood frogs experiencing freeze-thaw cycles, suggesting a similar antioxidant defense network occurring in wood frogs as in other model systems (Harris, 1992). With the recent development of multiplex Luminex technology, multiple antioxidant enzymes can be assessed at the same time. The present chapter takes advantage of this technology and furthers the study by Joanisse and Storey (1996) by examining the protein expression levels of multiple antioxidant enzymes including catalase, PRX2 (Peroxiredoxin 2), SOD1 (Cu/Zn SOD, copper/zinc dependent superoxide dismutase), SOD2 (MnSOD, manganese dependent superoxide dismutase) and TRX1 (Thioredoxin 1).

In addition, the Nrf2 transcription factor plays crucial roles in the ARE-dependent antioxidant defense machinery. Nrf2 binds to the ARE sequence (TGAG/CNNNGC) that is present in the promoter region of many genes involved in antioxidant defense and detoxification of reactive electrophilic compounds (e.g. GSTs, SODs, heme oxygenase-1, etc.), as well as those that are involved in glutathione (GSH) synthesis, e.g. GR 
(glutathione reductase) (Alam et al., 1999; Chanas et al., 2002; Park and Rho, 2002; Harvey et al., 2008). When inactive, Nrf2 is sequestered outside of the nucleus through its interaction with the inhibitor protein Keap1. Such interaction also creates the necessary structural features required for ubiquitin-dependent Nrf2 degradation (Katoh et al., 2005; Zhang, 2006; Kaspar et al., 2009). When cells experience oxidative stress, Keap1 undergoes a conformational change that leads to the release of Nrf2. In addition, Nrf2 is also subject to PKC (protein kinase C)-dependent phosphorylation events that contribute to inhibition of the Nrf2-Keap1 interaction. After dissociating from Keap1, Nrf2 then localizes into the nucleus where it promotes antioxidant defense-related gene expression (Huang et al., 2002; Dinkova-Kostova et al., 2002; Kaspar et al., 2009). Furthermore, GSK3 $\beta$ (glycogen synthase kinase $3 \beta$ ) also plays a role in regulating Nrf2 in an Akt/PKB-dependent fashion. GSK3 $\beta$-dependent phosphorylation of Nrf2 sequesters Nrf2 into the cytoplasm and facilitates degradation of the protein (Salazar et al., 2006; Jain and Jaisweal, 2007; Rada et al., 2011). Upon activation, Akt/PKB phosphorylates and inhibits GSK3 $\beta$ which ultimately relieves the inhibitory effect of GSK3 3 on Nrf2 (Pearl et al., 2002).

The present chapter furthers the previous findings about the role of antioxidant defenses in freeze tolerance of wood frogs (Joanisse and Storey, 1996) by examining protein expression levels of key antioxidant enzymes using a Multiplex assay. In addition, the study examines Nrf2-dependent antioxidant responses in wood frog tissues by measuring the relative protein expression level of $\mathrm{Nrf} 2$, the phosphorylation state of GSK3 $\beta$, transcript levels of gstal and Nrf2-DNA binding activity in liver and muscle of wood frogs. Since glucose as the cryoprotectant plays critical roles wood frog freeze 
survival, the effects of glucose on Nrf2-DNA binding activity in vitro are also explored as well as the antioxidant capacity in glucose-loaded frogs. The study demonstrates that Nrf-2 dependent antioxidant defense is an integral part of freeze tolerance by wood frogs. Furthermore, I propose that glucose cryoprotectant may also facilitate antioxidant defence in wood frogs by regulating Nrf2-DNA binding activity.

\section{Results}

Plasma glucose levels in wood frogs $2 \mathrm{~h}$ after saline and glucose injection

To investigate the effects of high glucose on antioxidant defense mechanisms in wood frogs, intraperitoneal injection of glucose (a volume of $2 \mathrm{M}$ glucose in saline equivalent to $8 \%$ of body mass) into $5^{\circ} \mathrm{C}$-acclimated frogs was used to elevate sugar levels into the range that is induced naturally by freezing. Table 6.1A shows that glucose injection into frogs elevated plasma glucose levels to $191.0 \pm 8.74 \mathrm{mM}$ by $2 \mathrm{~h}$ postinjection with $2 \mathrm{M}$ glucose solution; this is comparable to the plasma glucose level (200 300 mM) present in frozen frogs. However, after $6 \mathrm{~h}$ the glucose injected frogs showed a lower plasma glucose value of $\sim 178 \mathrm{mM}$ which suggests that glucose clearance was underway. Therefore, $2 \mathrm{~h}$ glucose-loaded frogs were used in the further analyses described below.

In addition, table $6.1 \mathrm{~B}$ shows that the glucose levels measured using the glucose assay kit matched with the calculated theoretical value $(0.69 \mathrm{mM}$ glucose standard solution mixed with equal volume of 50x diluted blood plasma from the $6 \mathrm{~h}$ glucose 
loaded frog), meaning that the in vivo environment of the wood frog didn't interfere with the assay.

Effect of freeze-thaw and glucose on protein levels of antioxidant defense-related enzymes in liver and muscle of wood frogs

Relative protein levels of catalase, PRX2, SOD1, SOD2 and TRX1 were assessed in liver and muscle of control, $24 \mathrm{~h}$ frozen, $8 \mathrm{~h}$ thawed, $2 \mathrm{~h}$ saline-loaded and $2 \mathrm{~h}$ glucoseloaded wood frogs, using a 5-plex Luminex panel. Freeze-thaw had no effect on the relative protein levels of catalase, SOD1 and SOD2 in liver (Fig. 6.1A). However, the protein level of liver PRX2 dropped significantly to $61 \pm 1.4 \%$ of the control value after 24 $\mathrm{h}$ freezing exposure and remained low at $51 \pm 5.8 \%$ of the control after $8 \mathrm{~h}$ thawed. TRX1 protein levels were also decreased significantly in liver of $8 \mathrm{~h}$ thawed frogs, falling to $67.3 \pm 3 \%$ of the control values (Fig. 6.1A). In terms of the glucose-injected frogs, when compared with the saline-injected group, protein levels of catalase, PRX2 and SOD2 decreased significantly to $47.2 \pm 3.9 \%, 63.5 \pm 6.8 \%$ and $83.5 \pm 4.2 \%$ of the control values in liver, respectively, whereas SOD1 and TRX1 were unchanged (Fig. 6.1B).

In terms of muscle, most targets tested did not show significant changes during freeze-thaw (Fig. 6.2A). Only PRX2 exhibited increased protein levels in both frozen and thawed frogs by $1.57 \pm 0.09$ - and $1.55 \pm 0.15$-fold, respectively. Similar to the response to freeze-thaw, only PRX2 showed a small but statistically significant change in response to glucose injection as compared to saline-loaded frogs; protein levels decreased to $87.4 \pm 3.1 \%$ of the value for $2 \mathrm{~h}$ saline loaded frogs (Fig. 6.2B). 
Glucose effect on antioxidant capacities in liver and muscle of wood frogs

Relative antioxidant capacities were examined in liver and muscle of $2 \mathrm{~h}$ salineloaded versus $2 \mathrm{~h}$ glucose-loaded wood frogs using a commercially available assay kit. The assay examines the inhibitory effect of tissue homogenates on the metmyoglobindependent oxidation of ABTS (2,2'-Azino-di-[3-ethylbenzthiazoline sulphonate]). The results are presented as the concentration of the standard antioxidant Trolox (associated with the kit) equivalent to the antioxidant capacity of the sample. Fig 6.3A shows that antioxidant capacity increased significantly with a fold change of $2.8 \pm 0.1$ in skeletal muscle of $2 \mathrm{~h}$ glucose-loaded frogs as compared with the saline injected group. Liver, on the other hand, did not show appreciable changes in antioxidant capacity after glucose injection.

Effect of freeze-thaw and glucose on protein levels of Nrf2 in liver and muscle of wood frog

Relative protein levels of Nrf2 were assessed in liver and muscle of control, $24 \mathrm{~h}$ frozen, $8 \mathrm{~h}$ thawed, $2 \mathrm{~h}$ saline-loaded and $2 \mathrm{~h}$ glucose-loaded wood frogs using immunoblotting. Fig. 6.4A shows that the relative protein levels of Nrf2 increased significantly in liver and muscle of thawed frogs as compared to frozen frogs; however, no appreciable changes were observed between control and frozen frogs. Glucose-loading resulted in a 1.4 \pm 0.03 -fold increase in Nrf2 protein levels in liver as compared with saline injected frogs but no appreciable change occurred in muscle (Fig. 6.4B). 
Effect of freeze-thaw cycle and glucose loading on protein levels and phosphorylation state of GSK3 $\beta$ in liver and muscle of wood frogs

Relative protein and phosphorylation (Ser-9) levels of GSK3 $\beta$ were assessed in liver and muscle of control, $24 \mathrm{~h}$ frozen, $8 \mathrm{~h}$ thawed, $2 \mathrm{~h}$ saline-loaded and $2 \mathrm{~h}$ glucoseloaded wood frogs using immunoblotting. Fig. 6.5A shows that the relative protein levels of GSK $3 \beta$ decreased in the liver of $24 \mathrm{~h}$ frozen frogs to $70.1 \pm 4.4 \%$ of control values and remained low after $8 \mathrm{~h}$ thawed $(67.7 \pm 1.6 \%$ compared with controls). In muscle, however, no appreciable changes in GSK3 $\beta$ protein levels occurred over freeze-thaw. As shown in Fig. 6.5B, relative GSK3 $\beta$ protein levels strongly increased by $4.4 \pm 0.71$-fold in liver of $2 \mathrm{~h}$ glucose-loaded frogs as compared saline-injected frogs. However, muscle GSK3 $\beta$ protein levels were unaffected by glucose loading.

As shown in Fig. 6.6A, relative phosphorylation levels of GSK3 $\beta$ (Ser-9) increased by $1.78 \pm 0.09$-fold in the liver of $24 \mathrm{~h}$ frozen frogs but returned to control values after $8 \mathrm{~h}$ thawed. In muscle, however, the relative amount of Ser9 phosphorylated GSK3 $\beta$ decreased to $53.1 \pm 3.7 \%$ of the control value during freezing and remained low after thawing ( $42.6 \pm 3.2 \%$ of the control value). Compared with saline injected frogs, the phosphorylation level of GSK3 $\beta$ deceased to $67.8 \pm 3.3 \%$ in muscle after glucose injection but high glucose failed to trigger a significant change in liver (Fig. 6.6B).

Effect of freeze-thaw and glucose loading on DNA binding activities of Nrf2 in wood frog tissues 
An EMSA experiment was utilized to verify the DNA probes containing ARE sequences using liver nuclear extracts from control frogs. The results of the gel shift analysis show that proteins in frog liver tissue nuclear extracts were able to interact with the ARE probe. As shown in Fig. 6.7A, two bands were detected in the sample lane (third lane) but were not seen in the no probe and no protein controls (first and second lanes, respectively). An antibody supershift reaction was also run to confirm that the signal(s) in the sample lane was actually caused by Nrf2 protein. For the super shift reaction, $1 \mu 1$ of primary antibody specific for $\mathrm{Nrf} 2$ was added after incubation of the nuclear extract with the ARE probe and allowed to further incubate at room temperature for $2 \mathrm{~h}$ before gel electrophoresis. The figure shows that, when compared with the sample lane, the signals of probe shift were absent at both positions as compared with the supershift lane 4 , suggesting that the shift signals in lane 3 were Nrf2 specific. EMSA results also show Nrf2 shift presented as two bands in wood frog, agreeing with data from mammalian models (Zhao et al., 2007).

DNA binding activities of Nrf2 were assessed in nuclear extracts of liver and muscle of control, $24 \mathrm{~h}$ frozen and $8 \mathrm{~h}$ thawed wood frogs using a transcription factor DNA binding assay. Fig. 6.7B shows that DNA binding activity increased by $1.56 \pm$ 0.04 -fold in liver of $24 \mathrm{~h}$ frozen wood frogs but then decreased strongly to $50.5 \pm 4.7 \%$ of the control value after $8 \mathrm{~h}$ thawed. In muscle, Nrf2 DNA binding activity also increased significantly during freezing with a fold increase of $1.78 \pm 0.2$ over controls but decreased somewhat after thawing.

The effect of glucose on the DNA binding activity of Nrf2 was assessed in vitro by the same method in reactions containing 200 or $500 \mathrm{mM}$ glucose added to nuclear 
extracts from liver and muscle of control frogs. As shown in Fig. 6.7C, the presence of $200 \mathrm{mM}$ glucose resulted in increased DNA binding activity by Nrf2 in liver extracts, as compared with no glucose controls; binding significantly increased by $2.02 \pm 0.11$ fold. Binding remained high after thawing at $1.65 \pm 0.2$ fold over controls. In muscle, Nrf2 DNA binding activity also increased significantly by $2.59 \pm 0.4$-fold in the presence of 200 $\mathrm{mM}$ glucose as compared to no glucose controls. However, $500 \mathrm{mM}$ glucose failed to change the DNA binding activity of Nrf2 in muscle (Fig. 6.7C).

Effect of freeze-thaw cycle as well as glucose on the transcript levels of gstal in liver and muscle of wood frogs

Transcript levels of gstal, a downstream gene under Nrf2 control, were assessed in liver and muscle of control, $24 \mathrm{~h}$ frozen, $8 \mathrm{~h}$ thawed, $2 \mathrm{~h}$ saline-loaded and $2 \mathrm{~h}$ glucoseloaded wood frogs, using RT-PCR. Fig. 6.8A shows that the transcript levels of gstal were elevated by $1.3 \pm 0.03$-fold in liver after $24 \mathrm{~h}$ freezing exposure but returned to control values after an $8 \mathrm{~h}$ thaw. However, no changes were observed in muscle gstal transcript levels after freeze-thaw. By contrast with freezing, glucose injection suppressed the transcript levels of gstal which fell to $61.1 \pm 2.6 \%$ of saline injected values in liver, whereas no appreciable changes were observed in muscle (Fig. 6.8B).

\section{Discussion}

One of the challenges freeze tolerant wood frogs must confront when experiencing whole body freezing is the interruption of oxygen exchange through blood 
flow, creating an ischemic condition. With the overall metabolic rate being suppressed throughout the freezing exposure, energy expenditure needs to be reduced and reprioritized to maintain the basal metabolism for survival as well as to activate stress responsive pathways and the expression of selected genes that aid cell preservation. Switching from aerobic to anaerobic metabolism in the frozen state and restoring metabolism to normal after thawing also effectively creates ischemia-reperfusion conditions such as has have been well-studied in other systems as a major circumstance for ROS production and oxidative damage (Campos et al., 1993; Downey, 1990; Fuller et al., 1988; Ruuge et al., 1991; Traystman et al., 1991). Although pathways and/or adaptations involved may vary, antioxidant mechanisms have been demonstrated to play critical roles in freeze survival by various freeze tolerant species. For example, metalbinding protein-dependent antioxidant defense mechanisms play a key role in the freeze tolerant intertidal gastropod Littorina littorea, whereas freeze tolerant hatching painted turtles (Chrysemys picta marginata) exhibited up-regulated ferritin heavy and light chains during stress exposure (English and Storey, 2003; Storey, 2006). These proteins also showed signs of activation in wood frogs (Storey, 2004). In addition, accumulation of glucose in wood frogs during freezing may lead to glucose-related ROS production, given that diabetic conditions are also considered to be a major source of oxidative tissue damage (Kristal and Yu, 1992; King and Leoken, 2004). This adds another layer of potential oxidative pressure to wood frogs when facing whole body freezing. Therefore, freeze tolerance by wood frogs must include antioxidant defense adaptations. In fact, previous studies reported that several antioxidant enzymes were more active in the liver of wood frog compared to the non-freeze tolerant leopard frogs; wood frogs also showed 
higher GSH levels than leopard frogs (Joanisse and Storey, 1996). These data suggest an overall higher antioxidant capacity of the freeze tolerant wood frogs.

Starting from investigating the protein expression levels of key antioxidant enzymes in response to the freeze-thaw cycle using a multiplex assay, the studies reported in the present chapter focus on the antioxidant response in muscle and liver of wood frogs when facing such stress conditions. The transcription factor Nrf2 was chosen as the core of the study because it controls the expression of a variety of genes encoding multiple key antioxidant factors. Furthermore, high glucose content in wood frogs when experiencing ischemic conditions during freezing exposure may impose a possibility of glucose being involved in antioxidant defense mechanisms. Therefore, the present study investigated the effects of glucose-loading on the antioxidant response (with a focus on Nrf2-related mechanisms). As shown in Table 6.1, plasma glucose levels reached around $200 \mathrm{mM}$ by $2 \mathrm{~h}$ post-injection with a bolus of $2 \mathrm{M}$ glucose (Table 6.1B).

The protein expression levels of five major antioxidant enzymes (catalase, PRX2, SOD1, SOD2 and TRX1) were assessed in liver and muscle of frozen, thawed and saline/glucose injected frogs (Figs. 6.1, 6.2). SOD is a very important antioxidant enzyme, providing a first-line of antioxidant defense to deal with superoxide generated from a variety of biological reactions. The function of SOD is to convert superoxide radical to the less reactive hydrogen peroxide (Oberley and Buettner, 1979). SOD1 and SOD2 are the cytoplasmic and mitochondrial isoforms, respectively (Muller $\boldsymbol{e t}$ al., 2007). Catalase and PRX2 complete the work started by SOD by converting hydrogen peroxide into water and oxygen (Kirkman and Gaetani, 1984; Zhu et al., 2012). PRX2 is also able to reduce alkyl hydroperoxides to alcohols (Low et al., 2007). During the 
detoxification process, disulphide bridges form between subunits of PRX2. TRX1 is responsible for reducing these disulphides back to free cysteine residues to allow renewed PRX2 function (Low et al., 2007). Although with some exceptions, the multiplex results suggest that freeze-thaw cycle generally did not affect the protein levels of most enzymes tested in both liver and muscle (Figs. 6.1A, 6.2A). This agrees with the energy preservation strategy of minimizing protein synthesis during the frozen hypometabolic state. Interestingly, PRX2 exhibited opposite results in liver and muscle where its protein levels decreased in liver (Fig. 6.1A) but increased in muscle (Fig. 6.2A) in response to freeze-thaw. Consistent with the decrease of PRX level upon thawing, TRX1 level also dropped in liver (Fig. 6.1A).

In order to explore the effect of high glucose on the level of these antioxidant enzymes, the multiplex analysis was also carried out on tissues from $2 \mathrm{~h}$ glucose-loaded frogs. The protein levels of catalase, PRX2 and SOD2 were lowered in the presence of high glucose content in liver (Fig. 6.1B). However, the protein levels of most enzymes tested in muscle were not affected by glucose injection, with only a slight drop of PRX2 levels (Fig. 6.2B). Tissue specific patterns emerged when the comparing glucose injection data with the results from freeze-thaw. The liver multiplex data revealed that the PRX, SOD1 and TRX1 levels responded similarly to $200 \mathrm{mM}$ glucose as they did to freezing exposure, with decreased PRX and unaffected SOD1, TRX1 levels compared to the corresponding controls (Figs. 6.1A, 6.1B). This suggests that the high glucose produced and accumulated by liver during freezing exposure may be responsible for the regulation of these antioxidant enzymes in liver. However, the protein levels of catalase and SOD2 were uniquely suppressed only in the presence of high glucose, and not during 
freezing stress (Figs. 6.1A, 6.1B). Given that the expression of catalase and SOD2 genes is under Nrf2-dependent regulation (Kim and Vaziri, 2010), the liver multiplex data for glucose-loaded frogs are consistent with the gstal transcript levels (Fig. 6.8B, see below). These could be all be evidence of inhibition of Nrf2-related antioxidant defences in response to high glucose content. In terms of muscle, the multiplex results from glucoseloaded frogs matched those from the frogs experiencing freeze-thaw conditions (Figs. 6.2A, 6.2B), indicating high glucose content during freezing failed to introduce changes in the protein level of catalase, SOD1, SOD2 and TRX1 in muscle. However, the protein expression of PRX2 exhibited a different pattern where freeze-thaw stimulated its protein level while high glucose content led to a slight decrease (Figs. 6.2A, 6.2B). Overall, the multiplex results demonstrated that freeze-thaw conditions are able to alter the protein level of antioxidant enzymes. While consistent in muscle, the data also suggested that high glucose content might have unique responses to the combination of low temperature, oxygen deprivation and dehydration in frog liver when facing whole body freezing.

To further investigate the effect of glucose on antioxidant defense in wood frog, a commercially available assay kit was used to assess the overall antioxidant capacity after glucose injection. Relative antioxidant capacity was compared in liver and muscle between saline-loaded and glucose-loaded frogs. The data showed that the relative antioxidant capacity in liver of $2 \mathrm{~h}$ glucose-loaded frogs remained at a comparable level to that of saline injected frogs. However, in muscle, high glucose content boosted the antioxidant capacity by $\sim 3$-fold when compared with the saline injected frogs (Fig. 6.3). These results suggest antioxidant capacity is responsive to glucose levels in frog muscle but not in liver. Unchanged antioxidant capacity in the liver of glucose-injected frogs 
might result from the reduced protein levels of antioxidant enzymes observed in the multiplex assay. These results demonstrated that without the influence of subzero freezing body temperatures, the high glucose content was able to stimulate the antioxidant defense in the muscle of wood frog.

The study by Joanisse and Storey (1996) also demonstrated that the response of Nrf2-related antioxidant enzymes (i.e. GSTs) was part of the antioxidant defense associated with freeze tolerance in wood frogs. In fact, Nrf2-dependent gene expression was proposed to play a critical role in antioxidant defense in the freeze tolerant hatchling painted turtles as well as in hibernating mammals (Krivoruchko and Storey, 2010b; Morin et al., 2008; Ni and Storey, 2010). Our multiplex data showed regulation of the protein expression levels of various antioxidant enzymes that are downstream of Nrf2 (Fig. 6.1). In order to probe further into freezing-responsive Nrf2 regulation, the present study investigated the level of Nrf2 in liver and muscle of both frozen and glucose-loaded frogs. Immnoblotting results showed that the net protein levels of Nrf2 remained at control levels in both liver and muscle tissues of the wood frog after $24 \mathrm{~h}$ freezing exposure but increased upon $8 \mathrm{~h}$ thaw when compared with the frozen frogs (Fig. 6.4A).

Regulation on Nrf2 was also assessed in glucose-injected frogs. Frogs assessed at $2 \mathrm{~h}$ post-injection showed the same pattern of $\mathrm{Nrf2}$ protein in muscle as observed in frozen frogs (Figs. 6.4A, 6.4B). Both the Nrf2 protein and gstal transcript levels in muscle remained unchanged when compared to the saline-injected control frogs after $2 \mathrm{~h}$ glucose loading (Figs. 6.4B, 6.8B), consistent with the data from frozen frogs (Figs. $6.4 \mathrm{~A}, 6.8 \mathrm{~A})$. These data indicate that high glucose content failed to trigger changes in Nrf2-related antioxidant mechanisms with and without other freezing stress components. 
Therefore, with the protein levels of most Nrf2-related antioxidant enzymes unaffected by glucose-loading (Fig. 6.2B), the results suggest the high antioxidant capacity observed from glucose-loaded frogs may have been due to other antioxidant mechanisms.

Interestingly, the presence of high glucose stimulated the protein expression of Nrf2 in liver (Fig. 6.4B). However, transcript levels of gsta1, along with the protein levels of other Nrf2-dependent antioxidant enzymes (i.e. catalase, PRX2 and SOD2), dropped significantly in liver after glucose injection (Figure 6.1B,6.8B), suggesting that Nrf2 transcriptional activity was suppressed in the context of antioxidant defense. A recent study proposed that $\mathrm{Nrf2}$ is able to stimulate expression of genes encoding enzymes involved in glucose metabolism through the interaction with MafG (Hirotsu et al., 2012). Another study further revealed that Nrf2 promotes expression of enzymes that drive glycolysis and the pentose phosphate pathway (Mistsuishi et al., 2012). Therefore, it is possible that an increased level of Nrf2 observed in glucose-loaded liver was to direct excess glucose into either of these pathways of glucose use, since the cryoprotective function is unnecessary under the $5^{\circ} \mathrm{C}$ conditions of the glucose-injection experiments (Fig. 6.4B).

Despite the fact that the protein expression levels of Nrf2 were not affected in either liver or muscle during freeze-thaw (Fig. 6.4A), DNA binding activity by Nrf2 increased by about 1.5 -fold in both tissues after freezing exposure (Fig. 6.7B). Consistent with these data, the transcript levels of gstal were elevated in liver after $24 \mathrm{~h}$ freezing exposure (Fig. 6.8A), indicating increased Nrf2 transactivation activity in wood frogs experiencing freezing stress. Due to the accumulation of glucose during freezing exposure in wood frog, the present study also explored glucose effects on Nrf2 function 
by developing a different approach to DNA binding assays - directly assessing high glucose effects on Nrf2 binding in vitro. The in vitro results showed that $200 \mathrm{mM}$ but not $500 \mathrm{mM}$ glucose stimulated Nrf2 binding activity in both liver and muscle nuclear extracts of control frogs (Fig. 6.7C), suggesting a novel role of the cryoprotectant as a regulator of transcription factor binding activity in wood frogs during freezing.

These data indicate that the DNA binding activity of Nrf2 can be stimulated by glucose when equivalent to the plasma glucose content in wood frog during whole body freeze. The inconsistency between the in vitro DNA binding activity data and the gstal transcription level observed in both liver and muscle of the glucose-loaded frogs might be due to the subcellular glucose distribution states in those tissues at room temperature. Glucose uptake in wood frog during freeze exposure is dependent on a family of glucose transporters (GLUTs) (Pessin and Bell, 1992). Indeed, the level of membrane GLUT varies in wood frog seasonally. It was reported that the number of GLUT in liver membrane of autumn wood frogs was 8.5-fold higher than that of summer animals (King et al., 1995). Therefore, it is not a surprise that the Nrf2 transactivation activity of promoting gstal transcription didn't respond to glucose injection since low temperature appears to be a key factor affecting the efficiency of subcellular glucose distribution in wood frog. However, the liver data from the in vitro experiment were consistent with the elevated gstal transcript level upon $24 \mathrm{~h}$ freezing exposure (Fig. 6.8A). This suggests that the effect of high glucose content on Nrf2 transcription factor activity may not be instantaneous and only appeared after prolonged whole body freezing (24h). This, again, might be resulted from cell signal transduction and the membrane GLUT-dependent cellular glucose distribution (Storey and Storey, 2004a). 
Results from the ELISA-type transcription factor-DNA binding assay also suggested that the regulation of Nrf2 during freezing may be achieved by mechanisms other than solely modulating protein expression. The immunoblot data (Fig. 6.4) indicated a coordinated suppression of Nrf2 protein synthesis during freezing, agreeing with a general hypometabolic state under this energy-limited environmental stress. A potential candidate for regulating Nrf2 function might be post-translational reversible protein phosphorylation. It has been established that under circumstances of limited energy availability, it is more energy efficient to utilize posttranslational modifications such as reversible protein phosphorylation to control cellular processes (Storey and Storey, 2012). Indeed, recent studies proposed that Nrf2 is subject to regulation from GSK3 $\beta$-dependent phosphorylation events (Salazar et al., 2006; Jain and Jaisweal, 2007; Rada et al., 2011). Direct phosphorylation on Nrf2 by GSK3 $\beta$ promotes cytoplasmic sequestration of the transcription factor (Salazar et al., 2006) and its ubiquitin-dependent degradation (Rada et al., 2011). Also, an indirect mechanism was proposed contributing the GSK3ß-dependent Nrf2 inhibition (Jain and Jaisweal, 2007). GSK3 $\beta$ itself is also negatively regulated by phosphorylation. Therefore, the protein levels and phosphorylation status of GSK3 $\beta$ may be involved in the regulation of $\mathrm{Nrf} 2$ in wood frog tissues under freeze-thaw.

The immunoblotting data showed that the protein level of GSK3 $\beta$ dropped under both freeze and thaw conditions in liver, whereas the corresponding phosphorylated protein was elevated significantly upon freezing and returned to control level after $8 \mathrm{~h}$ thaw (Figs. 6.5A, 6.6A). This suggests that the overall phosphorylation level stayed high in liver during freezing. With both DNA binding activity of Nrf2 and gstal transcript 
levels increased in liver as well during freezing exposure, the elevated inhibitory phosphorylation level of GSK $3 \beta$ may contribute to $\mathrm{Nrf} 2$ activation. The Akt pathway is the main upstream kinase pathway responsible for GSK3 $\beta$ regulation. Upon activation, Akt phosphorylates and inhibits GSK3 $\beta$ (Pearl et al., 2002). The present observations about GSK $3 \beta$ agree with the previous studies that demonstrated that the Akt pathway was activated after $24 \mathrm{~h}$ freezing and remained active upon $8 \mathrm{~h}$ thaw in wood frog liver (Chapter 4). Furthermore, glucose-loaded frogs exhibited a strong increase ( $\sim .5$-fold) in GSK3 $\beta$ protein levels in liver while no appreciable changes were observed in relative phospho-GSK3 $\beta$ levels (as compared with saline-loaded frogs) (Figs. 6.5B, 6.6B), suggesting that high glucose content may have a positive regulatory effect on GSK3 $\beta$. This may explain the decreased catalase and SOD2 protein levels as well as gstal transcript levels in liver after glucose injection (Figs. 6.1B, 6.8B). Overall, the liver results suggest that a low body temperature may be required for Akt/GSK3 3 -dependent Nrf2 activation during freeze-thaw cycles, which is consistent with the previous observations that Akt activation was not observed in liver of dehydrated and anoxic wood frogs (Chapter 4).

In terms of muscle, the protein levels of GSK3 $\beta$ didn't show significant changes in response to freeze-thaw, whereas phosphorylation of the protein dropped in both frozen and thawed frogs compared to controls (Figs. 6.5A, 6.6A), suggesting inhibitory effects from Akt were relieved during freeze-thaw. Given that GSK3ß-dependent regulation of Nrf2 promotes cytoplasmic sequestration and protein degradation, it is possible that unchanged muscle gstal transcript levels were due to the combined effects of the freezing responsive increase of Nrf2 DNA binding activity (Fig. 6.7B) and the 
inhibitory regulation provided by GSK3 $\beta$ (Fig. 6.8A). A decrease in Akt-dependent phosphorylation of GSK3 3 in muscle also agrees with the previous observation that the pathway was inhibited under freeze-thaw conditions (Chapter 4). Similar to freeze-thaw exposure, high glucose content didn't affect the protein level of GSK3 $\beta$ but induced a decrease in the phosphorylation state (Figs. 6.5B, 6.6B). Again, with increased DNA binding activity of Nrf2 exhibited from the in vitro assay and GSK3 $\beta$-dependent inhibitory regulation, the transcript level of gstal in the muscle of the glucose-loaded frogs remained at a comparable level to the saline injected animals (Fig. 6.8B). The similar responses of GSK3 $\beta-\mathrm{Nrf2}$ in the muscle of the glucose-loaded frogs to frozen frogs suggest that glucose (not temperature) may be a key factor behind the muscle response of GSK3 3 -Nrf2.

As one of the Nrf2 downstream genes, one main function of GST is to protect cells against damage caused by electrophiles produced from peroxidation of membrane polyunsaturated fatty acids by free radicals via GSH conjugation and peroxidase activity (Hayes et al., 2005). Since liver membrane holds a crucial position for glucose transportation in wood frogs (Pessin and Bell, 1992), it is of great importance to protect it from oxidative damage during freeze-thaw cycles. Therefore, it is perhaps not a surprise that the protein expression level of Nrf2 was elevated in both tissues after thawing. However, DNA binding activity of Nrf2 dropped in liver but retuned to the control level in muscle upon thawing (Fig. 6.7B). The transcript levels of gstal did not change compared to control animals in either tissues after $8 \mathrm{~h}$ thaw. These results agree with the previous study that the most antioxidant enzymes were not activated upon thawing (Joanisse and Storey, 1996). Combined with the multiplex data, the activation 
of Nrf2 in muscle upon freezing exposure appears to stimulate only selected gene/proteins including PRX2 but not GSTa1 (Figs. 6.2A, 6.7B, 6.8A) (Li et al., 2010), suggesting a differential regulatory effect from Nrf2.

In conclusion, the present study examined the antioxidant response of wood frogs experiencing whole body freezing and thawing. The study confirms and further explores the findings by Joanisse and Storey (1996) by investigating protein expression levels of major antioxidant enzymes (Figs. 6.1, 6.2), the overall antioxidant capacity (Fig. 6.3), and Nrf2-dependent antioxidant defense mechanisms. Due to the fact that glucose is accumulated in wood frog as the cryoprotectant during freezing exposure, the role of glucose in modulating antioxidant defense responses in wood frog organs was also investigated. The multiplex assay suggested that the presence of high glucose content generally did not affect the protein levels of antioxidant enzymes in muscle but had inhibitory effects in liver. However, high glucose content led to an increased overall antioxidant capacity in muscle. The Nrf2-dependent antioxidant pathway also showed responses to glucose loading in both tissues. Generally, high glucose content promoted the expression levels of Nrf2 protein and Nrf2 DNA binding activities. Data from muscle suggest that high glucose content has the same effects with and without other components of freezing stress (i.e. low temperature, dehydration and oxygen deprivation). However, Nrf2 might play a different role (other than antioxidant defense) in the response to high glucose injection in liver. For example, the glucose-induced activation of Nrf2 in liver did not lead to enhanced transcription of gstal. This might be due altered cell signal transduction and glucose transportation. For example, in liver, a glucose-dependent increase of Nrf2 DNA binding activity was only reflected in the gstal transcript levels of 
frozen frogs but not glucose-loaded animals. Previous studies have proposed a difference in the level of membrane glucose transporters in liver between autumn and summer frogs, strongly suggesting a role of temperature in glucose distribution (King et al., 1995). GSK3 $\beta$ was also assessed as a regulator of Nrf2, which also links to Akt signaling. The Akt-dependent phosphorylation status of GSK3 $\beta$ was consistent with the findings earlier in this thesis that the Akt pathway was activated in liver but not in muscle of wood frogs under freeze-thaw conditions. It also appears that low temperature may be required for the liver Akt-GSK3 $\beta-\mathrm{Nrf} 2$ regulation whereas glucose may be more responsible than low temperature for the muscle responses during freeze-thaw cycles. In conclusion, the present study demonstrates that glucose-related Nrf2-dependent antioxidant defense is an integral part of the freeze tolerance by wood frogs. Furthermore, the study reported a novel finding that high glucose content $(\sim 200 \mathrm{mM})$ is able to promote transcript factorDNA interactions, potentially providing a way for this simple sugar to effectively modify the action not only of Nrf2 but potentially of numerous other transcription factors. 
Table 6.1A. Plasma glucose levels $(\mathrm{mM})$ after saline or glucose injections. Data are means \pm SEM, $n=6$ independent determinations from different animals.

\begin{tabular}{lllll}
\hline Groups & Control & 2h Saline & 2h Glucose & 6h Glucose \\
\hline $\operatorname{Mean} \pm \operatorname{SEM}(\mathrm{mM})$ & $0.74 \pm 0.14$ & $0.71 \pm 0.17$ & $191.0 \pm 8.74$ & $177.7 \pm 11.5$ \\
\hline
\end{tabular}

Table 6.1B. Glucose assay kit interference test (STD: standard solution)

\begin{tabular}{lll}
\hline & STD $(\mathrm{mM})$ & $\mathrm{STD}+(1 / 50) 6 \mathrm{~h}$ Glucose $(\mathrm{mM})$ \\
\hline Theoretical & 0.69 & 2.12 \\
Measured & 0.61 & 2.06 \\
\hline
\end{tabular}


Fig. $6.1 \mathrm{~A}$

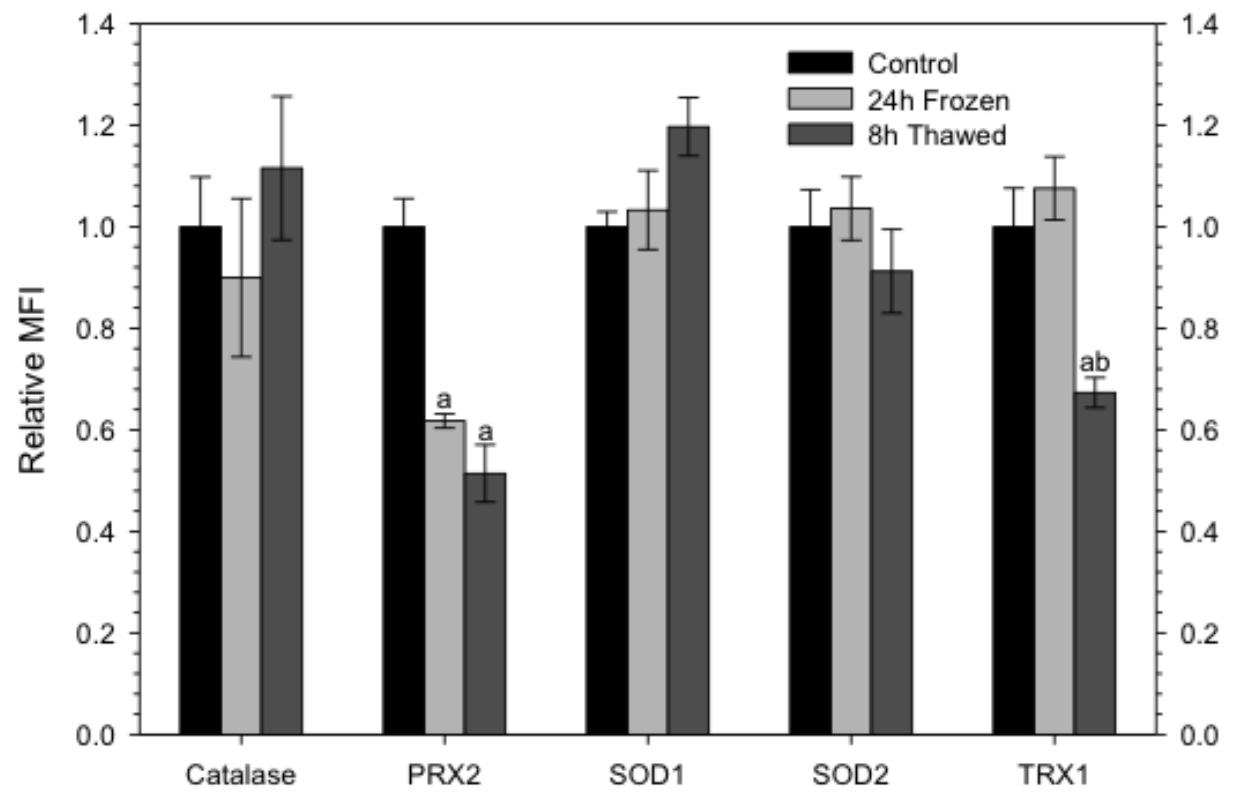

Fig. 6.1B

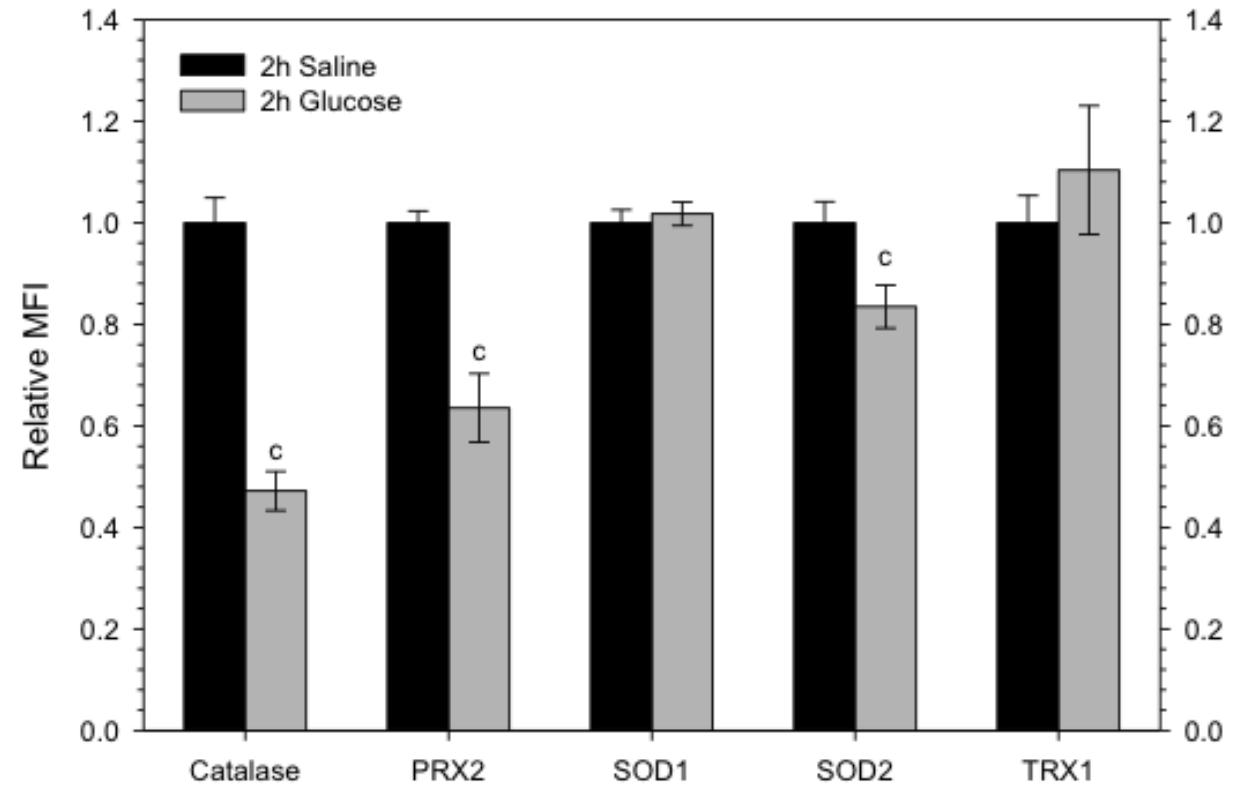

Fig. 6.1. Effects of freeze-thaw (A) and glucose injection (B) on total protein levels of catalase, PRX2, SOD1, SOD2 and TRX1 in wood frog liver. Data are mean \pm SEM, n=34 independent trials on tissue from different animals. a- significantly different from the corresponding control group, b-significantly different from the corresponding frozen group, c-significantly different from the corresponding saline-loaded group $(\mathrm{P}<0.05)$. 
Fig. $6.2 \mathrm{~A}$

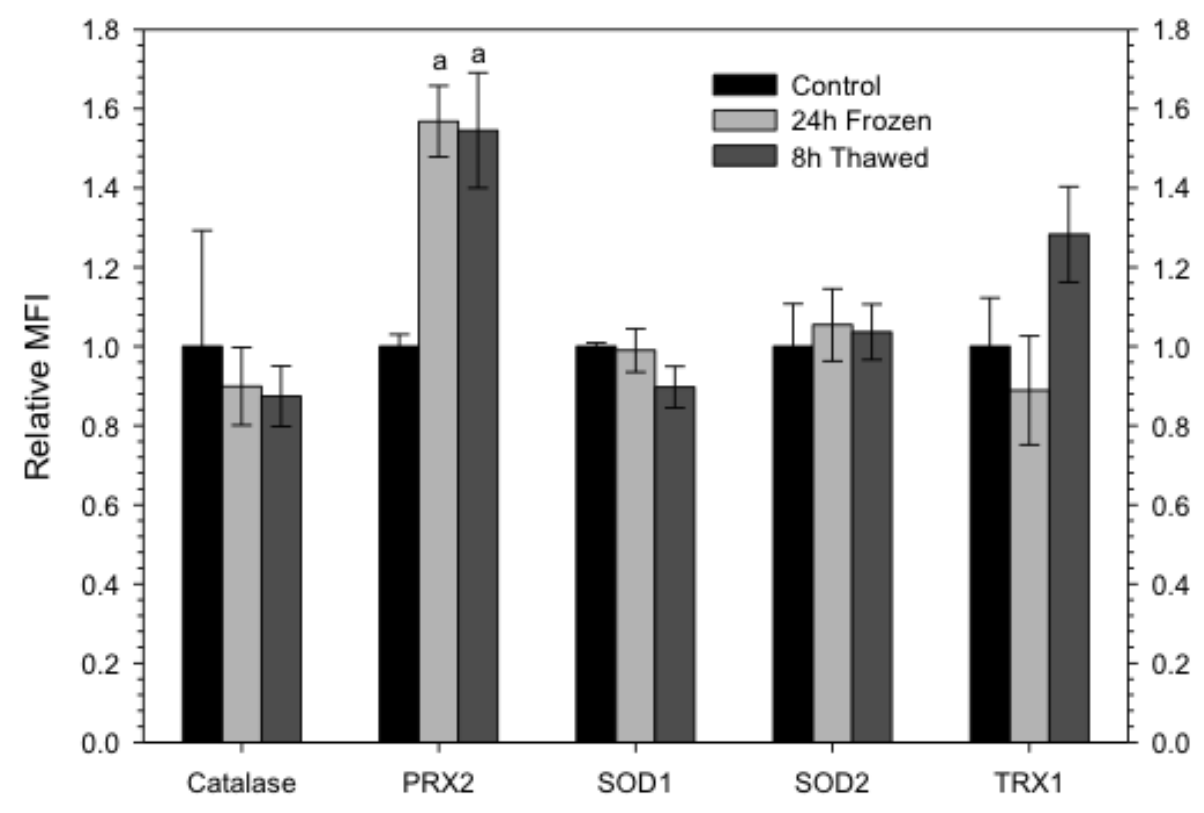

Fig. 6.2B

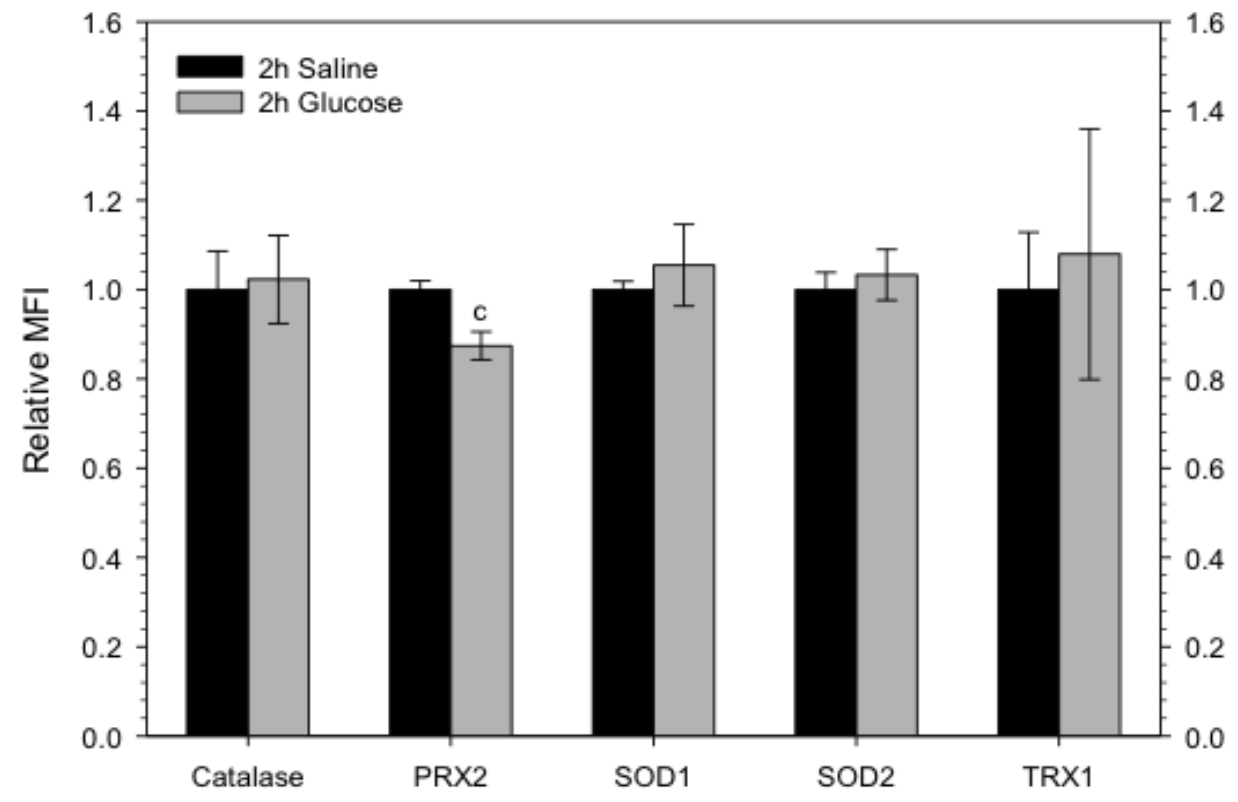

Fig. 6.2. Effects of freeze-thaw (A) and glucose injection (B) on relative total protein levels of catalase, PRX2, SOD1, SOD2 and TRX1 in wood frog skeletal muscle. Other information as Fig. 6.1. 
Fig. 6.3

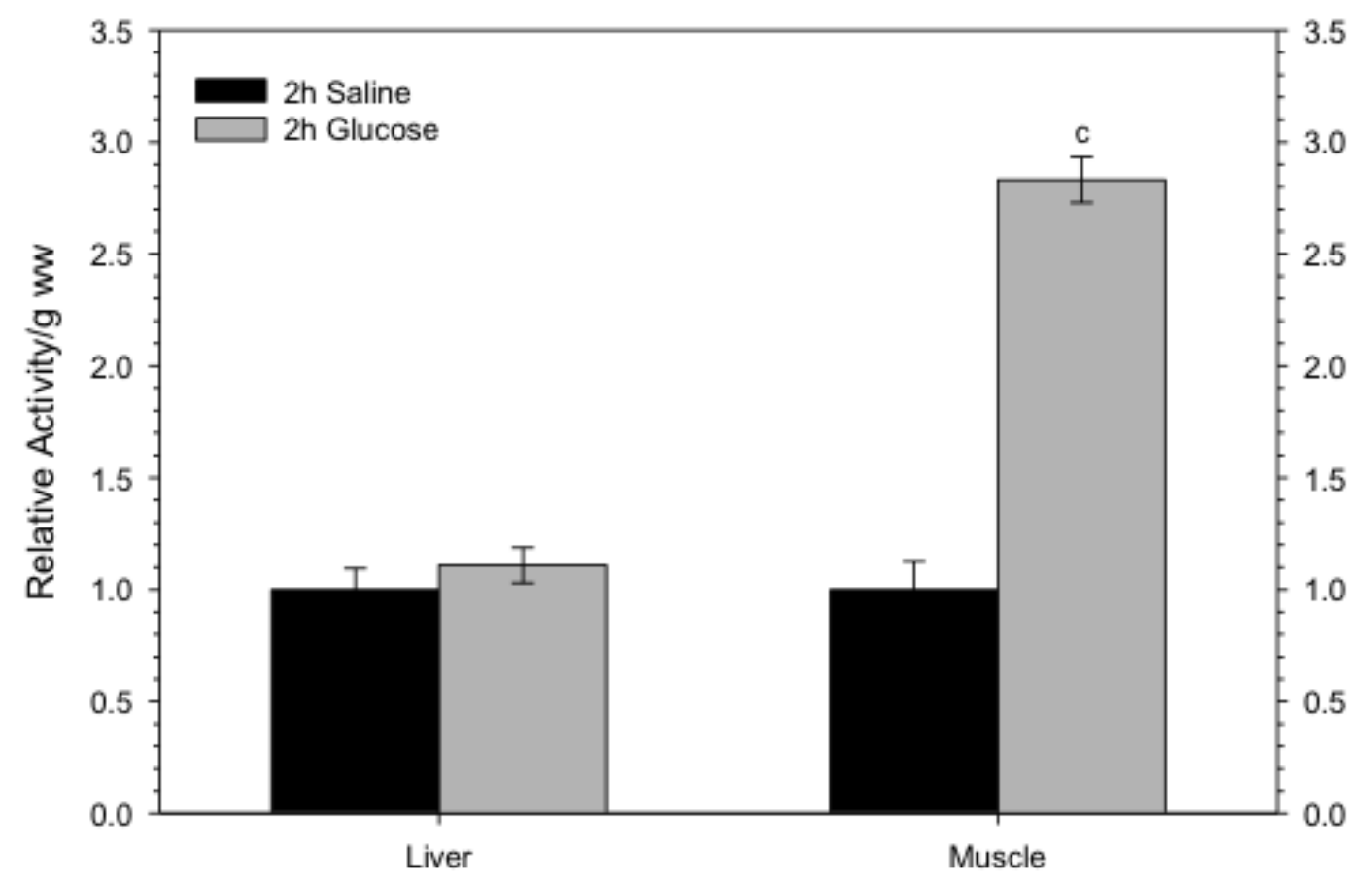

Fig. 6.3. Effects of glucose injection on antioxidant capacity in liver and muscle of wood frogs. Data are mean $\pm \mathrm{SEM}, \mathrm{n}=3-4$ independent trials on tissue samples from differentanimals. a- significantly different from the corresponding control group, csignificantly different from the corresponding saline-loaded group $(\mathrm{P}<0.05)$. 
Fig. 6.4A
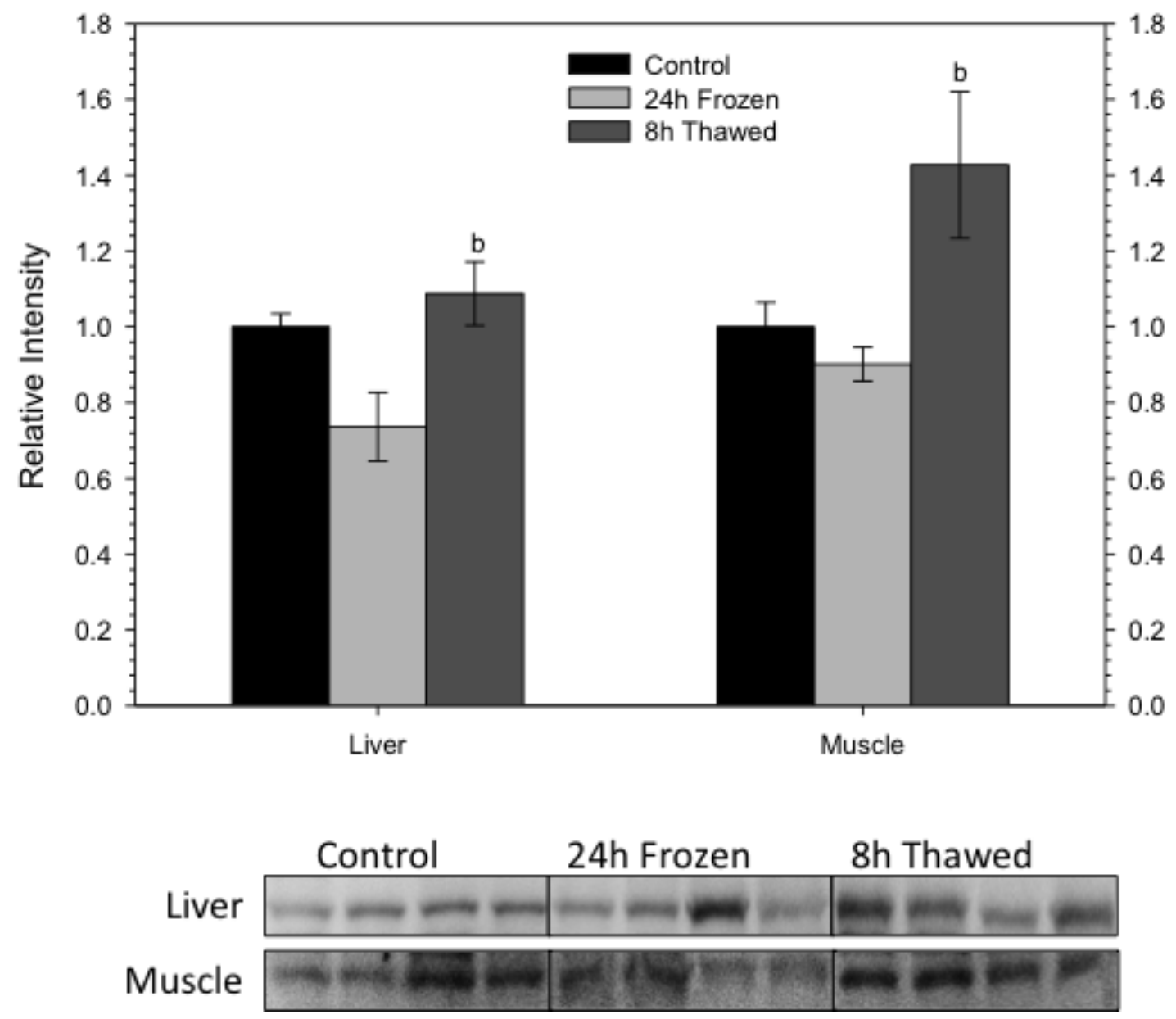
Fig. 6.4B
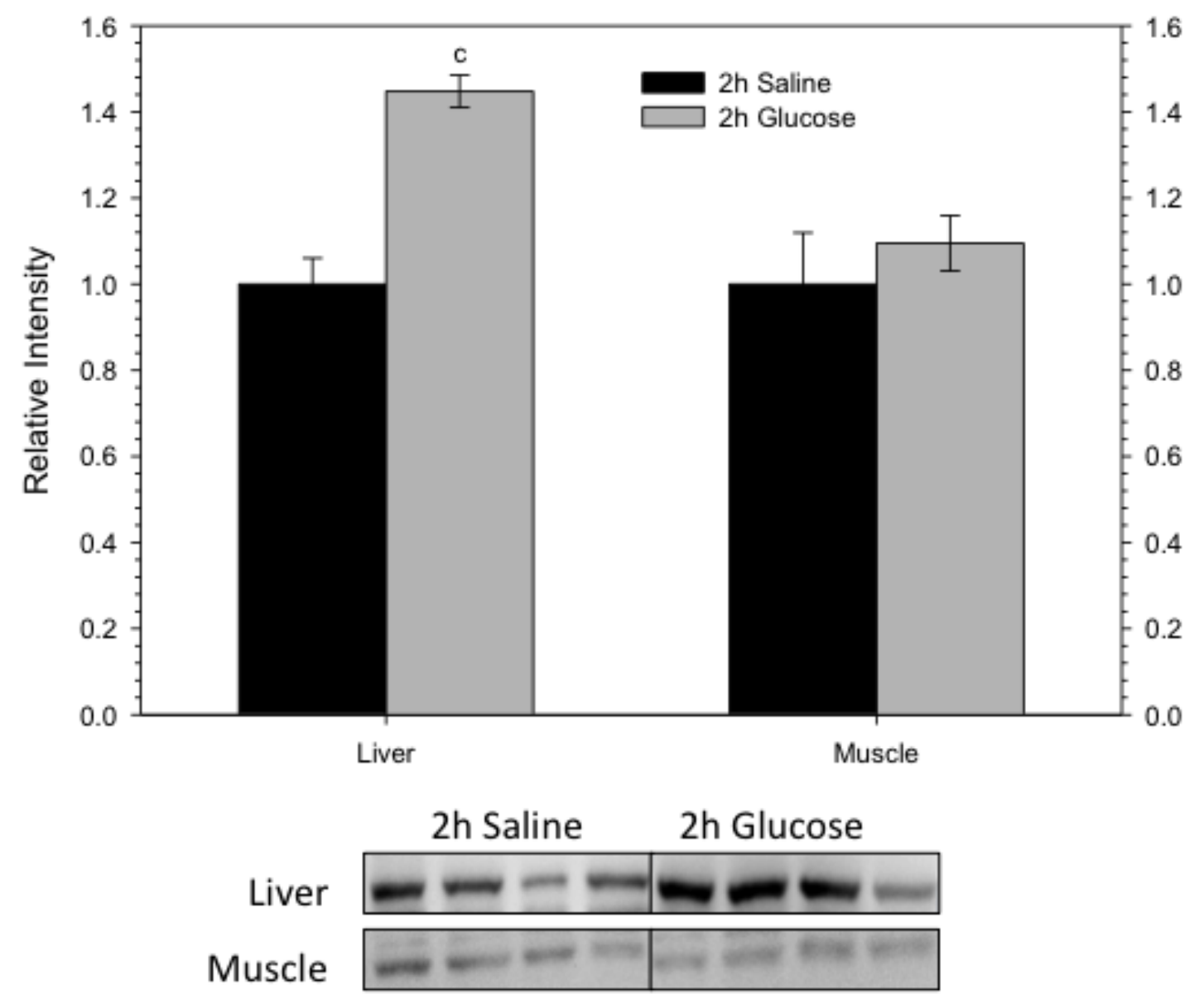

Fig. 6.4. Effects of freeze-thaw (A) and glucose injection (B) on relative total protein levels of Nrf2 in wood frog liver and skeletal muscle. Upper portion shows the histogram showing standardized relative protein levels; lower portion shows representative immunoblotting bands of Nrf2. Data are mean \pm SEM, $n=3-4$ independent trials on samples from different animals. a- significantly different from the corresponding control group, b-significantly different from the corresponding frozen group, c-significantly different from the corresponding saline-loaded group $(\mathrm{P}<0.05)$. 
Fig. $6.5 \mathrm{~A}$
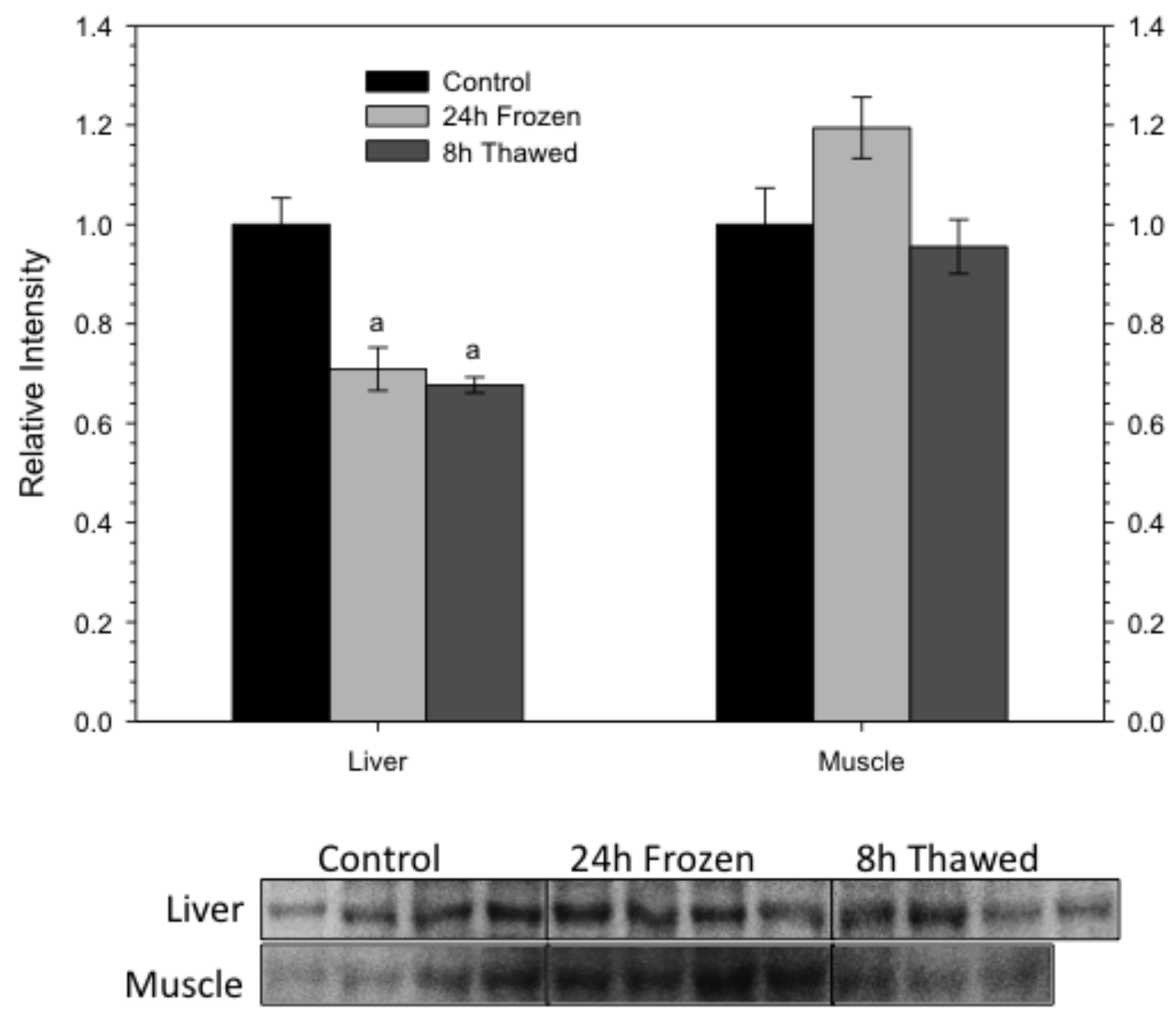
Fig. 6.5B

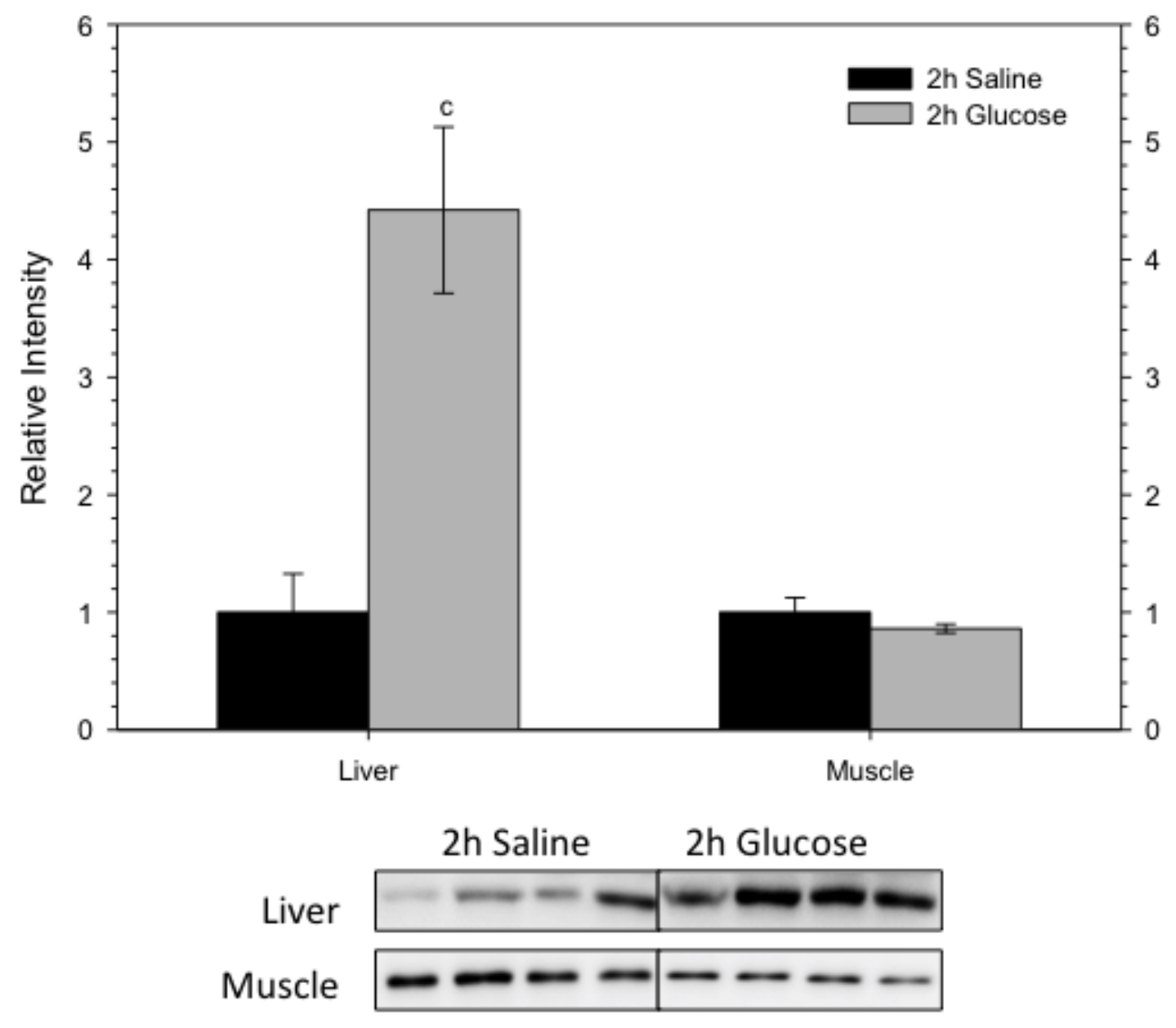

Fig. 6.5. Effects of freeze-thaw (A) and glucose injection (B) on total protein levels of GSK3 $\beta$ in wood frog liver and muscle. Upper portion is the histogram showing standardized relative protein levels; lower portion shows representative immunoblotting bands of GSK3 $\beta$. Other information as Fig. 6.4. 
Fig. $6.6 \mathrm{~A}$
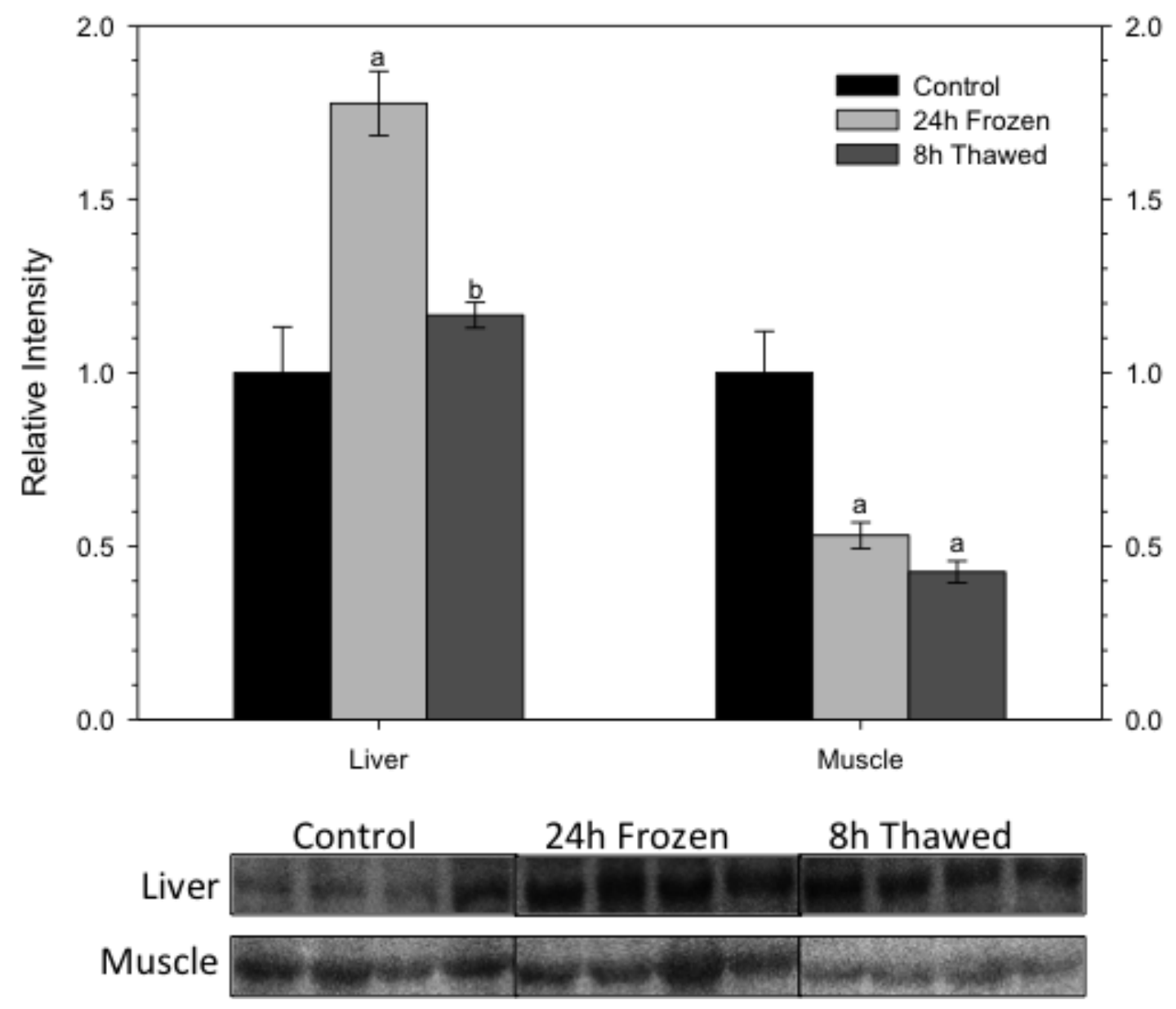
Fig. 6.6B

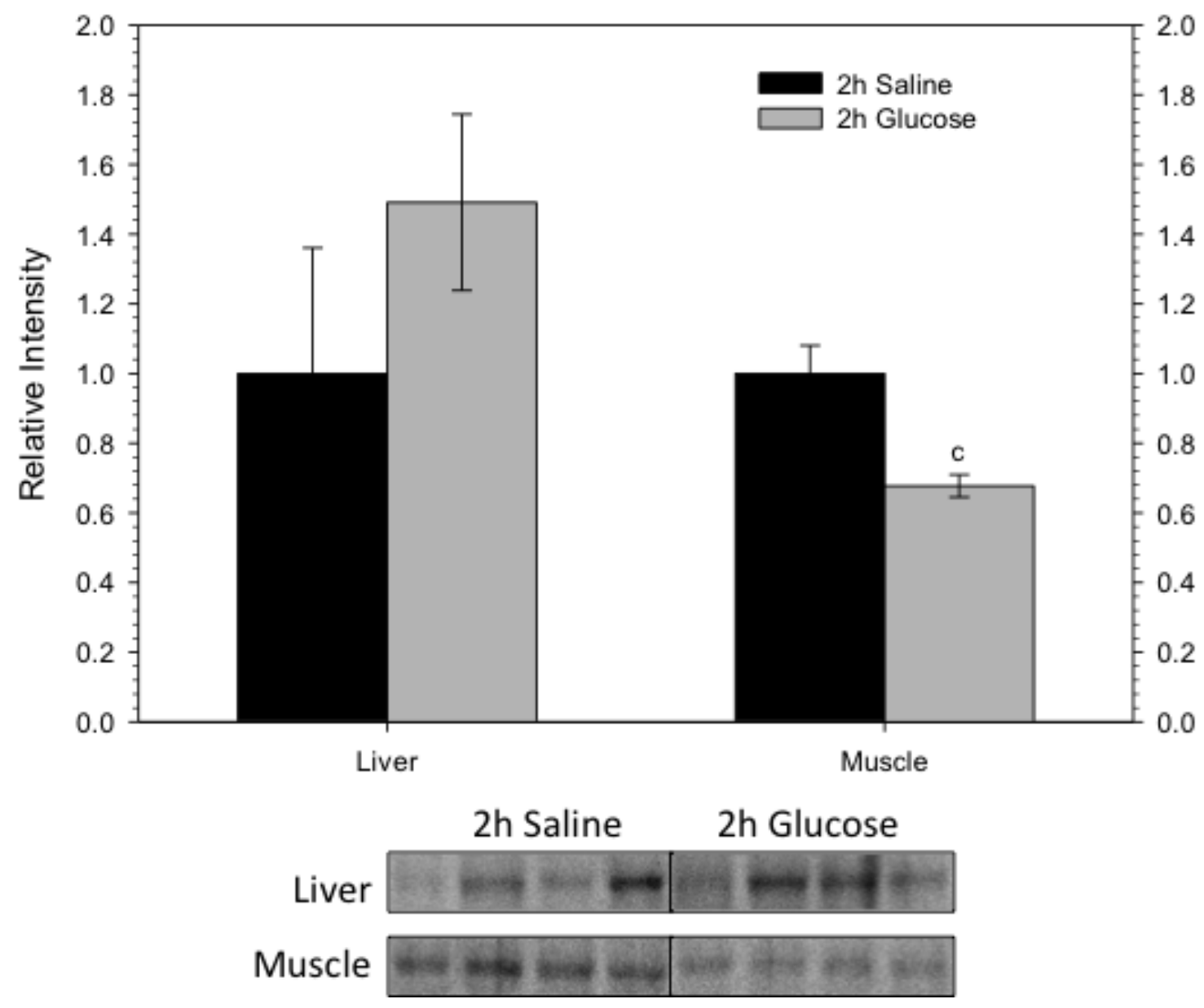

Fig. 6.6. Effects of freeze-thaw (A) and glucose injection (B) on the relative amount of phospho-GSK3 $\beta$ (Ser-9) detected in wood frog liver and muscle. Upper portion is the histogram showing standardized relative protein levels; lower portion shows representative immunoblotting bands for phospho-GSK3 $\beta$ (Ser-9). Other information as Fig. 6.4. 
Fig. 6.7A

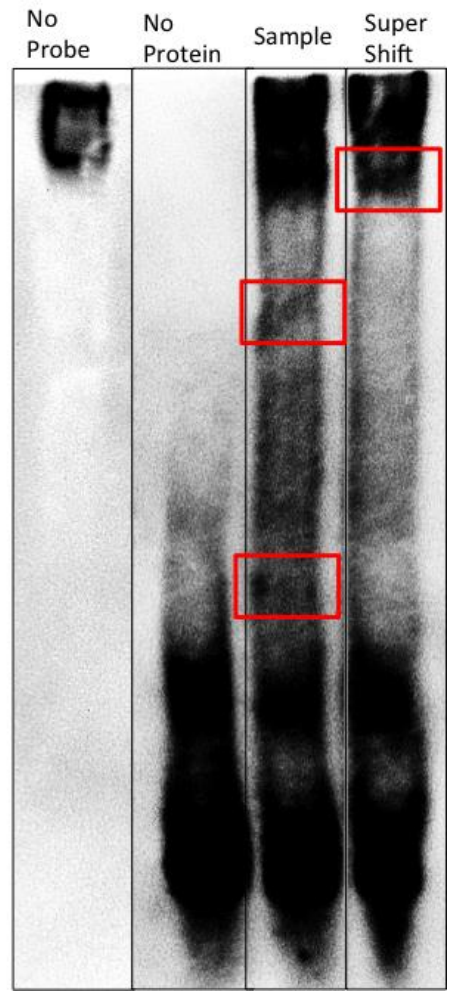

Fig. 6.7B

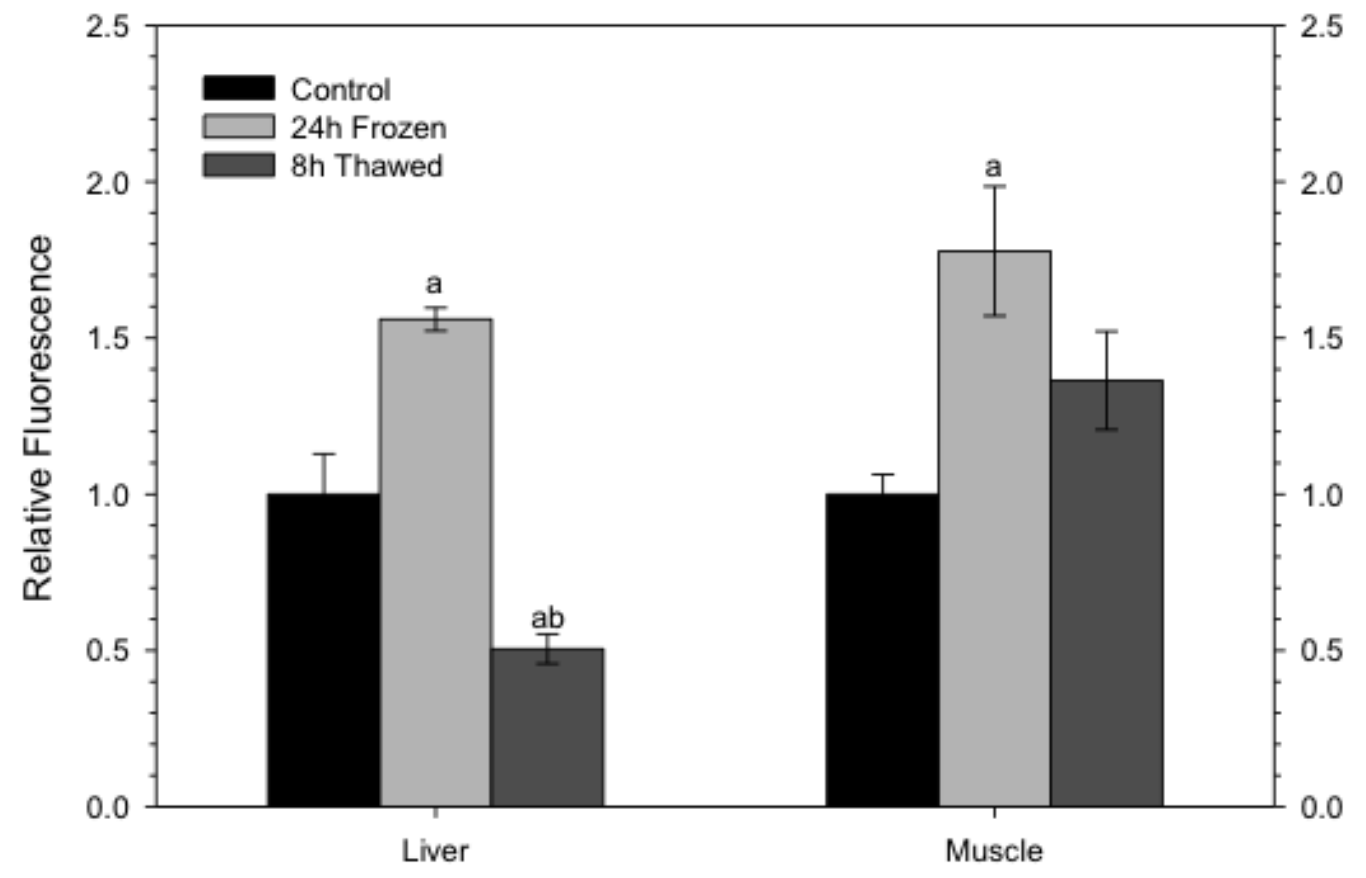


Fig. 6.7C

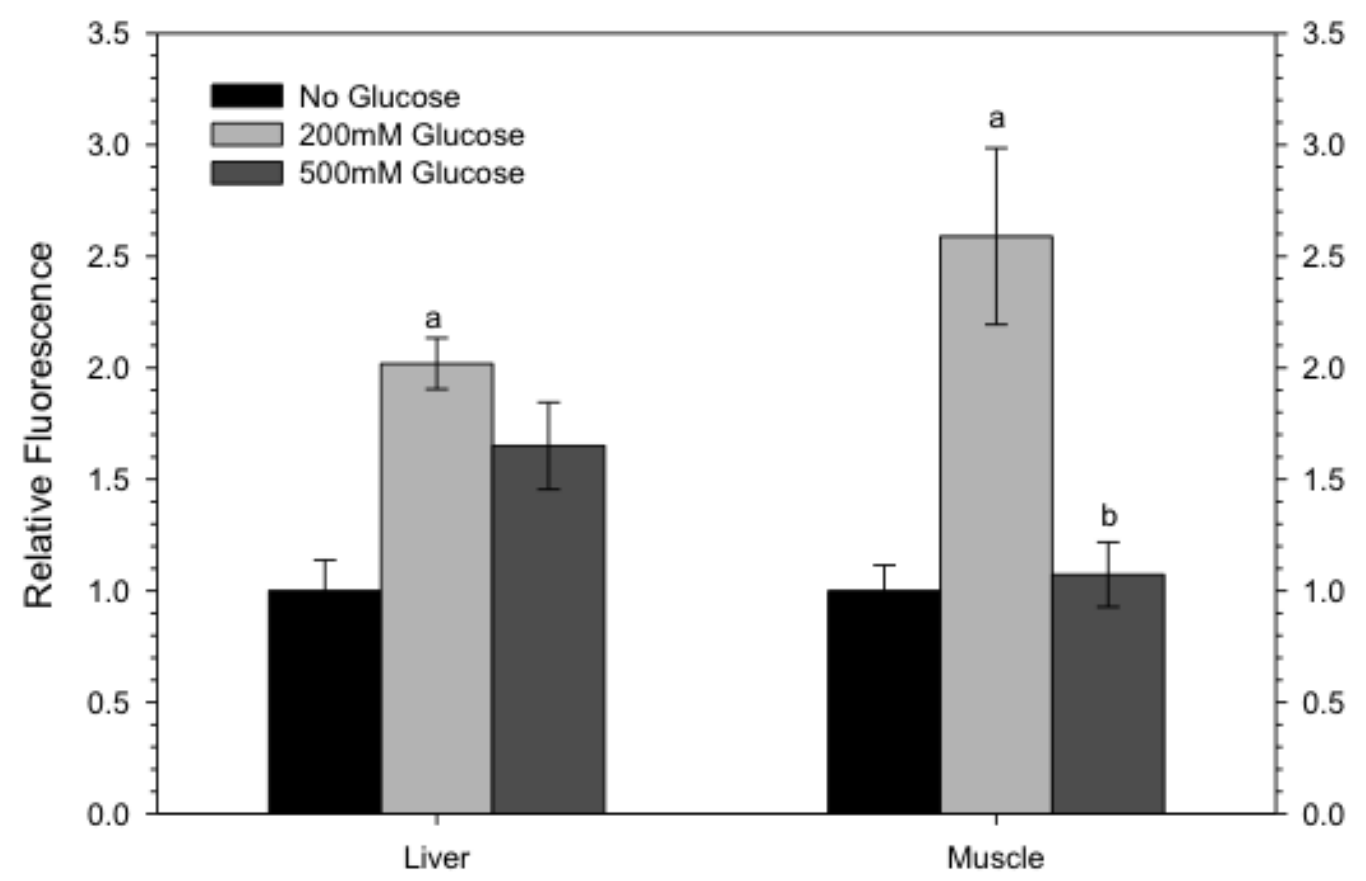

Fig. 6.7. EMSA and ELISA-type transcription factor-DNA binding assays. (A) EMSA shift picture showing Nrf2-ARE probe interactions. First and second lanes are no probe and no protein controls while third and fourth lanes show sample and supershift conditions, respectively. The boxes indicate the bands of interest.

(B) Effects of freeze-thaw on Nrf2-ARE binding activity by extracts of wood frog liver and muscle as detected with ELISA assays. (C) Effects of $200 \mathrm{mM}$ or $500 \mathrm{mM}$ glucose on Nrf2-ARE binding activity in wood frog liver and muscle in ELISA assays. Data are mean \pm SEM, $n=3-4$ independent trials on tissue samples from different animals. asignificantly different from the corresponding control or no glucose group, b-significantly different from the corresponding frozen or $200 \mathrm{mM}$ glucose group $(\mathrm{P}<0.05)$. 
Fig. $6.8 \mathrm{~A}$
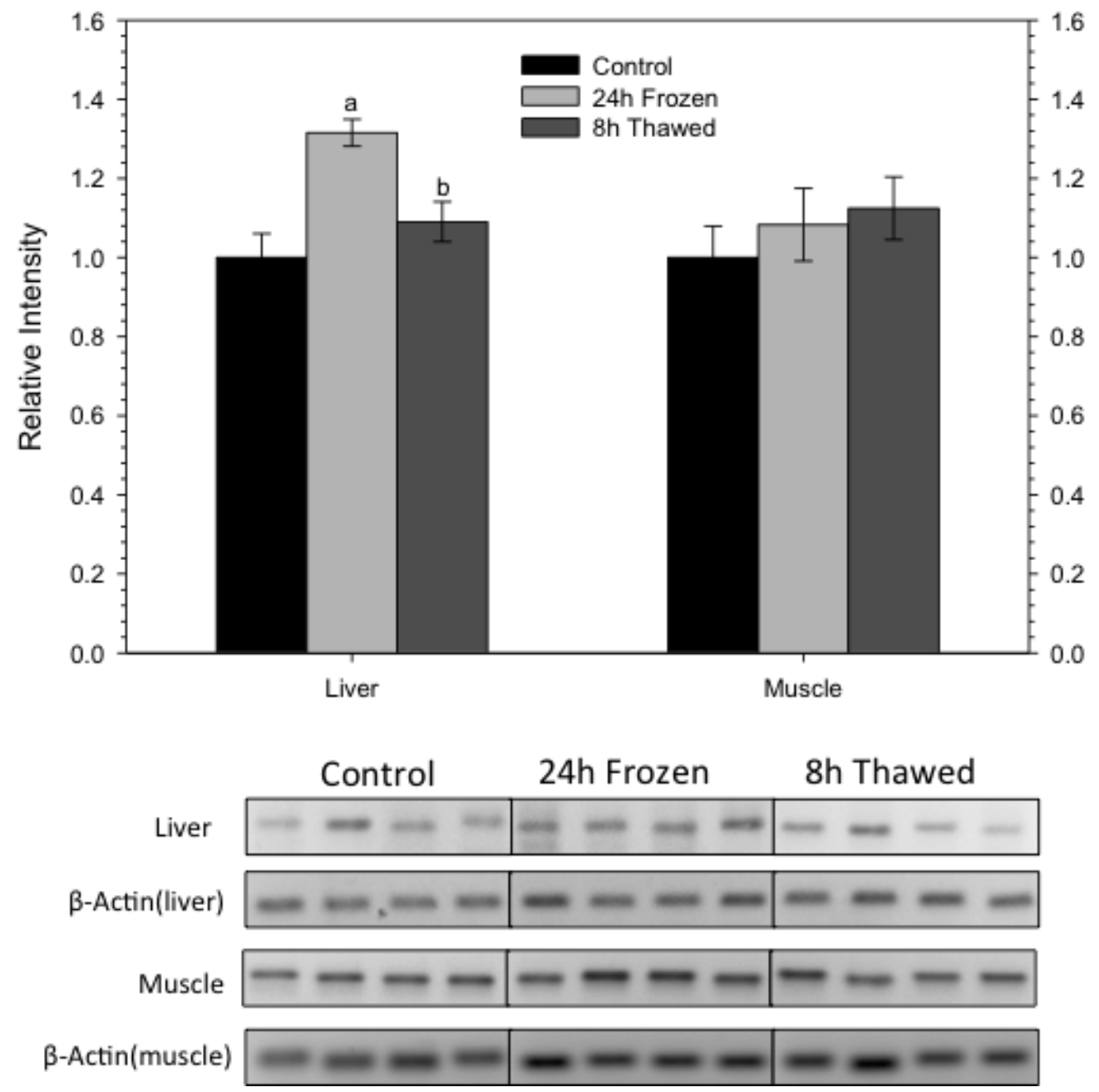
Fig. $6.8 \mathrm{~B}$
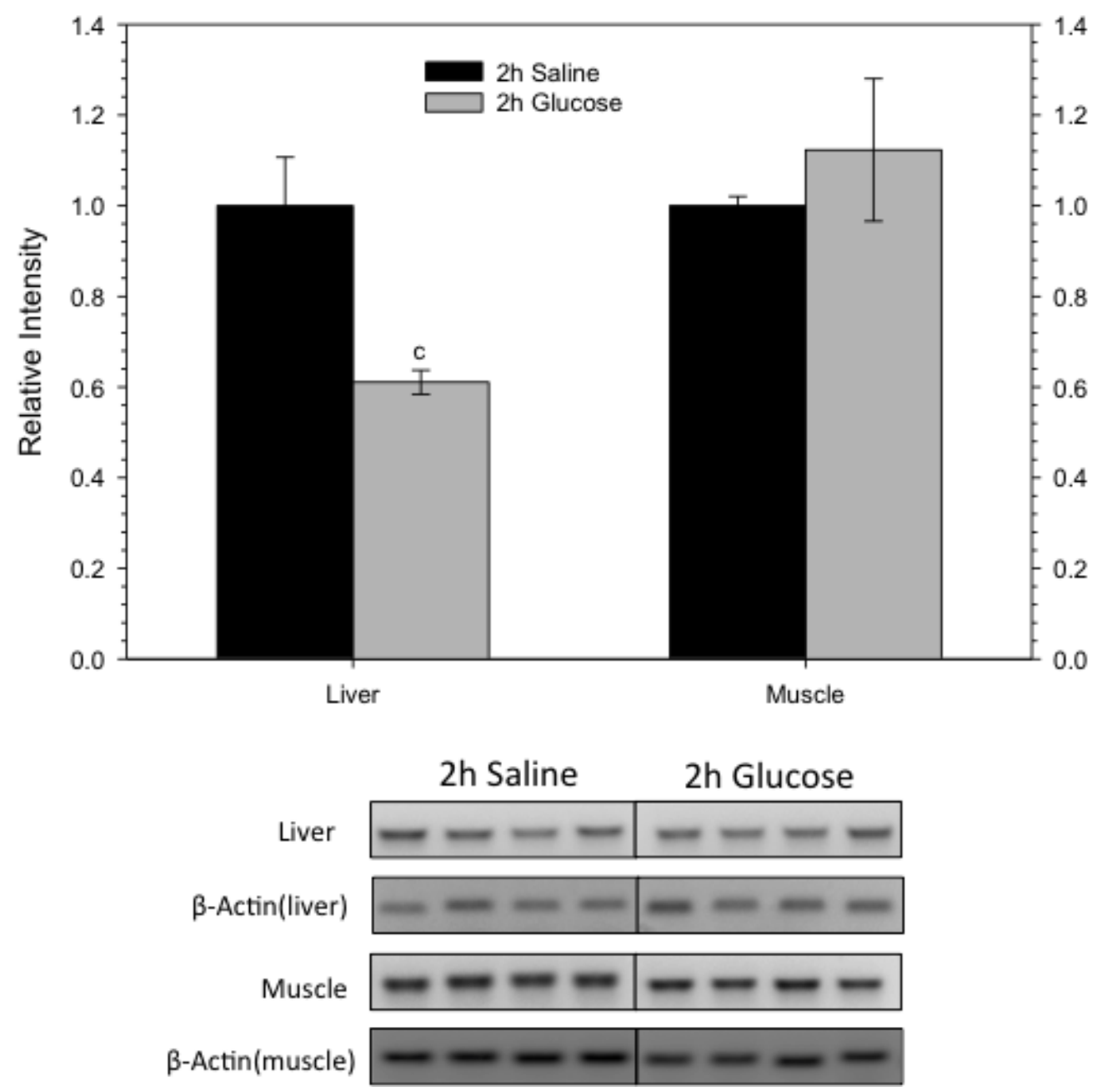

Fig. 6.8. Effects of freeze-thaw (A) and glucose injection (B) on transcript level of gstal in wood frog liver and muscle. Upper portion is the histogram showing standardized relative transcript levels; lower portion shows bands for gstal and $\beta$-actin amplicons. Data are mean \pm SEM, $n=3-4$ independent trials on tissue samples from different animals. a- significantly different from the corresponding control group, b-significantly different from the corresponding frozen group, c-significantly different from the corresponding saline-loaded group $(\mathrm{P}<0.05)$. 


\section{Chapter 7 General discussion}




\section{Chapter 7 - General Discussion}

Hundreds of species of animals experience seasonal cold during their life span. Freezing is extremely harmful to organisms that lack without proper freezing survival adaptations. Damage from freezing stress can take multiple forms such as physical injury to tissues, interruption of fundamental vital processes (i.e. heartbeat, breathing), osmotic stress on cells caused by the high extracellular osmolality, damage to membranes due to cell shrinkage, and oxidative stress caused by ischemia-reperfusion, among others. When challenged by subzero temperature exposure, most organisms find a way to avoid the cold or to endure the cold by supercooling. However, some organisms have developed the capability of natural freeze tolerance. Freeze tolerance is typically defined as the ability to endure internal body ice formation in extracellular spaces with equilibrium ice contents of typically $50-70 \%$ of body water and under ecologically relevant conditions of freeze duration and temperature conditions that naturally occur in the winter microhabitat of the species (Costanzo et al., 1993; Layne, 1995a). Freezing tolerance has been widely adopted by a variety of animal species including several frog species and cold hardy insects (Storey and Storey, 2004a; Storey and Storey, 2012)

During freezing exposure, freeze tolerant organisms enter a state where normal physiological processes (e.g. breathing, muscle movement, heartbeat, etc.) are interrupted or suppressed, eventually leading to a situation where every cell in the body must survive as a self-contained unit with its own fuel supply and cut off from oxygen uptake or the ability to export wastes. With restricted reserves of fermentable fuel and rapidly declining oxygen availability, freeze tolerant organisms are usually confronted by limited ATP production and turn to metabolic rate depression to reduce energy needs in the frozen 
state and achieve long term cell/tissue preservation. Therefore, a main theme of freeze tolerance is to establish new equilibrium between energy output and consumption, or to reprioritize energy expenditure. A crucial strategy for achieving this is to suppress or stop energy expensive yet optional cellular events and to dedicate the limited energy pool to stress responsive adaptations.

Because of their well-developed natural freeze tolerant adaptations, wood frogs $(R$. sylvatica) have been used as a primary animal model for studies of vertebrate freezing survival. The physiological and biochemical aspects of the wood frog freezing tolerance have been established over many years of research (Storey and Storey, 2004a). With the recent addition of high throughput techniques in molecular biology such as DNA microarray and LC-MS, considerable data is accumulating about the expression and regulation of genes and proteins that are involved in freezing preservation (Storey, 2004b; Kiss et al., 2011). Based on these findings, the goal of my thesis was to explore the role of the Akt stress responsive signal transduction pathway and the response/regulation of cellular events that are under Akt control (e.g. cell cycle, protein synthesis) in wood frogs to determine how these contribute to cell preservation in the frozen state. In addition, novel aspects of cryoprotection by glucose were explored including glucose effects on the functioning of the Nrf2 transcription factor and on the expression of antioxidant enzymes. A variety of techniques have been used in the thesis, including immunoblotting, RT-PCR, EMSA, ELISA-type transcription factor-DNA binding assay, Luminex-based multiplex assay, and several specialized enzyme activity assay kits (see Chapter 2 for details). 


\section{Molecular mechanisms of freeze tolerance by wood frog}

Like other freeze tolerant animals, both basal metabolism and stress responses need to be supported by restricted options for generating energy in the frozen state (chiefly anaerobic glycolysis). Thus, suppression of energy-expensive processes including cell proliferation and cell growth is expected. In addition, due to the unique adaption of accumulating high glucose content in wood frog, pathways related to glucose production in liver needed to be specifically regulated upon freeze-thaw. Therefore, tissue-specific responses under freezing stress are also expected. However, these cannot be achieved without a proper stress responsive signaling network, as suggested by both DNA microarray and LC-MS studies (Storey, 2004b; Kiss et al., 2011).

Therefore, my thesis started by examining the regulation of the cell cycle. I explored a signaling pathway that was potentially responsible for regulating both the cell cycle and high glucose levels: the Akt pathway. Furthermore, since the Akt pathway is a major regulator of protein synthesis (another energy expensive process,) via the TORC1 pathway, the thesis also investigated the regulation and responses of the TORC1 pathway. Ultimately, extra cryoprotective roles of glucose in the context of Nrf2-related antioxidant defense were also explored.

\section{Cell cycle suppression in liver}

As described above, cell proliferation is one of the "optional" metabolic processes that are hypothesized to be suppressed during hypometabolism. Investigating the protein expression level and phosphorylation status of the key factors of cell cycle is one way to 
examine freezing responsive regulation of cell proliferation. The cell cycle is primarily driven by a set of Cdk-cyclin complexes. The two most important regulatory periods are the G1/S phase transition and the G2/M phase transition. For the G1/S transition in eukaryotic cells, Cdk 2, -4, -6 with cyclins A, -D and -E play crucial roles, whereas Cdc2 (also known as Cdk1 in metazoans) and cyclins A, -B1, -B2 are vital to the G2/M phase transition. The Cdc2/Cyclin A (-B) complex is also called the M-phase Promoting Factor (MPF), with the function of driving the cell cycle into M phase (Harashima et al., 2013). The activity of most regulatory $\mathrm{Cdk} /$ cyclin complexes is related to their phosphorylation state. Inhibitor proteins called Cdk inhibitors (CdkIs) are also important, which include the $\mathrm{KIP} / \mathrm{CIP} \mathrm{Cdk}$ inhibitors $\mathrm{p} 21$ and $\mathrm{p} 27$. In addition to freezing conditions, it is also believed that hypometabolism in wood frogs occurs in response to two of the component stresses of freezing: cellular dehydration and anoxia stresses (Storey and Storey, 2004a). Therefore, I hypothesized that expression of key cell cycle components would be suppressed and/or regulated in favour of cell cycle arrest under freezing, dehydration or anoxia stresses in wood frog liver, potentially showing stress-specific patterns. Immnoblotting was used to investigate the protein expressions of key cell cycle regulators including $\mathrm{Cdc} 2, \mathrm{Cdks}(2,4,6)$, Cyclins (A, B1, D1, E) and phosphorylation levels of Cdk (Thr-14/Tyr15), phosphatases [Cdc25a (Ser-76) and Cdc25c (Ser-216)] and CdkIs [p21 (Thr-145) and p27 (Thr-187)] in wood frog liver after $24 \mathrm{~h}$ freezing, $24 \mathrm{~h}$ anoxia and $40 \%$ dehydration exposures, as well as the corresponding recovery conditions.

The overall trend showed that the protein expression level of most Cdks and cyclins dropped under freezing exposure and most returned to control levels upon thawing (Figs. 3.2 - 3.7), suggesting cell cycle suppression. Stress specific effects were 
also discovered for certain Cdks and cyclins (Figs. 3.2 - 3.7). For example, anoxia and dehydration exhibited stronger inhibitory effects on the protein levels of most Cdks and cyclins than freezing exposure, indicating a possible stronger inhibition in liver under anoxic and dehydrated conditions. The overall inhibitory phosphorylation level Cdks also increased significantly in response to anoxia and dehydration but during freezing remained at a comparable level as control animals (Figs. 3.2-3.4), providing another piece of evidence of stronger cell cycle inhibition upon anoxia and dehydration exposure than freezing. This is also consistent with the observation of inhibition of the phosphatase Cdc25c in response to anoxia and dehydration but not freezing (Fig. 3.8), since Cdc25c activates Cdks by dephosphorylation (Karlsson-Rosenthal and Millar, 2006). Relative protein level of cyclins also showed a similar pattern as for Cdks. The level of all cyclins assessed dropped substantially in liver under anoxia and dehydration pressure when compared to control values; and again, liver from frozen frogs exhibited less inhibitory effects on expression levels of cyclins (Figs. 3.5-3.7). While cyclin A and B1 levels dropped significantly after freezing exposure, cyclin E failed to show changes in protein levels in liver. This suggests that freezing stress may mainly impact the later stages of cell cycle progression, given that the Cdk2-cyclin E complex drives the G1/S transition and DNA synthesis. Interestingly, the protein level of cyclin D1 increased after $24 \mathrm{~h}$ freezing exposure in wood frog liver. Since cell cycle progression is based on the various complex assemblies, increased protein levels of one particular factor usually won't impact the overall trend of the regulation on cell cycle. Notably, Cyclin D1 has been proposed to also act as a transcription factor that regulates processes in addition to the cell cycle (Bienvenu et al., 2010). As a transcription factor, cyclin D1 has been shown to 
promote expression of the gene encoding the Notch1 transmembrane receptor, which is tied closely to hepatic glucose production (Pajvani et al., 2011) Given the importance of glucose to freeze tolerance by wood frogs, it is not surprising therefore that the cyclin D1 showed a different pattern of expression during freezing as compared with other cyclins. Although not pursued further in the present thesis, it would clearly be valuable to explore the links between cyclin D1 and freezing-responsive glucose production since there must be a molecular mechanism that allows wood frog liver to override the normally tight homeostatic controls on glucose concentrations that are displayed by all vertebrates (including wood frogs outside the winter season). Freezing stress also showed negative effects on CdkIs p21 and p27, both of which exhibited increased levels of inhibitory phosphorylation after freezing exposure (Fig. 3.9A). p21 and p27 interact with Cdks to inhibit the necessary Cdks-cyclin complex assembly, thereby suppressing cell cycle progression. The p21 and p27 results may contribute to the observed lesser inhibition of cell cycle components in response to freezing stress. The phosphorylation levels of both CdkIs mostly decreased upon anoxia and dehydration (Figs. 3.9B, 3.9C), consistent with the results observed in the responses of Cdks and cyclins. Upon thawing, it appears that the cell cycle progression might still be suppressed, which agrees with previous findings that a number of complex biological processes take time to recover after wood frogs have thawed (Layne et al., 1998).

The results from Chapter 3 confirmed my hypothesis. In addition, stress-specific patterns were observed in that freezing had a lesser impact on the expression of cell cycle components than did dehydration and anoxia. Furthermore, recovery from the three stress conditions also exhibited stress-specific responses of the cell cycle components. Taken 
together, these findings suggest that freezing and thawing induces unique cell cycle regulation patterns in wood frog liver.

\section{Liver Akt activation and cell preservation}

The stress-specific response of cell cycle factors in liver suggests that liver might be more active under freezing stress than anoxia or dehydration. The unique pattern of liver cyclin D1 could be in favour of glucose accumulation. As mentioned above, these freezing-specific responses are ultimately controlled by stress-responsive signal transduction. Therefore, based on the observations from Chapter 3, the next step of the thesis was to explore a potential signaling pathway that plays crucial roles in the freeze tolerance by wood frog (Chapter 4). As Chapter 3 demonstrated the phosphorylation state of p21 (Thr-145) increased significantly during freezing but dropped considerably after anoxia and dehydration (Fig. 3.9). The upstream kinase for this phosphorylation event on p21 is Akt, which is well known to mediate signals that promote cell proliferation (Zhou et al., 2001). Therefore, the results for $\mathrm{p} 21$ phosphorylation status led to the hypothesis that Akt signaling was activated in liver under freezing exposure but not during anoxia or dehydration.

Activation of Akt is dependent on its phosphorylation at Ser473 and Thr308 (Zhang et al., 2011). Therefore, to test the hypothesis, Chapter 4 assessed the protein expression level and phosphorylation status of Akt in the liver of wood frogs under freezing, anoxia and dehydration stresses. The response of Akt to freeze-thaw was also examined in other tissues (muscle, heart and kidney). The results showed elevated phosphorylation of both Akt sites in liver during freezing exposure, whereas the opposite 
occurred under anoxia or dehydration stresses (Figs. 4.2, 4.3). These results strongly indicated a freeze-specific activation of Akt in liver (and inhibition under anoxia and dehydration). In addition, data from other tissues (muscle, heart and kidney) also showed signs of inhibition of the Akt signaling, which agrees with the general pattern of prioritizing energy use under stress conditions that is seen in many systems. The Akt pathway mainly promotes ATP-expensive events such as cell proliferation and protein synthesis that should be interrupted in cells under energy stress. Hence, the results suggested that inhibition of Akt may be a critical step to metabolic rate depression in non-hepatic organs during the course of freezing exposure.

In addition to promoting cell cycle progression, an active Akt pathway also plays key roles in cell preservation. One well-known mechanism underlying Akt-dependent cell preservation is its anti-apoptosis action. Multiple downstream targets of the Akt pathway are involved in this process. For one, Akt-mediated p21 phosphorylation is directly related to anti-apoptosis and pro-survival. As discussed in Chapter 3, phosphorylated p21 (Thr145) is sequestered in the cytoplasm by 14-3-3 proteins, thereby preventing it from interacting with Cdks and subsequently suppressing cell cycle progression. Multiple studies have reported that cytoplasmic $\mathrm{p} 21$ serves as an apoptosis inhibitor (Suzuki et al., 1999; Asada et al., 1999; Zhou et al., 2001; Gartel and Tyner, 2002). Cytoplasmic p21 forms a complex with ASK1 (apoptosis-signal-regulating kinase 1) to inhibit p38 (MAPK)-dependent apoptosis (Asada et al., 1999). Therefore, the observed increased in the phosphorylation state of p21 in the liver of frozen frogs may also mediate an Akt/p21-dependent anti-apoptotic effect. Active Akt is also reportedly able to relocate to the nucleus to phosphorylate its nuclear targets, such as transcription 
factors, some of which are also involved in cell preservation pathways, such as FOXO1

(Zhang et al., 2011). FOXO1 is a transcription factor that promotes the expression of the death signal FasL (Brunet et al., 1999). Akt phosphorylation of FOXO1 causes its nuclear export to the cytoplasm and subsequent ubiquitin-dependent degradation (Zhao et al., 2004; Huang et al., 2005). The data in Chapter 4 demonstrated the localization of active Akt to the nucleus in liver in response to freezing (Fig. 4.4), suggesting that the freezing responsive activation of Akt may also target nuclear proteins. The data showed that Akt-dependent phosphorylation of FOXO1 at Ser-256 increased significantly in the liver of frozen frogs as compared with control animals (Fig. 4.8). Hence, combining the observations of enhanced phosphorylation of p21 (Chapter 3) and FOXO1 (Chapter 4) during freezing, correlated with the activation of Akt in liver, the conclusion can be made that Akt contributes to cell preservation strategies in the liver of wood frogs experiencing whole body freezing. This may be critical to maintaining liver function as the source of glucose cryoprotectant production and the site of freeze-specific synthesis of novel protective proteins (Biggar et al., 2013) during freezing exposure.

\section{Mechanisms behind liver Akt activation}

Given the data reported above, it was important to next analyze the molecular mechanisms of the freeze-specific activation of Akt in liver. Activation of Akt requires the protein to be phosphorylated on Ser473 and Thr308. The function of these phosphorylation events differs from each other (Zhang et al., 2011). As described in Chapter 4, Ser473 phosphorylation introduces a conformational change in the Akt protein, creating easy access for the second kinase to phosphorylate Thr308 which 
activates the kinase function. Hence, Ser473 phosphorylation on Ser473 is believed to provide more of a regulatory role, whereas phosphorylation on Thr308 is crucial to Akt activation. PDK1-mediated Thr308 phosphorylation also requires the conversion of PI $[3,4] \mathrm{P} 2$ to PIP3, catalyzed by PI3K. The main inhibitor of Akt activation is the phosphatase PTEN, which functions oppositely to PI3K (Oudit and Penninger, 2009). Therefore, to explore the mechanisms behind the Akt liver action in wood frog during freezing, it was crucial to investigate the regulation of PDK1 and PTEN.

PDK1 is subject to autophosphorylation regulation at Ser241, which activates the kinase action of the protein (Casamayor et al., 1999). Thus, Chapter 4 assessed the protein expression level and phosphorylation status of PDK1, as well as gene expression patterns of PTEN in wood frog liver. The results revealed that while the relative protein level of PDK1 remained at a control level, the relative amount of phospho-PDK1 (Ser241) increased considerably during freezing, suggesting an elevated presence of active PDK1 under stress exposure (Fig. 4.5). By contrast, PTEN relative protein levels dropped significantly in liver upon during freezing (Fig. 4.6A). These data suggest that both PDK1 and PTEN contribute to Akt activation in the liver of frozen frogs. Interestingly, transcript levels of PTEN unexpectedly increased in liver upon freezing exposure (Fig. 4.6A). This indicated the probable involvement of post-transcriptional regulation in controlling the disjoint between elevated PTEN transcripts versus decreased PTEN protein in liver of frozen frogs. Indeed, the subsequent analysis of microRNAs showed that all three PTEN-related miRNAs tested increased significantly throughout the freeze-thaw cycle (Fig. 4.7), confirming the existence of a powerful potential posttranscriptional control mechanism available to regulate PTEN under freezing conditions. 
Protein synthesis and Akt signaling

Activation of Akt may affect downstream other pathways in addition to cell cycle and anti-apoptosis. TORC1-dependent mRNA translation (protein synthesis) is another key target of Akt. Generally, the Akt pathway promotes TORC1 dependent protein synthesis by inhibitory phosphorylation of TORC1 inhibitors. As described in Chapter 5, TORC1 kinase activity relies on GTP-RHEB. The main inhibitor complex TSC1/TSC2 converts GTP-RHEB to the inactive GDP-RHEB, subsequently preventing TORC1 from being activated (Castro et al., 2003). Active Akt phosphorylates TSC2, thereby inhibiting its interaction with TSC1 and ultimately reliving the inhibitory effect of TSC1/TSC1 on TORC1 (Cai et al., 2006). Another TORC1 inhibitor is PRAS40, which is also a direct substrate of Akt. PRAS40 inhibits TORC1 by interacting with the kinase domain of TOR in response to environmental stresses such as nutrient deprivation (Vander Haar et al., 2007). Active Akt phosphorylates PRAS40 at Thr-246, which is then bound by a 14-3-3 protein and dissociates from the TOR:PRAS40 interaction (Vander Haar et al., 2007; Wang et al., 2007). Therefore, the phosphorylation status of TSC2 and PRAS40 are crucial parameters to assess to determine the role of Akt in the activation on TORC1-dependent protein synthesis.

The data in Chapter 5 suggested that the Akt-dependent phosphorylation level of TSC2 (Ser-939) increased significantly in the liver of frozen frogs, which is consistent with the activation of Akt pathway (Fig. 5.7A). The results also indicated that the phospho-TSC2 level dropped in most other tissues in response to freezing (Fig. 5.7A), agreeing with the demonstrated inhibition of Akt activation observed in those tissues 
(Chapter 4). However, the phosphorylation level of PRAS40 failed to respond to freezing exposure, as observed in Chapter 5 (Fig. 5.7B). This differential response by TSC2 and PRAS40 revealed, for the first time, that activation of Akt does not necessarily mean that universal and unified responses by downstream targets occur. In fact, the PRS40 pattern probably reflects the hypometabolic condition in wood frogs during freezing exposure, given that the protein is sensitive to nutrient availability (or, more precisely, the imbalance between energy production and expenditure). The unaltered phosphorylation level of PRAS40 observed in liver and all other tissues tested after $24 \mathrm{~h}$ freezing may indicate that a new equilibrium between ATP output and consumption has been established for freezing survival thanks to the repressed metabolic rate. Furthermore, signs of Akt-dependent TORC1 activation in liver seem to be contrary to the general energy preservation strategy discussed to a great extent in the thesis. With the possibility that Akt regulated its downstream targets differentially according to environmental and/or metabolic conditions, and also in the hope of assessing the actual response of TORC1dependent protein synthesis to freezing stress, an investigation on the TORC1 complex itself appeared to be inevitable (Chapter 5).

\section{Protein synthesis and TORC1 regulation}

One of the key steps of TORC1-dependent protein synthesis is assembly of the TORC1 complex. As mentioned in Chapter 5, the complex contains TOR that provides the kinase activity, and Raptor and G $\beta \mathrm{L}$, both of which play regulatory roles in maintaining the structure of the complex (Hay and Sonenberg, 2004). Both TOR and Raptor are regulated by phosphorylation. Studies have shown that Ser-2448 
phosphorylated TOR is the form found in the TORC1 complex (Copp et al., 2009). Ser863 phosphorylation of Raptor enhances the kinase activity of the complex. Chapter 5 examined the TORC1 pathway by first assessing protein expression levels as well as the phosphorylation status of TOR and Raptor in liver and other tissues (muscle, heart and kidney). While a tissue-specific pattern did occur, generally the protein expression level of TOR decreased in response to freezing and recovered upon thawing, indicating an inhibitory regulation on the complex in frozen frogs. Among all tissues tested, liver showed a lesser inhibitory effect on protein expression and phosphorylation levels during freezing since only the TOR levels dropped while the others remained unchanged compared to control values (Figs. 5.2-5.4). This is consistent with the observation that the Akt pathway was activated in liver but inhibited in other tissues after $24 \mathrm{~h}$ freezing. These findings are also consistent with the fact that liver is known to synthesize and export into the blood at least one freeze-specific novel protein, FR10, as the frogs freeze (while also synthesizing two other novel proteins, Li16 and FR47 that remain within hepatocytes) (Storey and Storey, 2004a; Biggar et al. 2013).

The TORC1 complex was examined using CO-IP to future explore the response of TORC1 to freeze-thaw exposure, since ultimately the function of TORC1 is dependent on complex assembly. As shown in Chapter 5, freezing exposure led to a universal decrease in P-TOR:Raptor interaction in all four tissues tested, strongly suggesting suppression of TORC1 complex assembly (Fig. 5.8). However, liver still exhibited a lesser inhibition of the interaction with a presence of P-TOR (Ser-2448) signal remaining in frozen animals (Fig. 5.8). Upon thawing, it appeared that the interaction was recovered (Fig. 5.8). Protein expression and TORC1-dependent phosphorylation levels of 4EBP and 
S6K were also assessed as downstream targets of TORC1. The phosphorylation level of 4EBP dropped substantially in all four tissues after $24 \mathrm{~h}$ freezing exposure and recovered upon $8 \mathrm{~h}$ thaw (Fig. 5.6A). Similarly, the level of phospho-S6K dropped in most tissues and was restored after thawing (Fig. 5.6B). These results are consistent with the observation from the CO-IP experiment and further confirmed that the function of TORC1 was inhibited in all four tissues in response to freezing. Furthermore, since both 4EBP and S6K are deeply involved in mRNA translation initiation, the observed phosphorylation status suggests that translation (protein synthesis) may be inhibited in all tissues upon freezing and recovered after $8 \mathrm{~h}$ thawing, which fits with a global energy preservation strategy in the frozen state.

Therefore, the data suggest that the activation of the Akt pathway in liver did not promote a general protein synthesis response during freezing but may instead support the synthesis of a selected suite of freeze-responsive proteins, including the three novel proteins previously shown to be produced in wood frog liver during freezing. The data from Chapter 5 once again indicated a differential regulation pattern of Akt downstream targets in liver in response to freezing. However, a lesser inhibition effect on TORC1 assembly in the liver of frozen frogs was observed, which possibly resulted from the activation of Akt in the tissue. This also suggests a higher protein synthesis activity in liver than in other tissues during freezing.

\section{Nrf2-dependent antioxidant defense}

A unique adaptation in wood frog freezing survival is the use of glucose as a cryoprotectant. In fact, all previous Chapters demonstrated that the liver (the source of 
the glucose synthesis) was more active that any other tissue in response to freezing stress. As mentioned in Chapter 1, the cyroprotectant protects cells from osmotic stress and extensive cell shrinkage during freezing due to the colligative action of high glucose. However, high glucose content may elevate ROS production and introduce oxidative stress. In addition, wood frogs also face oxidative stress associated with ischemiareperfusion during freeze-thaw. Therefore, as described in Chapter 1, antioxidant defense strategies are also critical for freeze tolerance. Chapter 6 evaluated antioxidant defenses to freeze-thaw in wood frog organs and also analyzed the antioxidant responses to high glucose-injection into control frogs as well as novel regulation of the Nrf2 transcription factor by glucose.

Data from a multiplex analysis of the protein expression levels of antioxidant enzymes in muscle and liver of wood frogs showed that most were not affected by freezethaw exposure (Figs. 6.1A, 6.2A). This fit with findings from a previous study of antioxidant enzyme activities (Joanisse and Storey, 1996) and suggests that regulation of antioxidant enzymes is likely more focused on enzyme activity and post-translational modifications than on protein expression levels. This fits with a general energy conservation strategy in wood frogs during hypometabolism. Interestingly, it appears that high plasma glucose content ( $200 \mathrm{mM}$, via glucose injection) also affects the expression levels of several antioxidant enzymes in liver, suggesting a role of glucose in antioxidant defense (Figs. 6.1B). In fact, the assays of antioxidant capacity in glucose-injected frogs suggested that high glucose content stimulates antioxidant capacity in muscle (Fig. 6.3). This suggests that hyperglycemic conditions are able to influence antioxidant adaptations in the wood frog, independent of freezing temperatures. 
Preliminary studies demonstrated the responses during freezing of enzymes that are known to be under Nrf2 transcription factor control (Joanisse and Storey, 1996). This lead me to further explore the role of this transcription factor in freeze-responsive antioxidant defense in wood frogs, and the effects of glucose on Nrf2-dependent antioxidant defense (Chapter 6). In general, muscle exhibited similar Nrf2 responses under freezing exposure as those under high glucose content; whereas liver exhibited condition-specific regulation. The data showed that while the protein expression levels of Nrf2 failed to exhibit appreciable changes upon freezing exposure, the DNA binding activity of Nrf2 was enhanced significantly in both liver and muscle (6.4A, 6.7B). The data from the muscle multiplex analysis revealed increased protein levels of the Nrf2 downstream target PRX2, suggesting activation of Nrf2 function in the tissue in response to freezing exposure. Transcript levels of the Nrf2 downstream, gstal, also increased in liver upon freezing exposure, which confirmed the activation of Nrf2 in liver (Fig. 6.8A).

The in vitro DNA binding assay showed that $200 \mathrm{mM}$ glucose was able to stimulate Nrf2-DNA binding in both liver and muscle as compared with the no glucose control (Fig. 6.7C). This suggests that glucose (200 mM) is capable of enhancing Nrf2 DNA binding activity in vivo in wood frogs, since binding of Nrf2 from both tissues exhibited similar responses to the presence of high glucose content. However, gstal transcript levels failed to show a consistent increase. Given that gstal transcript levels increased in liver of frozen frogs, it seems that subzero freezing temperatures are necessary for the glucose-related expression of gstal. As discussed in Chapter 6, this might be related to the GLUT-dependent glucose distribution, since previous studies 
showed a higher GLUT content in autumn frogs than summer animals (Pessin and Bell, 1992; King et al., 1995).

It also appears that, in liver, the response of Nrf2 to glucose was different with and without other components of freezing stress (i.e. low temperature, anoxia and dehydration), as suggested by the data from frozen and glucose-loaded frogs. The results suggested that whereas the protein level of Nrf2 increased upon glucose injection, all antioxidant-related downstream targets failed to show consistent responses (Figs. 6.1B, 6.8B). Given the newly discovered roles of Nrf2 in promoting glycolysis and the pentose phosphate pathway (Mistsuishi et al., 2012), it is possible that the increase of Nrf2 expression upon glucose injection was dedicated to promote NADPH production, which is essential to the function of the antioxidant enzymes including glutathione reductase and thioredoxin reductase (Riganti et al., 2012).

Akt-dependent Nrf2 regulation (GSK3)

In fact, the Akt pathway is also able to regulate Nrf2 through phosphorylation of GSK3 $\beta$. GSK3 $\beta$ is one of the upstream inhibitors of Nrf2. The GSK3 $\beta$-dependent phosphorylation of Nrf2 promotes its cytoplasmic sequestration, which leads to inhibition of its function (Salazar et al., 2006). Akt controls GSK3 $\beta$ via phosphorylation as well. Active Akt phosphorylates GSK3 $\beta$ to stimulate its degradation (Jain and Jaisweal, 2007). Chapter 6 assessed the Akt-dependent phosphorylation state of GSK3 3 . The data showed that the level of phosphorylated GSK3 $\beta$ increased significantly in liver but decreased in muscle in response to freezing (Fig. 6.6A). These data matched the patterns of Akt activation in liver and inhibition in muscle as demonstrated in Chapter 4. 
Therefore, liver activation of Akt might also contribute to antioxidant defense in wood frogs when facing whole body freezing.

\section{Glucose and gene expression}

Chapter 6 suggested, for the first time in wood frogs, that high glucose content is able to influence gene expression and the posttranslational regulation of protein transcripts. First of all, glucose injection $(\sim 200 \mathrm{mM})$ exhibited its effect on the protein expression level of Nrf2 and GSK3 $\beta$ (Figs. 6.4B, 6.5B). Secondly, the data showed that the phosphorylation state of GSK3 $\beta$ was also affected by glucose injection (Fig. 6.6B). Furthermore, the in vitro studies showed that glucose could influence transcription factor DNA binding activity. Not surprisingly, the data also suggest that glucose injection managed to regulate transcript levels of gstal (Fig. 6.8B). In fact, previous studies have identified a GRE (glucose response element) as well as glucose-responsive transcription factors that are able to regulate gene expression in liver via the ChRE (carbohydrate response element) present in the promoter region of many genes in various model species; these typically allow modified gene/protein expression in response to dietary glucose availability (Sander $\boldsymbol{e t}$ al., 1998; Yamashita et al., 2001). Given the importance of glucose as a cryoprotectant in wood frog, it is not surprising that genes involved in major cellular processes are also subject to regulation by glucose. 


\section{Conclusions}

Glucose accumulation plays a crucial role in wood frog freezing survival as the cryoprotectant employed by the animal. As described in the Chapter1, liver is the source for glucose production and facilitates its distribution via the presence of the bidirectional GLUT2 transporter in liver plasma membranes. Findings from Chapter 3 suggest that cell cycle progression is less inhibited in liver under freezing exposure than during other stresses (anoxia and dehydration). Also, although still inhibited, the TORC1 pathway was more active in liver than other tissues upon freezing exposure (Chapter 5). The data from all chapters support the concept of energy conservation strategies under stress conditions where the ATP-consuming events need to be suppressed and reprioritized during hypometabolism. However, my thesis also suggested that both the cell cycle and the TORC1 pathway for regulating protein synthesis were less inhibited in liver under freezethaw conditions than in response to other stresses or in other organs. More importantly, the Akt pathway was activated specifically in liver by freezing stress (Chapter 4). As discussed above, a main well-known action of the Akt pathway is to promote cell survival and energy expensive cellular events. This suggests that liver was indeed more active than other tissues under freezing exposure under the regulation of the Akt pathway. The liver and Akt responses to freezing stress were in favour of glucose production and distribution. Furthermore, it seems that the pathway may be involved in activating antiapoptotic events in the liver of frozen frogs. These all suggest that wood frog liver is more active under freezing stress and Akt signaling seems to play pivotal role in stress responsive signal transduction in wood frog. 
The hyperglycemic environment during freezing and thawing periods can lead to increased risk of oxidative stress. My thesis demonstrated that high glucose content is able to affect the antioxidant defense status in muscle and liver of wood frogs. More importantly, the Nrf2 transcription factor was shown to be glucose-responsive through both in vivo and in vitro approaches. Furthermore, glucose injection was able to regulate the expression of selected genes and the posttranslational modification of selected protein products in wood frogs. These all suggest that glucose offers not only colligative cryoprotection to structurally protect frog cells during freezing but also has cytoprotective actions that help to preserve cellular metabolism. Furthermore, activation of the Akt pathway in liver may be also involved in the stimulating Nrf2-dependent antioxidant defense through GSK3 $\beta$.

In conclusion, my thesis addressed and confirmed the overall hypothesis by thoroughly assessing the Akt pathway and related downstream targets (cell cycle, TOR1dependent protein synthesis, and Nrf2-dependent antioxidant defense) in multiple tissues and under multiple stresses using a variety of techniques. In addition, the thesis demonstrated that Akt activation produced different responses by downstream targets under different environmental and metabolic contexts, while also implicating additional metabolic actions by high glucose in frozen wood frogs. 


\section{REFERENCES}


Alam J, Stewart D, Touchard C, Boinapally S, Choi AMK, Cook JL. 1999. Nrf2, a cap' $n$ 'collar transcription factor, regulates induction of the heme oxygenase-1 gene. J. Biol. Chem. 274: 26071 - 26078.

Alessi DR, James SR, Downes CP, Holmes AB, Gaffney PR, Reese CB, Cohen P. 1997. Characterization of a 3-phosphoinositide-dependent protein kinase which phosphorylates and activates protein kinase B alpha. Curr Biol. 7: 261 - 269.

Allan M, Storey KB. 2012. Expression of NF- $\mathrm{kB}$ and downstream antioxidant genes in skeletal muscle of hibernating ground squirrels, Spermophilus tridecemlineatus. Cell Biochem. Funct. 30: $166-174$.

Ali A, Hoeflich KP, Woodgett JR. 2001. Glycogen synthase kinase-3: properties, functions and regulation. Chem. Rev. 101: $2527-2540$.

Andjelkovic M, Alessi DR, Meier R, Fernandez A, Lamb NJC, Frech M, Cron P, Cohen P, Lucocq JM, Hemmings BA. 1997. Role of translocation in the activation and function of protein kinase B. J. Biol. Chem. 272: 31515 - 31524.

Asada M, Yamada T, Ichijo H, Delia D, Miyazono K, Fukumuro K, Mizutani S. 1999. Apoptosis inhibitory activity of cytoplasmic p21 (Cip1/WAF1) in monocytic differentiation. EMBO. J. 18:1223 - 1234.

Bartel DP. 2009. MicroRNAs: target recognition and regulatory functions. Cell 136: 215 -233 .

B rtov I, Otyepka M, Kr z , Koca . 2004. Activation and inhibition of cyclindependent kinase-2 by phosphorylation; a molecular dynamics study reveals the functional importance of the glycine-rich loop. Protein Sci. 13: 1449 - 1457.

Bellacosa A, Testa JR, Staal SP, Tsichlis PN. 1991. A retroviral oncogene, akt, encoding a serine-threonine kinase containing an SH2-like region. Science 254: $274-277$.

Berman DI, Leirikh AN, Mikhailova EI. 1984. Winter hibernation of the Siberian salamander Hynobius keyserlingi. J. Evol. Biochem. Physiol. 3: 323 - 327. (in Russian with English summary)

Berry LD, Gould KL. 1996. Regulation of Cdc2 activity by phosphorylation at T14/Y15. Prog. Cell Cycle Res. 2: 99 - 105.

Bienvenu F, Jirawatnotai S, Elias JE, Meyer CA, Mizeracka K, Marson A, Frampton GM, Cole MF, Odom DT, Odajima J, Geng Y, Zagozdzon A, Jecrois M, Young RA, Liu XS, Cepko CL, Gygi SP, Sicinski P. 2010. Transcriptional role of cyclin D1 in development revealed by a genetic-proteomic screen. Nature 463: $374-$ 378.

Biggar KK, Dubuc A, Storey K. 2009. MicroRNA regulation below zero: differential expression of miRNA-21 and miRNA-16 during freezing in wood frogs. Cryobiology.59: $317-321$.

Biggar KK, Kornfeld SF, Storey KB. 2011. Amplification and sequencing of mature microRNAs in uncharacterized animal models using stem-loop reverse transcription-polymerase chain reaction. Anal. Biochem. 416: 231 - 233. 
Biggar KK, Storey KB. 2009. Perspectives in cell cycle regulation: lessons from an anoxic vertebrate. Curr. Genomics 10: 573-584.

Bilodeau JF, Chatterjee S, Sirard MA, Gagnon C. 2000. Levels of antioxidant defenses are decreased in bovine spermatozoa after a cycle of freezing and thawing. Mol. Reprod. Dev. 55: 282 - 288.

Booher RN, Holman PS, Fattaey A. 1997. Human Myt1 is a cell cycle-regulated kinase that inhibits Cdc2 but not Cdk2 activity. J. Biol. Chem. 272: 22300 - 22306.

Brunet A, Bonni A, Zigmond MJ, Lin MZ, Juo P, Hu LS, Anderson MJ, Arden KC, Blenis J, Greenberg ME. 1999. Akt promotes cell survival by phosphorylating and inhibiting a forkhead transcription factor. Cell 96: $857-868$.

Bulavin DV, Amundson SA, Fornace AJ. 2002. p38 and Chk1 kinases: different conductors for the G(2)/M checkpoint symphony. Curr. Opin. Genet. Dev.12: 92 -97 .

Busino L, Donzelli M, Chiesa M, Guardavaccaro D, Ganoth D, Dorrello NV, Hershko A, Pagano M, Draetta GF. 2003. Degradation of Cdc25A by beta-TrCP during S phase and in response to DNA damage. Nature 426: 87 - 91.

Campos R, Maureira F, Garrido A, Valenzuela A. 1993. Different glutathione redox status and lipid peroxidation in the cortex and the medulla of the rat kidney subjected to ischemia-reperfusion stress. Comp. Biochem. Physiol. B. 105: 157 163.

Cai SL, Tee AR, Short JD, Bergeron JM, Kim J, Shen J, Guo R, Johnson CL, Kiguchi K, Walker CL. 2006. Activity of TSC2 is inhibited by AKT-mediated phosphorylation and membrane partitioning. J. Cell. Biol. 173: 279 - 289.

Campos R, Maureira F, Garrido A, Valenzuela A. 1993. Different glutathione redox status and lipid peroxidation in the cortex and the medulla of the rat kidney subjected to ischemia-reperfusion stress. Comp. Biochem. Physiol. B. 105: 157 163.

Casamayor A, Morrice NA, Alessi DR. 1999. Phosphorylation of Ser-241 is essential for the activity of 3-phosphoinositide-dependent protein kinase-1: identification of five sites of phosphorylation in vivo. Biochem. J. 342: $287-292$.

Castro, A.F., Rebhun, J.F., Clark, G.J., and Quilliam, L.A. 2003. Rheb binds tuberous sclerosis complex 2 (TSC2) and pro- motes $\mathrm{S} 6$ kinase activation in a rapamycinand farnesylation-dependent manner. J. Biol. Chem. 278: 32493 - 32496.

Carey HV, Andrews MT, Martin SL. 2003. Mammalian hibernation: cellular and molecular responses to depressed metabolism and low temperature. Physiol. Rev. 83: $1153-1181$.

Chanas SA, Jiang Q, McMahon M, McWalter GK, McLellan LI, Elcombe CR, Henderson CJ, Wolf CR, Moffat GJ, Itoh K, Yamamoto M, Hayes JD. 2002. Loss of the Nrf2 transcription factor causes a marked reduction in constitutive and inducible expression of the glutathione S-transferase Gsta1, Gsta2, Gstm1, 
Gstm2, Gstm3 and Gstm4 genes in the livers of male and female mice. Biochem. J. 365: $405-416$.

Cheng JQ, Lindsley CW, Cheng GZ, Yang H, Nicosia SV. 2005. The Akt/PKB pathway: molecular target for cancer drug discovery. Oncogene 24: 7482 - 7492.

Cheng M, Olivier P, Diehl JA, Fero M, Roussel MF, Roberts JM, Sherr CJ. 1999. The p21(Cip1) and p27(Kip1) Cdk 'inhibitors' are essential activators of cyclin Ddependent kinases in murine fibroblasts. EMBO. J. 18: 1571 - 1583.

Chiang GG, Abraham RT. 2005. Phosphorylation of mammalian target of rapamycin (mTOR) at Ser-2448 is mediated by p70S6 kinase. J. Biol. Chem. 280: 25485 25490.

Churchill TA, Storey KB. 1993. Dehydration tolerance in wood frogs: a new perspective on the development of amphibian freeze tolerance. Am. J. Physiol. 265, R1324R1332.

Churchill TA, Storey KB. 1994. Metabolic responses to dehydration by liver of the wood frog, Rana sylvatica. Can. J. Zool. 72: $1420-1425$.

Claussen DL, Townsley MD, Bausch RG. 1990. Supercooling and freeze tolerance in the European wall lizard, Podarcis muralis. J. Comp. Physiol. B, 160: 137-143.

Coffer PJ, Woodgett JR. 1991. Molecular cloning and characterization of a novel putative protein-serine kinase related to the cAMP-dependent and protein kinase $\mathrm{C}$ families. Eur. J. Biochem. 201: 475-481.

Conlon JM, Yano K, Chartrel N, Vaudry H, Storey KB. 1998. Freeze tolerance in the wood frog Rana sylvatica is associated with unusual structural features in insulin but not in glucagon. J. Mol. Endocrinol. 21: 153 - 159.

Copp J, Manning G, Hunter T. 2009. TORC-specific phosphorylation of mammalian target of rapamycin (mTOR): phospho-Ser2481 is a marker for intact mTOR signaling complex 2. Cancer Res. 69: 1821 - 1827.

Costanzo JP, Lee RE, Lortz PH. 1993. Glucose concentration regulates freeze tolerance in the wood frog Rana sylvatica. J. Exp. Biol. 181: 245-255.

Courteau L, Storey KB, Morin PJr. 2012. Differential expression of microRNA species in a freeze tolerant insect, Eurosta solidaginis. Cryobiology 65: $210-214$.

Cowan KJ, Storey KB. 2001. Freeze-thaw effects on metabolic enzymes in wood frog organs. Cryobiology 43: $32-45$.

Cowan KJ, Storey KB. 2003. Mitogen-activated protein kinases: new signaling pathways functioning in cellular responses to environmental stress. J. Exp. Biol. 206: 11071115.

De Schutter K, oub s , Cools T, e rkest A, Corellou F, Babiychuk E, an Der Schueren E, Beeckman T, Kushnir S, Inzé D, De Veylder L. 2007. Arabidopsis WEE1 kinase con- trols cell cycle arrest in response to activation of the DNA integrity checkpoint. Plant Cell 19: $211-225$. 
Dieni CA, Bouffard MC, Storey KB. 2012. Glycogen synthase kinase-3: cryoprotection and glycogen metabolism in the freeze-tolerant wood frog. J Exp Biol. 215: $543-$ 551.

Dinkova-Kostova AT, Holtzclaw WD, Cole RN, Itoh K, Wakabayashi N, Katoh Y, Yamamoto M, Talalay P. 2002. Direct evidence that sulfhydryl groups of Keap1 are the sensors regulating induction of phase 2 enzymes that protect against carcinogens and oxidants. Proc. Natl. Acad. Sci. USA 99: 11908 - 11913.

Downey JM. 1990. Free radicals and their involvement during long-term myocardial ischemia and reperfusion. Annu. Rev. Physiol. 52: 487-504.

Draetta G, Luca F, Westendorf J, Brizuela L, Ruderman J, Beach D. 1989. Cdc2 protein kinase is complexed with both cyclin A and B: evidence for proteolytic inactivation of MPF. Cell 56: $829-838$.

Ebert MS, Sharp PA. 2012. Roles for microRNAs in conferring robustness to biological processes. Cell 149: 515-524.

Elstrom RL, Bauer DE, Buzzai M, Karnauskas R, Harris MH, Plas DR, Zhuang H, Cinalli RM, Alavi A, Rudin CM, Thompson CB. 2004. Akt stimulates aerobic glycolysis in cancer cells. Cancer Res. 64: $3892-3899$.

Embi N, Rylatt DB, Cohen P. 1980. Glycogen synthase kinase-3 from rabbit skeletal muscle. Separation from cyclic-AMP-dependent protein kinase and phosphorylase kinase. Eur. J. Biochem. 107: 519 - 527.

English TE, Storey KB. 2003. Freezing and anoxia stresses induce expression of metallothionein in the foot muscle and hepatopancreas of the marine gastropod, Littorina littorea. J. Exp. Biol. 206: 2517 - 2524.

Feder ME, Hofmann GE. 1999. Heat-shock proteins, molecular chaperones, and the stress response: evolutionary and ecological physiology. Annu. Rev. Physiol. 6: 243-282.

Fish JE, Santoro MM, Morton SU, Yu S, Yeh RF, Wythe JD, Ivey KN, Bruneau BG, Stainier DY, Srivastava D. 2008. miR-126 regulates angiogenic signaling and vascular integrity. Dev. Cell. 15; $272-284$.

Foster KG, Acosta-Jaquez HA, Romeo Y, Ekim B, Soliman GA, Carriere A, Roux PP, Ballif BA, Fingar DC. 2010. Regulation of mTOR complex 1 (mTORC1) by raptor Ser863 and multisite phosphorylation. J. Biol. Chem. 285: 80 - 94.

Fuller BJ, Gower JD, Green CJ. 1988. Free radical damage and organ preservation: fact or fiction? A review of the interrelationship between oxidative stress and physiological ion disbalance. Cryobiology. 25: 377 - 393.

Gartel AL, Tyner AL. 2002. The role of the cyclin-dependent kinase inhibitor p21 in apoptosis. Mol. Cancer Ther. 1: $639-649$.

Gething MJ, Sambrook J. 1992. Protein folding in the cell. Nature 355: 33-45. 
Gingras AC, Raught B, Sonenberg N. 1999. eIF4 initiation factors: effectors of mRNA recruitment to ribosomes and regulators of translation. Annu. Rev. Biochem. 68: $913-963$.

Grimes CA, Jope RS. 2001. The multifaceted roles of glycogen synthase kinase 3 beta in cellular signaling. Prog. Neurobiol. 65: $391-426$.

Gwinn DM, Shackelford DB, Egan DF, Mihaylova MM, Mery A, Vasquez DS, Turk BE, Shaw RJ. 2008. AMPK phosphorylation of raptor mediates a metabolic checkpoint. Mol. Cell. 30: 214 - 226.

Harashima H, Dissmeyer N, Schnittger A. 2013. Cell cycle control across the eukaryotic kingdom. Trends Cell Biol. 2013 Apr 5. pii: S0962-8924(13)00046-9.

Harper JW, Adami GR, Wei N, Keyomarsi K, Elledge SJ. 1993. The p21 Cdk-interacting protein Cip1 is a potent inhibitor of G1 cyclin-dependent kinases. Cell 75: 805 816.

Harris ED. 1992. Regulation of antioxidant enzymes. FASEB. J. 6: 2675 - 2683.

Hart JR, Vogt PK. 2011. Phosphorylation of AKT: a mutational analysis. Oncotarget 2: $467-476$.

Harvey CJ, Thimmulappa RK, Singh A, Blake DJ, Ling G, Wakabayashi N, Fujii J, Myers A, Biswal S. 2009. Nrf2-regulated glutathione recycling independent of biosynthesis is critical for cell survival during oxidative stress. Free Radic. Biol. Med. 46: $443-453$.

Hay N, Sonenberg N. 2004. Upstream and downstream of mTOR. Genes Dev. 18: 1926 1945.

Hayes JD, Flanagan JU, Jowsey IR. 2005. Glutathione transferases. Annu. Rev. Pharmacol. Toxicol. 45: $51-88$.

Hershey, J.W.B. and Merrick, W.C. 2000. Pathway and mechanism of initiation of protein synthesis. In Translational control of gene expression (ed. M.B. Mathews), pp. 33-88. Cold Spring Harbor Laboratory Press, Cold Spring Harbor, N.Y.

Hirotsu Y, Katsuoka F, Funayama R, Nagashima T, Nishida Y, Nakayama K, Engel JD, Yamamoto M. 2012. Nrf2-MafG heterodimers contribute globally to antioxidant and metabolic networks. 2012. Nucleic Acids Res. 40: 10228 -10239.

Holden CP, Storey KB. 1996. Signal transduction, second messenger, and protein kinase responses during freezing exposures in the wood frog. Am. J. Physiol. 271: R1205 $-\mathrm{R} 1211$.

Holden CP, Storey KB. 1997. Second messenger and cAMP-dependent protein kinase responses to dehydration and anoxia stresses in frogs. J. Comp. Physiol. B. 167: 305-312.

Holden CP, Storey KB. 2000. Purification and characterization of protein kinase A catalytic subunit from liver of the freeze-tolerant wood frog: role in glycogenolysis during freezing. Cryobiology. 40: 323-331. 
Holden HA, Storey KB. 2011. Reversible phosphorylation regulation of NADPH-linked polyol dehydrogenase in the freeze avoiding gall moth, Epiblema scudderiana: role in glycerol metabolism. Arch. Insect Biochem. Physiol. 77: 32-44.

Horie T, Ono K, Nagao K., Nishi H, Kinoshita M, Kawamura T, Wada H, Shimatsu A, Kita T, Hasegawa K. 2008). Oxidative stress induces GLUT4 translocation by activation of PI3-K/Akt and dual AMPK kinase in cardiac myocytes. J. Cell. Physiol. 215: 733 - 742 .

Hresko RC, Mueckler M. 2005. mTOR.RICTOR is the Ser473 kinase for Akt/protein kinase B in 3T3-L1 adipocytes. J. Biol. Chem. 280: 40406 - 40416.

Huang BX, Akbar M, Kevala K, Kim HY. 2011. Phosphatidylserine is a critical modulator for Akt activation. J. Cell Biol. 192: 979 - 992.

Huang H, Regan KM, Wang F, Wang D, Smith DI, van Deursen JM, Tindall DJ. 2005. Skp2 inhibits FOXO1 in tumor suppression through ubiquitin-mediated degradation. Proc. Natl. Acad. Sci. U.S.A. 102:1649 - 1654.

Huang HC, Nguyen T, Pickett CB. 2002. Phosphorylation of Nrf2 at Ser-40 by protein kinase $\mathrm{C}$ regulates antioxidant response element-mediated transcription. J. Biol. Chem. 277: 42769 - 42774.

Huse JT, Brennan C, Hambardzumyan D, Wee B, Pena J, Rouhanifard SH, Sohn-Lee C, le Sage C, Agami R, Tuschl T, Holland EC. 2009. The PTEN-regulating microRNA miR-26a is amplified in high-grade glioma and facilitates gliomagenesis in vivo. Genes Dev. 23: 1327 - 1337.

Iliakis G, Wang Y, Guan J, Wang H. 2003. DNA damage checkpoint control in cells exposed to ionizing radiation. Oncogene 22:5834 - 5847 .

Imadome K, Iwakawa M, Nojiri K, Tamaki T, Sakai M, Nakawatari M, Nakamura E, Tsujii H, Imai T. 2008. Upregulation of stress-response genes with cell cycle arrest induced by carbon ion irradiation in multiple murine tumors models. Cancer Biol. Ther. 7: $208-217$.

Inoki K, Li Y, Zhu T, Wu J, Guan KL. 2002. TSC2 is phosphorylated and inhibited by Akt and suppresses mTOR signalling. Nat. Cell. Biol. 4: 648 - 657.

Isotani, S., Hara, K., Tokunaga, C., Inoue, H., Avruch, J., Yonezawa, K. 1999. Immunopurified mammalian target of rapamycin phosphorylates and activates p70 S6 kinase alpha in vitro. J. Biol. Chem. 274(48): 34493 - 34498.

Jain AK, Jaiswal AK. 2007. GSK-3beta acts upstream of Fyn kinase in regulation of nuclear export and degradation of NF-E2 related factor 2. J. Biol. Chem. 282: $16502-16510$.

Jin J, Shirogane T, Xu L, Nalepa G, Qin J, Elledge SJ, Harper JW. 2003. SCFbeta-TRCP links Chk1 signaling to degrada- tion of the Cdc25A protein phosphatase. Genes. Dev. 17: 3062 - 3074.

Jin IA, Nguyen A, Lim D, Stewart MQ, Elia AE, Yaffe MB. 2005. MAPKAP kinase-2 is a cell cycle check- point kinase that regulates the $\mathrm{G} / \mathrm{M}$ transition and $\mathrm{S}$ phase progression in response to UV irradiation. Mol. Cell 17: $37-48$. 
Jinno S, Suto K, Nagata A, Igarashi M, Kanaoka Y, Nojima H, Okayama H. 1994. $\mathrm{Cdc} 25 \mathrm{~A}$ is a novel phosphatase func- tioning early in the cell cycle. EMBO. J. 13:1549- 1556 .

Joanisse DR, Storey KB. 1994a. Mitochondrial enzymes during overwintering in two species of cold-hardy gall insects. Insect. Biochem. Molec. Biol. 24: 145 - 150.

Joanisse DR, Storey KB. 1994b. Enzyme activity profiles in an overwintering population of freeze-tolerant larvae of the gall fly Eurosta solidaginis. J. Comp. Physiol. B. 164: $247-255$.

Joanisse DR, Storey KB. 1994c. Enzyme activity profiles in an overwintering population of freeze-avoiding gall moth larvae, Epiblema scudderiana. Can. J. Zool. 72(6): 1079-1086.

Joanisse DR, Storey KB. 1996. Oxidative damage and antioxidants in Rana sylvatica, the freeze-tolerant wood frog. Am. J. Physiol. 271: R545 - 553.

Jones PF, Jakubowicz T, Pitossi FJ, Maurer F, Hemmings BA. (1991) Molecular cloning and identification of a serine/threonine protein kinase of the second-messenger subfamily. Proc. Natl. Acad. Sci. U.S.A. 88: 4171-4175.

Kaldis P. 1999. The cdk-activating kinase (CAK): from yeast to mammals. Cell. Mol. Life Sci. 55: $284-296$.

Karlsson-Rosenthal C, Millar JB. 2006. Cdc25: mechanisms of checkpoint inhibition and recovery. Trends Cell Biol. 16: 285 - 292.

Kaspar JW, Niture SK, Jaiswal AK. 2009. Nrf2:INrf2 (Keap1) signaling in oxidative stress. Free Radic. Biol. Med. 47: 1304 - 1309.

Kato M, Putta S, Wang M, Yuan H, Lanting L, Nair I, Gunn A, Nakagawa Y, Shimano H, Todorov I, Rossi JJ, Natarajan R. 2009. TGF-beta activates Akt kinase through a microRNA-dependent amplifying circuit targeting PTEN. Nat. Cell Biol. 11: $881-889$.

Katoh Y, Iida K, Kang MI, Kobayashi A, Mizukami M, Tong KI, McMahon M, Hayes JD, Itoh K, Yamamoto M.. 2005. Evolutionary conserved N-terminal domain of Nrf2 is essential for the Keap1-mediated degradation of the protein by proteasome. Arch. Biochem. Biophys. 433: $342-450$.

Karlsson-Rosenthal C, Millar JB. 2006. Cdc25: mechanisms of checkpoint inhibition and recovery. Trends Cell. Biol. 16: $285-292$.

Kling KB, Costanzo J., Lee RE. 1994. Post-freeze recovery of peripheral nerve function in the freeze-tolerant wood frog, Rana sylvatica. J. Comp. Physiol. B 164: 316320.

Kim DH, Sarbassov DD, Ali SM, Latek RR, Guntur KV, Erdjument-Bromage H, Tempst P, Sabatini DM. 2003. GbetaL, a positive regulator of the rapamycin-sensitive pathway required for the nutrient-sensitive interaction between raptor and mTOR. Mol. Cell. 11: 895 - 904. 
Kim HJ, Vaziri ND. 2010. Contribution of impaired Nrf2-Keap1 pathway to oxidative stress and inflammation in chronic renal failure. Am. J. Physiol. Renal. Physiol. 298: F662 - 671 .

Kimura MT, Yoshida KM, Goto SG. 1998. Accumulation of Hsp70 mRNA under environmental stresses in diapausing and nondiapausing adults of Drosophila triauraria. J. Insect. Physiol. 44: 1009-1015.

King GL, Loeken MR. 2004. Hyperglycemia-induced oxidative stress in diabetic complications. Histochem. Cell. Biol. 122: 333 - 338.

King PA, Rosholt MN, Storey KB. 1993. Adaptations of plasma membrane glucose transport facilitate cryoprotectant distribution in freeze tolerant frogs. Am. J. Physiol. 265: R1036-R1042.

King PA, Rosholt MN, Storey KB. 1995. Seasonal changes in plasma membrane glucose transport in freeze tolerant wood frogs. Can. J. Zool. 73: 1-9.

Kiss AJ, Muir TJ, Lee RE Jr, Costanzo JP. 2011. Seasonal variation in the hepatoproteome of the dehydration and freeze-tolerant wood frog, Rana sylvatica. Int. J. Mol. Sci. 12: 8406-8414.

Kirkman HN, Gaetani GF. 1984. Catalase: a tetrameric enzyme with four tightly bound molecules of NADPH. Proc. Natl. Acad. Sci. USA 81: 4343 - 4347.

Kristal BS, Yu BP. 1992. An emerging hypothesis: synergistic induction of aging by free radicals and Maillard reactions. J. Gerontol. 47: B107 - 114.

Krivoruchko A, Storey KB. 2010a. Molecular mechanisms of turtle anoxia tolerance: A role for NFkappaB. Gene 450: 63 - 69.

Krivoruchko A, Storey KB. 2010b. Activation of antioxidant defenses in response to freezing in freeze tolerant painted turtle hatchlings. Biochim. Biophys. Acta. 1800: $662-668$.

LaBaer J, Garrett MD, Stevenson LF, Slingerland JM, Sandhu C, Chou HS, Fattaey A, Harlow E. 1997. New functional activities for the p21 family of Cdk inhibitors. Genes Dev. 11: 847 - 862.

Layne JR. 1995. Crystallization temperatures of frogs and their individual organs. J. Herpetol. 29: $296-298$.

Layne JR. 1999. Freeze tolerance and cryoprotectant mobilization in the gray treefrog (Hyla versicolor). J. Exp. Zool. 283: 221 - 225.

Layne JR, First MC. 1991. Resumption of physiological functions in the wood frog (Rana sylvatica) after freezing. Am. J. Physiol. 261: R134-R137.

Layne JR, Lee RE, Huang JL. 1990. Inoculation triggers freezing at high subzero temperatures in a freeze-tolerant frog (Rana sylvatica) and insect (Eurosta solidaginis), Can. J. Zool. 68: 506-510.

Layne JR Jr, Costanzo JP, Lee RE Jr. 1998. Freeze duration influences postfreeze survival in the frog Rana sylvatica. J. Exp. Zool. 280: 197 - 201. 
Lee MR, Lee, RE, Strong-Gunderson JM, Minges SR. 1995. Isolation of ice-nucleating active bacteria from the freeze-tolerant frog, Rana sylvatica. Cryobiology. 32: $358-365$.

Lehman N, Ledford B, Di Fulvio M, Frondorf K, McPhail LC, Gomez-Cambronero J. 2007. Phospholipase D2-derived phosphatidic acid binds to and activates ribosomal p70 S6 kinase independently of mTOR. FASEB. J. 21: 1075 - 1087.

Lew DJ, Kornbluth S. 1996. Regulatory roles of cyclin dependent kinase phosphorylation in cell cycle control. Curr. Opin. Cell Biol. 8: 795 - 804.

Li W, Febbraio M, Reddy SP, Yu DY, Yamamoto M, Silverstein RL. 2010. CD36 participates in a signaling pathway that regulates ROS formation in murine VSMCs. J. Clin. Invest. 120: 3996 -4006.

Lolli G, Johnson LN. 2005. CAK-Cyclin-dependent Activating Kinase: a key kinase in cell cycle control and a target for drugs? Cell Cycle 4: $572-577$.

Low FM, Hampton MB, Peskin AV, Winterbourn CC. 2007. Peroxiredoxin 2 functions as a noncatalytic scavenger of low-level hydrogen peroxide in the erythrocyte. Blood 109: 2611 - 2617.

Lyons PJ.,Poitras JJ, Courteau LA, Storey KB, Morin PJr. 2013. Identification of differentially regulated microRNAs in cold-hardy insects. Cryo-Lett. 34: 83-89.

Ma XM, Blenis J. 2009. Molecular mechanisms of mTOR-mediated translational control. Nat. Rev. Mol. Cell. Biol. 10: 307 - 318.

MacDonald JA, Storey KB. 1999. Protein phosphatase responses during freezing and thawing in wood frogs: control of liver cryoprotectant metabolism. Cryo-Lett. 20: 297-306.

Maehama T, Dixon JE. 1998. The tumor suppressor, PTEN/MMAC1, dephosphorylates the lipid second messenger, phosphatidylinositol 3,4,5-trisphosphate. J. Biol. Chem. 273: 13375 - 13378.

Malik AI, Storey KB. 2011. Transcriptional regulation of antioxidant enzymes by FoxO1 under dehydration stress. Gene 485: $114-119$.

Manzella, J.M., Rychlik, W., Rhoads, R.E., Hershey, J.W., and Blackshear, P.J. 1991. Insulin induction of ornithine decarboxylase. Importance of mRNA secondary structure and phosphorylation of eucaryotic initiation factors eIF-4B and eIF-4E. J. Biol. Chem. 266: 2383-2389.

Montagnoli A, Fiore F, Eytan E, Carrano AC, Draetta GF, Hershko A, Pagano M. 1999. Ubiquitination of p27 is regulated by Cdk-dependent phosphorylation and trimeric complex formation. Genes Dev.13: 1181 -1191.

Morin P Jr, Ni Z, McMullen DC, Storey KB. 2008. Expression of Nrf2 and its downstream gene targets in hibernating 13-lined ground squirrels, Spermophilus tridecemlineatus. Mol. Cell. Biochem. 312: 121 - 129. 
Mitsuishi Y, Taguchi K, Kawatani Y, Shibata T, Nukiwa T, Aburatani H, Yamamoto M, Motohashi H. 2012. Nrf2 redirects glucose and glutamine into anabolic pathways in metabolic reprogramming. Cancer Cell. 22: $66-79$.

Muller FL, Lustgarten MS, Jang Y, Richardson A, Van Remmen H. 2007. Trends in oxidative aging theories. Free Radic. Biol. Med. 43: $477-503$.

Muise AM, Storey KB. 1997. Reversible phosphorylation of fructose-1,6-bisphosphatase mediates enzyme role in glycerol metabolism in the freeze avoiding gall moth Epiblema scudderiana. Insect Biochem. Mol. Biol. 27:617-623.

Neijssel OM, Teixeira de Mattos MJ, Tempest DW. 1996. Growth yield and energy distribution, p. 1683-1692. In F. C. Neidhardt, R. Curtiss III, J. L. Ingraham, E. C. C. Lin, K. B. Low, B. Magasanik, W. S. Reznikoff, M. Riley, M. Schaechter, and H. E. Umbarger (ed.), Escherichia coli and Salmonella: cellular and molecular biology, 2nd ed. American Society for Microbiology, Washington, DC.

Ni Z, Storey KB. 2010. Heme oxygenase expression and Nrf2 signaling during hibernation in ground squirrels. Can. J. Physiol. Pharmacol. 88: 379 - 387.

Nielsen MM, Overgaard J, Sørensen JG, Holmstrup M, Justesen J, Loeschcke V. 2005. Role of HSF activation for resistance to heat, cold and high-temperature knockdown. J. Insect Physiol. 51: 1320-1329.

Nioi P, McMahon M, Itoh K, Yamamoto M, Hayes JD. 2003. Identification of a novel Nrf2-regulated antioxidant response element (ARE) in the mouse NAD(P)H:quinone oxidoreductase 1 gene: reassessment of the ARE consensus sequence. Biochem. J. 374: 337 - 348.

Nojima, H., Tokunaga, C., Eguchi, S., Oshiro, N., Hidayat, S.,Yoshino, K., Hara, K., Tanaka, N., Avruch, J., and Yonezawa, K. 2003. The mammalian target of rapamycin (mTOR) partner, raptor, binds the mTOR substrates p70 S6 kinase and 4E-BP1 through their TOR signaling (TOS) motif. J. Biol.Chem. 278: 1546115464.

Oberley LW, Buettner GR. 1979. Role of superoxide dismutase in cancer: a review. Cancer Res. 39: 1141 - 1149 .

Oehler-Jänne C, von Bueren AO, Vuong V, Hollenstein A, Grotzer MA, Pruschy M. 2008. Temperature sensitivity of phospho-Ser(473)-PKB/AKT. Biochem Biophys Res Commun. 375: 399 - 404.

Oudit GY, Penninger JM. 2009. Cardiac regulation by phosphoinositide 3-kinases and PTEN. Cardiovasc. Res. 82: $250-260$.

Pace DA, Manahan DT. 2007. Cost of protein synthesis and energy allocation during development of Antarctic sea urchin embryos and larvae. Biol. Bull. 212: 115 129.

Pajvani UB, Shawber CJ, Samuel VT, Birkenfeld AL, Shulman GI, Kitajewski J, Accili D. 2011. Inhibition of Notch signaling ameliorates insulin resistance in a FoxO1dependent manner. Nat. Med. 17: 961 - 967. 
Park EY, Rho HM. 2002. The transcriptional activation of the human copper/zinc superoxide dismutase gene by 2,3,7,8-tetrachlorodibenzo- $\mathrm{p}$-dioxin through two different regulator sites, the antioxidant responsive element and xenobiotic responsive element. Mol. Cell. Biochem. 240: 47 - 55.

Pearl LH, Barford D. 2002. Regulation of protein kinases in insulin, growth factor and Wnt signalling. Curr. Opin. Struct. Biol. 12: $761-767$.

Peterson RT, Beal PA, Comb MJ, Schreiber SL. 2000. FKBP12-rapamycin-associated protein (FRAP) autophosphorylates at serine 2481 under translationally repressive conditions. J. Biol. Chem. 275: 7416 - 7423.

Perucca P, Cazzalini O, Madine M, Savio M, Laskey RA, Vannini V, Prosperi E, Stivala LA. 2009. Loss of p21 CdkN1A impairs entry to quiescence and activates a DNA damage response in normal fibroblasts induced to quiescence. Cell Cycle 8: $105-$ 114.

Pessin JE, Bell GI. 1992. Mammalian facilitative glucose transporter family: structure and molecular regulation. Annu. Rev. Physiol. 54: 911-930.

Pfister TD , Storey KB. 2006. Responses of protein phosphatases and cAMP-dependent protein kinase in a freeze avoiding insect, Epiblema scudderiana. Arch. Insect Biochem. Physiol. 62, 43-54.

Pietenpol JA, Stewart ZA. 2002. Cell cycle checkpoint signaling: cell cycle arrest versus apoptosis. Toxicology 181: $475-481$.

Qin W, Neal SJ, Robertson RM, Westwood JT, Walker VK. 2005. Cold hardening and transcriptional change in Drosophila melanogaster. Insect Mol. Biol. 14: 607613.

Rada P, Rojo AI, Chowdhry S, McMahon M, Hayes JD, Cuadrado A. 2011. SCF/betaTrCP promotes glycogen synthase kinase 3-dependent degradation of the Nrf2 transcription factor in a Keap1-independent manner. Mol. Cell. Biol. 31: 1121 1133.

Rider MH, Hussain N, Horman S, Dilworth SM, Storey KB. 2006. Stress-induced activation of the AMP-activated protein kinase in the freeze-tolerant frog Rana sylvatica. Cryobiology 53: 297 - 309.

Reeds PJ, Wahle KW, Haggarty P. 1982. Energy costs of protein and fatty acid synthesis. Proc. Nutr. Soc. 41: 155 - 159.

Rieder CL, Cole RW. 2002. Cold-shock and the Mammalian cell cycle. Cell Cycle 1: 169 $-175$.

Rider MH, Hussain N, Dilworth SM, Storey JM, Storey KB. 2011. AMP-activated protein kinase and metabolic regulation in cold-hardy insects. J. Insect Physiol. 57(11):1453 - 1462 .

Roufayel R, Biggar KK, Storey KB. 2011. Regulation of cell cycle components during exposure to anoxia or dehydration stress in the wood frog, Rana sylvatica. J. Exp. Zool. A Ecol. Genet. Physiol. 315: 487 - 494. 
Rubinsky B, Wong STS, Hong JS, Gilbert J, Roos M, Storey KB. 1994. ${ }^{1} \mathrm{H}$ magnetic resonance imaging of freezing and thawing in freeze-tolerant frogs. Am. J. Physiol. 266: 1771 - 1777.

Rubinsky B, Lee CY, Bastacky J, Onik J. 1987. The process of freezing and the mechanism of damage during hepatic cryosurgery. Cryobiology. 27: 85 - 97.

Rubinsky B, Wong STS, Hong JS, Gilbert J, Roos M, Storey KB. 1994. ${ }^{1} \mathrm{H}$ magnetic resonance imaging of freezing and thawing in freeze-tolerant frogs. Am. J. Physiol. 266: 1771 - 1777.

Russell EL, Storey KB. 1995. Glycogen synthetase and the control of cryoprotectant clearance after thawing in the freeze tolerant wood frog. Cryo-Lett. 16: 263-266.

Russo AA, effrey PD, Patten AK, Massagu , Pavletich NP. 1996. Crystal structure of the p27Kip1 cyclin-dependent- kinase inhibitor bound to the cyclin A-Cdk2 complex. Nature 382: $325-331$.

Ruuge EK, Ledenev AN, Lakomkin VL, Konstantinov AA, Ksenzenko MYu. 1991. Free radical metabolites in myocardium during ischemia and reperfusion. Am. J. Physiol. 261:81 - 86 .

Salazar M, Rojo AI, Velasco D, de Sagarra RM, Cuadrado A. 2006. Glycogen synthase kinase-3beta inhibits the xenobiotic and antioxidant cell response by direct phosphorylation and nuclear exclusion of the transcription factor Nrf2. J. Biol. Chem. 281: $14841-14851$.

Sander M, Griffen SC, Huang J, German MS. 1998. A novel glucose-responsive element in the human insulin gene functions uniquely in primary cultured islets. Proc. Natl. Acad. Sci. USA 95: 11572 - 11577.

Sarbassov DD, Guertin DA, Ali SM, Sabatini DM. 2005. Phosphorylation and regulation of Akt/PKB by the rictor-mTOR complex. Science 307: 1098 - 1101.

Schalm SS, Fingar DC, Sabatini DM, Blenis J. 2003. TOS motif-mediated raptor binding regulates 4E-BP1 multisite phosphorylation and function. Curr. Biol. 13: 797 806.

Sekulić A, Hudson CC, Homme L, Yin P, Otterness DM, Karnitz LM, Abraham RT. 2000. A direct linkage between the phosphoinositide 3-kinase-AKT signaling pathway and the mammalian target of rapamycin in mitogen-stimulated and transformed cells. Cancer Res. 60: $3504-3513$.

Sen P, Mukherjee S., Ray D, Raha S. 2003. Involvement of the Akt/PKB signaling pathway with disease processes. Mol. Cell. Biochem. 253: 241-246.

Shahbazian D, Roux PP, Mieulet V, Cohen MS, Raught B, Taunton J, Hershey JW, Blenis J, Pende M, Sonenberg N. 2006. The mTOR/PI3K and MAPK pathways converge on eIF4B to control its phosphorylation and activity. EMBO. J. 25: 2781 $-2791$.

Sherr CJ, Roberts JM. 1999. Cdk inhibitors: positive and negative regulators of G1-phase progression. Genes. Dev. 13: $1501-1512$. 
Sinclair BJ, Addo-Bediako A, Chown SL. 2003. Climatic variability and the evolution of insect freeze tolerance. Biol. Rev. Camb. Philos. Soc. 78: 181-195.

Sinclair BJ, Stinziano JR, Williams CM, Macmillan HA, Marshall KE, Storey KB. 2013. Real-time measurement of metabolic rate during freezing and thawing of the wood frog, Rana sylvatica: implications for overwinter energy use. J. Exp. Biol. 216: $292-302$.

Soos TJ, Kiyokawa H, Yan JS, Rubin MS, Giordano A, DeBlasio A, Bottega S, Wong B, Mendelsohn J, Koff A. 1996. Formation of p27-Cdk complexes during the human mitotic cell cycle. Cell Growth Differ. 7: $135-146$.

Storey JM, Storey KB. 1985. Triggering of cryoprotectant synthesis by the initiation of ice nucleation in the freeze-tolerant frog, Rana sylvatica. J. Comp. Physiol. B 156: 191-195.

Storey M, Storey KB. 1996. $\beta$-Adrenergic, hormonal and nervous influences on cryoprotectant. synthesis by liver of the freeze-tolerant wood frog Rana sylvatica. Cryobiology 33: $186-195$.

Storey KB. 1987a. Organ-specific metabolism during freezing and thawing in a freeze tolerant frog. Am. J. Physiol. 253: R292 - R297.

Storey KB. 1987b. Glycolysis and the regulation of cryoprotectant synthesis in liver of the freeze-tolerant wood frog. J. Comp. Physiol. B, 157: 373-380.

Storey KB. 2004a. Strategies for exploration of freeze responsive gene expression: advances in vertebrate freeze tolerance. Cryobiology 48: 134-145.

Storey KB. 2004b. Vertebrate freeze tolerance: role of freeze-responsive gene expression. In: Life in the Cold: Evolution, Mechanisms, Adaptation, and Application. 12th International Hibernation Symposium. (Barnes, B.M. and Carey, H.V., eds.) Biological Papers of the University of Alaska, \#27, Fairbanks. pp. 299-306.

Storey KB. 2006. Reptile freeze tolerance: metabolism and gene expression. Cryobiology 52: $1-16$.

Storey KB, Bischof J, Rubinsky B. 1992. Cryomicroscopic analysis of freezing in liver of the freeze-tolerant wood frog. Am. J. Physiol. 263: 185 - 194.

Storey KB, Mommsen TP. 1994. Effects of temperature and freezing on hepatocytes isolated from a freeze tolerant frog. Am. J. Physiol. 266: R1477 - R1482.

Storey KB, Storey JM. 1984. Biochemical adaptation for freezing tolerance in the wood frog, Rana sylvatica. J. Comp. Physiol. B 155: 29-36.

Storey KB, Storey JM. 1989. Freeze tolerance and freeze avoidance in ectotherms. in Wang, L.C.H. (ed) Animal Adaptation to Cold, Heidelberg: Springer-Verlag, pp. $51-82$.

Storey KB. 1990. Life in a frozen state: adaptive strategies for natural freeze tolerance in amphibians and reptiles. Am. J. Physiol. 258: R559 - R568.

Storey KB. 2004a. Functional Metabolism Regulation and Adaptation, Published by John Wiley \& Sons, Inc., Hoboken, New Jersey. 
Storey KB. 2004b. Vertebrate freeze tolerance: role of freeze-responsive gene expression. In: Life in the Cold: Evolution, Mechanisms, Adaptation, and Application. 12th International Hibernation Symposium. (Barnes, B.M. and Carey, H.V., eds.) Biological Papers of the University of Alaska, \#27, Fairbanks. pp. 299-306.

Storey KB. 2004c. Strategies for exploration of freeze responsive gene expression: advances in vertebrate freeze tolerance. Cryobiology 48:134-145.

Storey KB, Storey JM. 1984. Biochemical adaptation for freezing tolerance in the wood frog, Rana sylvatica. J Comp. Physiol. B. 155: 29-36.

Storey KB, Storey JM. 1986a. Freeze tolerance and intolerance as strategies of winter survival in terrestrially-hibernating amphibians. Comp. Biochem. Physiol. A, 83: 613-617.

Storey KB, Storey JM. 1986b. Freeze-tolerant frogs: cryoprotectants and tissue metabolism during freeze/thaw cycles. Can. J. Zool. 64: 49-56.

Storey KB, Storey JM. 1988. Freeze tolerance in animals. Physiol. Rev 68: 27-84.

Storey KB, Storey JM. 2001. Signal transduction and gene expression in the regulation of natural freezing survival. in Storey KB, Storey JM (eds) Cell and Molecular Responses to Stress, Amsterdam: Elsevier Press, Vol. 2, pp. 1-19.

Storey KB, Storey JM. 2004a. Physiology, biochemistry and molecular biology of vertebrate freeze tolerance: the wood frog. In: Life in the Frozen State (Benson, E., Fuller, B., and Lane, N., eds.) CRC Press, Boca Raton, pp. 243-274.

Storey KB, Storey JM. 2004b. Metabolic rate depression in animals: transcriptional and translational controls. Biol. Rev. Camb. Philos. Soc. 79(1): 207-233.

Storey KB, Storey JM. 2011. Heat shock proteins and hypometabolism: adaptive strategy for proteome preservation. Res. Rep. Biol. 2: 57-68.

Storey KB, Storey JM. 2012. Insect cold hardiness: recent advances in metabolic, gene and protein adaptation. Can. J. Zool. 90: 456-475.

Suzuki A, Tsutomi Y, Yamamoto N, Shibutani T, Akahane K. 1999. Mitochondrial regulation of cell death: mitochondria are essential for procaspase 3-p21 complex formation to resist Fas-mediated cell death. Mol. Cell. Biol. 19: 3842 - 3847.

Syed NA, Khandelwal RL. 2000. Reciprocal regulation of glycogen phosphorylase and glycogen synthase by insulin involving phosphatidylinositol-3 kinase and protein phosphatase-1 in HepG2 cells. Mol Cell Biochem. 211: 123 - 136.

Traystman RJ, Kirsch JR, Koehler RC. 1991. Oxygen radical mechanisms of brain injury following ischemia and reperfusion. J. Appl. Physiol. 71: 1185 - 1195.

Valcourt JR, Lemons JM, Haley EM, Kojima M, Demuren OO, Coller HA. 2012. Staying alive: metabolic adaptations to quiescence. Cell Cycle 11: 1680 - 1696.

Van Dam EM, Govers R, James DE. 2005. Akt activation is required at a late stage of insulin-induced GLUT4 translocation to the plasma membrane. Mol Endocrinol. 19:1067 - 1077 . 
Vander Haar E, Lee SI, Bandhakavi S, Griffin TJ, Kim DH. 2007. Insulin signalling to mTOR mediated by the Akt/PKB substrate PRAS40. Nat. Cell. Biol. 9: 316 - 323.

Waga S, Hannon GJ, Beach D, Stillman B. 1994. The p21 inhibitor of cyclin-dependent kinases controls DNA replication by interaction with PCNA. Nature 369: 574 578.

Wang L, Harris TE, Roth RA, Lawrence JC Jr. 2007. PRAS40 regulates mTORC1 kinase activity by functioning as a direct inhibitor of substrate binding. J. Biol. Chem. 282: $20036-20044$.

Wang L, Lawrence JC Jr, Sturgill TW, Harris TE. 2009. Mammalian target of rapamycin complex 1 (mTORC1) activity is associated with phosphorylation of raptor by mTOR. J. Biol. Chem. 284: 14693 - 14697.

Whiteman EL, Cho H, Birnbaum MJ. 2002. Role of Akt/protein kinase B in metabolism. Trends Endocrinol. Metab. 13: $444-451$.

Xu N, Hegarat N, Black EJ, Scott MT, Hochegger H, Gillespie DA. 2010. Akt/PKB suppresses DNA damage processing and checkpoint activation in late G2. J. Cell Biol. 190: 297 - 305 .

Yamashita H, Takenoshita M, Sakurai M, Bruick RK, Henzel WJ, Shillinglaw W, Arnot D, Uyeda K. 2001. A glucose-responsive transcription factor that regulates carbohydrate metabolism in the liver. Proc. Natl. Acad. Sci. USA 98: 9116 9121.

Yang J, Cron P, Thompson V, Good VM, Hess D, Hemmings BA, Barford D. 2002. Molecular mechanism for the regulation of protein kinase B/Akt by hydrophobic motif phosphorylation. Mol. Cell. 9: 1227 - 1240.

Yang R, M 1 ler C, Huynh , F ung YK, Yee AS, Koeffler HP. 1999. Functions of cyclin $\mathrm{A} 1$ in the cell cycle and its interactions with transcription factor E2F-1 and the Rb family of proteins. Mol. Cell. Biol. 19: 2400 - 2407.

Yiangou M, Tsapogas P, Nikolaidis N, Scouras ZG. 1997. Heat shock gene expression during recovery after transient cold shock in Drosophila auraria (Diptera: Drosophilidae). Cytobios. 92: 91-98.

Yuan H, Kamata M, Xie YM, Chen IS. 2004. Increased levels of Wee-1 kinase in G(2) are necessary for Vpr-and gamma irradiation-induced G(2) arrest. J. Virol. 78: $8183-8190$.

Zhang DD. 2006. Mechanistic studies of the Nrf2-Keap1 signaling pathway. Drug Metab. Rev. 38: 769 - 789.

Zhang J, Tessier SN, Storey KB. 2011. PI3K-Akt regulation as a molecular mechanism of the stress response during aerobic dormancy. In: Nowakowska, A. and Caputa, M.,(Eds.), Hypometabolism: Strategies of Survival in Vertebrates and Invertebrates. Research Signpost, Kerala, India, pp. 147-182.

Zhang J, Biggar KK, Storey KB. 2013. Regulation of p53 by reversible posttranscriptional and post-translational mechanisms in liver and skeletal muscle of an anoxia tolerant turtle, Trachemys scripta elegans. Gene. 513: $147-155$. 
Zhang S, Cai G, Fu B, Feng Z, Ding R, Bai X, Liu W, Zhuo L, Sun L, Liu F, Chen X. 2012. SIRT1 is required for the effects of rapamycin on high glucose-inducing mesangial cells senescence. Mech. Ageing Dev. 133: 387 - 400 .

Zhou BP, Liao Y, Xia W, Spohn B, Lee MH, Hung MC. 2001. Cytoplasmic localization of p21Cip1/WAF1 by Akt-induced phosphorylation in HER-2/neu-overexpressing cells. Nat. Cell Biol. 3: 245 - 252.

Zhao X, Gan L, Pan H, Kan D, Majeski M, Adam SA, Unterman TG. 2004. Multiple elements regulate nuclear/cytoplasmic shuttling of FOXO1: characterization of phosphorylation- and 14-3-3-dependent and -independent mechanisms. Biochem. J. 378: $839-849$.

Zhao X, Sun G, Zhang J, Strong R, Dash PK, Kan YW, Grotta JC, Aronowski J. 2007. Transcription factor Nrf2 protects the brain from damage produced by intracerebral haemorrhage. Stroke 38: 3280 - 3286.

Zhu H, Santo A, Li Y. 2012. The antioxidant enzyme peroxiredoxin and its protective role in neurological disorders. Exp. Biol. Med. (Maywood). 237:143 - 149. 


\section{Appendix A: Explaination of immublotting}

Fig. A1

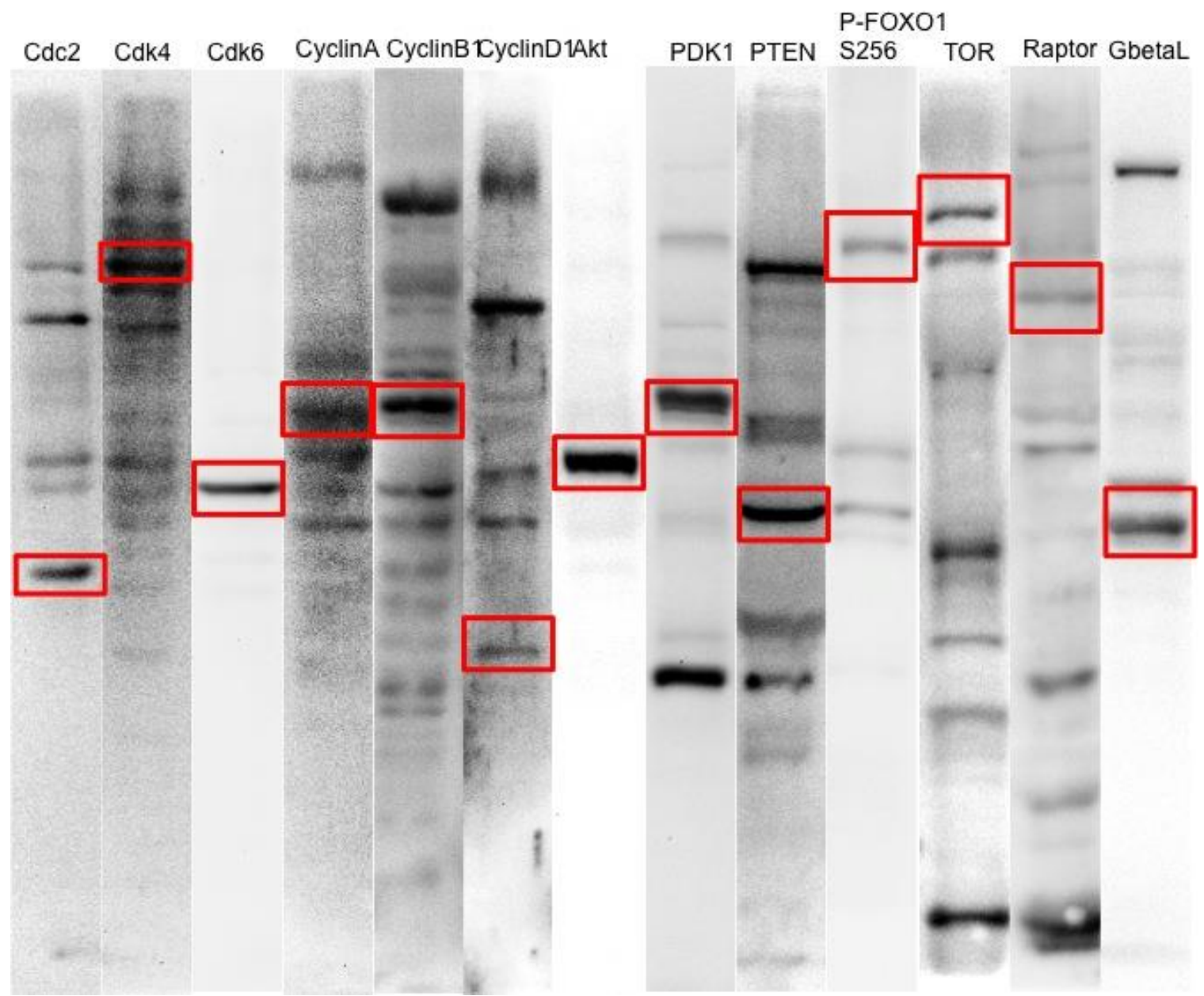

Fig. A1. Example immunoblotting bands for proteins of interest (highlighted in box). All bands were chosen at the correct molecular weight and using squirrel as a positive reference. All antibodies were checked for source and epitope from suppliers. If there were multiple bands around the protein band of interest, the following test could be done: two antibodies recognizing the same protein with different epitopes can be used in a WBIP experiment with one antibody as a pull down antibody and the other for immunoblotting, thereby the protein band will be verified by two different antibodies. 


\section{Appendix B: Communications at scientific meetings}

$\underline{2013}$

Zhang, J. and Storey, K.B. DNA methylation and regulation of DNMTs in freeze tolerant wood frog, Rana sylvatica. $10^{\text {th }}$ Annual Ottawa-Carleton Institute of Biology Symposium, Carleton University, Ottawa, ON, 2013

$\underline{2012}$

Zhang, J. and K.B. Storey. p53 transcription factor and cell cycle arrest during anoxia in turtles, Trachemys scripta elegans. $1^{\text {st }}$ International Conference on Oxidative Stress in Aquatic Ecosystems. Los Cabos, Mexico, November 20-24, 2012.

Zhang, J. and Storey, K.B. Regulation of mTOR complex 1 in liver of freeze tolerant wood frog, Rana sylvatica. $19^{\text {th }}$ Methods in Protein Structure Analysis conference and $2^{\text {nd }}$ China-Canada Symposium for Systems Biology Conference, Ottawa, June 25-28, 2012

Zhang, J. and Storey, K.B. mTOR Regulation of mTOR complex 1 in liver of freeze tolerant wood frog, Rana sylvatica. $9^{\text {th }}$ Annual Ottawa-Carleton Institute of Biology Symposium, Carleton University, Ottawa, ON, 2012

Zhang, J. and Storey, K.B. Involvement of microRNA in the activation of Akt/PKB signaling in wood frog liver during freezing stress. Society for Cryobiology, $49^{\text {th }}$ Annual Meeting, Rosario, Argentina, June 3-6, 2012.

$\underline{2011}$

Zhang, J. and Storey, K.B. Tissue-specific pattern of PI3K-Akt signaling during freezing in wood frog, Rana sylvatica. 26th Annual meeting, Federação de Sociedades de Biologia Experimental (FeSBE), Rio de Janeiro, Brazil, August 24-27, 2011.

Zhang, J. and Storey, K.B. Involvement of microRNA in the activation of Akt/PKB signaling in wood frog liver during freezing. 26th Annual meeting, Federação de Sociedades de Biologia Experimental, Rio de Janeiro, Brazil, August 24-27, 2011.

Zhang, J. and Storey, K.B. p53 transcription factor and cell cycle arrest during anoxia in turtles, Trachemys scripta elegans. 26th Annual meeting, Federação de Sociedades de Biologia Experimental, Rio de Janeiro, Brazil, August 24-27, 2011.

(Oral) Zhang, J. and Storey, K.B. Involvement of microRNA in the activation of Akt/PKB signaling in wood frog liver during freezing stress. $50^{\text {th }}$ Canadian Society of Zoology Conference, University of Ottawa, Ottawa, ON, May 16-20, 2011

Zhang, J. and Storey, K.B. Involvement of microRNA in the activation of Akt/PKB signaling in wood frog liver during freezing stress. $8^{\text {th }}$ Annual Ottawa-Carleton Institute of Biology Symposium, Carleton University, Ottawa, ON, 2011 
$\underline{2010}$

Zhang, J. and Storey, K.B. Tissue-specific pattern of PI3K-Akt signaling during freezing in wood frogs, Rana sylvatica. $5^{\text {th }}$ annual Canadian Society for Life Science Research Conference, McGill University, Montreal, PQ, August 13-14, 2010

Zhang, J. and Storey, K.B. Tissue-specific pattern of PI3K-Akt signaling during freezing in wood frogs, Rana Sylvatica. $7^{\text {th }}$ annual Ottawa-Carleton Institute of Biology Symposium, University of Ottawa, Ottawa, ON. 2010

$\underline{2009}$

Zhang, J. and Storey, K.B. p53 Transcription factor and cell cycle arrest during anoxia in turtles, Trachemys scripta elegans. $12^{\text {th }}$ Chemistry and Biochemistry Graduate Research Conference, Concordia University, Montreal, PQ, November 20-21, 2009

Zhang, J. and Storey, K.B. p53 Transcription factor and cell cycle arrest during anoxia in turtles, Trachemys scripta elegans. $48^{\text {th }}$ Canadian Society of Zoology Conference, University of Toronto, Toronto, ON, May 12-16, 2009

Zhang, J and Storey, K.B. p53 Transcription factor and cell cycle arrest during anoxia in turtles, Trachemys scripta elegans. $6^{\text {th }}$ annual Ottawa-Carleton Institute of Biology Symposium, Carleton University, Ottawa, ON. 2009 